Uili norden

PPCP monitoring in the Nordic Countries

- Status Report

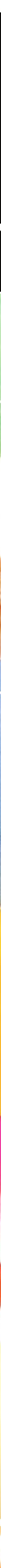



4 norden 



\section{PPCP monitoring in the Nordic Countries - Status Report}


PPCP monitoring in the Nordic Countries - Status Report

TemaNord 2012:519

ISBN 978-92-893-2346-8

http://dx.doi.org/10.6027/TN2012-519

(C) Nordic Council of Ministers

Print: Kailow Express ApS

Cover photo: Karin Beate Nøsterud, ImageSelect

This publication has been published with financial support by the Nordic Council of Ministers. However, the contents of this publication do not necessarily reflect the views, policies or recommendations of the Nordic Council of Ministers.

www.norden.org/en/publications

\section{Nordic co-operation}

Nordic co-operation is one of the world's most extensive forms of regional collaboration, involving Denmark, Finland, Iceland, Norway, Sweden, and the Faroe Islands, Greenland, and Åland.

Nordic co-operation has firm traditions in politics, the economy, and culture. It plays an important role in European and international collaboration, and aims at creating a strong Nordic community in a strong Europe.

Nordic co-operation seeks to safeguard Nordic and regional interests and principles in the global community. Common Nordic values help the region solidify its position as one of the world's most innovative and competitive.

\section{Nordic Council of Ministers}

Ved Stranden 18

1061 København $\mathrm{K}$

Telefon (+45) 33960200

www.norden.org 


\section{Content}

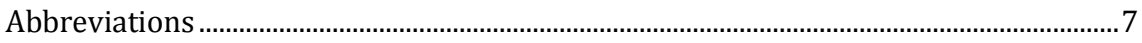

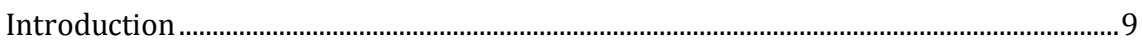

1. Methodology ....................................................................................................... 11

2. Analgesics and anti-inflammatories.................................................................... 17

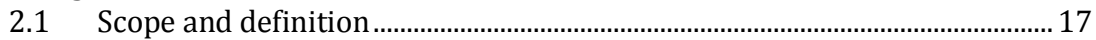

2.2 Compounds mapped............................................................................................ 18

2.3 Compounds monitored in at least two studies ................................................ 46

$2.4 \quad$ Compounds monitored in one study ……....................................................... 47

3. Antibiotics, antiseptics, germicides and anti-fungals ............................................... 49

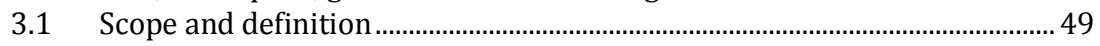

3.2 Compounds mapped.................................................................................... 51

3.3 Compounds monitored in at least two studies ................................................104

3.4 Compounds monitored in one study ...........................................................117

4. Hormones and hormone antagonists .................................................................... 127

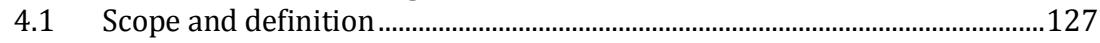

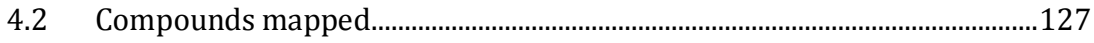

4.3 Compounds monitored in one study ………................................................152

5. Cardiac and blood pressure medicine and diuretics..................................................155

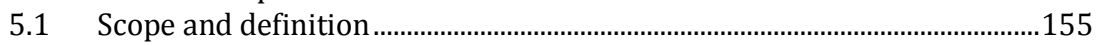

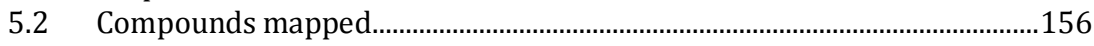

5.3 Compounds monitored in at least two studies ...............................................185

5.4 Compounds monitored in one study ..............................................................188

6. Complexing compounds....................................................................................... 191

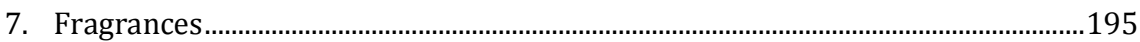

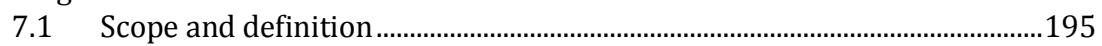

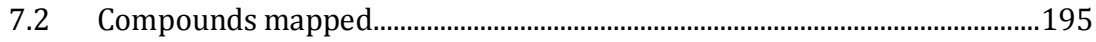

7.3 Compounds monitored in one study ...........................................................220

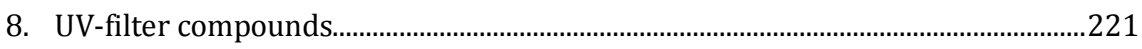

8.1 Scope and definition..................................................................................22

8.2 Compounds monitored in at least two studies .............................................222

8.3 Compounds monitored in one study …….....................................................222

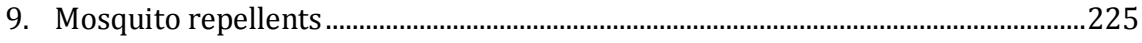

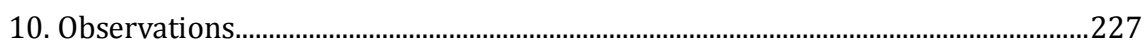

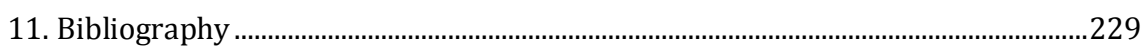





\section{Abbreviations}

$\mathrm{AD}$

CAS nr

$\mathrm{dw}$

EC50

fw

Hospital WW

LC50

LD50

LOEC

Max

Min

NA

ND

NOEC

Nr.S

PNEC

PPCP

Ref

STP

WW average detected value, this average does not include non detected values

chemical abstract service number

dry weight

effect concentration $50 \%$

fat weight

hospital waste water

lethal concentration $50 \%$

lethal dose $50 \%$

lowest observed effect concentration

maximum

minimum

not available

not detected

no observed effect concentration

number of samples

predicted non-effect concentration

pharmaceuticals and personal care products

reference

sewage treatment plant

wet weight 



\section{Introduction}

Pharmaceuticals and Personal Care Products (PPCPs) are substances used by individuals for personal health or cosmetic reasons, and products used by agribusiness to boost growth or health of livestock. Personal care products, such as creams, detergents, deodorants, cosmetics, perfumes etc. are used in an increasing extent worldwide. These products contain a wide variety of chemicals, used either as an active ingredient or as an additive to improve qualities of the product.

Chemicals used in PPCPs are expected to emit into the environment in their consumed form or in a degraded form. The question remains as regards to which compounds are present in our environment, and if they may negatively impact the environment and public health. In this manner, in the past few years, there has been an increased focus on the environmental impacts of pharmaceuticals, both human and veterinary, and personal care products. Thereby, environmental monitoring has been carried out to assess the occurrence of PPCPs in different compartments of the environment.

Several PPCP monitoring reports have been published in the Nordic countries. However, to date, no general overview of the results in the Nordic countries has been established.

The objective of this report is to give an overview of the results of PPCP monitoring in the Nordic countries, by identifying which compounds have been monitored, in which countries and if the are important differences in the values detected amongst the Nordic countries. This report presents the results and does not intend to interpret the results.

This project has been initiated by the Nordic Screening group and financed by the Nordic Council of Ministers.

The monitoring data available is illustrated as maps, graphs and tables, which will allow the reader to rapidly identify the countries in which compounds have been monitored and appreciate the average values detected in the different countries as well as the disparity of the results within a country and amongst the Nordic countries. 



\section{Methodology}

The objective of this report is to give an overview of the existing monitoring data available for PPCPs in the Nordic environment. This report focuses on making extensive monitoring data easily available to the reader and does not focus on giving an interpretation to the results detected.

PPCP includes a very extensive number of compounds. The Nordic screening group selected the following compound groups to be included in this report:

- Analgesics and anti-inflammatories

- Antibiotics, antiseptics and germicides, anti-fungals

- Hormones (synthetic and natural) and hormone antagonists

- Cardiac, blood pressure medicine and diuretics

- Complexing forms

- Fragrances

- UV-filter compounds

- Mosquito repellents

In order to have an overview of the various PPCP monitoring data in the Nordic countries, each Nordic country identified relevant monitoring reports. All the reports were reviewed and compounds belonging to the PPCP groups of interest were identified. Metabolites were not included in this report.

The large majority of PPCPs for human use are expected to emit to the environment via the waste water system. Indeed most pharmaceuticals are evacuated by the urines and faeces. In the same way personal care products, which will be used directly on skin and hair are expected to be washed off and end in the waste water system.

Pharmaceuticals for veterinary use are expected to be found in soil and leachates of lands used for animal breeding or water and sediment surrounding fish farms.

The monitoring reports reviewed included monitoring in the following matrices:

- Sewage treatment plants (STPs): influent, effluent and sludge

- Surface water, included water and sediment samples: STP recipient, up and down stream from fish farms, other fresh or sea waters

- Groundwater, in particular at proximity of hospitals and farms 
- Air samples

- Hospitals waste water eachates/runoff from landfills

- Farms: soil, manure, runoff

Some of the reports included monitoring data from background locations (reference values), in particular for surface water, sediment and air.

In a few studies, monitoring was also carried out at different process/treatment stages of STPs and various industrial plants. The results from the latter monitoring are not presented in this report, as they only reflect the removal rate at different stages of the process.

Only monitoring data was considered of interest for this report. Predicted environmental concentrations were not considered. In the same way, averages quoted in studies with no reference to sampling locations and results were not used, with the exception of one Finnish study (Vieno, 2008), as there was little data available for this country.

\section{Compounds monitored in a matrix in three or more studies}

Compounds monitored in three or more studies in the same matrix are presented in this report as maps and graphs. Compounds monitored in two or more studies in biota are also presented as maps and graphs. However, influent levels were not considered for mapping and graphs as the removal rate will vary depending on the STP processes used and will thereby not be relevant to appreciate the potential levels found in the environment. Hospital effluent is expected be transferred to the local waste water treatment plant and consequently contributes to STP influent. In this manner hospital effluent was not illustrated in maps and graphs either.

As indicated in the example below, the map indicates the sampling location and matrices monitored. Each country has been attributed a colour which is also referred to in the graphs and tables, and each matrix is represented by a different symbol. The objective of the maps is to allow the ready to rapidly have an overview of where the compound has been monitored and appreciate the scope of the monitoring in each country. The monitoring locations indicated on the maps represent the city in which the sampling was carried out and not the precise GPS location of of the sample.

For each matrix monitored three or more times, a graph is available to present the results for each location monitored. Graphs are also available for compounds monitored in biota in two or more studies. In the case where a same sampling location was monitored more than once, only one result will be presented for this location and it will correspond to the average detected value for this specific location, this average does not take into consideration non-detected values. Background values were not differenciated from the rest of the values for the same matrix. 

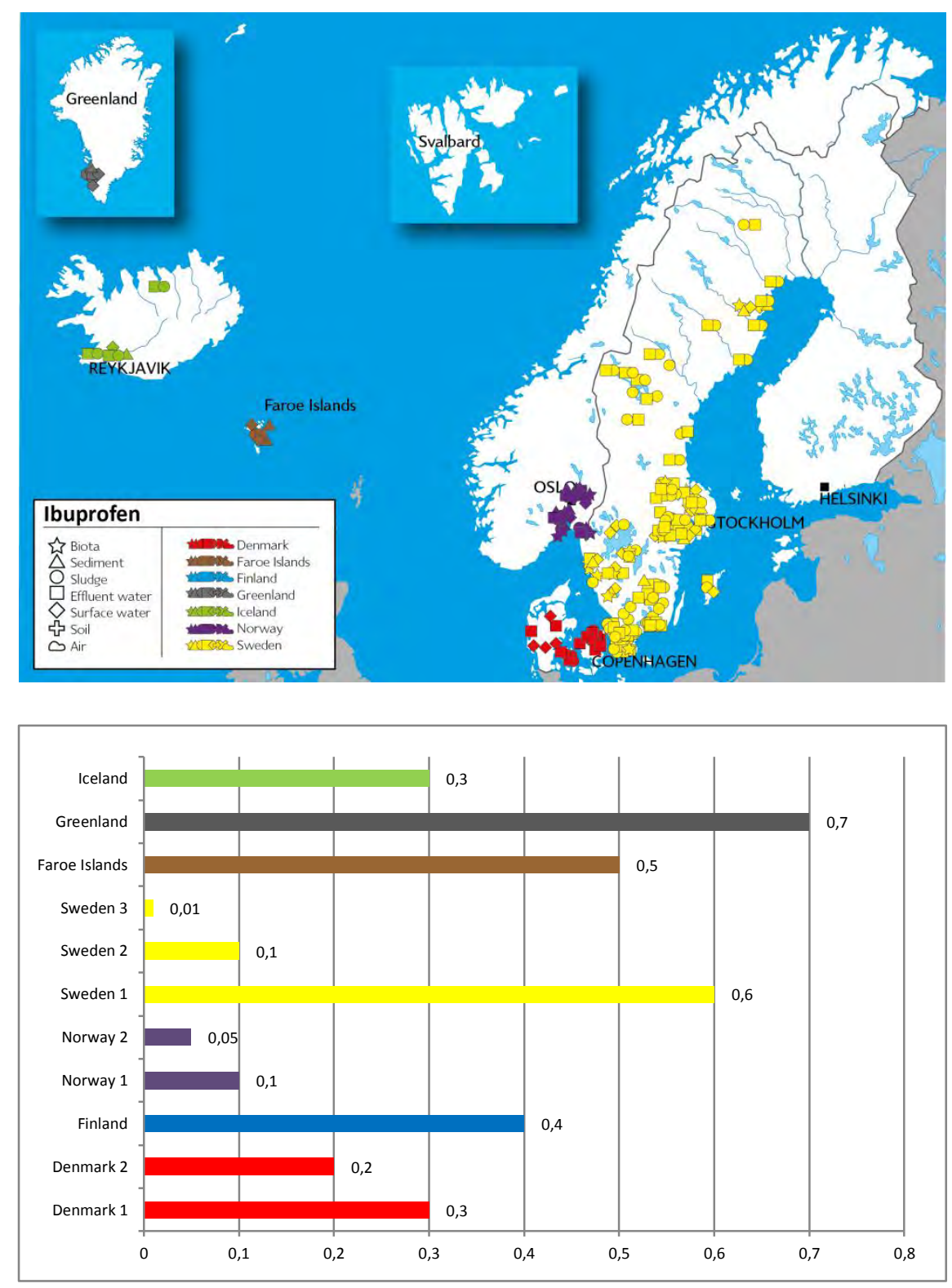

In the case, a compound was monitored in the same matrix in more than two studies but was not detected in any samples; the results were not presented as a graph.

\section{Compounds monitored in at least two studies}

All compounds monitored in at least two studies are presented in tables as that below. The tables present the minimum and maximum values detected and the average detected value (AD) for each matrix, per country. The average detected value (AD) does not take into consideration non-detected values. The total number of samples (NS) analysed for one given matrix and country is indicated. The latter will allow the reader to appreciate the representivity of the results. 
The results for all matrices monitored for the compound are indicated in the table, including STP influent and hospital waste water values.

\begin{tabular}{|c|c|c|c|c|c|c|c|c|}
\hline & \multicolumn{2}{|c|}{ Denmark } & \multicolumn{2}{|c|}{ Finland } & \multicolumn{2}{|c|}{ Sweden } & \multicolumn{2}{|c|}{ Iceland } \\
\hline Matrix & $\begin{array}{r}\min \\
\max \\
A D\end{array}$ & $\begin{array}{l}\text { Nr.S } \\
\text { (ref) }\end{array}$ & $\begin{array}{r}\min \\
\max \\
A D\end{array}$ & $\begin{array}{l}\text { Nr.S } \\
\text { (ref) }\end{array}$ & $\begin{array}{r}\min \\
\max \\
A D\end{array}$ & $\begin{array}{l}\text { Nr.S } \\
\text { (ref) }\end{array}$ & $\begin{array}{r}\min \\
\max \\
A D\end{array}$ & $\begin{array}{l}\text { Nr.S } \\
\text { (ref) }\end{array}$ \\
\hline Matrix 1 & nd & 6 (a) & nd & $3(c)$ & $\begin{array}{l}0,01 \\
0,06 \\
0,02\end{array}$ & 16 (def) & nd & $2^{4}(g)^{d}$ \\
\hline Matrix 2 & $\begin{array}{l}0,1^{1} \\
0,3^{2} \\
0,2^{3}\end{array}$ & $17(a b)$ & $\begin{array}{l}\text { nd } \\
0,5 \\
0,3\end{array}$ & $3(c)$ & $\begin{array}{l}\text { nd } \\
0,9 \\
0,3\end{array}$ & 64(def) & $\begin{array}{r}0,01 \\
0,6 \\
0,2\end{array}$ & $6^{5}(\mathrm{~g})$ \\
\hline Matrix 3 & nd & 10(a) & nd & 2(c) & nd & 9(df) & nd & $5(\mathrm{~g})$ \\
\hline
\end{tabular}

1: Minimum

2: Maximu

3: Average detected

4: References

5: Number of samples

\section{References:}

a) (Aaa et al, 2001)

b) (Bbb et al, 2002)

c) (Ccc et al, 2003)

d) (Ddd et al, 2004)

e) (Eee et al, 2005)

f) (Fff et al, 2001)

g) (Ggg et al, 2002)

When the compound was monitored in a matrix in more than one study for one country, the minimum, maximum and average detected value were determined from all the monitoring data available for that country and not specified for each study. In the same way, no difference was made between the values detected in "background" locations and other locations monitored. The tables express the minimum, maximum and average detected values for a country and a given matrix.

Available PNEC values are also indicated in order to allow the reader to easily appreciate the significance of the monitored values. Available toxicity data is also indicated.

The toxicity reference values and PNEC values indicated in this report were identified in monitoring studies assessed in this report.

A factual conclusion is given for each compound. The conclusion does not intend to give an interpretation to the results but an overview of the main observations. 


\section{Compounds monitored in only one study}

Compounds monitored in only one study are grouped by study and presented in tables which indicate the minimum and maximum values detected and the average detected value (AD) for each matrix. No PNEC or toxicity reference values are indicated for these compounds.

\section{Additional specifications}

On the contrary of the graphs, in tables, all results were considered individually regardless of whether the results were from a same monitoring location. In this manner, for some compounds the minimum and maximum values indicated in a graph may vary from the minimum and maximum values indicated in a table. As mentioned earlier, in the case where a same sampling location was monitored more than once, only one result will be presented for this location in a graph and it will correspond to the average detected value for this specific location.

In the case of the Finnish study (Vieno, 2008), as the monitoring locations were not specified, the study is illustrated as "14 STPs" on maps and in graphs. For most compounds monitored in this study, the results were presented as the "mean" value for all 14 STPs. In this manner in tables the minimum and maximum values are indicated as not available (NA) and the average detected value corresponds to the "mean" value indicated in the study. Whenever the maximum or minimum values detected were indicated in the study, the latter was indicated in the table. 



\section{Analgesics and anti-inflammatories}

\subsection{Scope and definition}

Analgesics are defined as drugs or medicine given to reduce pain without resulting in loss of consciousness. Analgesics are sometimes referred to as painkiller medications.

Anti-inflammatory are defined as medicine used to relieve swelling, pain, and other symptoms of inflammation. In this manner some antiinflammatories also have analgesic effects and thereby are included in this category.

Table 1 presents the compounds classified in this category and identified in monitoring studies in the Nordic countries.

\begin{tabular}{ll} 
Table 1: Analgesics and anti-inflammatories monitored in the Nordic countries \\
\hline Compounds monitored & CAS nr \\
\hline Acetylsalicylic acid (analgesic) & $50-78-2$ \\
Buprenorphine (analgesic) & $52485-79-7$ \\
Codeine (analgesic) & $76-57-3$ \\
Dextropropoxyphen (analgesic) & $469-62-5$ \\
Diclofenac (inflammatories) & $15307-86-5$ \\
Fentanyl (narcotic analgesic) & $437-38-7$ \\
lbuprofen (anti-inflammatory & $15687-27-1$ \\
Ketoprofen (anti-inflammatory) & $22071-15-4$ \\
Morphine (analgesic) & $57-27-2$ \\
Naproxen (anti-inflammatory) & $22204-53-1$ \\
Oxycondone (analgesic) & $76-42-6$ \\
Paracetamol (anti-inflammatory) & $103-90-2$ \\
Tramadol (analgesic) & $46941-76-8 / 27203-92-5$
\end{tabular}




\subsection{Compounds mapped}

\subsubsection{Acetylsalicylic Acid}

CAS nr.: 50-78-2

Info: Globally known as Aspirin, analgesic and anti-inflammatory. Toxicity data: NA

$P N E C=N A$

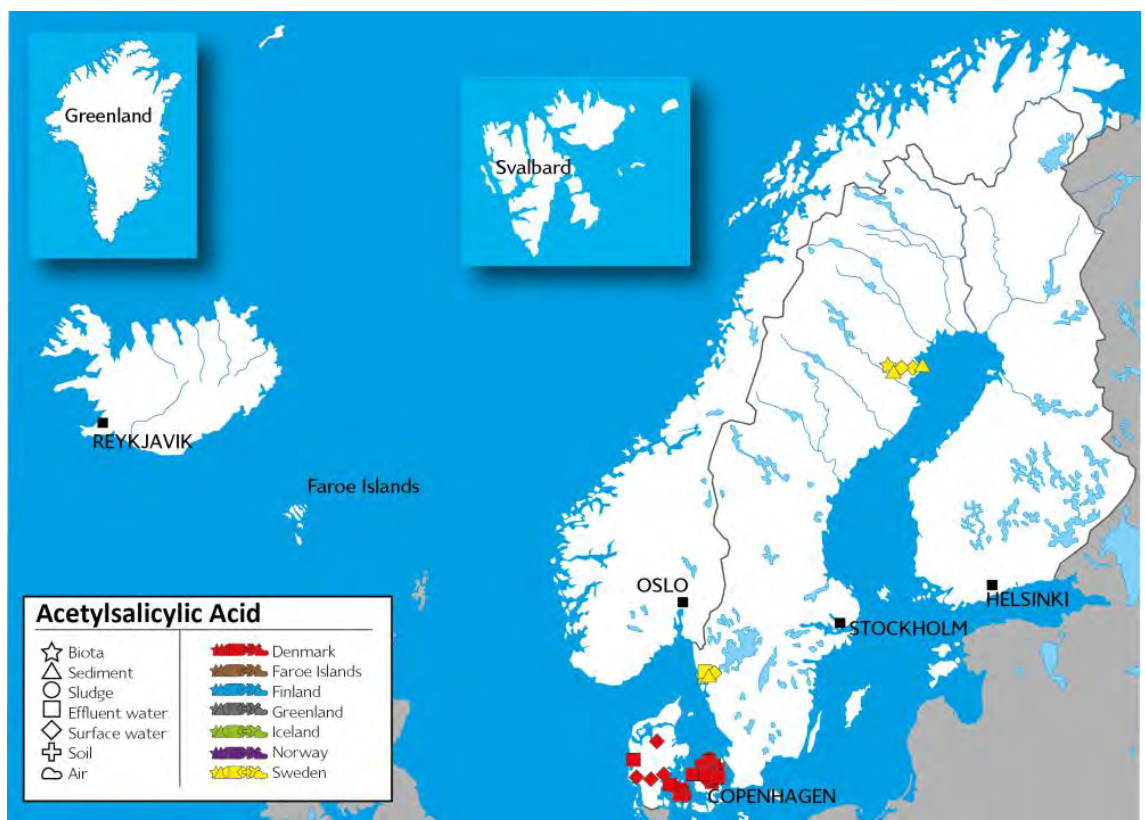

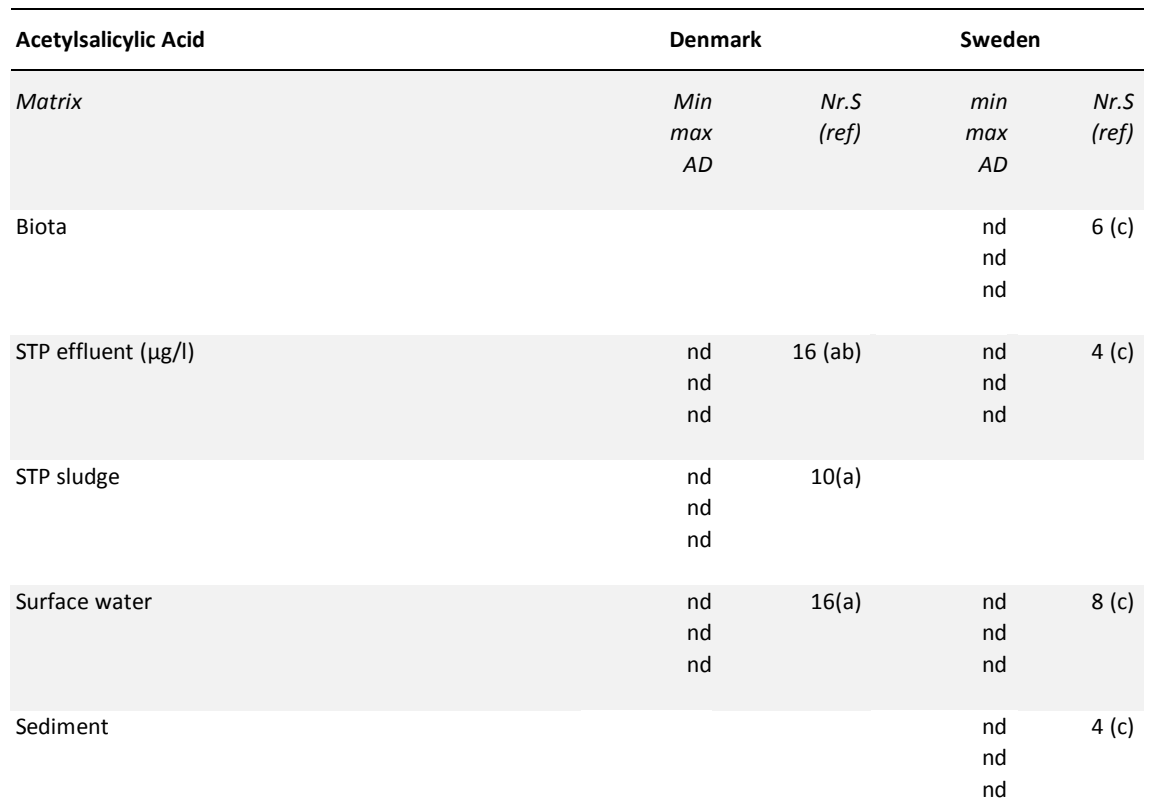




\begin{tabular}{|c|c|c|c|c|}
\hline $\begin{array}{l}\text { Acetylsalicylic Acid } \\
\text { STP Influent ( } \mu \mathrm{g} / \mathrm{l})\end{array}$ & \multicolumn{2}{|c|}{ Denmark } & \multicolumn{2}{|c|}{ Sweden } \\
\hline & $\begin{array}{r}\text { nd } \\
2 \\
1,6\end{array}$ & 9(a) & $\begin{array}{l}\text { nd } \\
\text { nd } \\
\text { nd }\end{array}$ & 4 (c) \\
\hline Warning system for pesticide leaching to groundwater & $\begin{array}{l}\text { nd } \\
\text { nd } \\
\text { nd }\end{array}$ & $7(\mathrm{a})$ & & \\
\hline Pig slurry & $\begin{array}{l}\text { nd } \\
\text { nd } \\
\text { nd }\end{array}$ & $4(\mathrm{a})$ & & \\
\hline Groundwater & $\begin{array}{l}\text { nd } \\
\text { nd } \\
\text { nd }\end{array}$ & $3(\mathrm{a})$ & & \\
\hline Drain water & $\begin{array}{l}\text { nd } \\
\text { nd } \\
\text { nd }\end{array}$ & $1(\mathrm{a})$ & & \\
\hline
\end{tabular}

\section{References:}

a) (Mogensen, et al., 2008)

b) (Kjølholt, et al., 2003)

c) (Remberger, et al., 2009)

\section{Conclusion:}

The compound has been monitored in two Danish studies and one Swedish study. It was only detected in STP influent samples in Denmark, not in Sweden. The compound was not detected in any other matrix. 


\subsubsection{Diclofenac}

CAS nr.: 15307-86-5

Info: Non-steroidal anti-inflammatory drug Toxicity data: NA

$P N E C=N A$
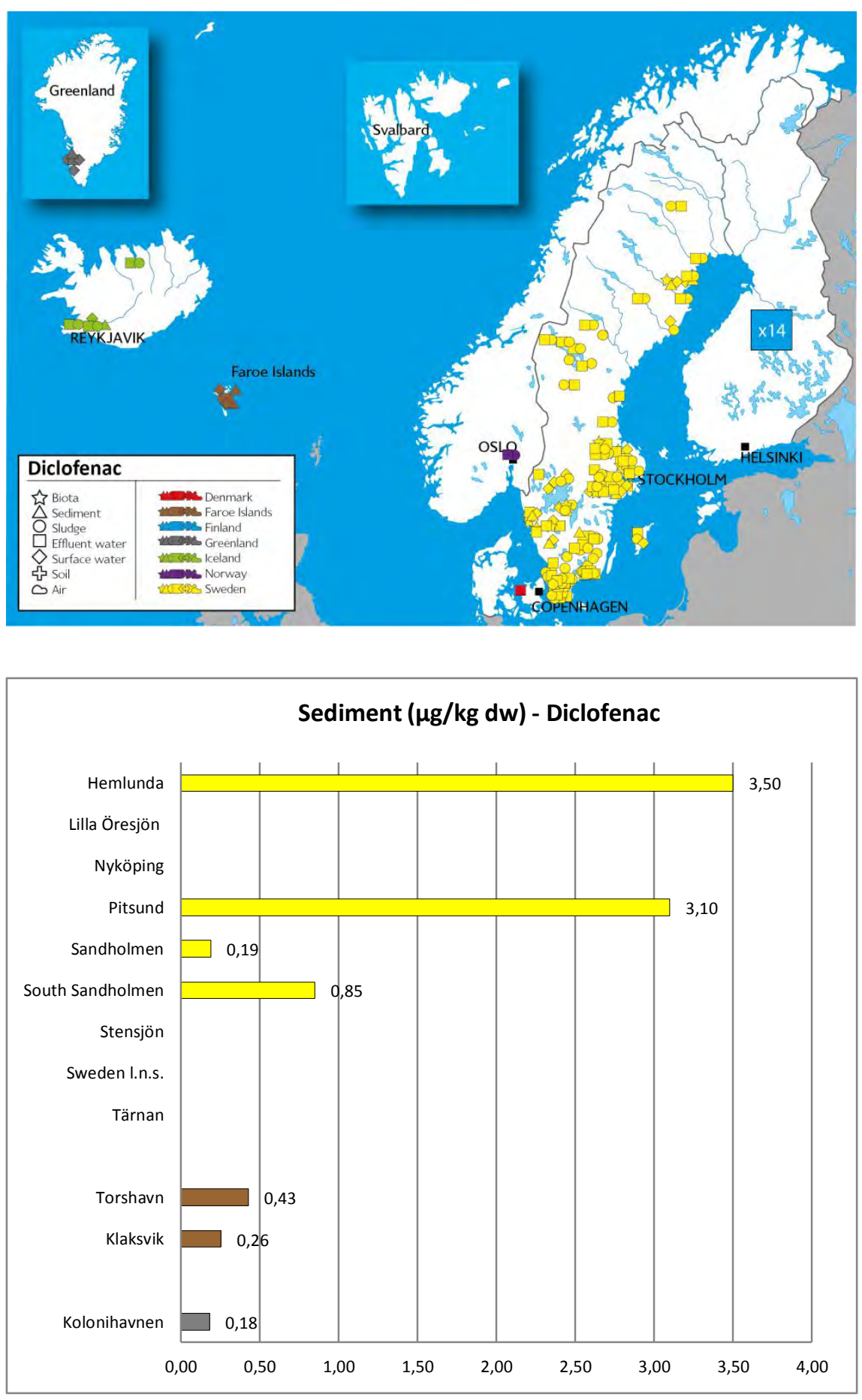


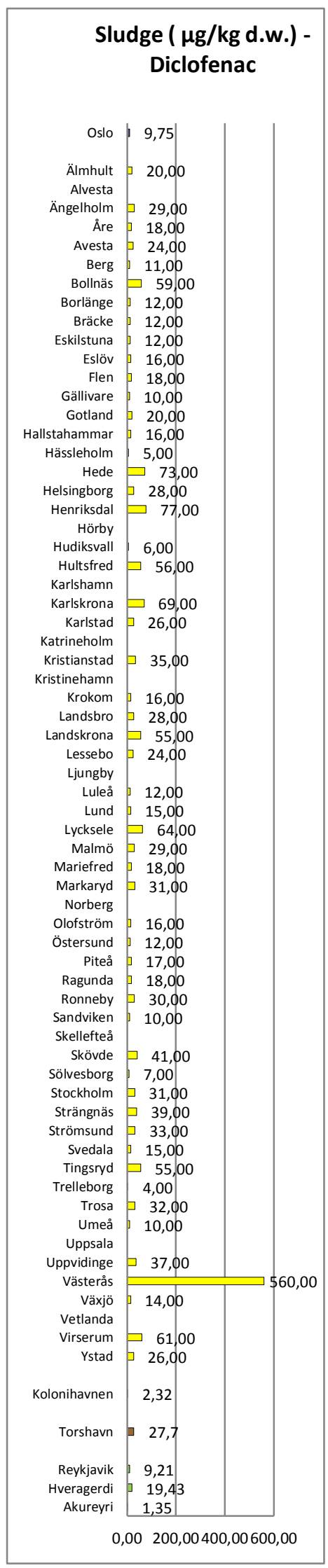

\section{STP Effluent Water $(\mu \mathrm{g} / \mathrm{l})$ - Diclofenac}

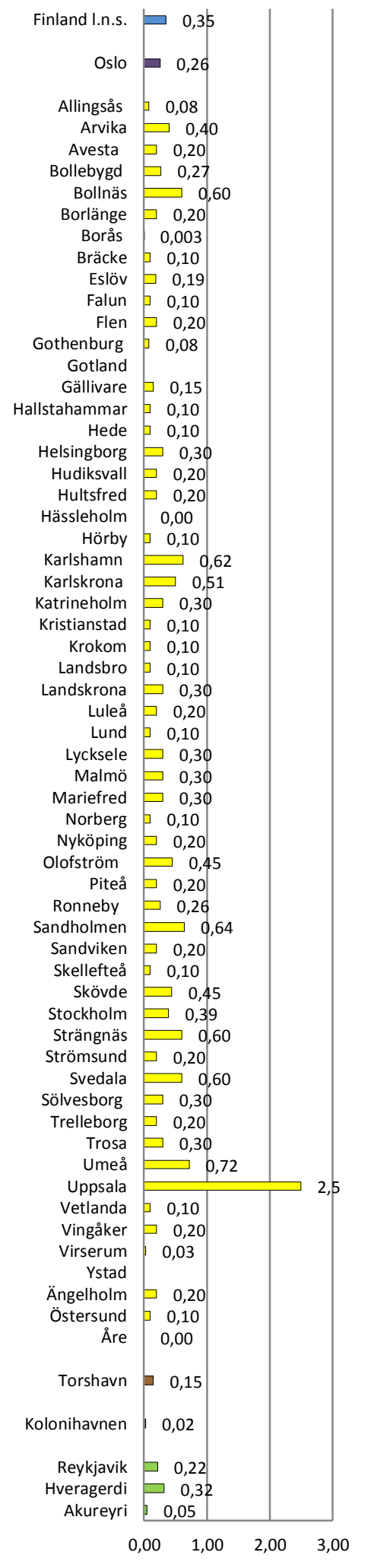




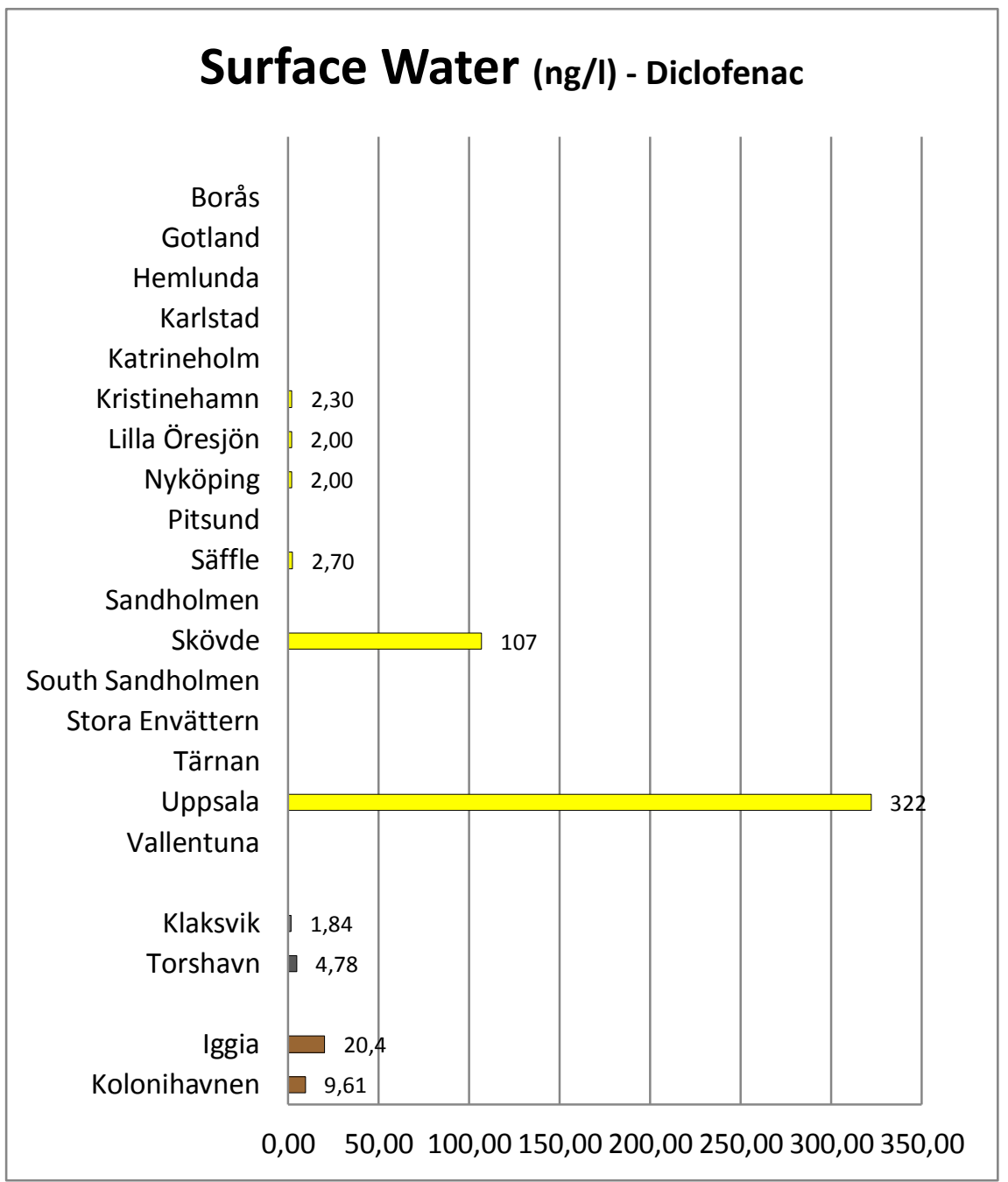




\begin{tabular}{|c|c|c|c|c|c|c|c|c|c|c|c|c|}
\hline $\begin{array}{l}\text { Diclofenac } \\
\text { Matrix }\end{array}$ & \multicolumn{2}{|c|}{ Finland } & \multicolumn{2}{|c|}{ Norway } & \multicolumn{2}{|c|}{ Sweden } & \multicolumn{2}{|c|}{ Faroe Islands } & \multicolumn{2}{|c|}{ Greenland } & \multicolumn{2}{|c|}{ Iceland } \\
\hline & $\begin{array}{r}\operatorname{Min} \\
\max \\
A D\end{array}$ & $\begin{array}{l}\text { Nr.S } \\
\text { (ref) }\end{array}$ & $\begin{array}{r}\operatorname{Min} \\
\max \\
A D\end{array}$ & $\begin{array}{l}\text { Nr.S } \\
\text { (ref) }\end{array}$ & $\begin{array}{r}\min \\
\max \\
A D\end{array}$ & $\begin{array}{l}\text { Nr.S } \\
\text { (ref) }\end{array}$ & $\begin{array}{r}\min \\
\max \\
A D\end{array}$ & $\begin{array}{l}\text { Nr.S } \\
\text { (ref) }\end{array}$ & $\begin{array}{r}\min \\
\max \\
A D\end{array}$ & $\begin{array}{l}\text { Nr.S } \\
\text { (ref) }\end{array}$ & $\begin{array}{r}\min \\
\max \\
A D\end{array}$ & $\begin{array}{l}\text { Nr.S } \\
\text { (ref) }\end{array}$ \\
\hline $\begin{array}{l}\text { Biota } \\
(\mu \mathrm{g} / \mathrm{kg} \mathrm{ww})\end{array}$ & & & & & nd & 24 (def) & & & & & & \\
\hline $\begin{array}{l}\text { Sediment } \\
(\mu \mathrm{g} / \mathrm{kg} \mathrm{dw})\end{array}$ & & & & & $\begin{array}{l}\text { nd } \\
3,5 \\
1,9\end{array}$ & $11(\mathrm{df})$ & $\begin{array}{r}0,26 \\
0,49 \\
0,4\end{array}$ & $3(\mathrm{~h})$ & $\begin{array}{r}0,18 \\
0,19 \\
0,185\end{array}$ & $2(h)$ & & \\
\hline $\begin{array}{l}\text { STP effluent } \\
\text { (ng/l) }\end{array}$ & $\begin{array}{l}\text { NA } \\
\text { NA } \\
350\end{array}$ & 14 (a) & $\begin{array}{l}160 \\
370 \\
260\end{array}$ & 6 (c) & $\begin{array}{r}\text { nd } \\
3900 \\
370\end{array}$ & 77 (efg) & $\begin{array}{l}148 \\
148 \\
148\end{array}$ & $1(\mathrm{~h})$ & $\begin{array}{l}23 \\
23 \\
23\end{array}$ & $1(\mathrm{~h})$ & $\begin{array}{r}33,4 \\
341,9 \\
190,8\end{array}$ & $5(h)$ \\
\hline $\begin{array}{l}\text { STP sludge } \\
(\mu g / k g d w)\end{array}$ & & & $\begin{array}{r}\text { nd } \\
20 \\
9,75\end{array}$ & 7 (c) & $\begin{array}{r}\text { nd } \\
560 \\
37,3\end{array}$ & 66 (ef) & $\begin{array}{l}26,9 \\
28,5 \\
27,7\end{array}$ & $2(h)$ & $\begin{array}{l}0,002 \\
0,003 \\
0,002\end{array}$ & $2(h)$ & $\begin{array}{r}1,00 \\
19,4 \\
7,8\end{array}$ & $4(h)$ \\
\hline $\begin{array}{l}\text { Surface water } \\
\text { (ng/l) }\end{array}$ & & & & & $\begin{array}{r}\text { nd } \\
880 \\
110\end{array}$ & 40 (def) & $\begin{array}{l}1 \\
8 \\
4\end{array}$ & $5(h)$ & $\begin{array}{r}2 \\
30 \\
14\end{array}$ & $5(h)$ & & \\
\hline $\begin{array}{l}\text { STP Influent } \\
\text { (ng/l) }\end{array}$ & $\begin{array}{l}\text { NA } \\
\text { NA } \\
420\end{array}$ & 14 (a) & & & $\begin{array}{r}1 \\
9700 \\
1050\end{array}$ & 35 (def) & $\begin{array}{l}110 \\
190 \\
150\end{array}$ & $2(h)$ & & & $\begin{array}{l}024 \\
690 \\
250\end{array}$ & $5(h)$ \\
\hline $\begin{array}{l}\text { Drinking water } \\
\text { (ng/l) }\end{array}$ & & & & & $\begin{array}{r}\text { nd } \\
140 \\
140\end{array}$ & $6(e)$ & & & & & & \\
\hline $\begin{array}{l}\text { Landfill leacha- } \\
\text { tes (ng/l) }\end{array}$ & & & & & $\begin{array}{l}\text { nd } \\
20 \\
15\end{array}$ & $8^{1}(\mathrm{f})$ & & & & & & \\
\hline $\begin{array}{l}\text { Settleable } \\
\text { particulate } \\
\text { material }\end{array}$ & nd & $\begin{array}{l}11 \\
\text { (b) }\end{array}$ & & & & & & & & & & \\
\hline Manure & & & & & nd & 5 (f) & & & & & & \\
\hline $\begin{array}{l}\text { Hospital WW } \\
\text { (ng/l) }\end{array}$ & & & & & $\begin{array}{r}\text { nd } \\
5 \\
1,4\end{array}$ & $7(f)$ & $\begin{array}{l}0,138 \\
0,597 \\
0,367\end{array}$ & $2(\mathrm{~h})$ & $\begin{array}{l}0,014 \\
0,014 \\
0,014\end{array}$ & $1(\mathrm{~h})$ & & \\
\hline
\end{tabular}

\section{References:}

a) (Vieno, 2008)

b) (Lahti \& Oikari, 2011)

c) (Thomas, et al., 2007)

d) (Remberger, et al., 2009)

e) (Fick, et al., 2011);

f) (Andersson, et al., 2006)

g) (Lilja, et al., 2010)

h) (Huber, et al., NA) 


\section{Conclusion:}

The compound was monitored in two Finnish, one Norwegian study, four Swedish studies, and one international study covering Faroe Isalands, Greenland and Iceland. The compound was detected in several matrices:

- In sediment in Sweden, Faroe Islands and Greenland. The highest values were detected in Sweden

- In STP effluent samples in all countries

- In STP sludge in all the countries except Finland were the compound was not monitored in sludge. The values detected were significantly higher in Sweden

- In surface water in Sweden, Faroe Islands and Greenland. The highest values were detected in Sweden

- In landfill leachates in Sweden

- In STP influent in Sweden, Finland, Faroe Islands and Iceland. The values detected were significantly higher in Sweden

- In hospital waste water in Sweden, Faroe Island and Greenland

- In one drinking water sample in Sweden

\subsubsection{Ibuprofen}

CAS nr.: 15687-27-1

Info: A nonsteroidal anti-inflammatory drug also used as an analgesic

Toxicity data:

NOEC 7 days (Lemna gibba) $>1000 \mu \mathrm{g} / \mathrm{l}$

NOEC $96 \mathrm{hr}$ (Lepomis macrochirus) $=10 \mathrm{mg} / \mathrm{l}$

NOEC $48 \mathrm{~h}$ (Daphnia Magna) $=3.37 \mathrm{mg} / \mathrm{l}$

EC50 $96 \mathrm{hr}$ (Skeletonema costatum) $=7.1 \mathrm{mg} / \mathrm{l}$

$P N E C=71 \mu \mathrm{g} / \mathrm{l}$ (Andersson, et al., 2006)

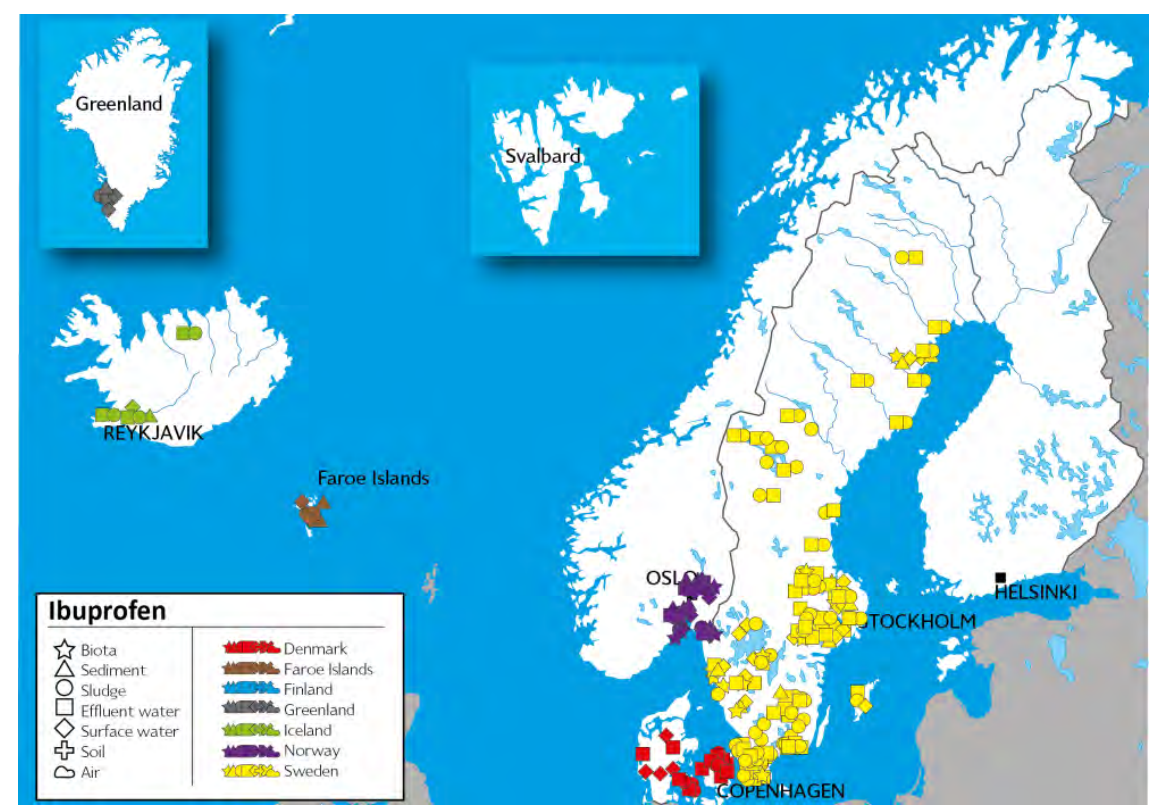




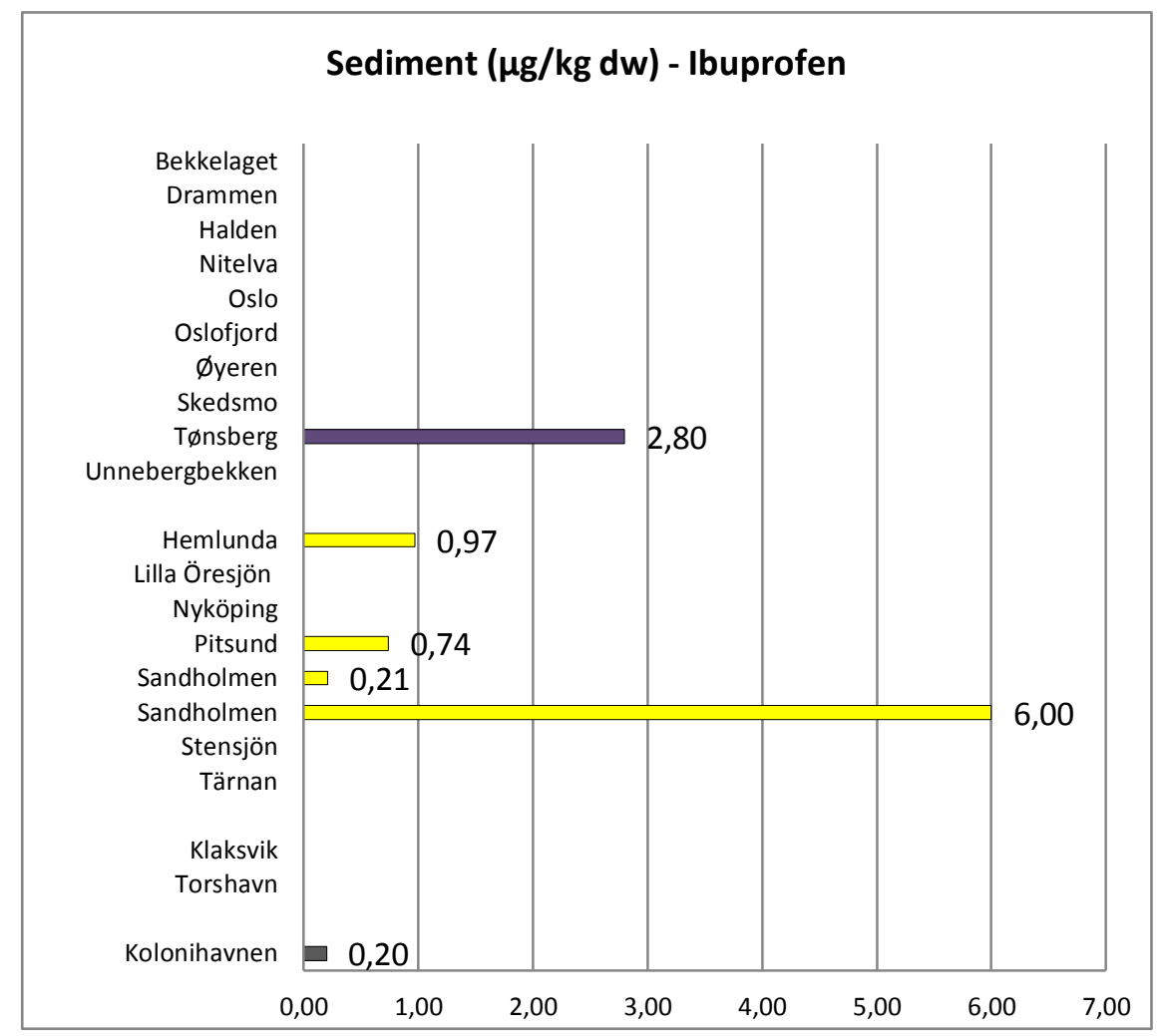



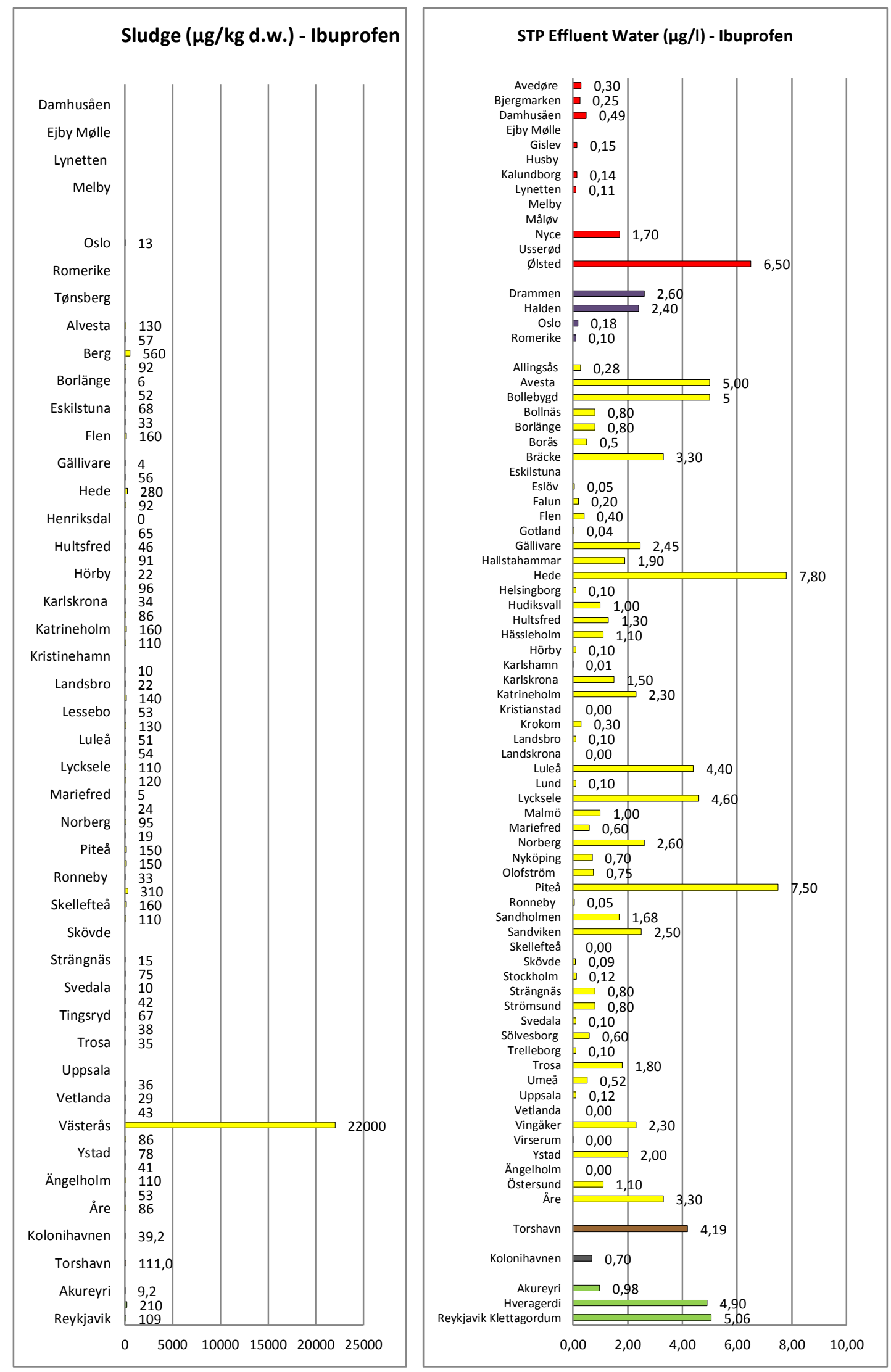


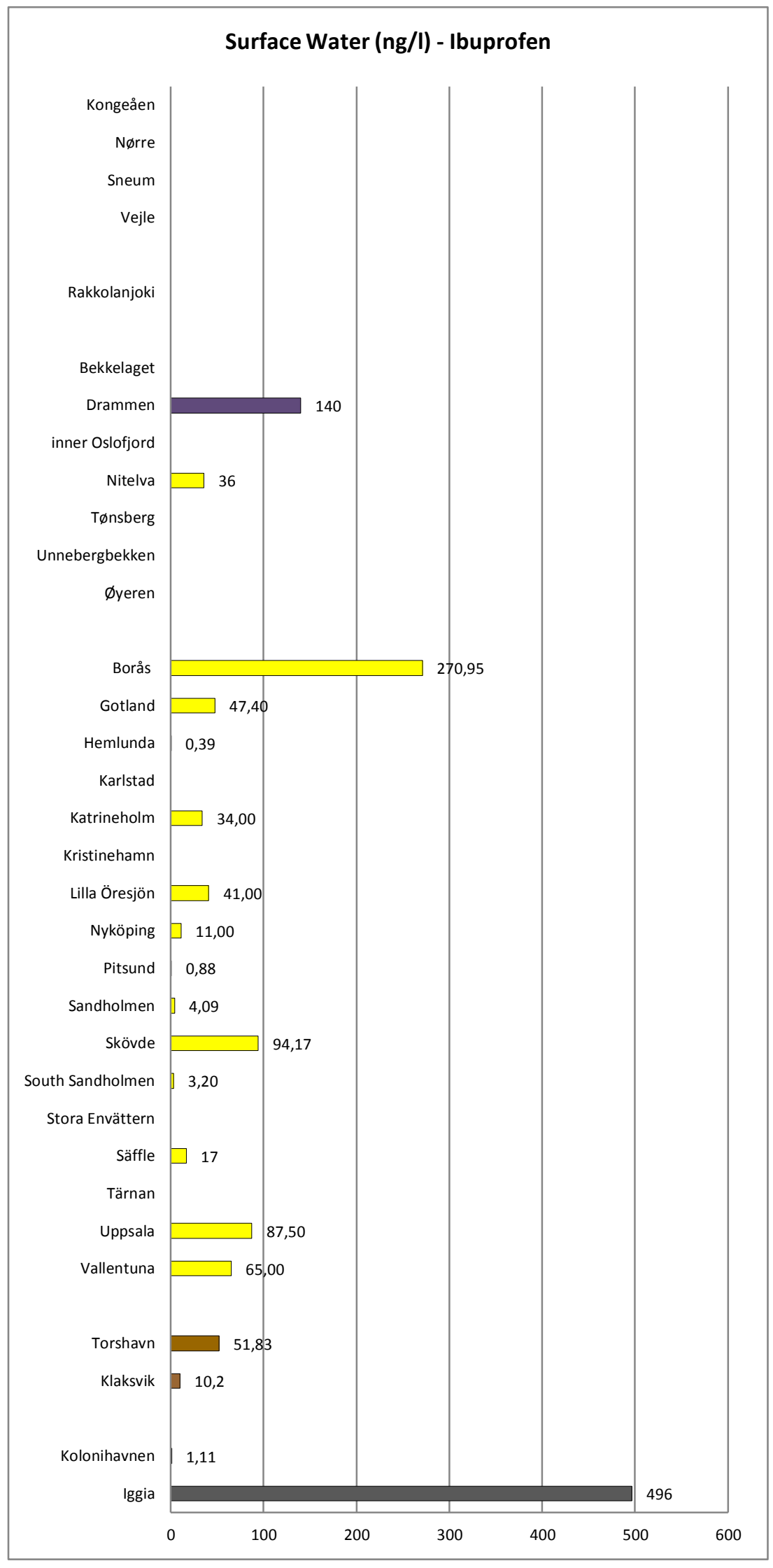




\begin{tabular}{|c|c|c|c|c|c|c|c|c|c|c|c|c|c|c|}
\hline \multirow{2}{*}{$\begin{array}{l}\text { Ibuprofen } \\
\text { Matrix }\end{array}$} & \multicolumn{2}{|c|}{ Denmark } & \multicolumn{2}{|c|}{ Finland } & \multicolumn{2}{|c|}{ Norway } & \multicolumn{2}{|c|}{ Sweden } & \multicolumn{2}{|c|}{ Faroe Islands } & \multicolumn{2}{|c|}{ Greenland } & \multicolumn{2}{|c|}{ Iceland } \\
\hline & $\begin{array}{r}\operatorname{Min} \\
\max \\
A D\end{array}$ & $\begin{array}{l}\text { Nr.S } \\
\text { (ref) }\end{array}$ & $\begin{array}{r}\min \\
\max \\
A D\end{array}$ & $\begin{array}{l}\text { Nr.S } \\
\text { (ref) }\end{array}$ & $\begin{array}{r}\min \\
\max \\
A D\end{array}$ & $\begin{array}{l}\text { Nr.S } \\
\text { (ref) }\end{array}$ & $\begin{array}{r}\min \\
\max \\
A D\end{array}$ & $\begin{array}{l}\text { Nr.S } \\
\text { (ref) }\end{array}$ & $\begin{array}{r}\min \\
\max \\
A D\end{array}$ & $\begin{array}{l}\text { Nr.S } \\
\text { (ref) }\end{array}$ & $\begin{array}{r}\min \\
\max \\
A D\end{array}$ & $\begin{array}{l}\text { Nr.S } \\
\text { (ref) }\end{array}$ & $\begin{array}{r}\min \\
\max \\
A D\end{array}$ & $\begin{array}{l}\text { Nr.S } \\
\text { (ref) }\end{array}$ \\
\hline Biota ( $\mu \mathrm{g} / \mathrm{kg} w w)$ & & & & & nd & 6 (b) & nd & 24 (def) & & & & & & \\
\hline $\begin{array}{l}\text { Sediment } \\
(\mu \mathrm{g} / \mathrm{kg} \mathrm{dw})\end{array}$ & & & & & $\begin{array}{l}\text { nd } \\
2,8 \\
2,8\end{array}$ & 16 (b) & $\begin{array}{r}\text { nd } \\
6 \\
1,98\end{array}$ & 11 (ef) & nd & $3(k)$ & $\begin{array}{r}0,18 \\
0,21 \\
0,198\end{array}$ & $2(k)$ & & \\
\hline $\begin{array}{l}\text { STP sludge } \\
(\mu \mathrm{g} / \mathrm{kg} \mathrm{dw})\end{array}$ & nd & $10(a)$ & & & $\begin{array}{r}\mathrm{nd} \\
17 \\
12,5\end{array}$ & $13(\mathrm{bc})$ & $\begin{array}{r}\text { nd } \\
22000 \\
450,9\end{array}$ & 66 (df) & $\begin{array}{r}53,5 \\
169 \\
111\end{array}$ & $2(k)$ & $\begin{array}{l}30,1 \\
48,2 \\
39,2\end{array}$ & $2(k)$ & $\begin{array}{r}2,6 \\
209,5 \\
84,2\end{array}$ & $4(k)$ \\
\hline $\begin{array}{l}\text { Surface water } \\
(\mu \mathrm{g} / \mathrm{l})\end{array}$ & nd & 16 (a) & & & $\begin{array}{r}\text { nd } \\
0,14 \\
0,09\end{array}$ & 8 (b) & $\begin{array}{r}\text { nd } \\
0,18 \\
0,05\end{array}$ & 32 (def) & $\begin{array}{r}0,003 \\
0,13 \\
0,044\end{array}$ & $5(k)$ & $\begin{array}{r}\text { nd } \\
0,87 \\
0,25\end{array}$ & $5(k)$ & & \\
\hline $\begin{array}{l}\text { STP effluent } \\
(\mu \mathrm{g} / \mathrm{l})\end{array}$ & $\begin{array}{l}\text { nd } \\
6,5 \\
1,5\end{array}$ & 8 (ah) & $\begin{array}{r}N A \\
N A \\
2,06\end{array}$ & 14 (i) & $\begin{array}{r}\text { nd } \\
2,6 \\
0,75\end{array}$ & 10 (bc) & $\begin{array}{l}\text { nd } \\
7,8 \\
1,3\end{array}$ & $\begin{array}{r}82 \\
\text { (defgh) }\end{array}$ & $\begin{array}{l}4,19 \\
4,19 \\
4,19\end{array}$ & $1(\mathrm{k})$ & $\begin{array}{l}0,7 \\
0,7 \\
0,7\end{array}$ & $1(\mathrm{k})$ & $\begin{array}{l}0,64 \\
5,08 \\
3,36\end{array}$ & $5(k)$ \\
\hline $\begin{array}{l}\text { Hospital WW } \\
(\mu \mathrm{g} / \mathrm{l})\end{array}$ & & & & & & & $\begin{array}{r}0,0034 \\
12,1 \\
10,7\end{array}$ & $7(f)$ & $\begin{array}{r}3,38 \\
4,5 \\
3,94\end{array}$ & $2(k)$ & $\begin{array}{l}2,81 \\
2,81 \\
2,81\end{array}$ & $1(k)$ & & \\
\hline Drinking water & & & & & & & nd & 6 (d) & & & & & & \\
\hline $\begin{array}{l}\text { Landfill leachates } \\
\text { untreated }(\mu \mathrm{g} / \mathrm{l})\end{array}$ & & & & & $\begin{array}{r}\text { nd } \\
1,3 \\
1,15\end{array}$ & 4 (b) & $\begin{array}{l}1,1 \\
1,1 \\
1,1\end{array}$ & $1(f)$ & & & & & & \\
\hline $\begin{array}{l}\text { Landfill leachates } \\
\text { treated }(\mu \mathrm{g} / \mathrm{l})\end{array}$ & & & & & $\begin{array}{r}0,61 \\
1,8 \\
1,2\end{array}$ & 5 (b) & $\begin{array}{r}\text { nd } \\
1,6 \\
0,76\end{array}$ & $7(f)$ & & & & & & \\
\hline $\begin{array}{l}\text { Warning system } \\
\text { for pesticide } \\
\text { leaching to } \\
\text { groundwater }\end{array}$ & nd & 6 (a) & & & & & & & & & & & & \\
\hline Manure & & & & & & & nd & $5(f)$ & & & & & & \\
\hline Pig slurry & nd & $4(a)$ & & & & & & & & & & & & \\
\hline Drain water & nd & 1 (a) & & & & & & & & & & & & \\
\hline Groundwater & nd & 3 (a) & & & & & & & & & & & & \\
\hline STP Influent ( $\mu \mathrm{g} / \mathrm{l})$ & $\begin{array}{r}4,4 \\
16 \\
7,9\end{array}$ & 9 (a) & $\begin{array}{r}N A \\
N A \\
0,16\end{array}$ & 14 (i) & $\begin{array}{r}\text { nd } \\
3,05 \\
2,6\end{array}$ & 8 (b) & $\begin{array}{r}0,0013 \\
11,8 \\
4,8\end{array}$ & 35 (def) & $\begin{array}{r}3,5 \\
11,47 \\
7,5\end{array}$ & $2(k)$ & & & $\begin{array}{r}0,0016 \\
5,04 \\
2,14\end{array}$ & $5(k)$ \\
\hline $\begin{array}{l}\text { Settleable particu- } \\
\text { late material } \\
(\mathrm{ng} / \mathrm{g} \mathrm{dw})\end{array}$ & & & $\begin{array}{r}\text { nd } \\
152 \\
30,2\end{array}$ & $11(\mathrm{j})$ & & & & & & & & & & \\
\hline
\end{tabular}

\section{References:}

a) (Mogensen, et al., 2008)

b) (Møskeland, et al., 2006)

c) (Thomas, et al., 2007)

d) (Fick, et al., 2011)

e) (Remberger, et al., 2009)

f) (Andersson, et al., 2006) 
g) (Lilja, et al., 2010)

h) (Kjølholt, et al., 2003)

i) (Vieno, 2008)

j) (Lahti \& Oikari, 2011)

k) (Huber, et al., NA)

\section{Conclusion:}

The compound was broadly monitored in the nordic countries, in one study in Denmark, two in Norway and Finland, five in Sweden and one international study covering Faroe Islands, Greenland and Iceland. The compound was detected in several matrices:

- Surface water in Norway, Sweden, Faroe Islands and Greenland. But was not detected in surface water in Denmark. Detected in concentrations higher than the PNEC in Sweden

- Sediment in all countries monitored, with the exception of Denmark and Finland

- STP sludge in Norway, Sweden, Faroe Islands, Greenland and Iceland. But was not detected in Denmark. One very high value detected in Sweden

- STP effluent in all four countries

- Landfill leachates

- STP influent in all countries

- Hospital waste water in Sweden 


\subsubsection{Ketoprofen}

CAS nr.: 22071-15-4

Info: Propionic acid class of non-steroidal anti-inflammatory drug with analgesic and antipyretic effects

Toxicity data: $N A$

$P N E C=N A$
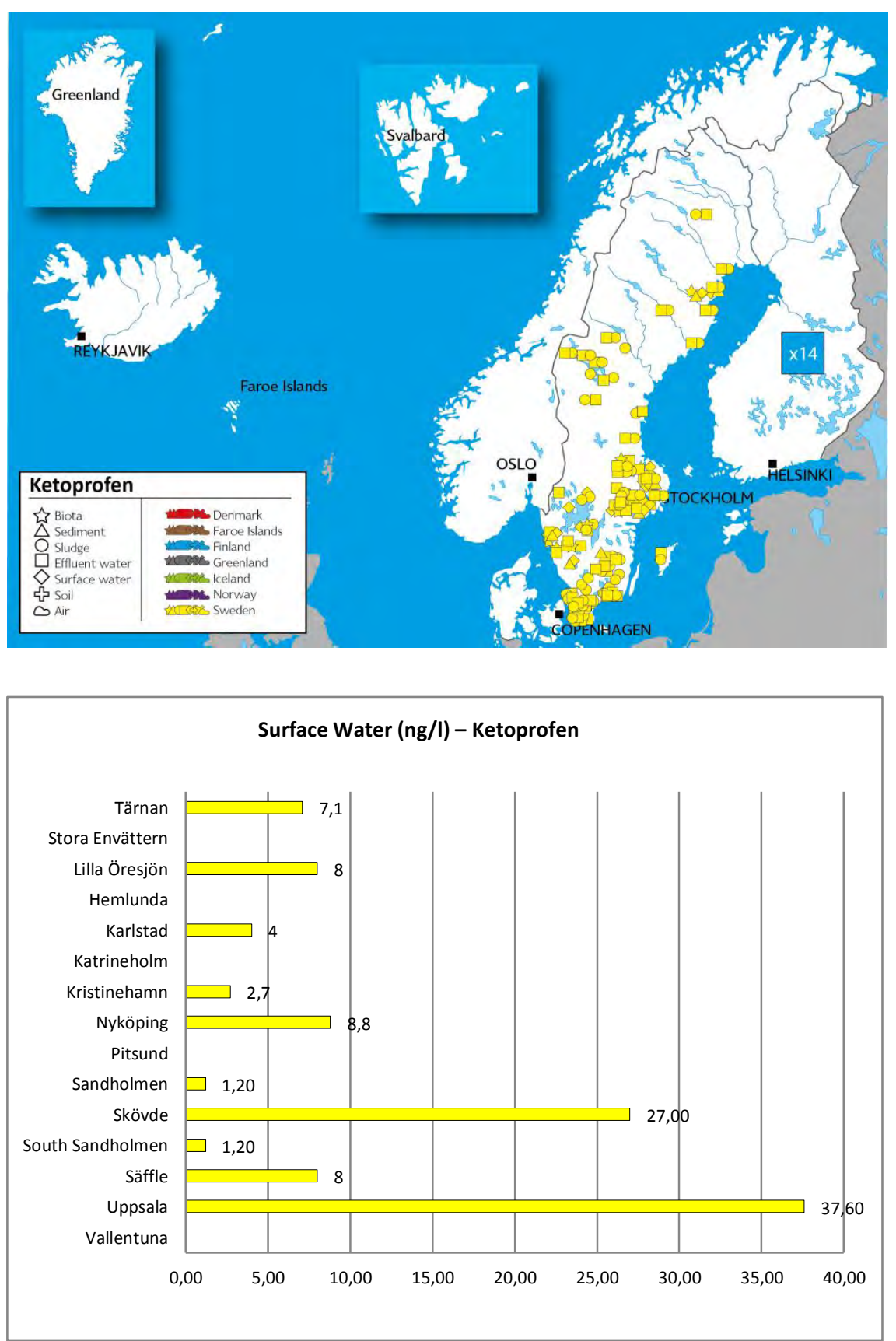


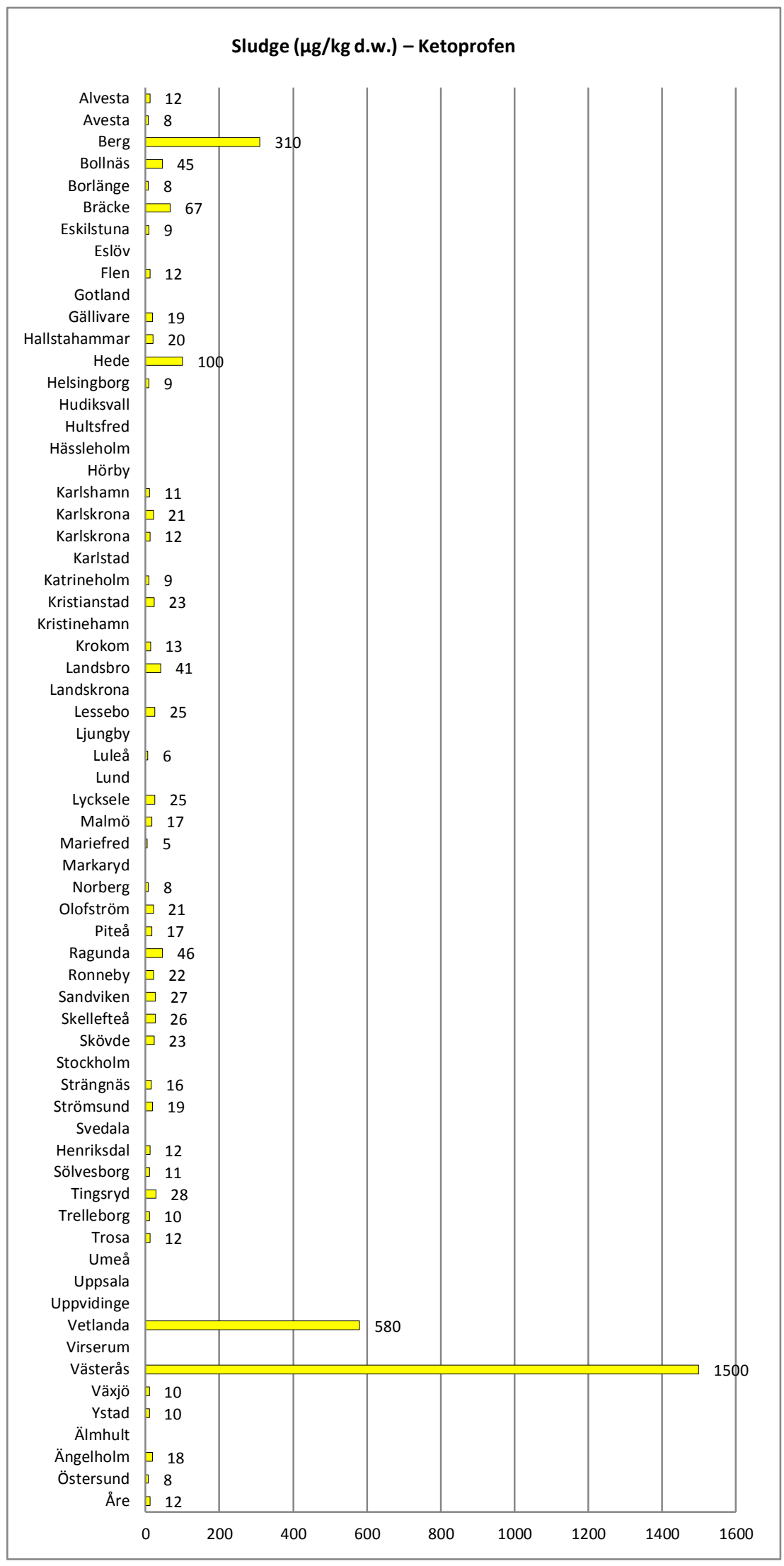




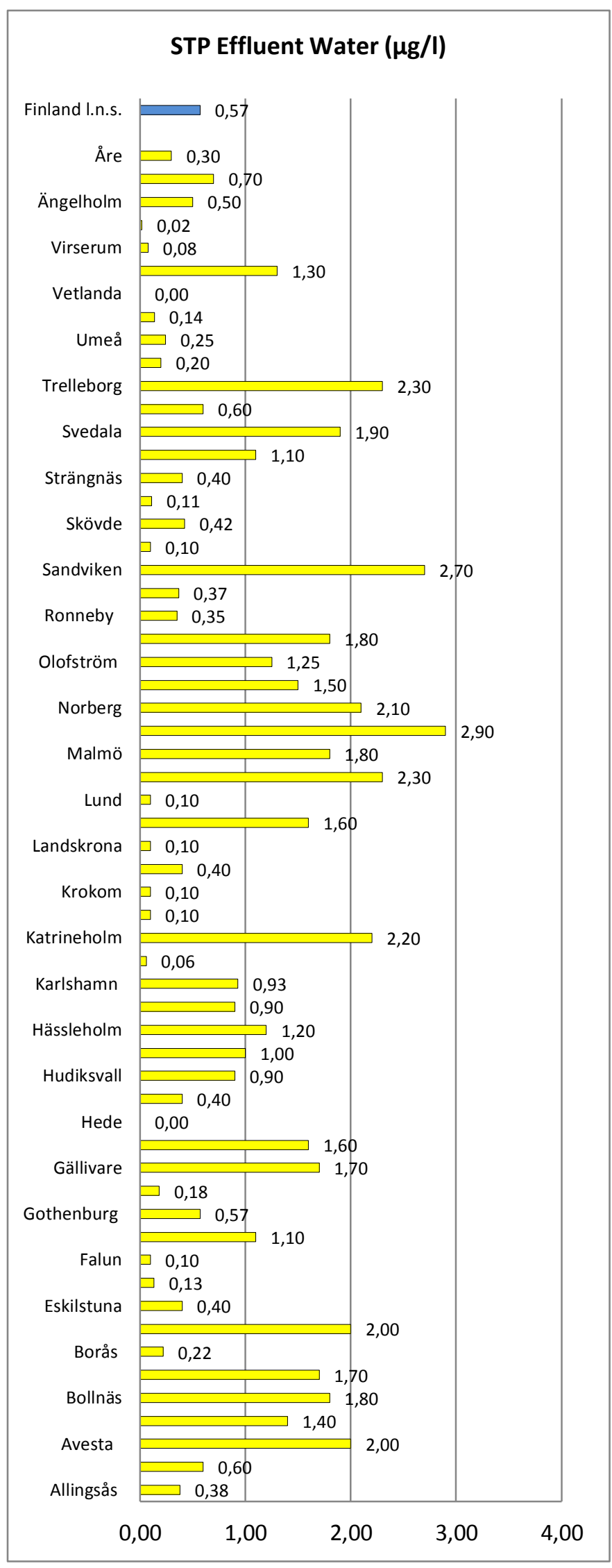




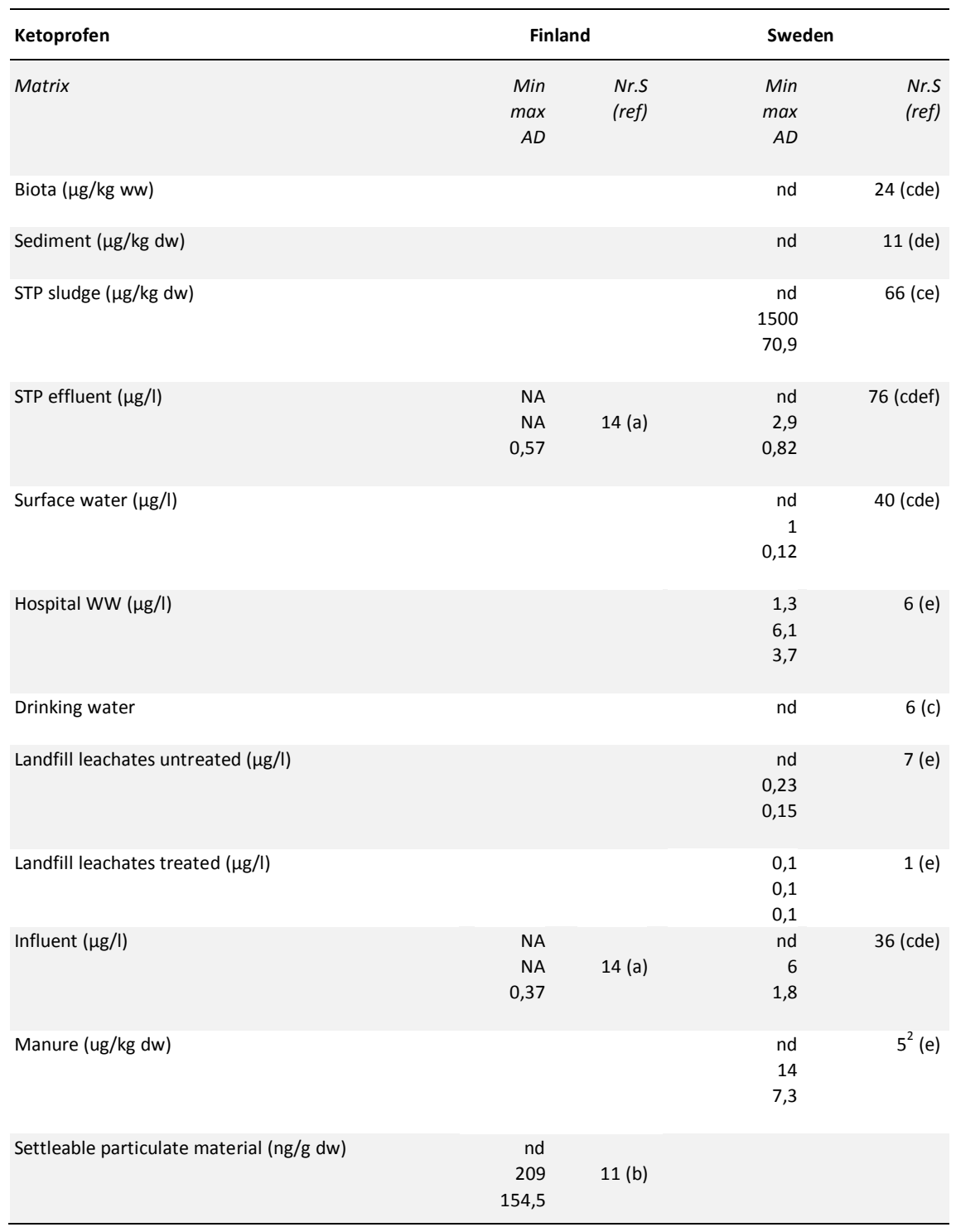

\section{References:}

a) (Vieno, 2008)

b) (Lahti \& Oikari, 2011)

c) (Fick, et al., 2011)

d) (Remberger, et al., 2009)

e) (Andersson, et al., 2006)

f) (Lilja, et al., 2010) 


\section{Conclusion:}

The compound was monitored in two Finnish studies and four Swedish studies. It was detected in several matrices:

- In STP sludge in high concentrations in Sweden

- In STP effluent in both countries

- In surface water in Sweden

- In landfill leachates in Sweden

- In manure in Sweden

- In settleable particulate material in Finland

- In STP influent in both countries

- In hospital waste water in Sweden

\subsubsection{Naproxen}

CAS nr.: 22204-53-1

Info: A nonsteroidal anti-inflammatory drug with analgesic and antipyretic effects

Toxicity data: LOEC (Ceriodaphnia dubia) $=32 \mu \mathrm{g} / \mathrm{L}$ (Schlabach, et al., 2009) $P N E C=35 \mu \mathrm{g} / \mathrm{l}$ (Andersson, et al., 2006)

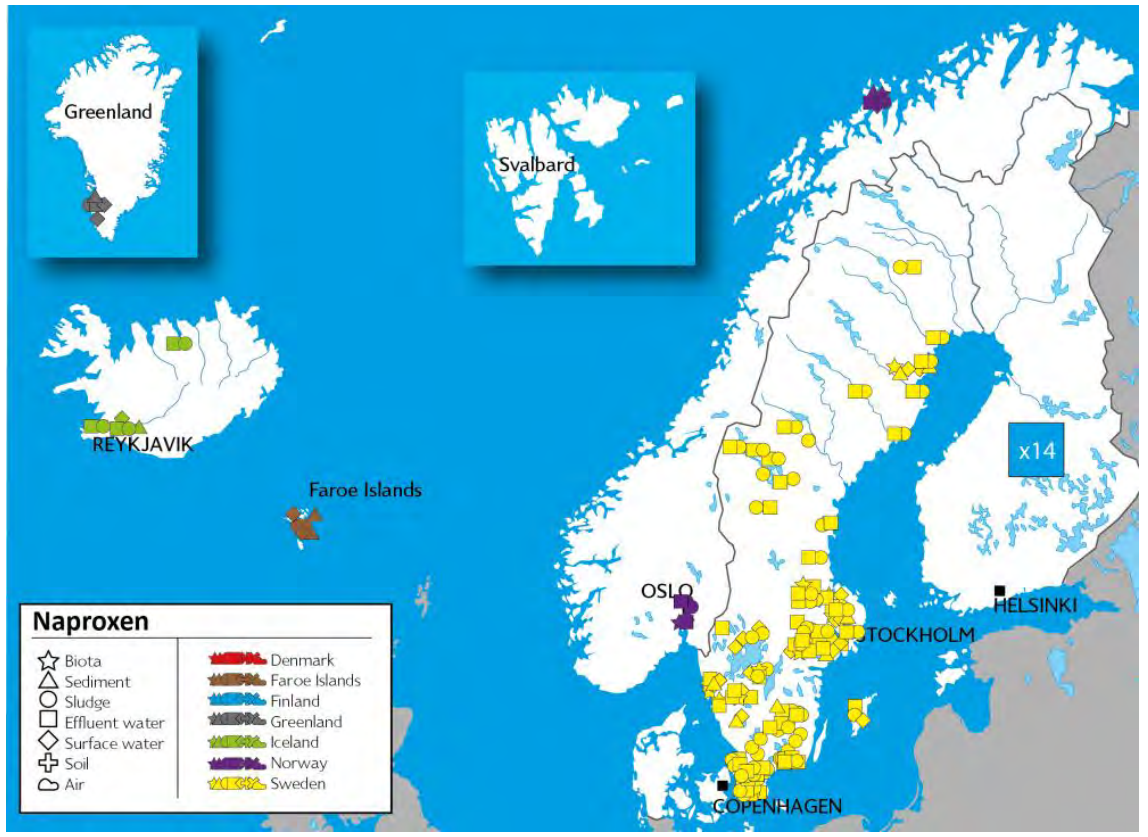




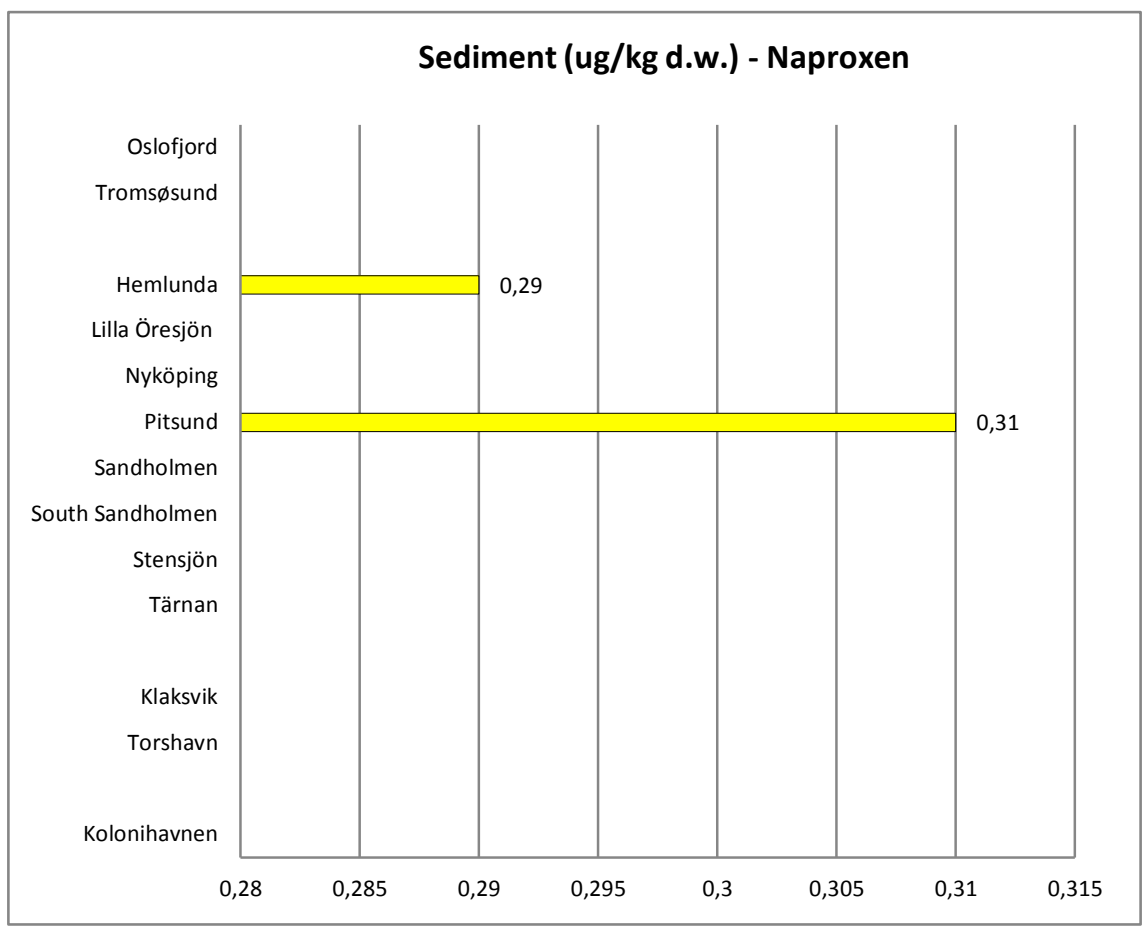


Sludge (ug/kg d.w.) - Naproxen

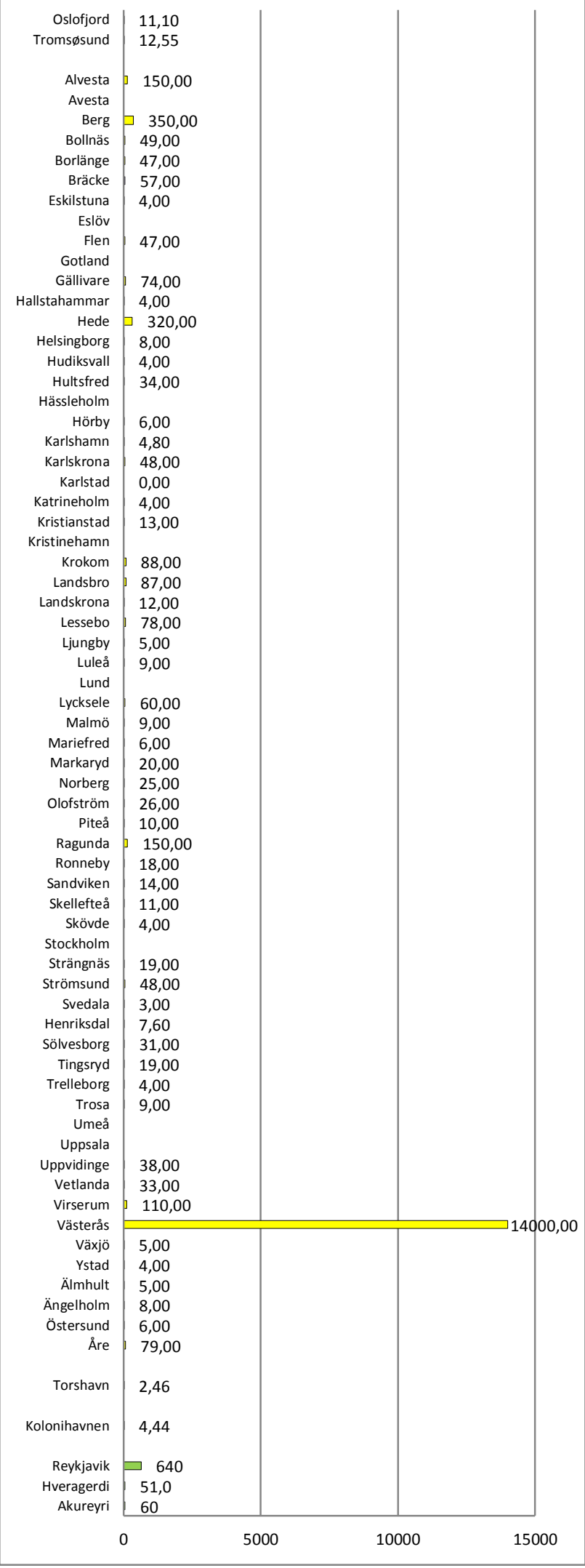




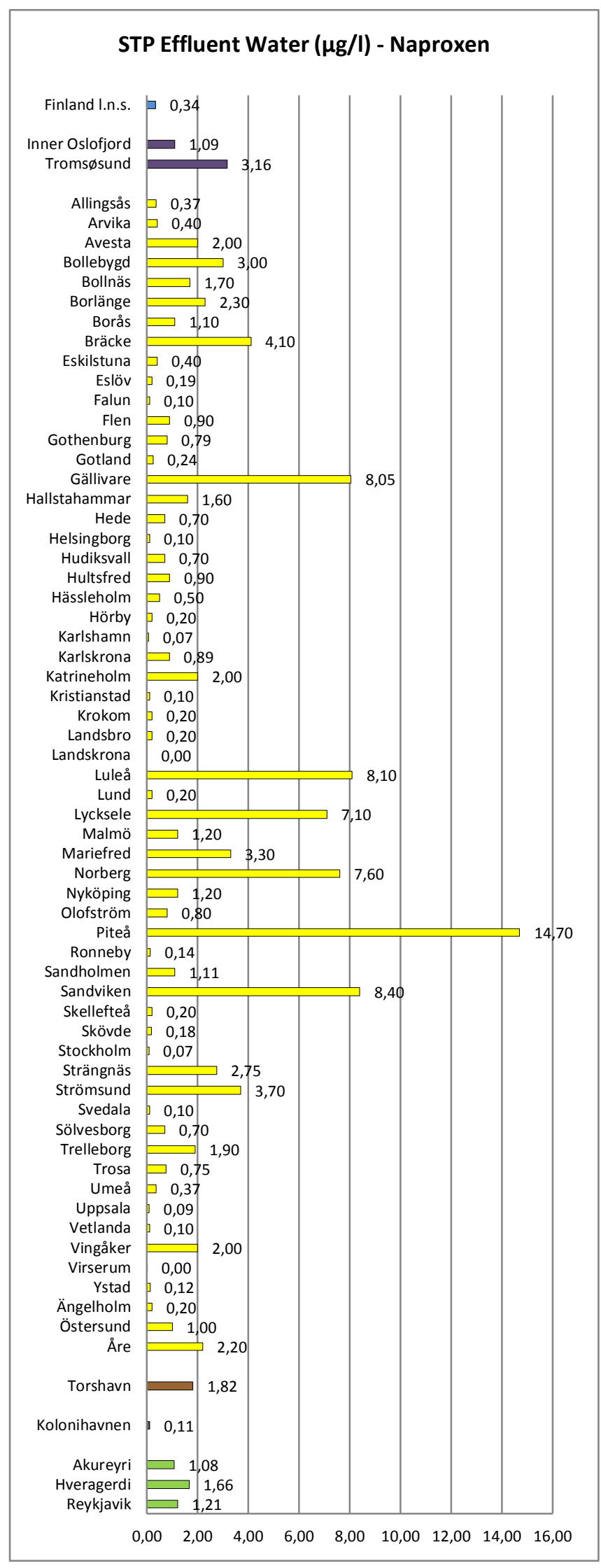




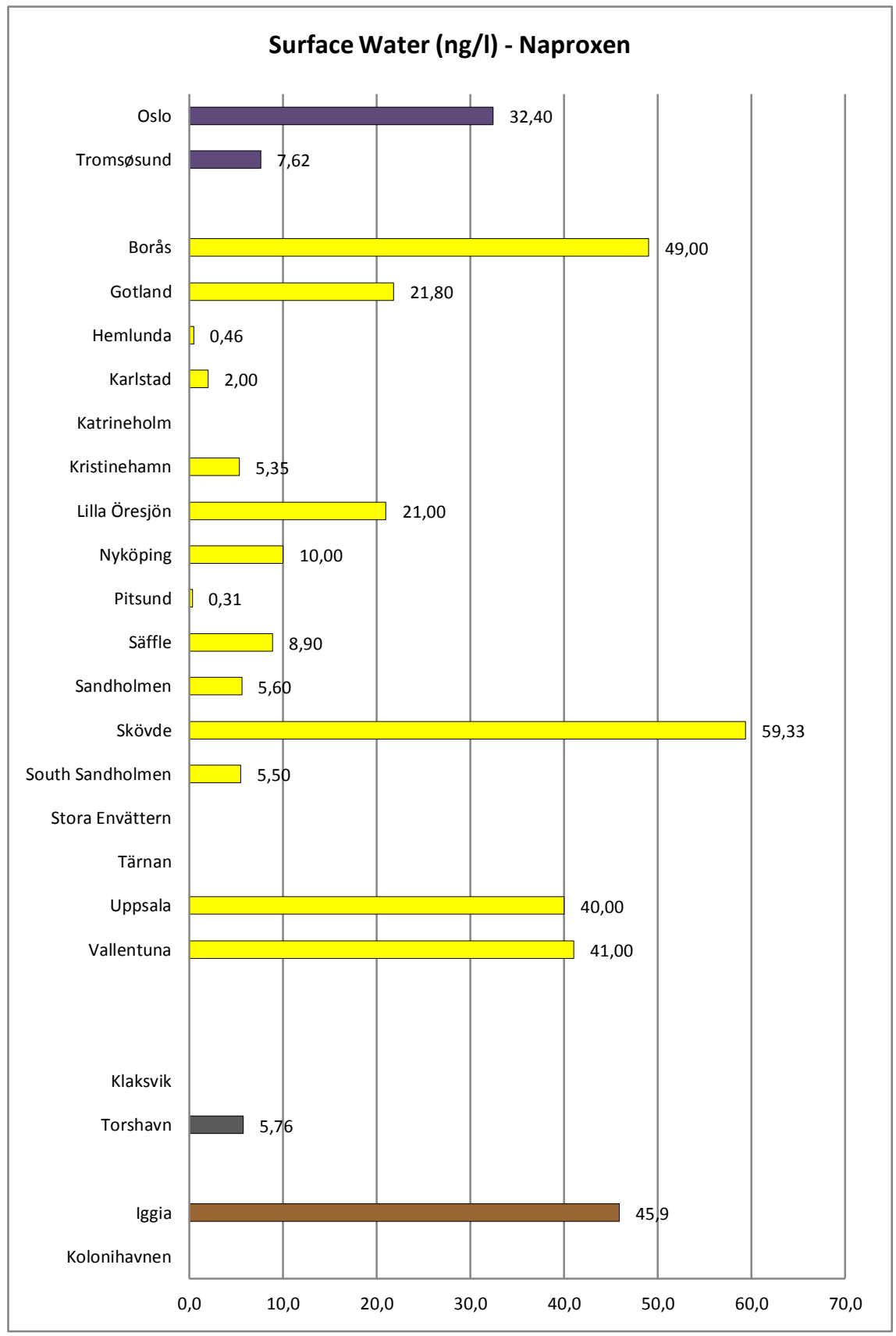




\begin{tabular}{|c|c|c|c|c|c|c|c|c|c|c|c|c|}
\hline \multirow{2}{*}{$\begin{array}{l}\text { Naproxen } \\
\text { Matrix }\end{array}$} & \multicolumn{2}{|c|}{ Finland } & \multicolumn{2}{|c|}{ Norway } & \multicolumn{2}{|c|}{ Sweden } & \multicolumn{2}{|c|}{ Faroe Islands } & \multicolumn{2}{|c|}{ Greenland } & \multicolumn{2}{|c|}{ Iceland } \\
\hline & $\begin{array}{r}\operatorname{Min} \\
\max \\
A D\end{array}$ & $\begin{array}{l}\text { Nr.S } \\
\text { (ref) }\end{array}$ & $\begin{array}{r}\min \\
\max \\
A D\end{array}$ & $\begin{array}{l}\text { Nr.S } \\
\text { (ref) }\end{array}$ & $\begin{array}{r}\min \\
\max \\
A D\end{array}$ & $\begin{array}{l}\text { Nr.S } \\
\text { (ref) }\end{array}$ & $\begin{array}{r}\min \\
\max \\
A D\end{array}$ & $\begin{array}{l}\text { Nr.S } \\
\text { (ref) }\end{array}$ & $\begin{array}{r}\min \\
\max \\
A D\end{array}$ & $\begin{array}{l}\text { Nr.S } \\
\text { (ref) }\end{array}$ & $\begin{array}{r}\min \\
\max \\
A D\end{array}$ & $\begin{array}{l}\text { Nr.S } \\
\text { (ref) }\end{array}$ \\
\hline $\begin{array}{l}\text { Biota } \\
(\mu \mathrm{g} / \mathrm{kg} w w)\end{array}$ & & & $\begin{array}{l}\text { nd } \\
\text { nd } \\
\text { nd }\end{array}$ & $3(c)$ & $\begin{array}{l}\text { nd } \\
\text { nd } \\
\text { nd }\end{array}$ & 24 (de) & & & & & & \\
\hline $\begin{array}{l}\text { Sediment } \\
(\mu \mathrm{g} / \mathrm{kg} \mathrm{dw})\end{array}$ & & & $\begin{array}{l}\text { nd } \\
\text { nd } \\
\text { nd }\end{array}$ & 6 (c) & $\begin{array}{r}\text { nd } \\
0,31 \\
0,30\end{array}$ & 11 (ef) & nd & $3(h)$ & nd & $5(h)$ & & \\
\hline $\begin{array}{l}\text { STP sludge } \\
(\mu \mathrm{g} / \mathrm{kg} \mathrm{dw})\end{array}$ & & & $\begin{array}{r}8,1 \\
17 \\
11,8\end{array}$ & 4 (c) & $\begin{array}{r}\text { nd } \\
14000 \\
296,9\end{array}$ & 66 (df) & $\begin{array}{r}0,32 \\
4,6 \\
2,46\end{array}$ & $2(h)$ & $\begin{array}{l}0,87 \\
8,01 \\
4,44\end{array}$ & 2 (h) & $\begin{array}{r}0,8 \\
640,3 \\
203,1\end{array}$ & $4(h)$ \\
\hline $\begin{array}{l}\text { STP effluent } \\
(\mu \mathrm{g} / \mathrm{l})\end{array}$ & $\begin{array}{r}N A \\
N A \\
0,34\end{array}$ & 14 (a) & $\begin{array}{r}\text { nd } \\
3,16 \\
1,32\end{array}$ & $10(c)$ & $\begin{array}{r}0,0003 \\
14,7 \\
1,56\end{array}$ & 76 (defg) & $\begin{array}{l}1,8 \\
1,8 \\
1,8\end{array}$ & $1(\mathrm{~h})$ & $\begin{array}{l}0,106 \\
0,106 \\
0,106\end{array}$ & $1(\mathrm{~h})$ & $\begin{array}{l}0,52 \\
1,92 \\
1,34\end{array}$ & $5(h)$ \\
\hline $\begin{array}{l}\text { Surface water } \\
(\mu \mathrm{g} / \mathrm{l})\end{array}$ & & & $\begin{array}{r}0,003 \\
0,053 \\
0,02\end{array}$ & 12 (c) & $\begin{array}{r}\text { nd } \\
0,12 \\
0,02\end{array}$ & 40 (def) & $\begin{array}{r}\text { nd } \\
0,0058 \\
0,0058\end{array}$ & $5(h)$ & $\begin{array}{r}\text { nd } \\
0,046 \\
0,046\end{array}$ & $5(h)$ & & \\
\hline $\begin{array}{l}\text { Hospital WW } \\
(\mu \mathrm{g} / \mathrm{l})\end{array}$ & & & & & $\begin{array}{r}\text { nd } \\
11 \\
4,87\end{array}$ & 7 (f) & $\begin{array}{r}\text { nd } \\
7,88 \\
7,88\end{array}$ & $2(h)$ & $\begin{array}{l}0,0035 \\
0,0035 \\
0,0035\end{array}$ & 1 (h) & & \\
\hline $\begin{array}{l}\text { Drinking water } \\
(\mu \mathrm{g} / \mathrm{l})\end{array}$ & & & & & $\begin{array}{r}\text { nd } \\
0,045 \\
0,04\end{array}$ & 6 (d) & & & & & & \\
\hline $\begin{array}{l}\text { Landfill leac- } \\
\text { hates untreat- } \\
\text { ed }(\mu \mathrm{g} / \mathrm{l})\end{array}$ & & & & & $\begin{array}{r}\text { nd } \\
0,02 \\
0,015\end{array}$ & $8(f)$ & & & & & & \\
\hline $\begin{array}{l}\text { Landfill leac- } \\
\text { hates treated } \\
(\mu \mathrm{g} / \mathrm{l})\end{array}$ & & & & & $\begin{array}{l}\text { nd } \\
\text { nd } \\
\text { nd }\end{array}$ & $1(f)$ & & & & & & \\
\hline Influent $(\mu \mathrm{g} / \mathrm{l})$ & $\begin{array}{r}N A \\
N A \\
0,69\end{array}$ & 14 (a) & & & $\begin{array}{r}0,0002 \\
20,9 \\
4,33\end{array}$ & 35 (def) & $\begin{array}{r}0,0027 \\
0,17 \\
0,086\end{array}$ & $2(h)$ & & & $\begin{array}{r}0,175 \\
2,3 \\
1,67\end{array}$ & $5(h)$ \\
\hline Manure & & & & & $\begin{array}{l}\text { nd } \\
\text { nd } \\
\text { nd }\end{array}$ & $5(f)$ & & & & & & \\
\hline $\begin{array}{l}\text { Settleable par- } \\
\text { ticulate ma- } \\
\text { terial (ng/g dw) }\end{array}$ & $\begin{array}{r}\text { nd } \\
49,2 \\
49,2\end{array}$ & 11 (b) & & & & & & & & & & \\
\hline
\end{tabular}

References:
a) (Vieno, 2008)
b) (Lahti \& Oikari, 2011)
c) (Schlabach, et al., 2009)
d) (Fick, et al., 2011)
e) (Remberger, et al., 2009)
f) (Andersson, et al., 2006)
g) (Lilja, et al., 2010)
h) (Huber, et al., NA) 


\section{Conclusion:}

The compound has been monitored in one Norwegian study, two Finnish studies, four Swedish studies, and one international study covering Faroe Islands, Greenland, and Iceland. It has been detected in several matrices:

- Sediment only in Sweden

- Sludge in all countries except Finland were the compound was not monitored. A very high value was detected in one location in Sweden.

- STP effluent in all countries, in concentrations below the PNEC

- Surface water in Sweden, Norway, Faroe Islands and Greenland, in concentrations below the PNEC

- In one sample of settleable particulate material in Finland

- Non-treated landfill leachates in Sweden but not in treated leachates

- STP influent in all countries monitored in all countries monitored

- Hospital waste water

\subsubsection{Paracetamol}

CAS nr.: 103-90-2

Info: Analgesic and antipyretic

Toxicity data: $\mathrm{LOEC}$ (Lemna gibba) $=1000 \mu \mathrm{g} / \mathrm{L}$ (Schlabach, et al., 2009)

$P N E C=N A$

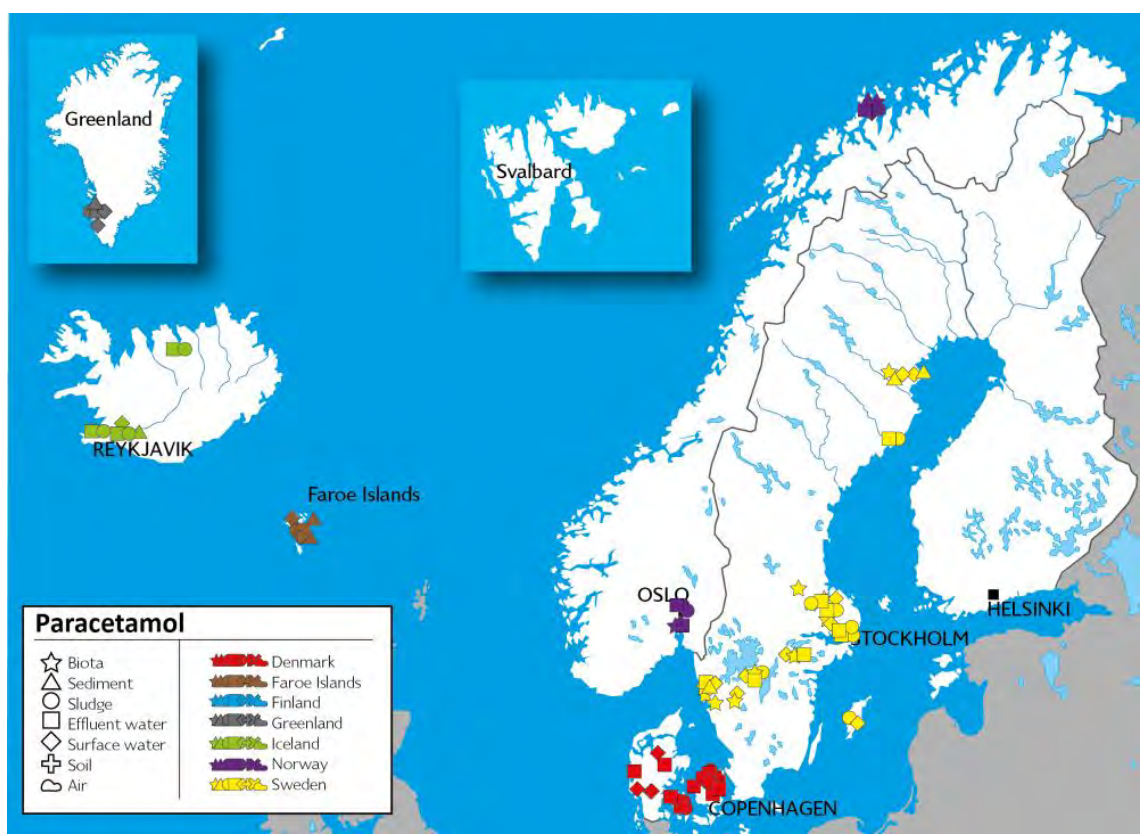



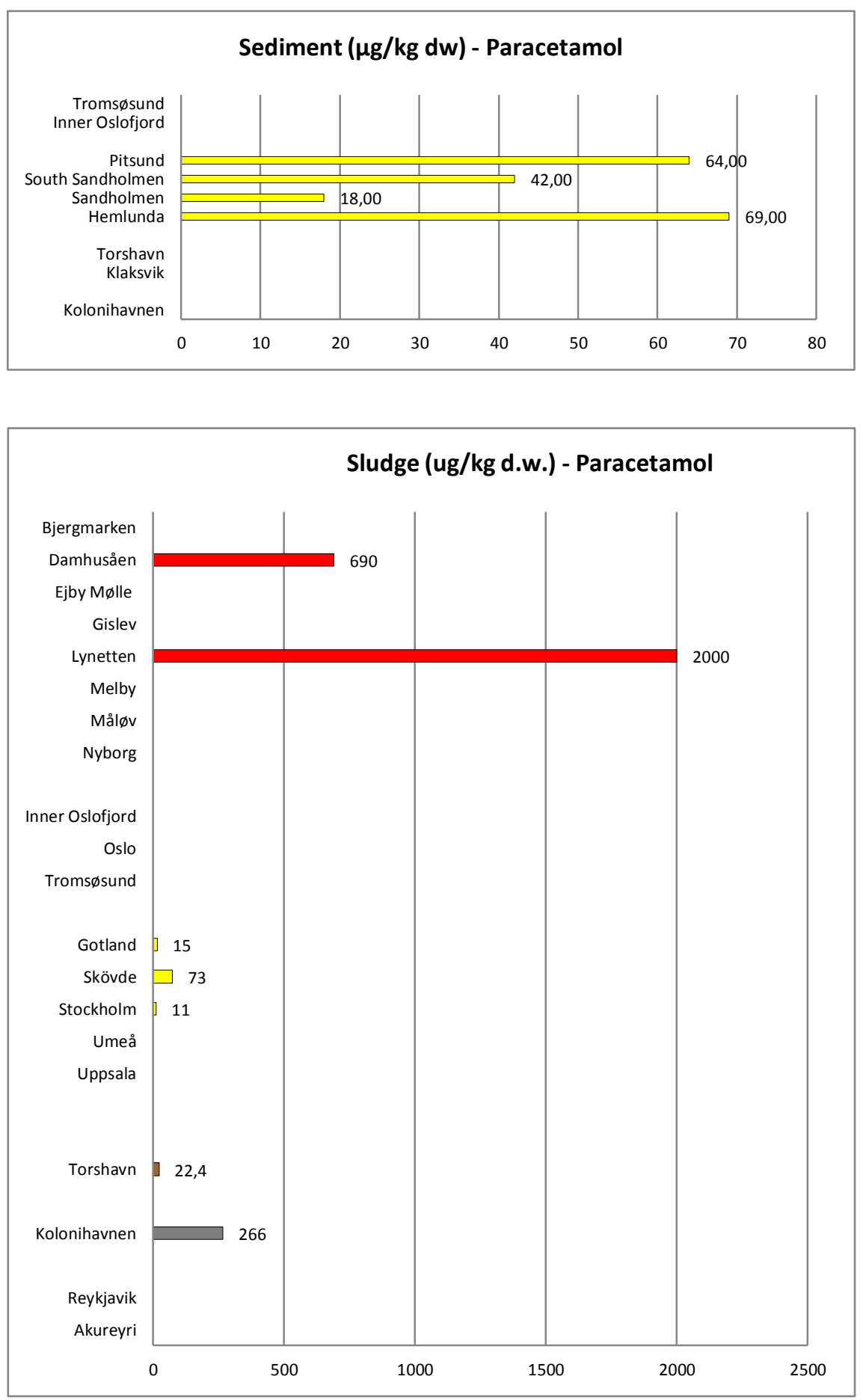


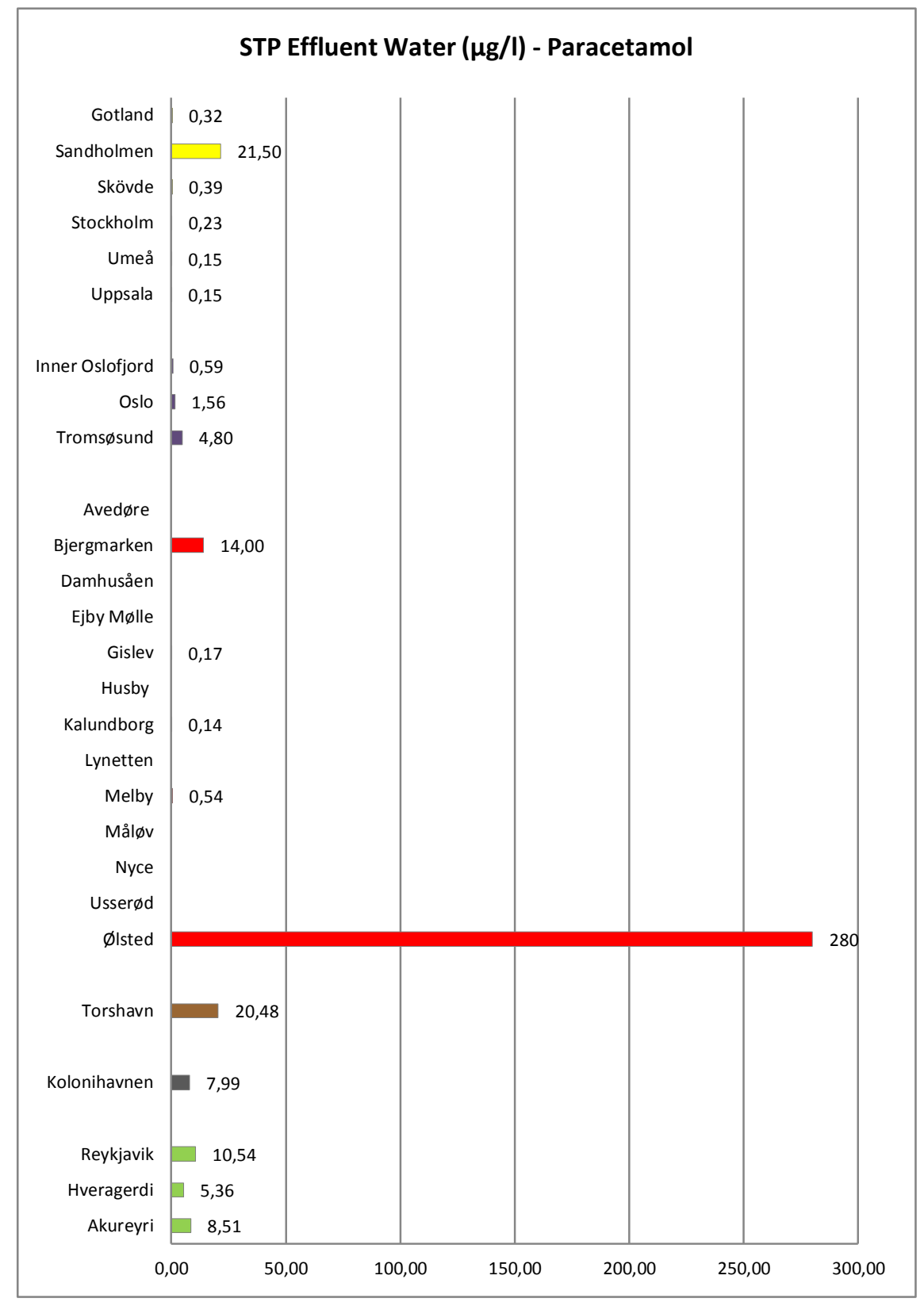




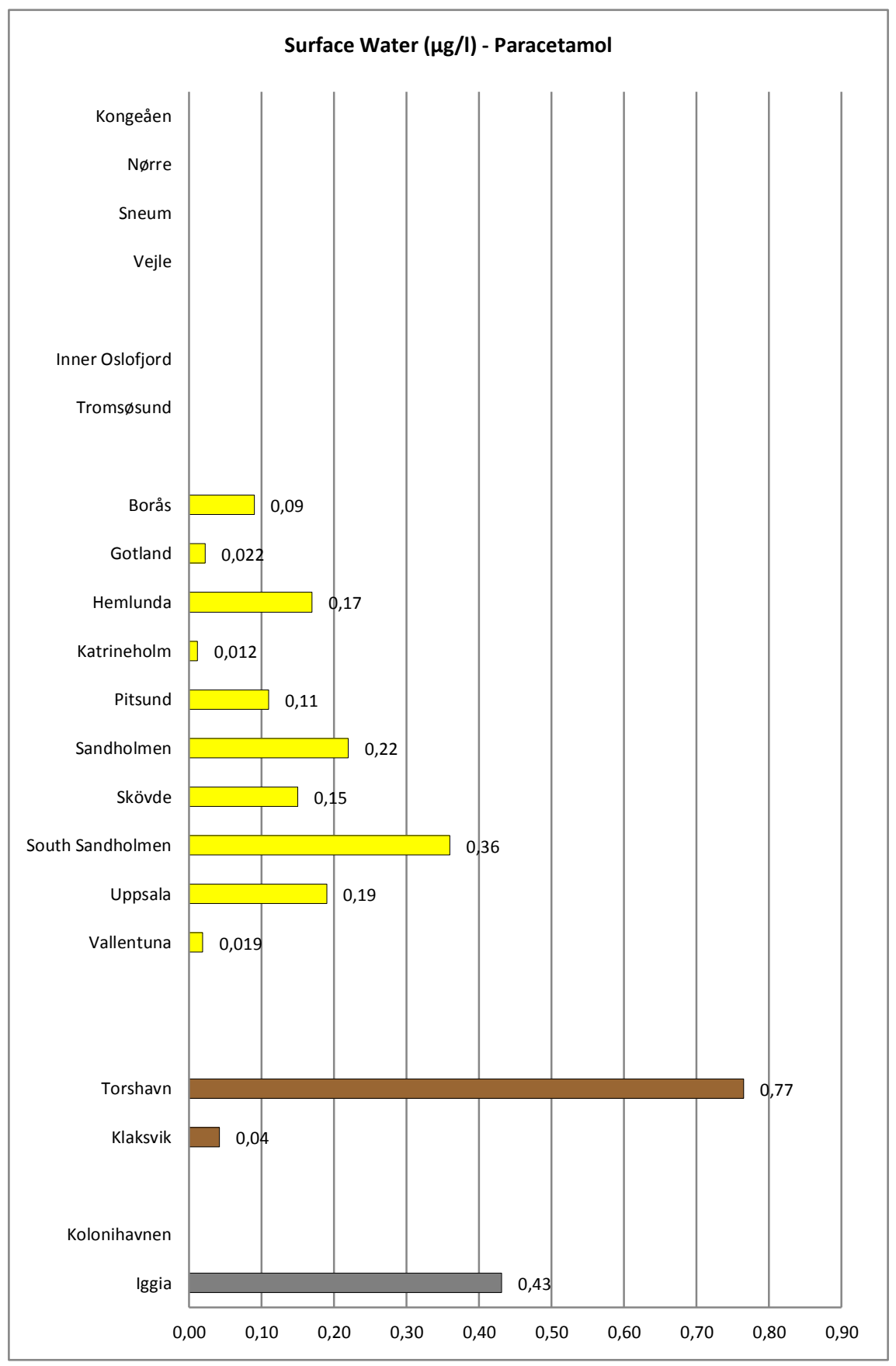




\begin{tabular}{|c|c|c|c|c|c|c|c|c|c|c|c|c|c|c|}
\hline \multirow{2}{*}{$\begin{array}{l}\text { Paracetamol } \\
\text { Matrix }\end{array}$} & \multicolumn{2}{|c|}{ Denmark } & \multicolumn{2}{|c|}{ Finland } & \multicolumn{2}{|c|}{ Norway } & \multicolumn{2}{|c|}{ Sweden } & \multicolumn{2}{|c|}{ Faroe Islands } & \multicolumn{2}{|c|}{ Greenland } & \multicolumn{2}{|c|}{ Iceland } \\
\hline & $\begin{array}{r}\operatorname{Min} \\
\max \\
A D\end{array}$ & $\begin{array}{l}\text { Nr.S } \\
\text { (ref) }\end{array}$ & $\begin{array}{r}\operatorname{Min} \\
\max \\
A D\end{array}$ & $\begin{array}{l}\text { Nr.S } \\
\text { (ref) }\end{array}$ & $\begin{array}{r}\min \\
\max \\
A D\end{array}$ & $\begin{array}{l}\mathrm{Nr} . \mathrm{S} \\
\text { (ref) }\end{array}$ & $\begin{array}{r}\min \\
\max \\
A D\end{array}$ & $\begin{array}{l}\text { Nr.S } \\
\text { (ref) }\end{array}$ & $\begin{array}{r}\min \\
\max \\
A D\end{array}$ & $\begin{array}{l}\text { Nr.S } \\
\text { (ref) }\end{array}$ & $\begin{array}{r}\min \\
\max \\
A D\end{array}$ & $\begin{array}{l}\text { Nr.S } \\
\text { (ref) }\end{array}$ & $\begin{array}{r}\min \\
\max \\
A D\end{array}$ & $\begin{array}{l}\mathrm{Nr} . \mathrm{S} \\
\text { (ref) }\end{array}$ \\
\hline $\begin{array}{l}\text { Biota } \\
(\mu \mathrm{g} / \mathrm{kg} \mathrm{ww})\end{array}$ & & & & & nd & 3 (c) & nd & 21 (ef) & & & & & & \\
\hline $\begin{array}{l}\text { Sediment } \\
(\mu \mathrm{g} / \mathrm{kg} \mathrm{dw})\end{array}$ & & & & & nd & $6(c)$ & $\begin{array}{r}18 \\
69 \\
48,25\end{array}$ & $4(f)$ & nd & $3(h)$ & nd & $2(h)$ & & \\
\hline $\begin{array}{l}\text { STP sludge } \\
(\mu \mathrm{g} / \mathrm{kg} \mathrm{dw})\end{array}$ & $\begin{array}{r}\text { nd } \\
2000 \\
1345\end{array}$ & 10 (a) & & & nd & $11(\mathrm{~cd})$ & $\begin{array}{l}\text { nd } \\
73 \\
33\end{array}$ & 5 (f) & $\begin{array}{r}\text { nd } \\
22,4 \\
22,4\end{array}$ & $3(h)$ & $\begin{array}{r}85,2 \\
447 \\
266\end{array}$ & $2(h)$ & nd & $3(h)$ \\
\hline $\begin{array}{l}\text { STP effluent } \\
(\mu \mathrm{g} / \mathrm{l})\end{array}$ & $\begin{array}{r}\text { nd } \\
280 \\
59,66\end{array}$ & $16(a b)$ & & & $\begin{array}{r}\text { nd } \\
6,01 \\
2,33\end{array}$ & $16(c d)$ & $\begin{array}{r}\text { nd } \\
29 \\
3,03\end{array}$ & 17 (ef) & $\begin{array}{l}20,5 \\
20,5 \\
20,5\end{array}$ & $1(\mathrm{~h})$ & $\begin{array}{l}8 \\
8 \\
8\end{array}$ & $1(\mathrm{~h})$ & $\begin{array}{r}3,66 \\
10,53 \\
7,65\end{array}$ & $5(h)$ \\
\hline $\begin{array}{l}\text { Surface water } \\
(\mu \mathrm{g} / \mathrm{l})\end{array}$ & nd & $16(a)$ & & & nd & $12(c)$ & $\begin{array}{r}\text { nd } \\
0,36 \\
0,1\end{array}$ & 23 (ef) & $\begin{array}{r}\text { nd } \\
0,93 \\
0,52\end{array}$ & $5(h)$ & $\begin{array}{l}\text { nd } \\
0,7 \\
0,4\end{array}$ & $5(h)$ & & \\
\hline Ground water & nd & 3 (a) & & & & & & & & & & & & \\
\hline $\begin{array}{l}\text { Drinking } \\
\text { water( } \mu \mathrm{g} / \mathrm{l})\end{array}$ & & & & & & & $\begin{array}{r}\text { nd } \\
0,015 \\
0,013\end{array}$ & $6(\mathrm{e})$ & & & & & & \\
\hline $\begin{array}{l}\text { STP Influent } \\
(\mu \mathrm{g} / \mathrm{l})\end{array}$ & $\begin{array}{r}140 \\
1200 \\
407,8\end{array}$ & 9 (a) & & & & & $\begin{array}{r}\text { nd } \\
540 \\
150,6\end{array}$ & 16 (ef) & $\begin{array}{r}26 \\
50,6 \\
38,3\end{array}$ & $2(h)$ & & & $\begin{array}{r}\text { nd } \\
11,2 \\
5,3\end{array}$ & $5(h)$ \\
\hline Pig slurry $(\mu \mathrm{g} / \mathrm{l})$ & $\begin{array}{r}\text { nd } \\
0,14 \\
0,14\end{array}$ & 4 (a) & & & & & & & & & & & & \\
\hline $\begin{array}{l}\text { Warning } \\
\text { system for } \\
\text { pesticide } \\
\text { leaching to } \\
\text { groundwater }\end{array}$ & nd & 8 (a) & & & & & & & & & & & & \\
\hline $\begin{array}{l}\text { Drain water } \\
(\mu \mathrm{g} / \mathrm{l})\end{array}$ & $\begin{array}{l}0,56 \\
0,56 \\
0,56\end{array}$ & 1 (a) & & & & & & & & & & & & \\
\hline $\begin{array}{l}\text { settleable } \\
\text { particulate } \\
\text { material } \\
(\mathrm{ng} / \mathrm{g} \mathrm{dw})\end{array}$ & & & $\begin{array}{r}\text { nd } \\
69,6 \\
34,8\end{array}$ & $11(\mathrm{~g})$ & & & & & & & & & & \\
\hline $\begin{array}{l}\text { Hospital WW } \\
(\mu \mathrm{g} / \mathrm{l})\end{array}$ & & & & & & & & & $\begin{array}{l}40,3 \\
71,5 \\
55,9\end{array}$ & $2(h)$ & $\begin{array}{l}20,6 \\
25,8 \\
23,2\end{array}$ & $1(\mathrm{~h})$ & & \\
\hline
\end{tabular}

\section{References:}
a) (Mogensen, et al., 2008)
b) (Kjølholt, et al., 2003)
c) (Schlabach, et al., 2009)
d) (Thomas, et al., 2007)
e) (Fick, et al., 2011)
f) (Remberger, et al., 2009) 
g) (Lahti \& Oikari, 2011)

h) (Huber, et al., NA)

\section{Conclusion:}

The compound has been monitored in one study in Finland, two in Denmark, Norway and Swede, and one international study covering Faroe Islands, Greenland and Iceland. The compound has been detected in several matrices:

- Sediment in Sweden, but not in Norway, Faroe Islands and Greenland

- Surface water in Sweden, Faroe Islands, and Greenland, not Norway and Denmark

- STP sludge in Denmark, Sweden, Faroe Islands and Greenland, not in Norway and Iceland. The values detected in Denmark were significantly higher than in the other countries

- STP effluent water, in Denmark, Norway, Sweden, Faroe Islands and Iceland. However the values detected in Denmark were much higher than in the other countries

- Drinking water in Sweden

- STP influent water in Denmark, Sweden, Faroe Islands and Iceland, in higher concentrations in Denmark

- Hospital waste water in Faroe Islands and Greenland

- Pig slurry in Denmark 


\subsection{Compounds monitored in at least two studies}

\subsubsection{Fentanyl}

CAS nr.: 437-38-7

Info: Potent synthetic narcotic analgesic

Toxicity data: NA

$P N E C=\mathrm{NA}$

\begin{tabular}{|c|c|c|}
\hline Fentanil & Sweder & \\
\hline Matrix & $\begin{array}{r}\text { Min } \\
\max \\
A D\end{array}$ & $\begin{array}{l}\text { Nr.S } \\
\text { (ref) }\end{array}$ \\
\hline Biota $(\mu \mathrm{g} / \mathrm{kg} w \mathrm{w})$ & nd & $24(a b)$ \\
\hline Sediment & nd & 9 (a) \\
\hline STP effluent $(\mu \mathrm{g} / \mathrm{l})$ & $\begin{array}{l}\text { nd } \\
3,7 \\
2,4\end{array}$ & $13(b)$ \\
\hline STP sludge $(\mu \mathrm{g} / \mathrm{kg} \mathrm{dw})$ & $\begin{array}{r}\text { nd } \\
0,79 \\
0,75\end{array}$ & $40(a b)$ \\
\hline Surface water $(\mu \mathrm{g} / \mathrm{l})$ & $\begin{array}{r}\text { nd } \\
0,004 \\
0,002\end{array}$ & $33(a b)$ \\
\hline STP Influent $(\mu \mathrm{g} / \mathrm{l})$ & $\begin{array}{r}\text { nd } \\
0,008 \\
0,002\end{array}$ & $67(a b)$ \\
\hline Drinking water & nd & 6 (b) \\
\hline Hospital WW & nd & 2 (a) \\
\hline
\end{tabular}

\section{References:}

a) (Woldegiorgis, et al., 2007b)

b) (Fick, et al., 2011)

\section{Conclusion:}

The compound has only been monitored in Sweden and was detected in surface water, STP sludge, influent and effluent. 


\subsection{Compounds monitored in one study}

\begin{tabular}{lcc}
\hline & Dextropropoxyphen (469-62-5) & Nr.S \\
\hline Matrix & $\operatorname{Min}$ & AD \\
& nd & 9 \\
Biota (ng/g ww) & nd & 9 \\
Sediment (ng/g dw) & nd & 35 \\
STP sludge (ng/g dw) & 1 & \\
& 1 & nd \\
STP effluent and influent (ng/l) & nd & 19 \\
Surface water (ng/l) & nd & 5 \\
Landfill leachates (ng/l) & nd & 2 \\
Hospital WW (ng/l) & & 59 \\
\hline
\end{tabular}

\section{Reference:}

- (Woldegiorgis, et al., 2007b)

\begin{tabular}{|c|c|c|c|c|}
\hline & $\begin{array}{r}\text { Buprenorphine } \\
(52485-79-7)\end{array}$ & $\begin{array}{r}\text { Codeine } \\
(76-57-3)\end{array}$ & $\begin{array}{r}\text { Tramadol } \\
(46941-76-8 / \\
27203-92-5)\end{array}$ & \\
\hline Matrix & $\begin{array}{r}\operatorname{Min} \\
\max \\
A D\end{array}$ & $\begin{array}{r}\operatorname{Min} \\
\max \\
A D\end{array}$ & $\begin{array}{r}\operatorname{Min} \\
\max \\
A D\end{array}$ & Nr.S \\
\hline Biota $(\mu \mathrm{g} / \mathrm{kg})$ & $\begin{array}{l}\text { nd } \\
17 \\
17\end{array}$ & $\begin{array}{r}\text { nd } \\
0,84 \\
0,84\end{array}$ & nd & 15 \\
\hline Sludge ( $\mu \mathrm{g} / \mathrm{kg} \mathrm{dw}$ ) & $\begin{array}{r}21 \\
140 \\
63,4\end{array}$ & $\begin{array}{r}9,5 \\
29 \\
17,7\end{array}$ & $\begin{array}{r}\text { nd } \\
68 \\
62,5\end{array}$ & 5 \\
\hline Effluent (ng/l) & $\begin{array}{r}10 \\
64 \\
27,1\end{array}$ & $\begin{array}{r}78 \\
780 \\
358\end{array}$ & $\begin{array}{r}300 \\
3000 \\
1687\end{array}$ & 13 \\
\hline Surface water (ng/l) & $\begin{array}{r}n d \\
33 \\
25,7\end{array}$ & $\begin{array}{r}\text { nd } \\
340 \\
59,9\end{array}$ & $\begin{array}{r}\text { nd } \\
1800 \\
376\end{array}$ & 15 \\
\hline STP influent (ng/l) & $\begin{array}{r}31 \\
1000 \\
239\end{array}$ & $\begin{array}{r}390 \\
4200 \\
1242\end{array}$ & $\begin{array}{r}770 \\
6100 \\
1972\end{array}$ & 12 \\
\hline Drinking water (ng/l) & $\begin{array}{l}\text { nd } \\
16 \\
16\end{array}$ & nd & nd & 6 \\
\hline
\end{tabular}

\section{Reference:}

- (Fick, et al., 2011) 


\begin{tabular}{lcc}
\hline & Morphine (57-27-2) & Nr.S \\
\hline Matrix & $\operatorname{Min}$ & $\max$ \\
& AD & 3 \\
& nd & 6 \\
Biota & nd & 4 \\
Sediment & nd & 10 \\
STP sludge (ng/g dw) & nd & \\
STP effluent (ng/l) & 980 & 12 \\
& 604,7 & \\
Surface water (ng/l) & nd & \\
& 21,7 & 13,8 \\
\hline
\end{tabular}

\section{Reference:}

- (Schlabach, et al., 2009) 


\section{Antibiotics, antiseptics, germicides and anti-fungals}

\subsection{Scope and definition}

Antibiotics are defined as the subgroup of anti-infectives that are derived from bacterial sources and are used to treat bacterial infections. Other classes of drugs, most notably the sulfonamides, are an effective antibacterial that will also be included as antibiotic. (Internal and external use).

Antiseptics and germicides are chemical agents that destroy microorganisms that cause disease. Antiseptics are applied to the skin or mucus membranes to cleanse wounds and prevent infections. (External use).

A microorganism or microbe is a microscopic organism that comprises either a single cell (unicellular), cell clusters, or no cell at all (acellular).

Antifungal is a drug used to treat fungal infections (external and internal use). Anti-fungals used in human or veterinary treatment are included in this study. Fungicides for other agricultural uses are not included.

Fungus is defined as a single-celled or multicellular organism. Fungi can be true pathogens (such as histoplasmosis and coccidioidomycosis) that cause infections in healthy persons or they can be opportunistic pathogens (such as aspergillosis, candidiasis, and cryptococcosis) that cause infections in immune-compromised persons.

The PPCP category "Antibiotics, antiseptics, germicides and antifungals" does not include: disinfectants, detergents that may have antimicrobial effects, other anti-parasitics (e.g. intestinal or heart worm infection, malaria, etc.) and pesticides.

Disinfectant is defined as an agent freeing from infection or infectionproducing organisms and applied to inanimate objects, as they are typically too strong to be used on living tissues.

The environmental risks related to the emission of these compounds in the environment is expected to vary from one compound to another, as their ability to persist, bioaccumute and their intrinsic toxicity will be different from one compound to the other. However, the primary risk related to the increased use and thereby emission of these compounds and in particular antibiotics, is the risk of developing antibiotic resistant bacteria in the environment. Resistance can be disseminated to pathogenic bacteria, which is an important threat to public health. In addition, changing the natural composition of micro-organisms in the environment may also have effects on the whole ecosystem. 
Table 2 presents the antibiotics, antiseptic, germicides and anti-fungal identified in monitoring studies in the Nordic countries.

Table2: Antibiotics, antiseptic, germicides and anti-fungal identified in monitoring studies in the Nordic countries

\begin{tabular}{|c|c|}
\hline Compounds monitored & CAS nr \\
\hline 1,2,3,4-Tetrachlorobenzene (antimicrobial and antifungal activity) & $634-66-2$ \\
\hline 1,2,3,5-Tetrachlorobenzene (antimicrobial and antifungal activity) & $634-90-2$ \\
\hline $1,2,4,5-$ Tetrachlorobenzene (antimicrobial and antifungal activity) & $95-94-3$ \\
\hline 1,2,3-Trichlorobenzene (antimicrobial and antifungal activity) & $87-61-6$ \\
\hline 1,2,4-Trichlorobenzene (antimicrobial and antifungal activity) & $120-82-1$ \\
\hline 1,3,5-Trichlorobenzene (antimicrobial and antifungal activity) & $108-70-3$ \\
\hline 2(Tiocyanomethylthio)benzothiazole (biocide) & $21564-17-0$ \\
\hline 4-Chloro-3-cresol (antiseptic, desinfectans, and fungicide) & $59-50-7$ \\
\hline 4-chloro-3-methylphenol (antiseptic) & $59-50-7$ \\
\hline Amoxicillin (antibiotic) & 26787-78-0 \\
\hline Ampicillin (antibiotic) & $69-53-4$ \\
\hline ATAC-C16: Cetrimonium salts (antispetic) & $112-02-7 / 57-09-0$ \\
\hline Azithromycin (antibiotic) & $83905-01-5$ \\
\hline Benzyl penicillin (antibiotic) & $61-33-6$ \\
\hline Bronopol (antimicrobial, preservative) & $52-51-7$ \\
\hline Cefadroxil (antibiotic) & $66592-87-8$ \\
\hline Cefalotin(antibiotic) & $153-61-7$ \\
\hline Cefotaxime (antibiotic) & $63527-52-6$ \\
\hline Cefuroxime & $55268-75-2$ \\
\hline Chlorhexidine (antiseptic) & $55-56-1$ \\
\hline Chlorocycline & 82-93-9 \\
\hline Chlortetracycline (antibiotic) & $57-62-5$ \\
\hline Ciprofloxacin (antibiotic) & 85721-33-1 \\
\hline Clarithromycin (antibiotic) & $81103-11-9$ \\
\hline Clindamycin (antibiotic) & $18323-44-9$ \\
\hline Danofloxacin (antibiotic) & $112398-08-0$ \\
\hline DDMAC & $7173-51-5$ \\
\hline Demeclocycline (antibiotic) & $127-33-3$ \\
\hline Difloxacillin (antibiotic) & $3116-76-5$ \\
\hline Dihydrostreptomycin (antibiotic) & $128-46-1$ \\
\hline Doxycycline (antibiotic) & $564-25-0$ \\
\hline Enrofloxacin (antibiotic) & $93106-60-6$ \\
\hline Erythromycin (antibiotic) & $114-07-8$ \\
\hline Florfenicol (antibiotic) & $73231-34-2$ \\
\hline Fluconazole (Antifungal drug) & $86386-73-4$ \\
\hline Flumequine (antibiotic) & $42835-25-6$ \\
\hline Gentamycin (antibiotic) & $1403-66-3$ \\
\hline Ketoconazol (antifungal) & $65277-42-1$ \\
\hline Linezolid (antibacterial agent) & $165800-03-3$ \\
\hline Marbofloxacin (antibiotic) & $115550-35-1$ \\
\hline Meclocycline (antibiotic) & $2013-58-3$ \\
\hline Meropenem (antibiotic) & $119478-56-7$ \\
\hline Metronidazol (antibiotic) & $443-48-1$ \\
\hline Miconazol (antifungal agent) & $22916-47-8$ \\
\hline Norfloxacin (antibiotic) & $70458-96-7$ \\
\hline Ofloxacin (antibiotic) & $82419-36-1$ \\
\hline Oxolinic acid (antibiotic) & $14698-29-4$ \\
\hline Oxytetracycline (antibiotic) & $79-57-2$ \\
\hline Penicillin V & $87-08-1$ \\
\hline Phenoxymethylpenicillin (antibiotic) & $87-08-1$ \\
\hline Pivmecillinam (antibiotic) & $33817-20-8$ \\
\hline Propiconazole (fungicide) & $60207-90-1$ \\
\hline Resorcinol (antiseptic and disinfectant) & $108-46-3$ \\
\hline Roxithromycin (antibiotic) & $80214-83-1$ \\
\hline Sarafloxacin (antibiotic) & $98105-99-8$ \\
\hline \multicolumn{2}{|l|}{ Silver (germicide) } \\
\hline Spiramycin (antibiotic) & $8025-81-8$ \\
\hline Sulfabenzamide (antibacterial/antimicrobial) & $127-71-9$ \\
\hline Sulfacetamide (antibiotic) & $144-80-9$ \\
\hline Sulfachloropyridazine (Antibacterial) & $80-32-0$ \\
\hline Sulfaclozin (antibiotic) & $102-65-8$ \\
\hline Sulfadiazine (antibiotic) & $68-35-9$ \\
\hline
\end{tabular}




\begin{tabular}{ll}
\hline Compounds monitored & CAS nr \\
\hline Sulfadimethoxine (antibiotic) & $122-11-2$ \\
Sulfadimidine(antibiotic) & $57-68-1$ \\
Sulfadoxine (Anti-Infective Agents also used in Antimalarials) & $2447-57-6$ \\
Sulfaguanidine(antibiotic) & $57-67-0$ \\
Sulfamerazine (sulfonamide anitbacterial) & $127-79-7$ \\
Sulfameter (sulfonamide antibacerial) & $651-06-9$ \\
Sulfamethizol (antibiotic) & $144-82-1$ \\
Sulfamethoxazole (antibiotic) & $723-46-6$ \\
Sulfamethoxypyridazine (sulfonamide antibacterial) & $000080-35-3$ \\
Sulfamonomethoxine (antibiotic) & $1220-83-3$ \\
Sulfamoxole (sulfonamide antibacterial) & $729-99-7$ \\
Sulfanilamide (sulfonamide antibacterial) & $63-74-1$ \\
Sulfapyridine (sulfonamide antibacterial) & $000144-83-2$ \\
Sulfaquinoxaline (Coccidia treatment, intestinal unicellular parasite) & $59-40-5$ \\
Sulfathiazol (antimicrobial) & $72-14-0$ \\
Sulfatroxazole (sulfonamide antibacterial) & $23256-23-7$ \\
Sulphafurazole (antibiotic) & $127-69-5$ \\
Tetracycline (antibiotic) & $60-54-8$ \\
Tiamulin (antibiotic) & $55297-95-5$ \\
Triclosan & $3380-34-5$ \\
Trimethoprim (antibiotic) & $738-70-5$ \\
Tylosin ((antibiotic) & $1401-69-0$ \\
Zinc pyrithione (ZPT) (antimicrobial to fungi and bacteria, used in particular to & $13463-41-7$ \\
combat dandruf fungi) & \\
\hline
\end{tabular}

\subsection{Compounds mapped}

\subsubsection{Ciprofloxacin}

CAS nr.: 85721-33-1

Info: Synthetic chemotherapeutic antibiotic of the fluoroquinolone drug class

Toxicity data: NA

$P N E C=N A$

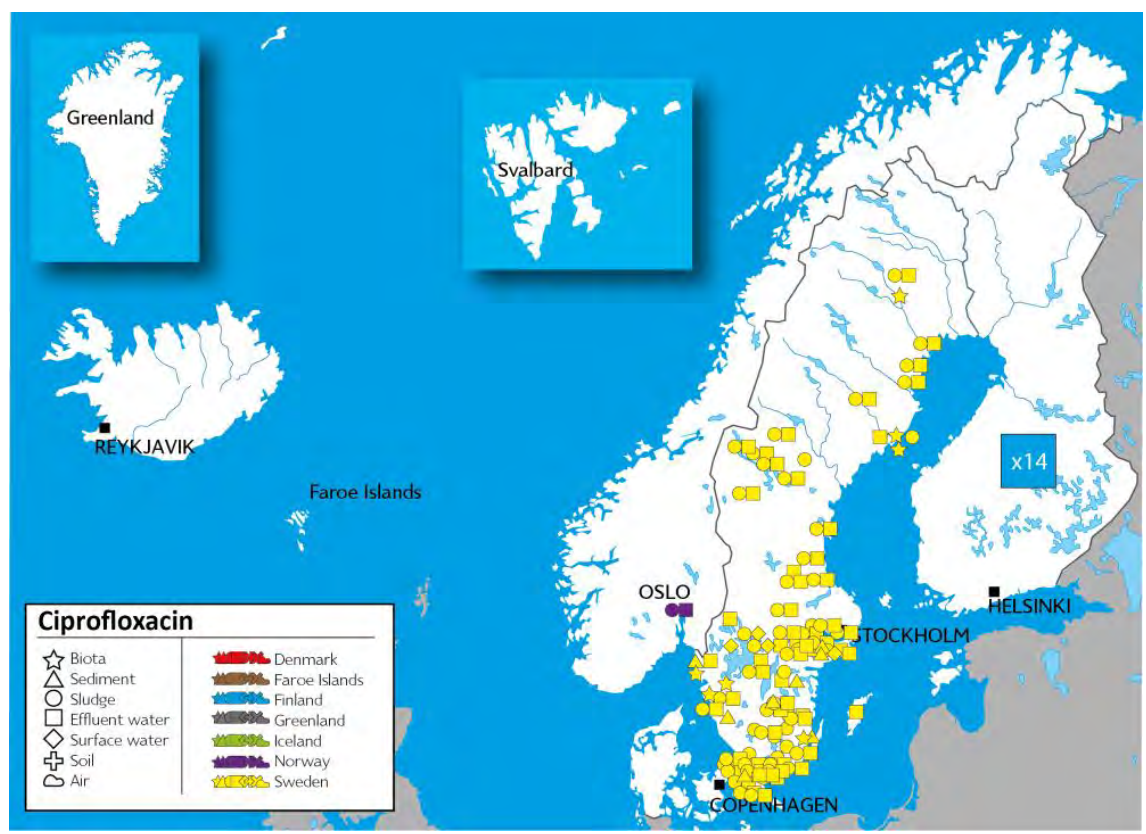



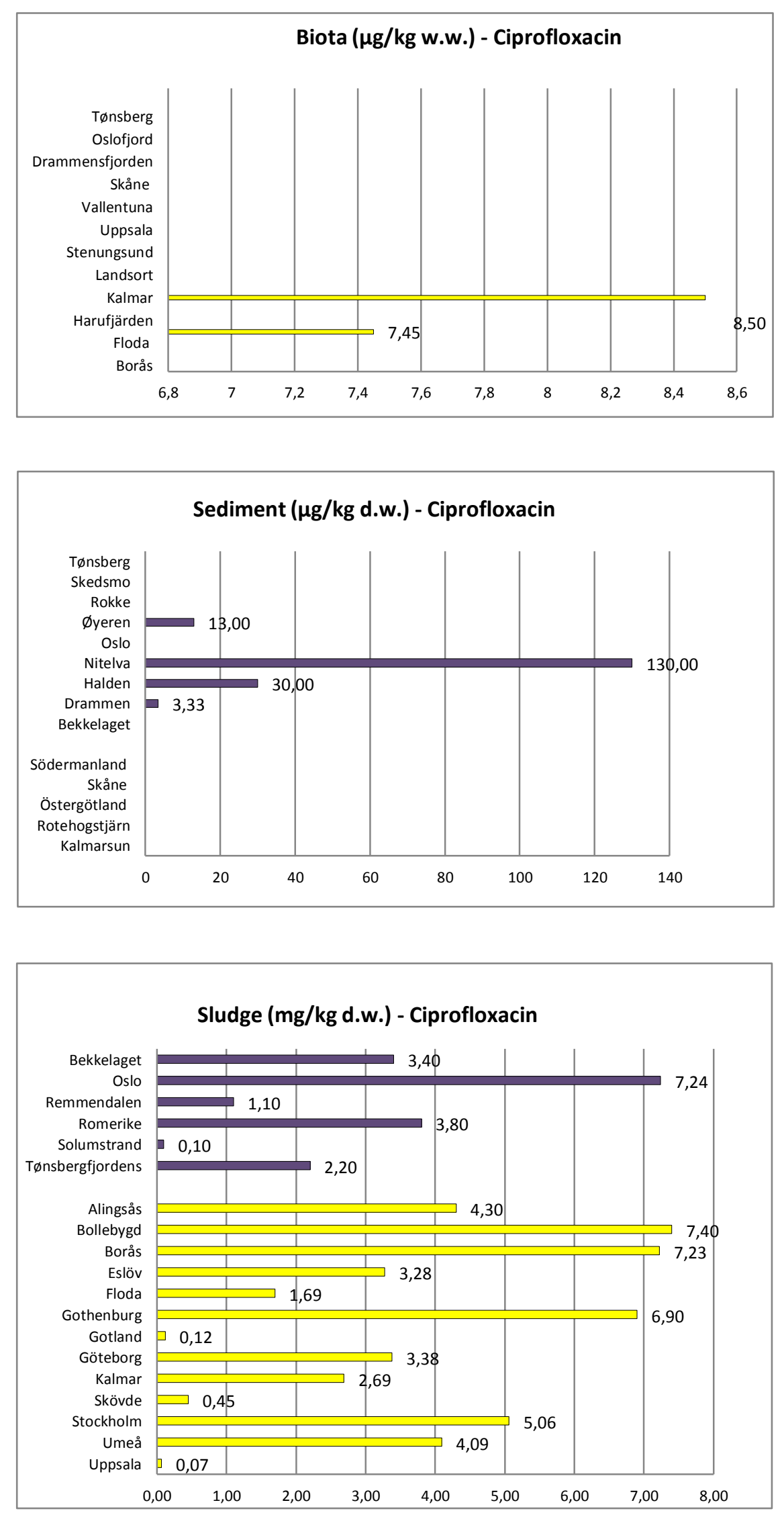

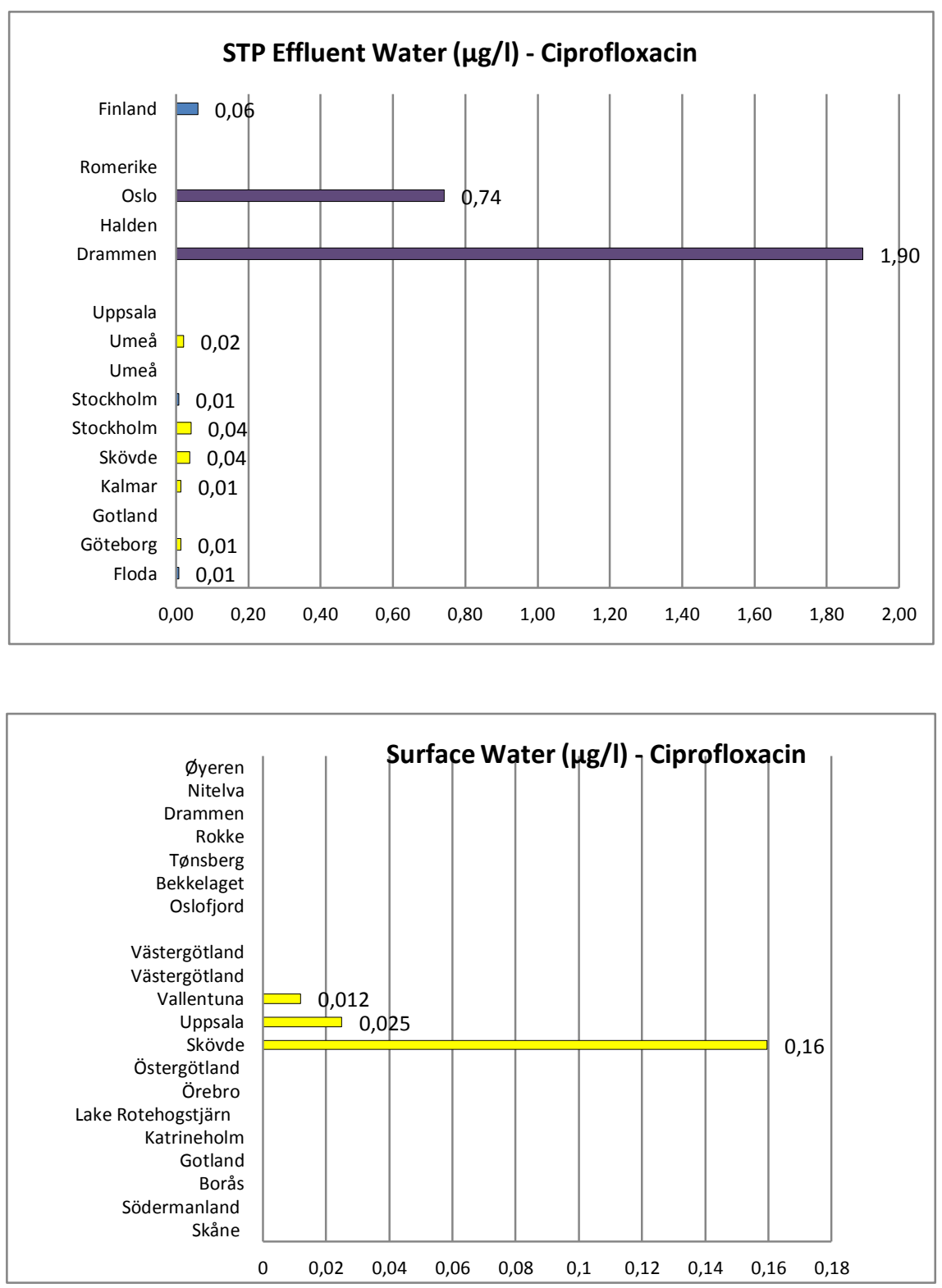

\begin{tabular}{|c|c|c|c|c|c|c|}
\hline $\begin{array}{l}\text { Ciprofloxacin } \\
\text { Matrix }\end{array}$ & \multicolumn{2}{|c|}{ Finland } & \multicolumn{2}{|c|}{ Norway } & \multicolumn{2}{|c|}{ Sweden } \\
\hline & $\begin{array}{r}\text { Min } \\
\max \\
A D\end{array}$ & $\begin{array}{l}\text { Nr.S } \\
\text { (ref) }\end{array}$ & $\begin{array}{r}\text { Min } \\
\max \\
A D\end{array}$ & $\begin{array}{l}\text { Nr.S } \\
\text { (ref) }\end{array}$ & $\begin{array}{r}\operatorname{Min} \\
\max \\
\text { AD }\end{array}$ & $\begin{array}{l}\mathrm{Nr} . \mathrm{S} \\
\text { (ref) }\end{array}$ \\
\hline Biota $(\mu \mathrm{g} / \mathrm{kg} \mathrm{ww})$ & & & nd & 5 (b) & $\begin{array}{r}\text { nd } \\
8,5 \\
7,97\end{array}$ & 15 (de) \\
\hline Sediment $(\mu \mathrm{g} / \mathrm{kg} \mathrm{dw})$ & & & $\begin{array}{r}\text { nd } \\
130 \\
44,1\end{array}$ & 9 (b) & nd & $5(d f)$ \\
\hline STP effluent $(\mu \mathrm{g} / \mathrm{l})$ & $\begin{array}{r}\text { NA } \\
\text { NA } \\
0,06\end{array}$ & 14 (a) & $\begin{array}{r}\mathrm{nd} \\
1,9 \\
1,32\end{array}$ & 4 (bc) & $\begin{array}{r}\text { nd } \\
0,041 \\
0,02\end{array}$ & 10 (de) \\
\hline
\end{tabular}




\begin{tabular}{|c|c|c|c|c|c|c|}
\hline \multirow{2}{*}{$\begin{array}{l}\text { Ciprofloxacin } \\
\text { STP sludge }(\mathrm{mg} / \mathrm{kg} \mathrm{dw})\end{array}$} & \multicolumn{2}{|c|}{ Finland } & \multicolumn{2}{|c|}{ Norway } & \multicolumn{2}{|c|}{ Sweden } \\
\hline & & & 0,10 & $13(\mathrm{bc})$ & 0,07 & 41 (degh) \\
\hline & & & 7,24 & & 7,40 & \\
\hline & & & 2,97 & & 3,6 & \\
\hline \multirow[t]{3}{*}{ Surface water( $\mu \mathrm{g} / \mathrm{l})$} & & & & \multirow[t]{3}{*}{7 (b) } & nd & \multirow[t]{3}{*}{13 (de) } \\
\hline & & & \multirow{2}{*}{ nd } & & 0,16 & \\
\hline & & & & & 0,066 & \\
\hline \multicolumn{3}{|l|}{ Groundwater } & & & nd & $2(f)$ \\
\hline \multicolumn{3}{|l|}{ Manure } & & & nd & $6(f)$ \\
\hline \multicolumn{3}{|l|}{ Soil from farms } & & & nd & $6(f)$ \\
\hline \multirow[t]{3}{*}{ STP Influent $(\mu \mathrm{g} / \mathrm{l})$} & NA & $14(\mathrm{a})$ & nd & \multirow{3}{*}{8 (b) } & 0,09 & \multirow{3}{*}{$5(d$} \\
\hline & NA & & 5,4 & & 0,194 & \\
\hline & 0,6 & & 2,92 & & 0,133 & \\
\hline \multirow[t]{3}{*}{ Hospital WW $(\mu \mathrm{g} / \mathrm{l})$} & & & & & 2,4 & \multirow{3}{*}{2 (de) } \\
\hline & & & & & 101 & \\
\hline & & & & & NA & \\
\hline \multicolumn{3}{|l|}{ Drinking water } & & & $\mathrm{Nd}$ & 6 (de) \\
\hline \multirow[t]{3}{*}{ Untreated landfill leachates $\mu \mathrm{g} / \mathrm{l}$} & & & nd & \multirow{3}{*}{4 (b) } & & \\
\hline & & & 0,69 & & & \\
\hline & & & 0,69 & & & \\
\hline Treated landfill leachates $(\mu \mathrm{g} / \mathrm{l})$ & & & nd & 5 (b) & & \\
\hline Landfill leachate sediment $(\mu \mathrm{g} / \mathrm{kg} \mathrm{dw})$ & & & nd & $4(b)$ & & \\
\hline
\end{tabular}

\section{References:}

a) (Vieno, 2008)

b) (Møskeland, et al., 2006)

c) (Thomas, et al., 2007)

d) (Johansson, et al., 2003)

e) (Fick, et al., 2011)

f) (Sternbeck, et al., 2007a)

g) (Haglund \& Olofsson, 2006)

h) (Haglund \& Olofsson, 2007)

\section{Conclusion:}

The compound was monitored in 14 STPs in Finland, two studies in the South East of Norway and five studies throughout Sweden. The compound was detected in several matrices:

- Biota in two locations in Sweden, not in Norway

- Sediment in approx. 50\% of all samples in Norway, not in Sweden

- STP effluent water in all three countries, however the values detected in Norway were approx. 20 times higher than those observed in Finland and Sweden

- STP sludge in all samples in Sweden and Norway. There were important variations in the values detected within each country 
- Surface water in three locations in Sweden, the concentration was about ten times higher in one location. The compound was not detected in surface water in Norway

- In one untreated landfill leachate sample in Norway

- STP influent in all three countries

- Hospital waste water in Sweden

\subsubsection{Doxycycline}

CAS nr.: 564-25-0

Info: Tetracycline antibiotic

Toxicity data:

LOEC 7 days (Lemna gibba), growth $=300 \mu \mathrm{g} / \mathrm{l}$

LC50 14 days (Eisenia fotida) $>1000 \mathrm{mg} / \mathrm{l}$

LC50 $48 \mathrm{hr}$ (Microcystis aeruginosa) $=0,0037 \mathrm{mg} / \mathrm{l}$

LC50 $48 \mathrm{hr}$ (Daphnia magna) $=156,4 \mathrm{mg} / \mathrm{l}$

EC50 $24 \mathrm{hr}$ (Vibrio fischeri) $=163 \mathrm{mg} / \mathrm{l}$

(Hellström \& Kreuger, 2005)

$P N E C=\mathrm{NA}$

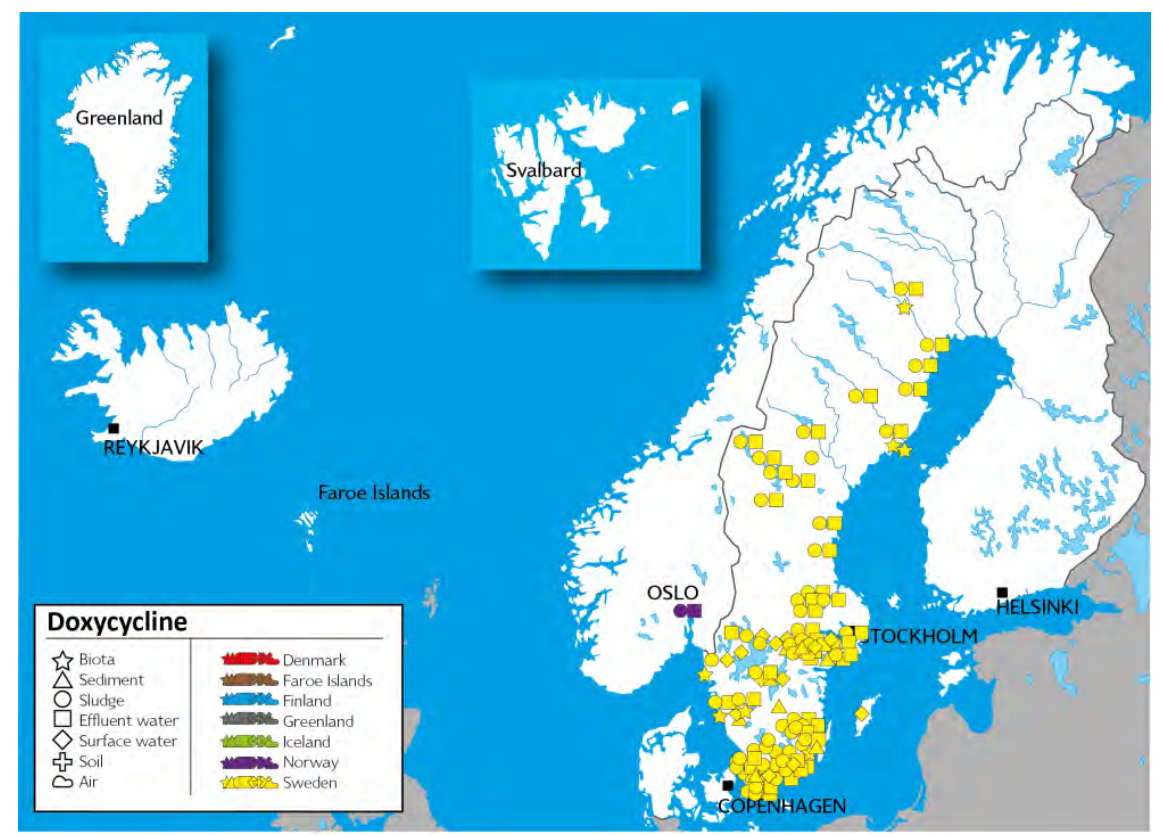




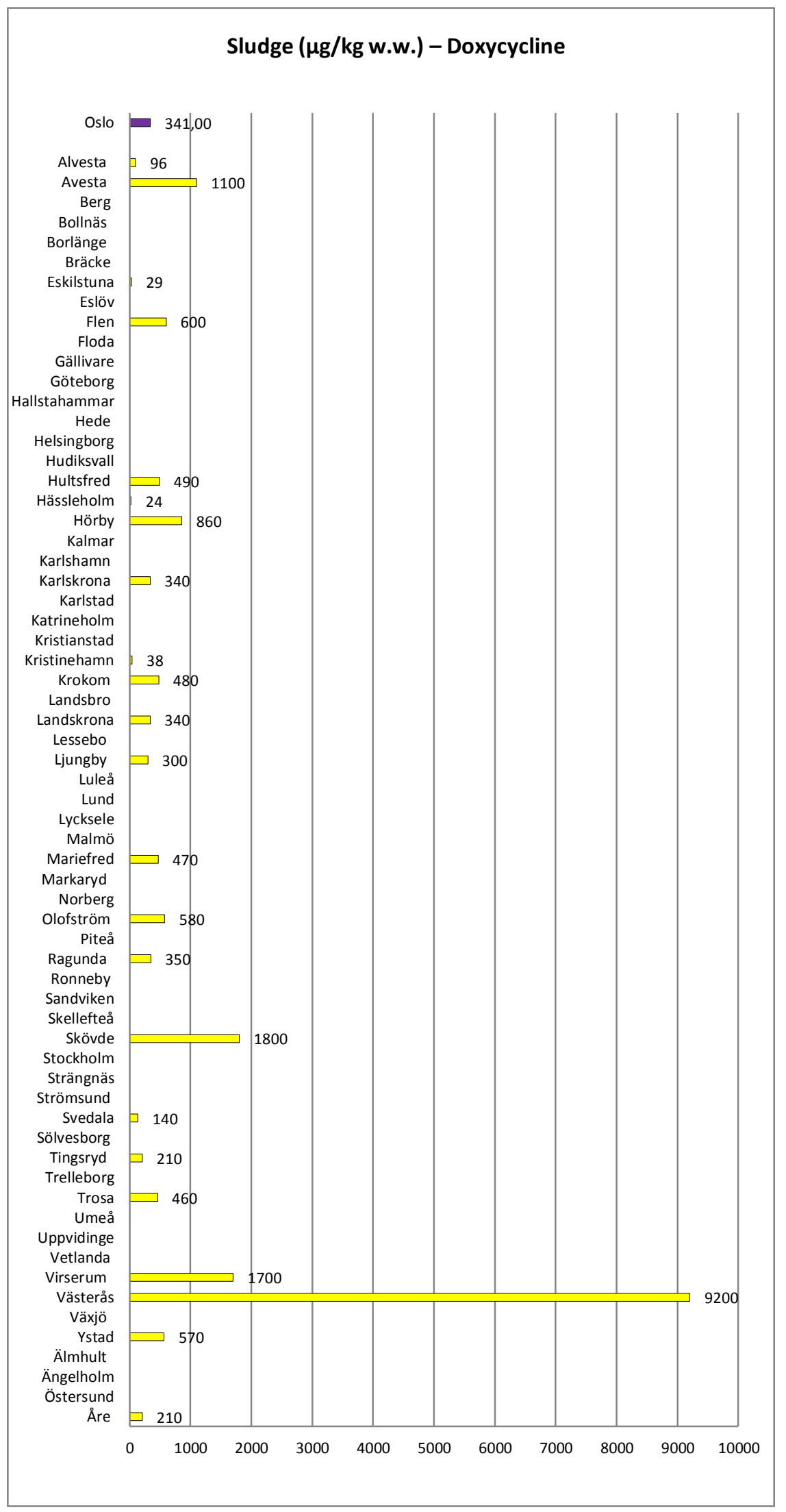




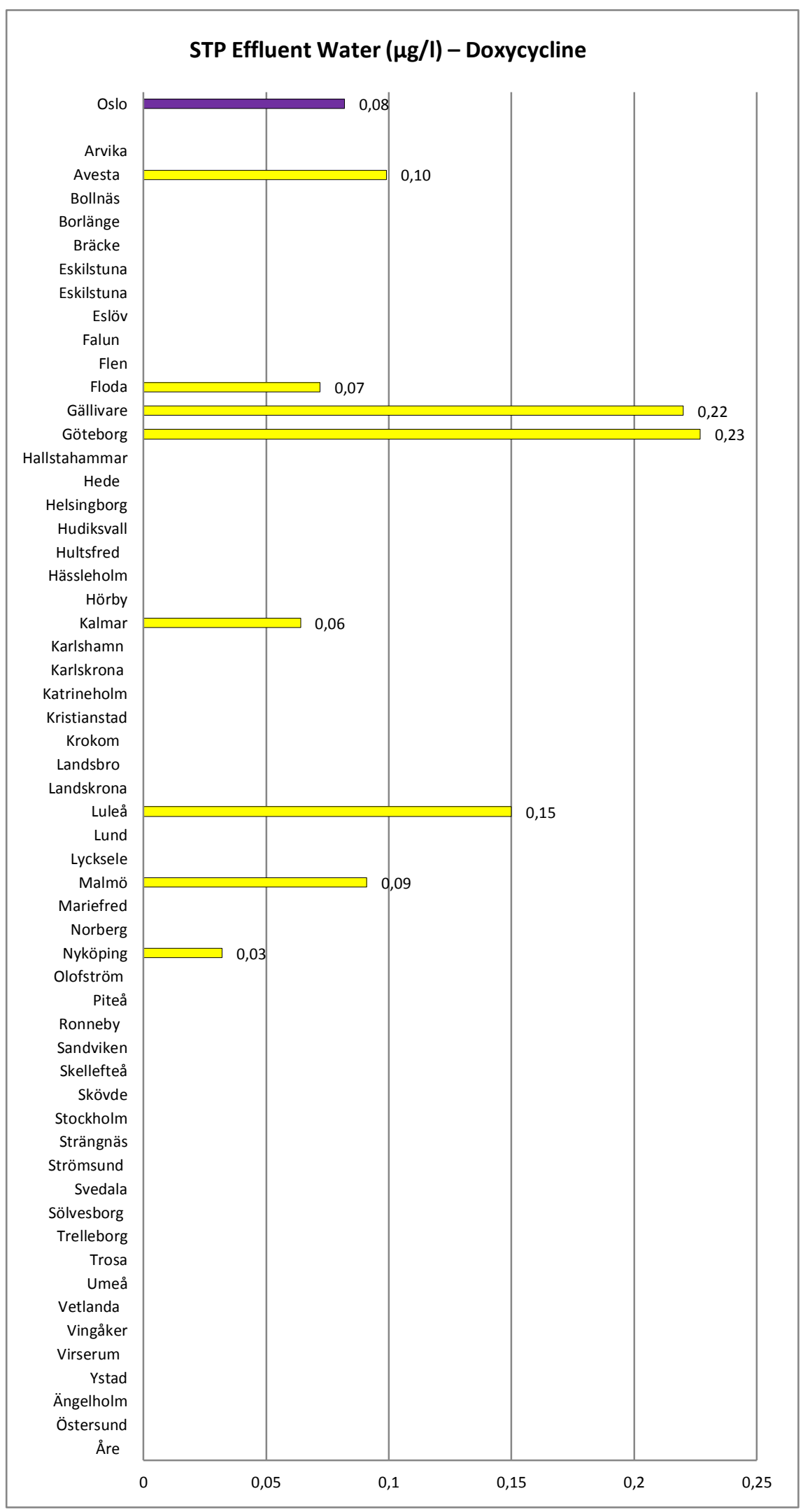




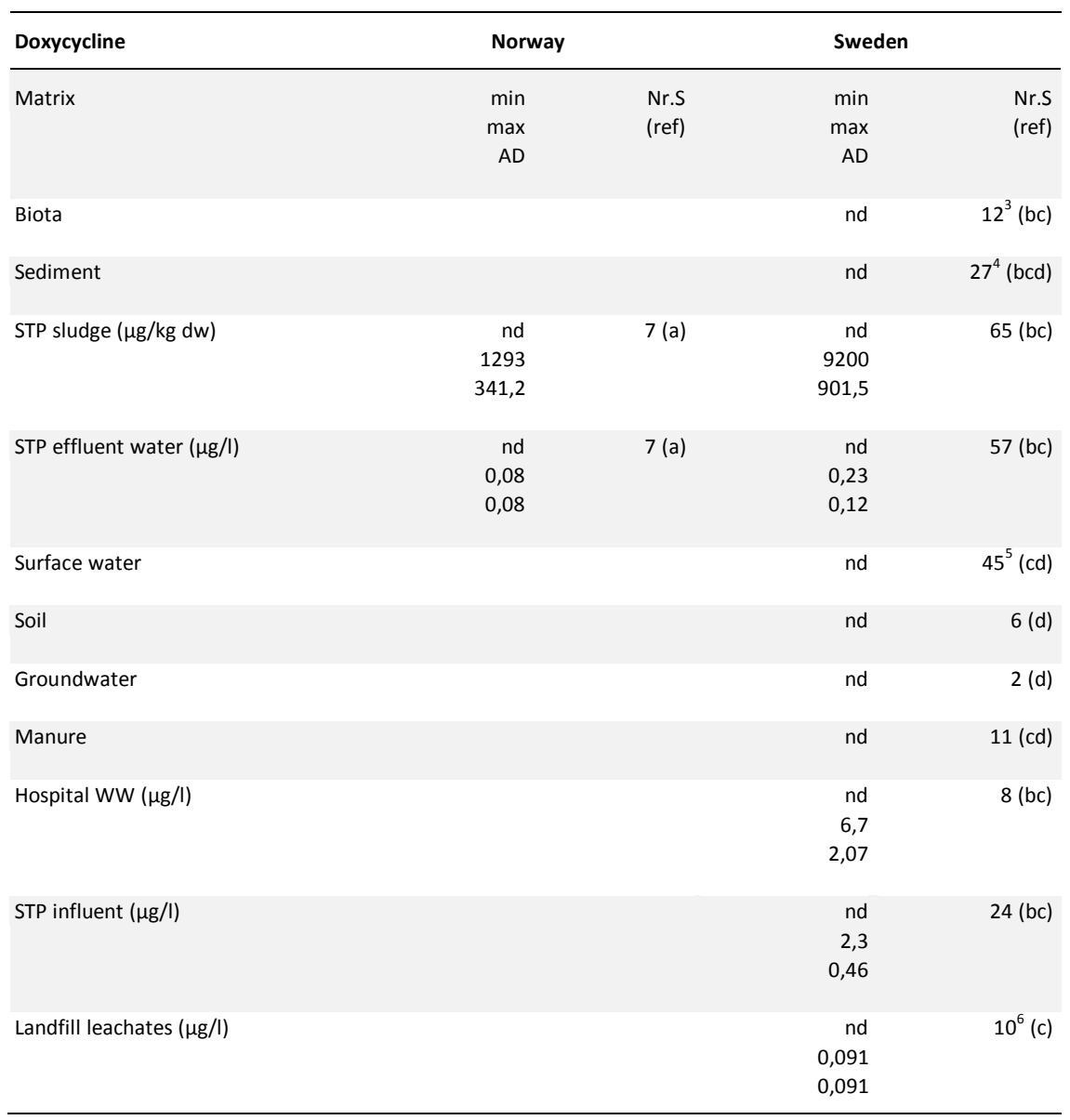

\section{References:}

a) (Thomas, et al., 2007)

b) (Johansson, et al., 2003)

c) (Andersson, et al., 2006)

d) (Sternbeck, et al., 2007a)

\section{Conclusion:}

The compound was monitored in Norway in the Oslo region and in three studies throughout Sweden. The compound was detected in several matrices:

- STP sludge in high values in both countries. The concentration detected in one location in Sweden was very high

- STP effluent in one sample in Norway and in a few samples in Sweden

${ }^{3}$ Fish for human consumption

${ }^{4}$ Includes 4 samples from farms.

${ }^{5}$ Includes 7 samples at proximity of farms.

${ }^{6}$ Includes 1 treated sample 
- In one landfill leachate sample in Sweden

- Hospital waste water in Sweden

- STP influent in Sweden

\subsubsection{Erythromycin}

CAS nr.: 114-07-8

Info: macrolide antibiotic

Toxicity data: NA

PNEC $=0,02 \mu \mathrm{g} / \mathrm{l}$ (Pedersen, et al., 2007)
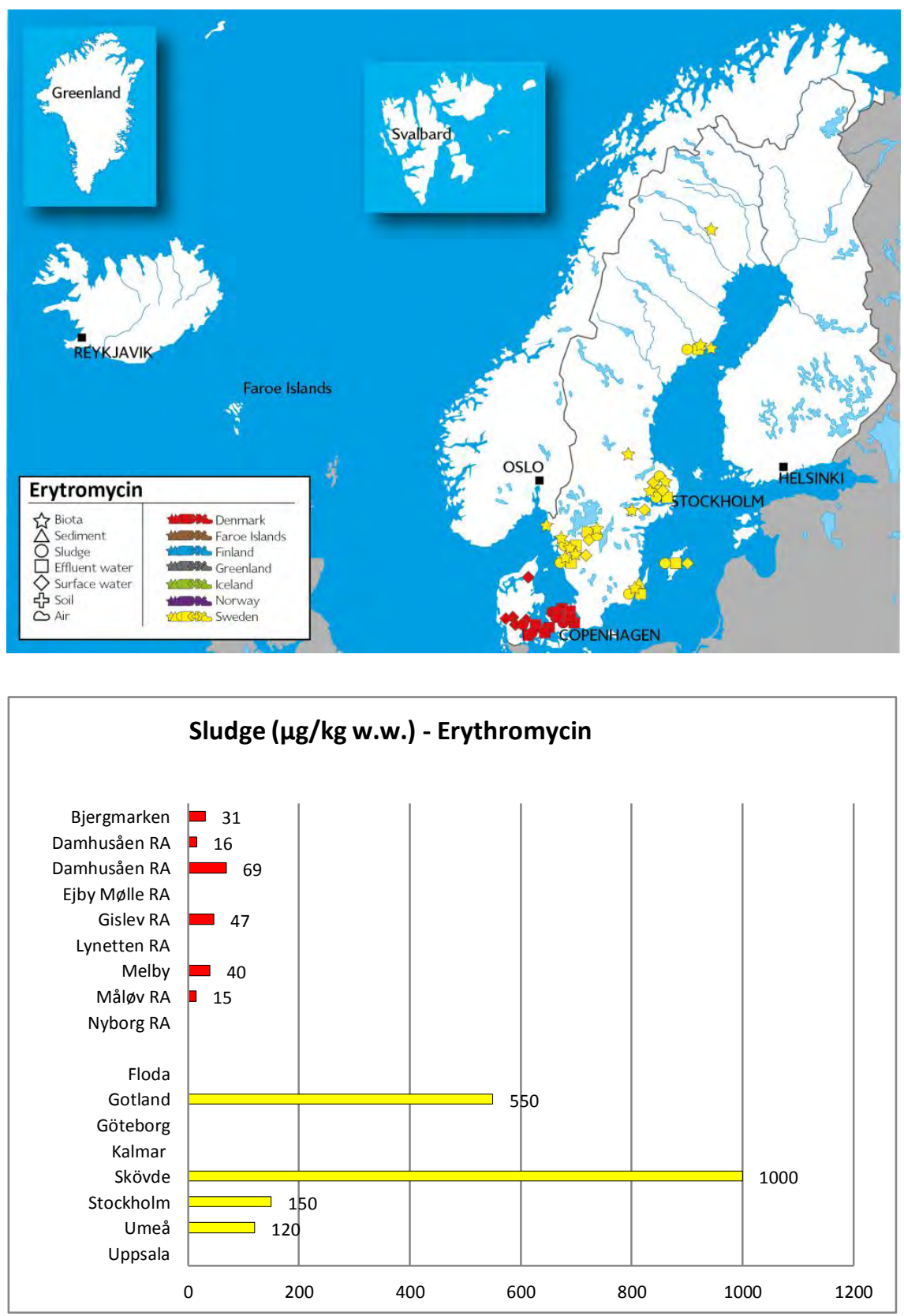


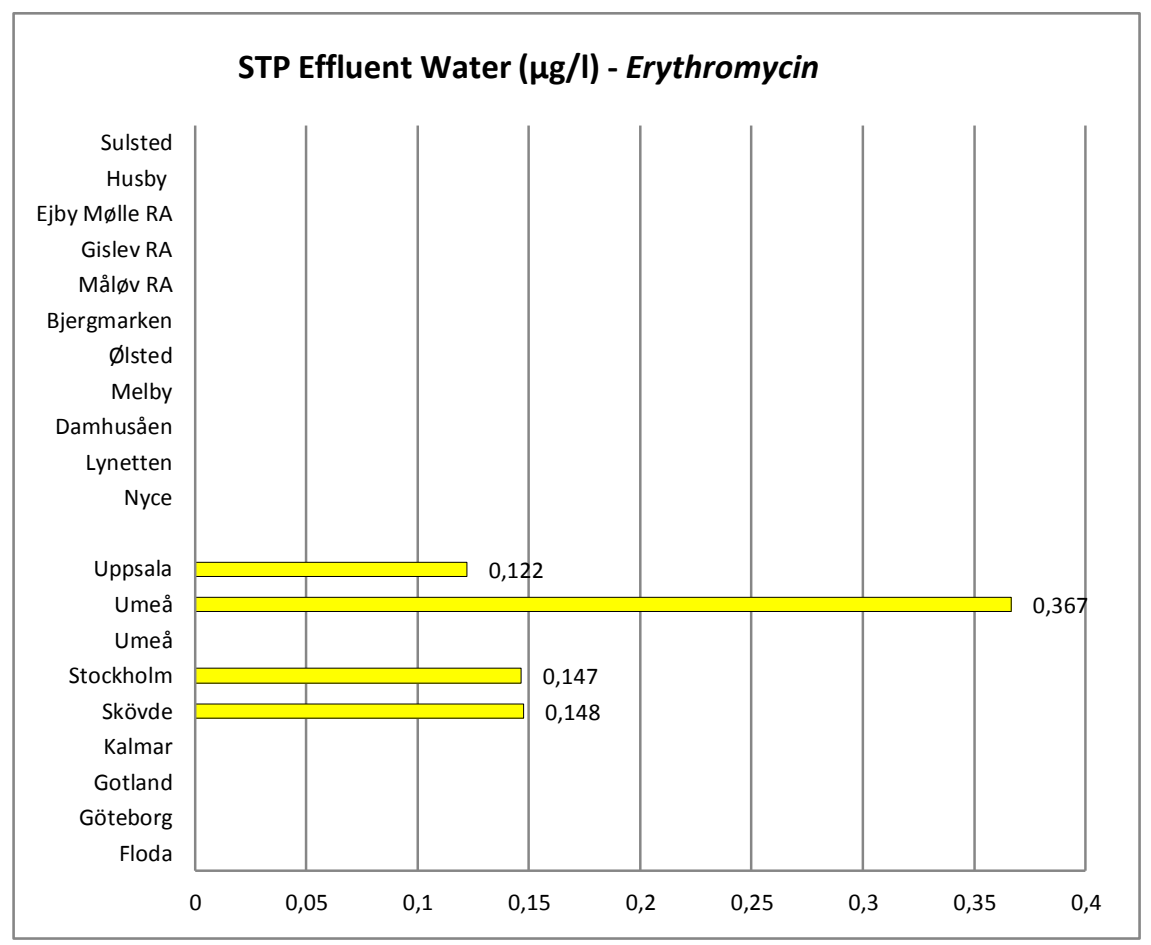

\begin{tabular}{|c|c|c|c|c|}
\hline \multirow{2}{*}{$\begin{array}{l}\text { Erythromycin } \\
\text { Matrix }\end{array}$} & \multicolumn{2}{|c|}{ Denmark } & \multicolumn{2}{|c|}{ Sweden } \\
\hline & $\min$ & Nr.S & $\min$ & Nr.S \\
\hline & $\max$ & (ref) & $\max$ & (ref) \\
\hline & $A D$ & & $A D$ & \\
\hline Biota & & & nd & $24(b c)$ \\
\hline \multirow[t]{3}{*}{ STP sludge $(\mu \mathrm{g} / \mathrm{kg} \mathrm{dw})$} & nd & $10(a)$ & nd & 10 (bc) \\
\hline & 69 & & 1000 & \\
\hline & 36,3 & & 455 & \\
\hline \multirow[t]{3}{*}{ STP effluent $(\mu \mathrm{g} / \mathrm{l})$} & nd & $9(a)$ & nd & $18(\mathrm{bc})$ \\
\hline & & & 0,53 & \\
\hline & & & 0,19 & \\
\hline \multirow[t]{3}{*}{ Surface water $(\mu \mathrm{g} / \mathrm{l})$} & nd & $16^{7}(\mathrm{a})$ & nd & 15 (b) \\
\hline & & & 0,065 & \\
\hline & & & 0,059 & \\
\hline Sediment & & & nd & 1 (b) \\
\hline Drinking water & & & nd & 6 (c) \\
\hline \multirow[t]{3}{*}{ STP influent ( $\mu \mathrm{g} / \mathrm{l})$} & nd & 9 (a) & nd & $17(\mathrm{bc})$ \\
\hline & & & 2,1 & \\
\hline & & & 0,46 & \\
\hline
\end{tabular}

${ }^{7}$ Upstream and downstream of fish farms 


\begin{tabular}{lcccc}
\hline Erythromycin & Denmark & & Sweden \\
\hline Hospital WW & & & nd & 2 (b) \\
Warning system for pesticide leaching to groundwater & nd & $8(a)$ & \\
Drain water & nd & 1 (a) \\
Groundwater & nd & $3^{8}$ (a) \\
\hline
\end{tabular}

\section{References:}

a) (Mogensen, et al., 2008)

b) (Johansson, et al., 2003)

c) (Fick, et al., 2011)

\section{Conclusion:}

The compound was monitored in one study throughout Denmark and two studies throughout Sweden. It was detected in several matrices:

- STP sludge in both countries. The values detected in Sweden were higher (at least twice as high)

- STP effluent water in Sweden but not in Denmark. The compound was detected in approx. $50 \%$ of all locations monitored and in concentrations above the PNEC

- Surface water in Sweden but not in Denmark. The average detected value was above the PNEC

- STP influent water in Sweden but not in Denmark 


\subsubsection{Norfloxacin}

CAS nr.: 70458-96-7

Info: synthetic chemotherapeutic antibacterial agent

Toxicity data: NA

$P N E C=\mathrm{NA}$
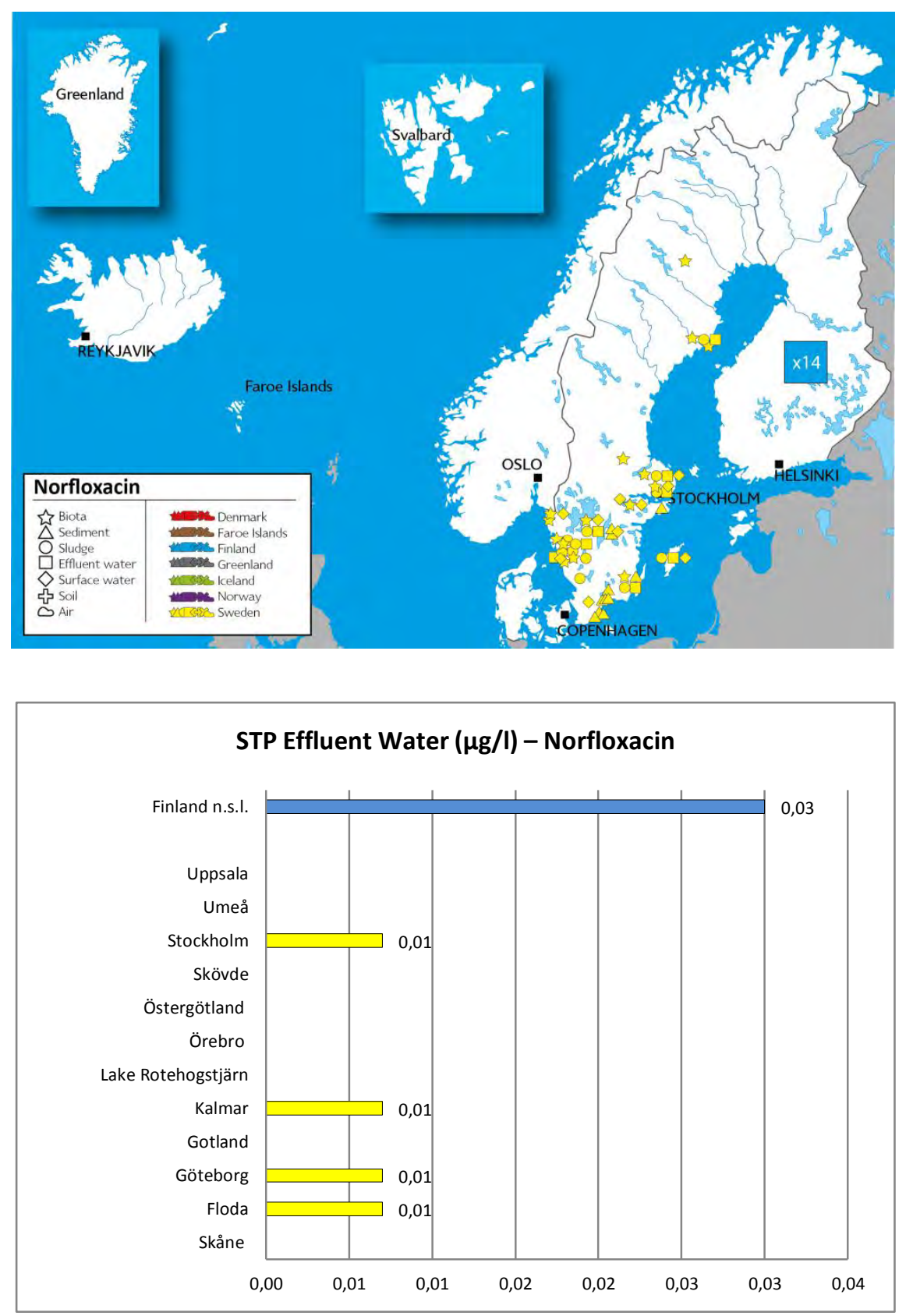


\begin{tabular}{|c|c|c|c|c|}
\hline \multirow{2}{*}{$\begin{array}{l}\text { Norfloxacin } \\
\text { Matrix }\end{array}$} & \multicolumn{2}{|c|}{ Finland } & \multicolumn{2}{|c|}{ Sweden } \\
\hline & $\min$ & Nr.S & $\min$ & Nr.S \\
\hline & $\max$ & (ref) & $\max$ & (ref) \\
\hline & $A D$ & & $A D$ & \\
\hline Biota & & & nd & $24(\mathrm{bc})$ \\
\hline \multirow[t]{3}{*}{ STP sludge ( $\mathrm{mg} / \mathrm{kg} \mathrm{dw}$ ) } & & & nd & \multirow{3}{*}{41 (bcde) } \\
\hline & & & 6,1 & \\
\hline & & & 1,93 & \\
\hline \multirow[t]{3}{*}{ STP effluent $(\mu \mathrm{g} / \mathrm{l})$} & NA & $14(\mathrm{a})$ & nd & \multirow{3}{*}{$18(b c$} \\
\hline & 0,03 & & 0,007 & \\
\hline & NA & & 0,007 & \\
\hline Surface water & & & nd & 45 (bf) \\
\hline Sediment & & & nd & 20 (bf) \\
\hline \multirow[t]{3}{*}{ Drinking water $(\mu \mathrm{g} / \mathrm{l})$} & & & nd & \multirow{3}{*}{$6(b)$} \\
\hline & & & 0,01 & \\
\hline & & & 0,01 & \\
\hline \multirow[t]{3}{*}{ STP Influent $(\mu \mathrm{g} / \mathrm{l})$} & NA & $14(\mathrm{a})$ & nd & \multirow{3}{*}{$17(b c)$} \\
\hline & NA & & 0,17 & \\
\hline & 0,12 & & 0,08 & \\
\hline Surface water & & & nd & 43 (bf) \\
\hline \multirow[t]{3}{*}{ Hospital WW $(\mu \mathrm{g} / \mathrm{l})$} & & & nd & \multirow{3}{*}{$2(c)$} \\
\hline & & & 0,8 & \\
\hline & & & 0,8 & \\
\hline Groundwater by farms & & & nd & 2 (f) \\
\hline Manure & & & nd & $6(f)$ \\
\hline Soil from farms & & & nd & $6(\mathrm{f})$ \\
\hline
\end{tabular}

\section{References:}

a) (Vieno, 2008)

b) (Fick, et al., 2011)

c) (Johansson, et al., 2003)

d) (Haglund \& Olofsson, 2006)

e) (Haglund \& Olofsson, 2007)

f) (Sternbeck, et al., 2007a)

\section{Conclusion:}

The compound was monitored in 14 STPs in Finland and five studies, mainly in the south of Sweden. It was detected in several matrices:

- STP sludge in Sweden

- STP effluent water in Finland and in one sample in Sweden

- In one drinking water sample in Sweden

- STP influent in both countries

- In one hospital waste water sample in Sweden 


\subsubsection{Ofloxacin}

CAS nr.: 82419-36-1

Info: Synthetic chemotherapeutic antibiotic of the fluoroquinolone drug class

Toxicity data: NA

$P N E C=\mathrm{NA}$

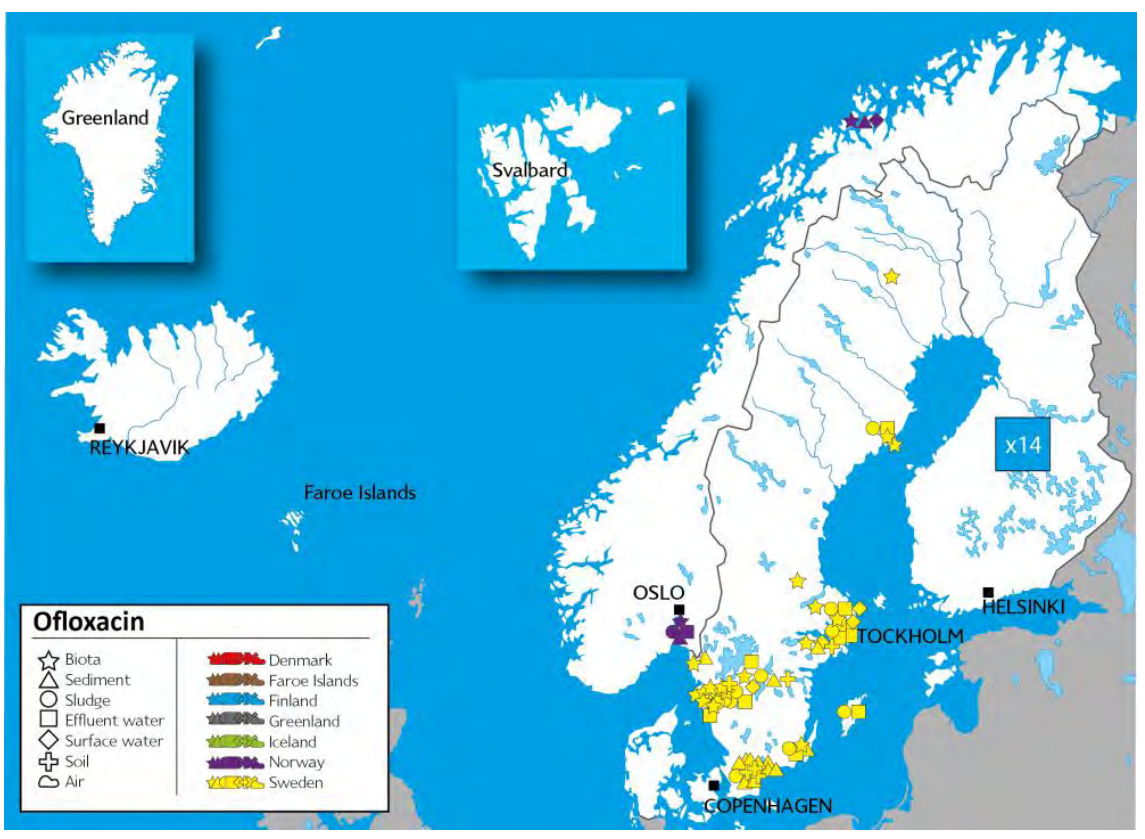

Sludge ( $\mu \mathrm{g} / \mathrm{kg}$ w.w.) - Ofloxacin

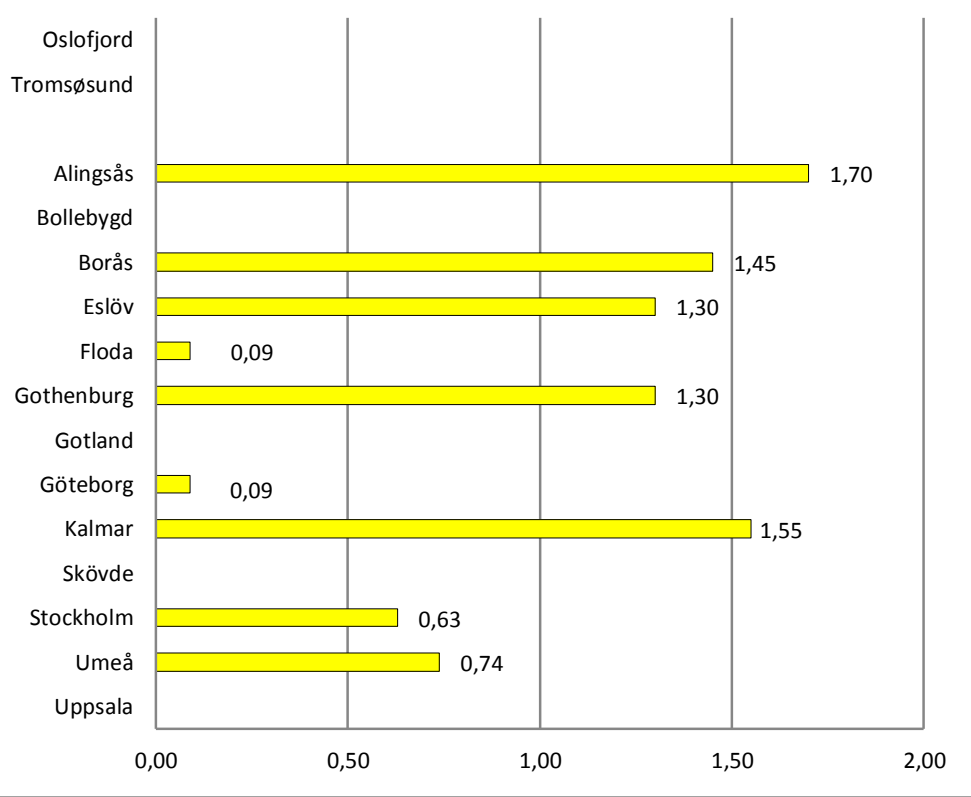




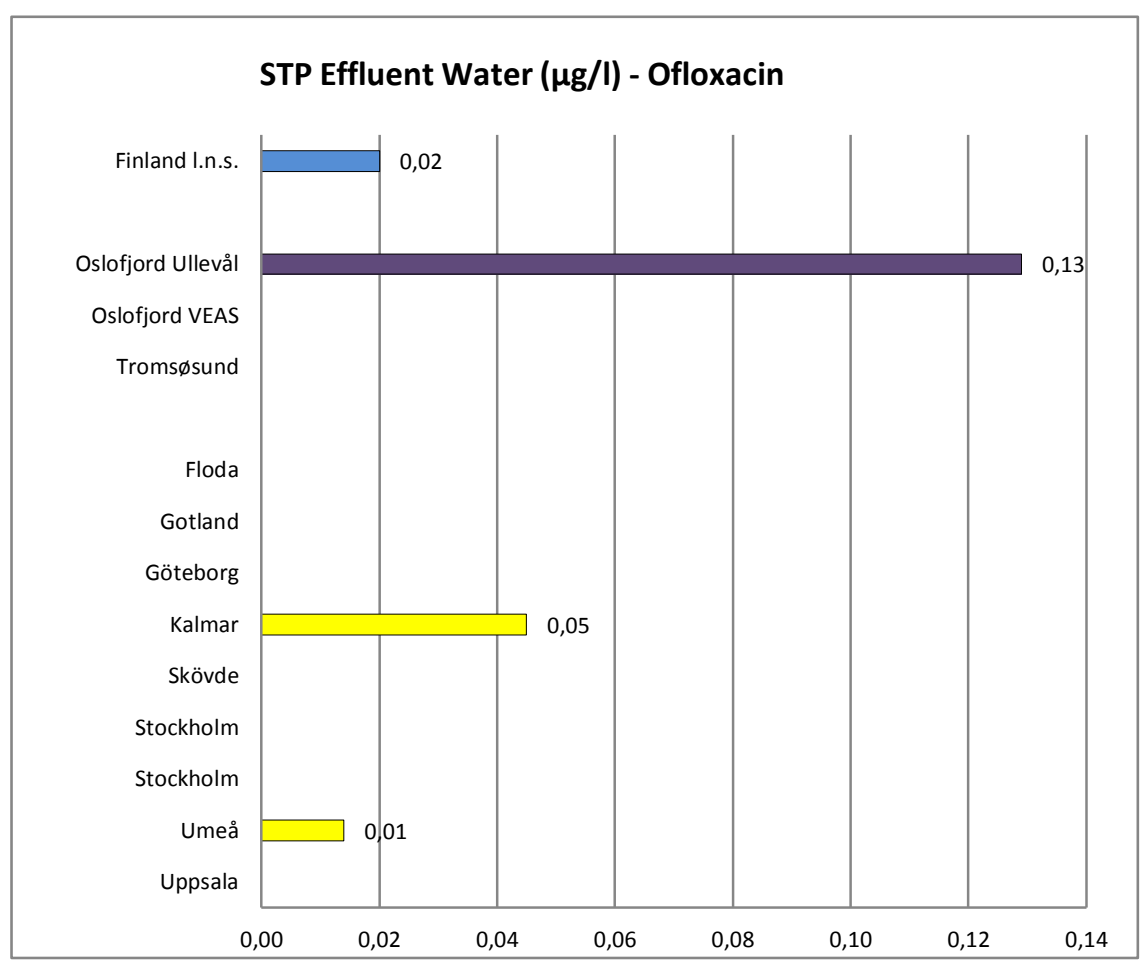

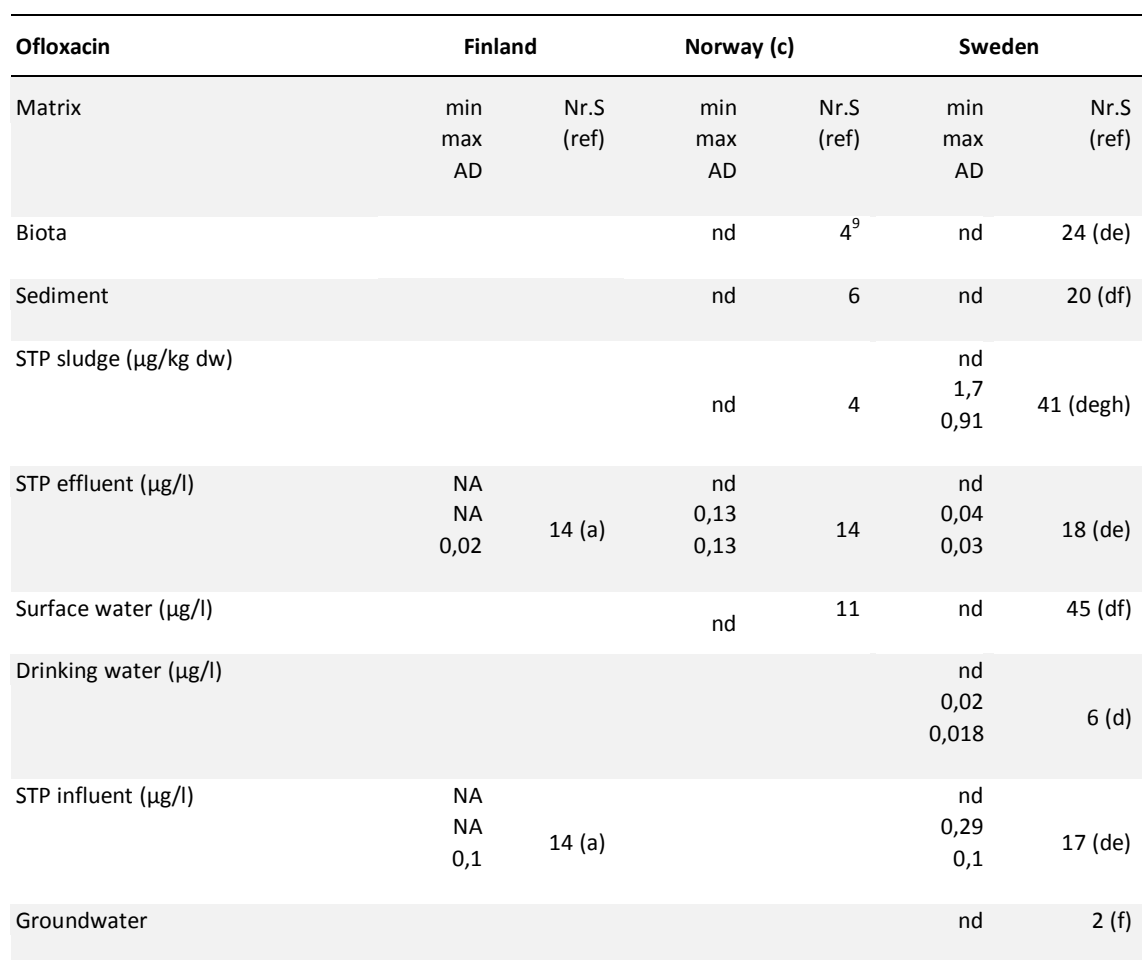

${ }^{9}$ Mussel 


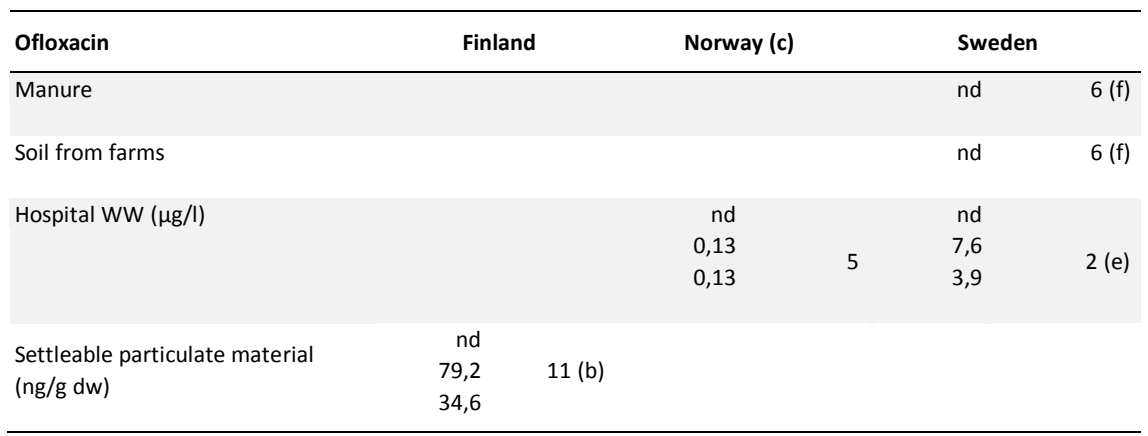

\section{References:}
a) (Vieno, 2008)
b) (Lahti \& Oikari, 2011)
c) (Schlabach, et al., 2009)
d) (Fick, et al., 2011)
e) (Johansson, et al., 2003)
f) (Sternbeck, et al., 2007a)
g) (Haglund \& Olofsson, 2006)
h) (Haglund \& Olofsson, 2007)

\section{Conclusion:}

The compound has been monitored in one study in Norway, two in Finland and five in Sweden. It was detected in several matrices:

- STP sludge in Sweden but not in Norway

- STP effluent in Finland, Sweden and Norway

- Drinking water in Sweden

- Settleable particulate material in Finland

- Hospital waste water in Sweden and in one sample in Norway

- STP influent in Finland and Sweden 


\subsubsection{Oxytetracycline}

CAS nr. 2058-46-0

Info: Broadspectrum tetracycline antibiotic

Toxicity data: NA

$P N E C=20 \mu \mathrm{g} / \mathrm{l}$

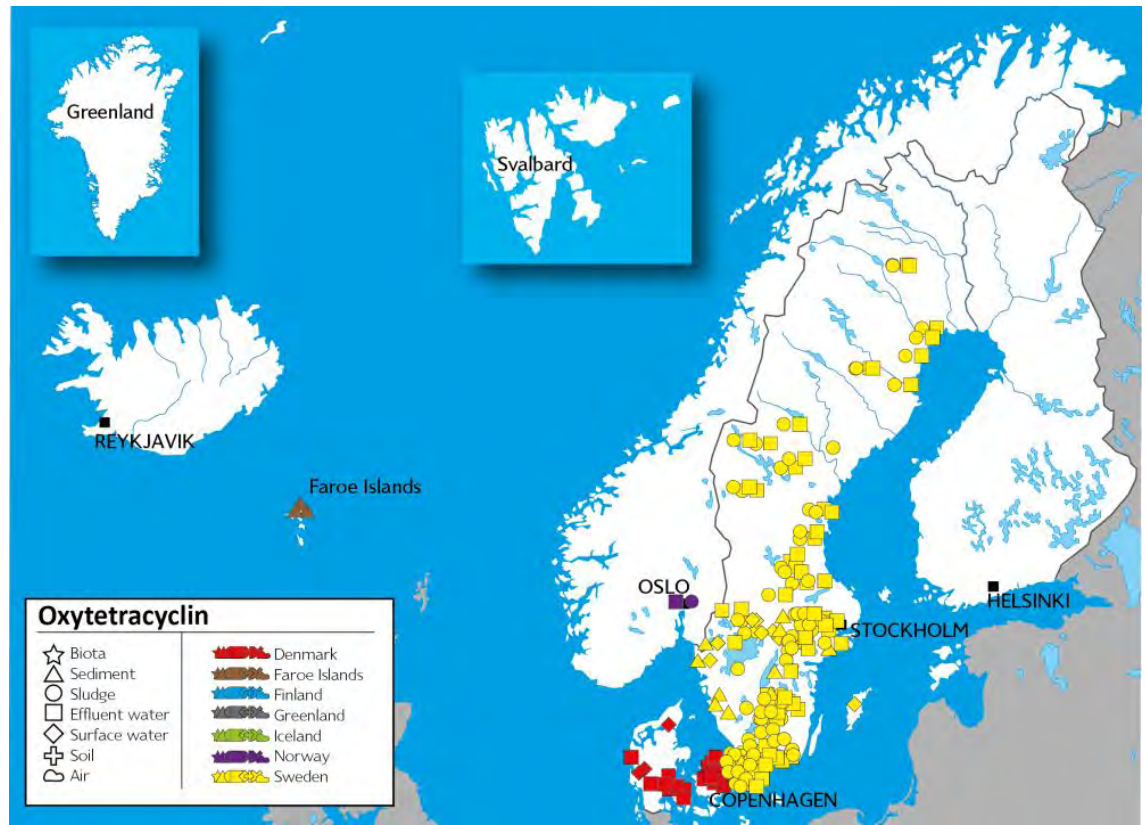




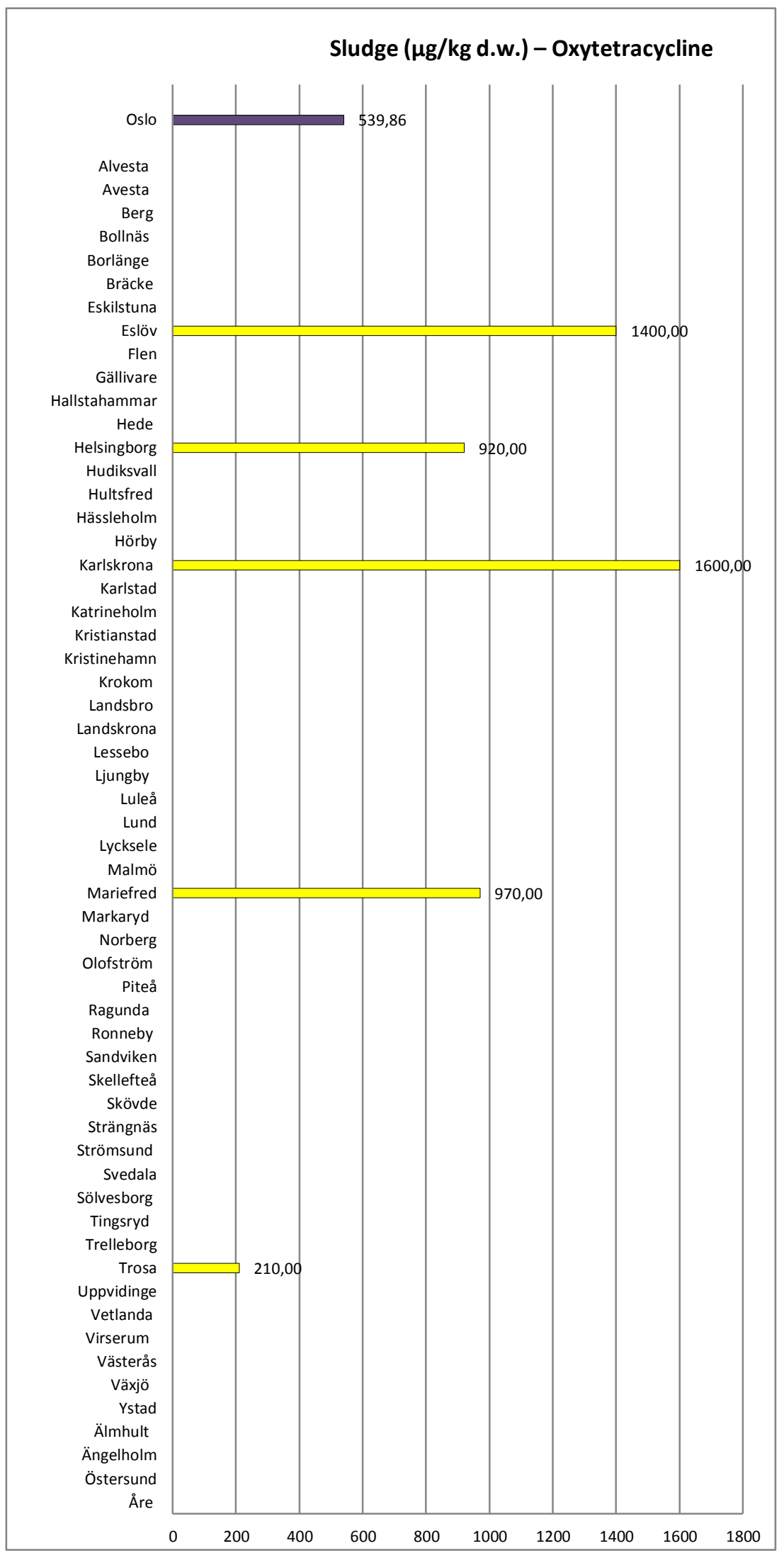




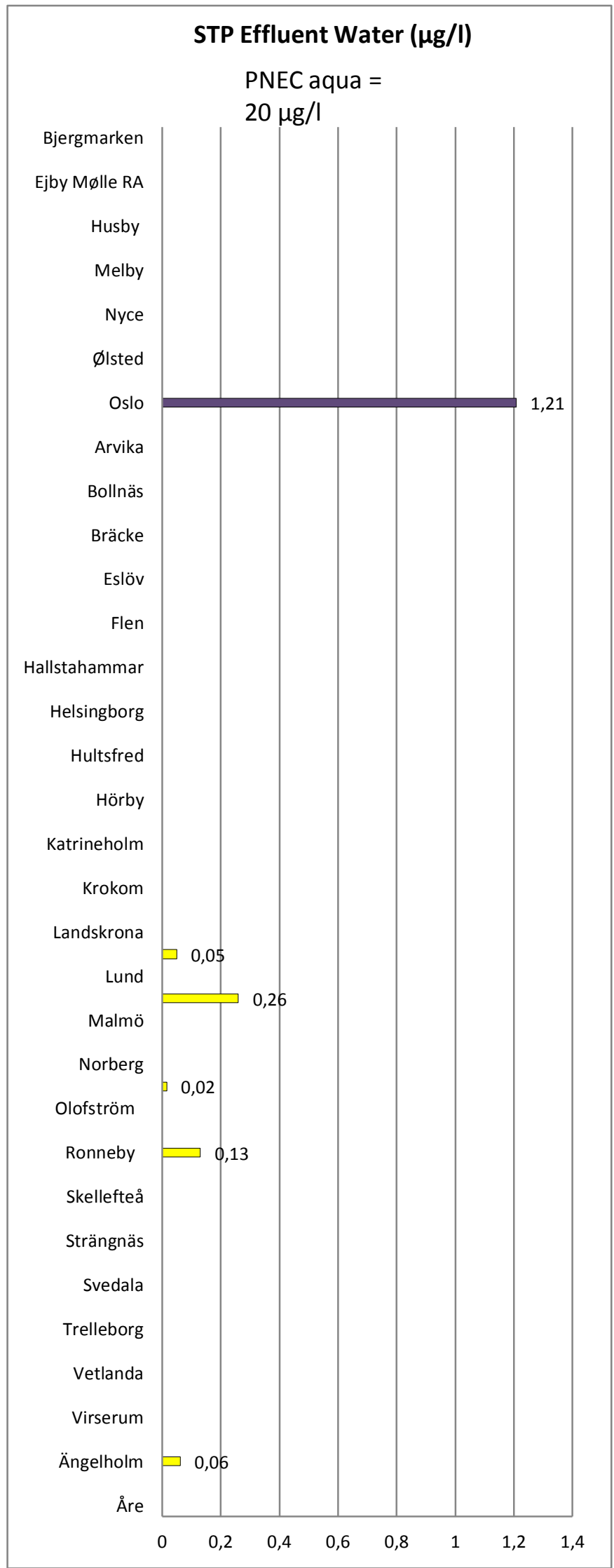




\begin{tabular}{|c|c|c|c|c|c|c|c|c|c|c|}
\hline $\begin{array}{l}\text { Oxytetracycline } \\
\text { Matrix }\end{array}$ & \multicolumn{2}{|c|}{ Denmark (a) } & \multicolumn{2}{|c|}{ Norway (b) } & \multicolumn{2}{|c|}{ Sweden } & \multicolumn{2}{|c|}{ Faroe Islands (e) } & \multicolumn{2}{|c|}{ Finland (f) } \\
\hline & $\begin{array}{r}\min \\
\max \\
A D\end{array}$ & $\begin{array}{l}\text { Nr.S } \\
\text { (ref) }\end{array}$ & $\begin{array}{r}\min \\
\max \\
A D\end{array}$ & $\begin{array}{l}\text { Nr.S } \\
\text { (ref) }\end{array}$ & $\begin{array}{r}\min \\
\max \\
A D\end{array}$ & $\begin{array}{l}\text { Nr.S } \\
\text { (ref) }\end{array}$ & $\begin{array}{r}\min \\
\max \\
A D\end{array}$ & $\begin{array}{l}\text { Nr.S } \\
\text { (ref) }\end{array}$ & $\begin{array}{r}\min \\
\max \\
A D\end{array}$ & $\begin{array}{l}\text { Nr.S } \\
\text { (ref) }\end{array}$ \\
\hline Biota & & & & & nd & $3^{10}(\mathrm{c})$ & & & & \\
\hline Surface water & nd & $16^{11}$ & & & nd & $47^{12}(\mathrm{~cd})$ & & & & \\
\hline Sediment (ng/g dw) & & & & & nd & $26(\mathrm{~cd})$ & $\begin{array}{l}0,07 \\
0,35 \\
0,18\end{array}$ & 12 & & \\
\hline STP sludge (mg/kg dw) & & & $\begin{array}{r}\text { nd } \\
2,07 \\
0,63\end{array}$ & 7 & $\begin{array}{r}\text { nd } \\
1,6 \\
1,02\end{array}$ & 61 (c) & & & & \\
\hline STP effluent $(\mu \mathrm{g} / \mathrm{l})$ & nd & 9 & $\begin{array}{r}\text { nd } \\
1,21 \\
1,21\end{array}$ & 7 & $\begin{array}{r}\text { nd } \\
0,26 \\
0,10\end{array}$ & 52 (c) & & & & \\
\hline STP Influent $(\mu \mathrm{g} / \mathrm{l})$ & nd & 9 & & & $\begin{array}{r}\text { nd } \\
0,79 \\
0,32\end{array}$ & 19 (c) & & & & \\
\hline $\begin{array}{l}\text { Warning system for } \\
\text { pesticide leaching to } \\
\text { groundwater ( } \mathrm{mg} / \mathrm{kg} \mathrm{dw} \text { ) }\end{array}$ & $\begin{array}{r}\text { nd } \\
0,51 \\
0,51\end{array}$ & 9 & & & & & & & & \\
\hline $\begin{array}{l}\text { Settleable particulate } \\
\text { material (ng/g dw) }\end{array}$ & & & & & & & & & $\begin{array}{r}\text { nd } \\
31,4 \\
31,4\end{array}$ & 11 \\
\hline Pig slurry & nd & 4 & & & & & & & & \\
\hline Drain water & nd & 1 & & & & & & & & \\
\hline Groundwater & nd & $3^{13}$ & & & nd & $2^{14}(c)$ & & & & \\
\hline Farm soil & & & & & nd & 6 (c) & & & & \\
\hline Manure & & & & & nd & $11(\mathrm{~cd})$ & & & & \\
\hline Landfill leachates & & & & & nd & 8 (c) & & & & \\
\hline Hospital WW & & & & & nd & 7 (c) & & & & \\
\hline
\end{tabular}

\section{References:}

a) (Mogensen, et al., 2008)

b) (Thomas, et al., 2007)

c) (Andersson, et al., 2006)

d) (Sternbeck, et al., 2007a)

e) (Gustavson, et al., 2009)

f) (Lahti \& Oikari, 2011)

10 Fish for human consumption

$11 \mathrm{Up}$ and downstream of fish farms

12 Includes 9 samples at proximity of farms. The compound was detected in only one sample at proximity of a

cattle farm.

${ }^{13}$ Include 2 samples at proximity of hospitals

${ }^{14}$ At proximity of farms 


\section{Conclusion:}

The compound was monitored in two Swedish studies, and one Norwegian, one Danish, one Faroe Islands, and one Finnish study. The compound was detected in several matrices:

- STP sludge in Norway and Sweden

- STP effluent in Norway and Sweden but not Denmark. The values detected in Norway were higher than in Sweden

- STP influent in Sweden but not in Denmark

- In one sample for "pesticide leaching warning system"

- In settleable particulate material in Finland

\subsubsection{Sulfadiazine}

CAS nr.: 68-35-9

Info: a sulfonamide antibiotic

Toxicity data:

EC50 (M. aeruginosa) $=0,135 \mathrm{mg} / \mathrm{l}$

EC50 (S. capricornutum) $=7,8 \mathrm{mg} / \mathrm{l}$

EC50 (R. Salina) $=403 \mathrm{mg} / \mathrm{l}$

EC50 $72 \mathrm{hr}$ (crustaceans) = $57 \mathrm{mg} / \mathrm{l}$

(Hellström \& Kreuger, 2005)

$P N E C=\mathrm{NA}$

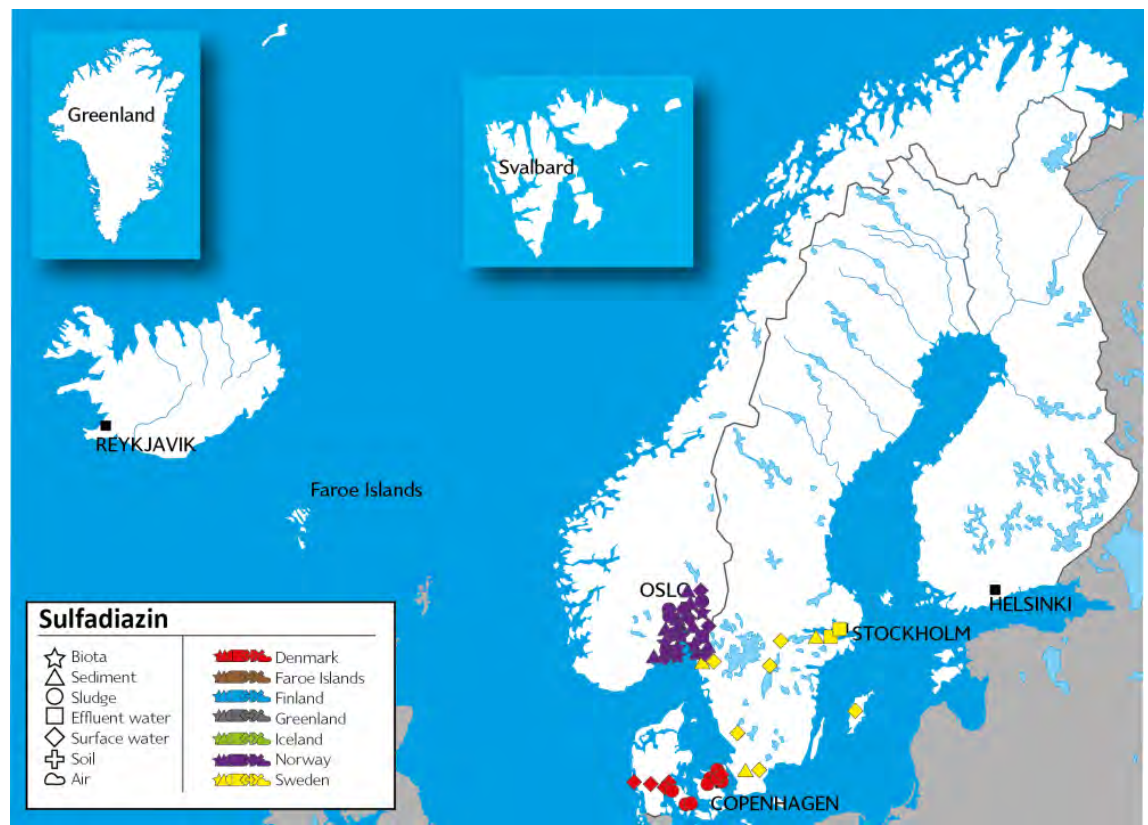




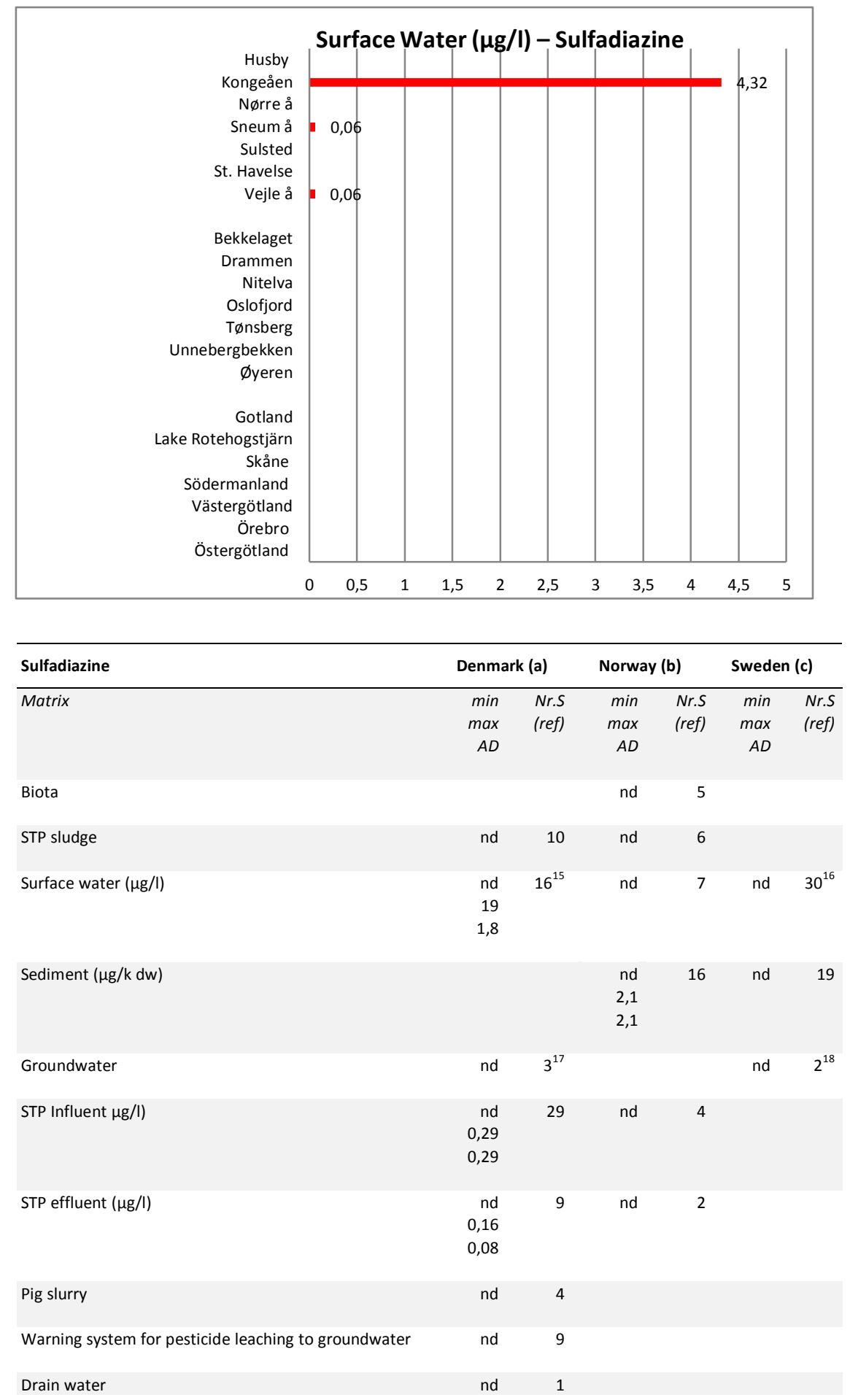

$15 \mathrm{Up}$ and downstream of fish farms

16 Includes 2 samples at proximity of farms

17 includes 2 samples at proximity of hospitals

${ }^{18}$ At proximity of farms 


\begin{tabular}{lcrr}
\hline Sulfadiazine & Denmark (a) & Norway (b) & \multicolumn{2}{c}{ Sweden (c) } \\
\hline Landfill leachates, water & nd & $9^{19}$ & \\
Landfill leachates, sediment & nd & 4 & \\
Manure & & nd & 6 \\
Farm soil & & nd & 6 \\
\hline
\end{tabular}

\section{References:}

a) (Mogensen, et al., 2008)

b) (Møskeland, et al., 2006)

c) (Sternbeck, et al., 2007a)

\section{Conclusion:}

The compound was monitored in one Danish, one Norwegian and one Swedish study and was detected in several matrices:

- Surface water in Denmark but not in Norway or Sweden

- Sediment in Norway but not in Sweden

- STP effluent and influent in Denmark, not Norway

\subsubsection{Sulfamethizol}

CAS nr.: 144-82-1

Info: Sulfonamide antibiotic Toxicity data: NA

$P N E C=\mathrm{NA}$

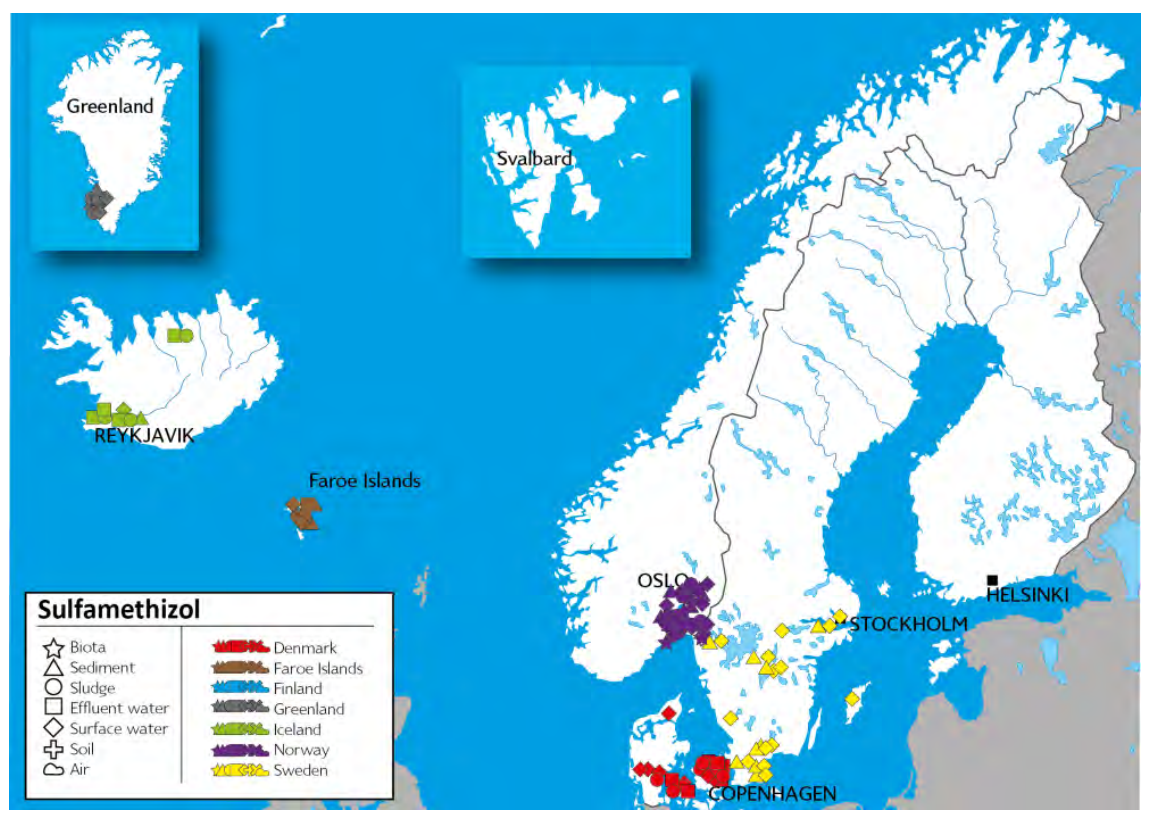

19 includes 5 treated samples 


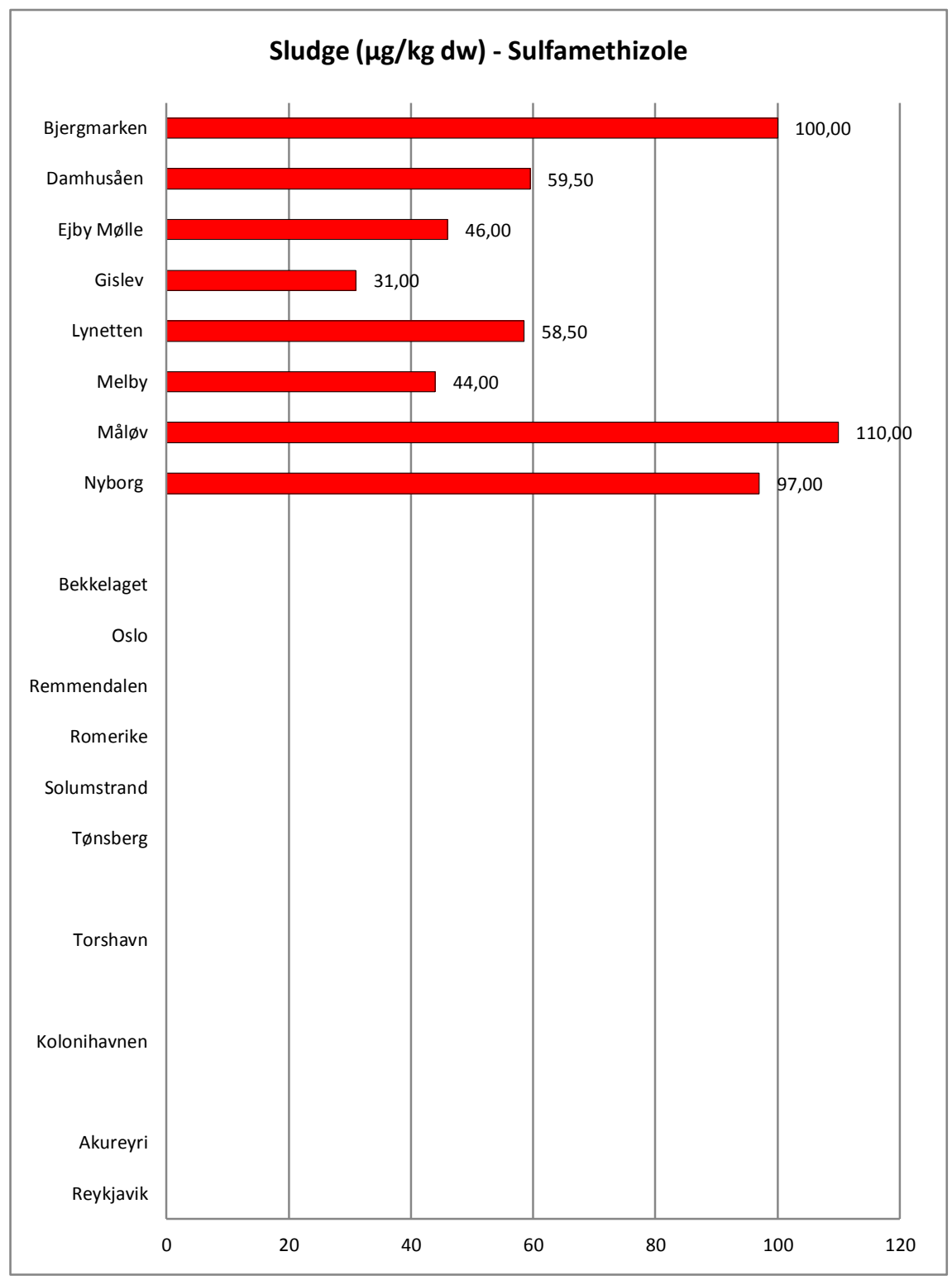




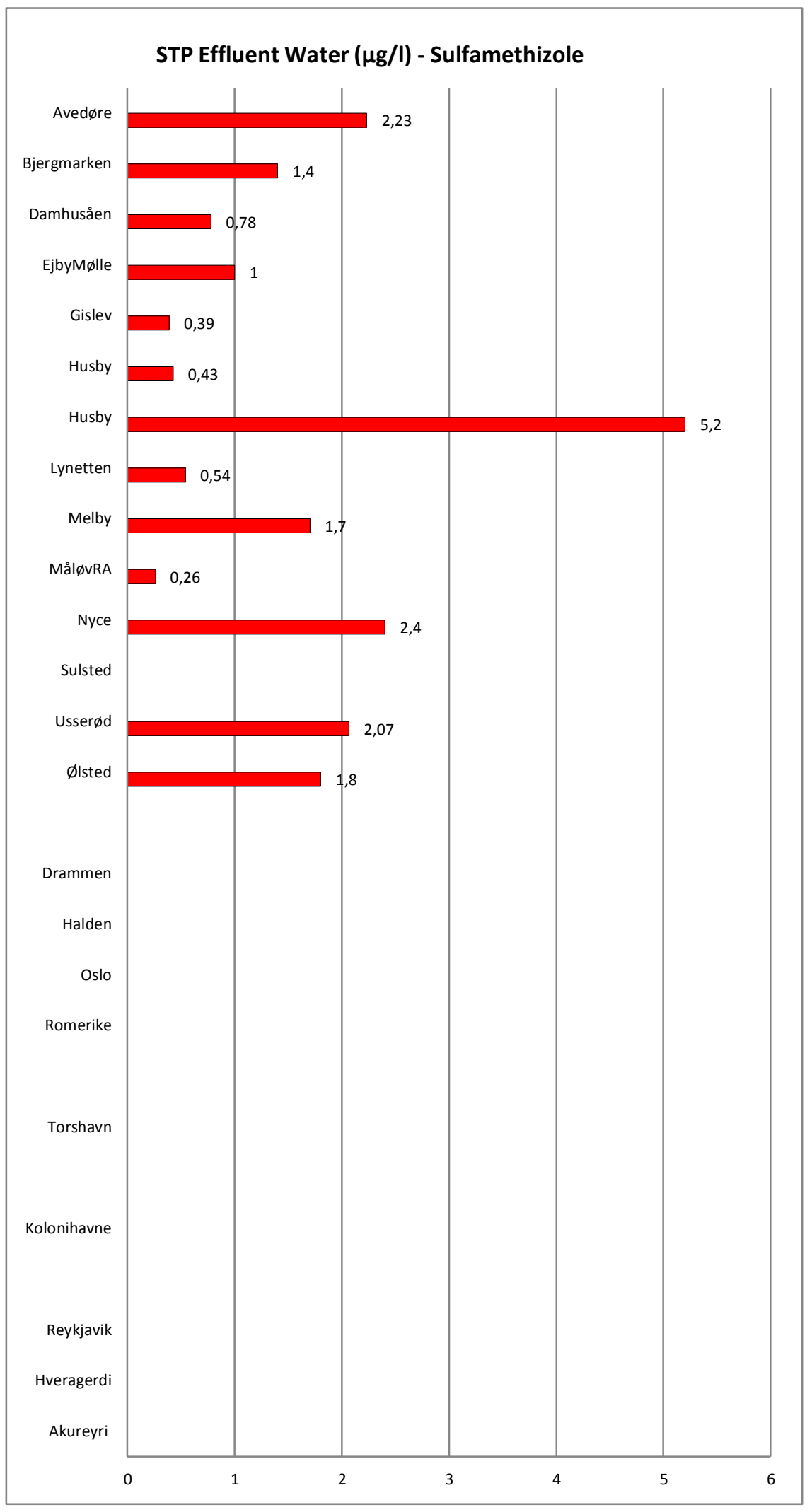




\begin{tabular}{|c|c|c|c|c|c|c|c|c|c|c|c|c|}
\hline \multirow{2}{*}{$\begin{array}{l}\text { Sulfamethizol } \\
\text { Matrix }\end{array}$} & \multicolumn{2}{|c|}{ Norway } & \multicolumn{2}{|c|}{ Denmark } & \multicolumn{2}{|c|}{ Sweden } & \multicolumn{2}{|c|}{ Faroe Islands } & \multicolumn{2}{|c|}{ Greenland } & \multicolumn{2}{|c|}{ Iceland } \\
\hline & $\begin{array}{r}\min \\
\max \\
A D\end{array}$ & $\begin{array}{l}\text { Nr.S } \\
\text { (ref) }\end{array}$ & $\begin{array}{r}\min \\
\max \\
A D\end{array}$ & $\begin{array}{l}\text { Nr.S } \\
\text { (ref) }\end{array}$ & $\begin{array}{r}\min \\
\max \\
A D\end{array}$ & $\begin{array}{l}\text { Nr.S } \\
\text { (ref) }\end{array}$ & $\begin{array}{r}\min \\
\max \\
A D\end{array}$ & $\begin{array}{l}\text { Nr.S } \\
\text { (ref) }\end{array}$ & $\begin{array}{r}\min \\
\max \\
A D\end{array}$ & $\begin{array}{l}\text { Nr.S } \\
\text { (ref) }\end{array}$ & $\begin{array}{r}\min \\
\max \\
A D\end{array}$ & $\begin{array}{l}\text { Nr.S } \\
\text { (ref) }\end{array}$ \\
\hline Biota & nd & 6 (a) & & & & & & & & & & \\
\hline STP sludge $(\mu \mathrm{g} / \mathrm{l})$ & nd & 6 (a) & $\begin{array}{r}31 \\
110 \\
66,4\end{array}$ & 10 (c) & & & nd & $2(\mathrm{e})$ & nd & $2(\mathrm{e})$ & nd & $3(\mathrm{e})$ \\
\hline Sediment & nd & 12 (a) & & & & & nd & $3(\mathrm{e})$ & nd & $2(\mathrm{e})$ & & \\
\hline Landfill leachates, sediment & nd & 4 (a) & & & & & & & & & & \\
\hline Landfill leachates, water & nd & $9^{20}(a)$ & & & & & & & & & & \\
\hline Surface water & nd & $8(a)$ & nd & $16^{21}(\mathrm{c})$ & nd & $30^{22}$ & nd & $5(\mathrm{e})$ & nd & $5(\mathrm{e})$ & & \\
\hline Groundwater & & & nd & $3^{23}(\mathrm{c})$ & nd & $2^{24}$ & & & & & & \\
\hline STP Influent & nd & $8(a)$ & & & & & nd & 1 (e) & nd & 1 (e) & nd & 5 (e) \\
\hline STP effluent $(\mu \mathrm{g} / \mathrm{l})$ & nd & 4 (a) & $\begin{array}{l}\text { nd } \\
5,2 \\
1,5\end{array}$ & $16(\mathrm{~cd})$ & & & nd & 1 (e) & nd & 1 (e) & nd & 5 (e) \\
\hline Sediment & & & & & nd & 19 & & & & & & \\
\hline Manure & & & & & nd & 6 & & & & & & \\
\hline Farm soil & & & & & nd & 6 & & & & & & \\
\hline Pig slurry & & & nd & (d) & & & & & & & & \\
\hline $\begin{array}{l}\text { Warning system for pesticide } \\
\text { leaching to groundwater }\end{array}$ & & & nd & 9 (c) & & & & & & & & \\
\hline Drain water $(\mu \mathrm{g} / \mathrm{l})$ & & & $\begin{array}{l}0,21 \\
0,21 \\
0,21\end{array}$ & 1 (c) & & & & & & & & \\
\hline Influent $(\mu \mathrm{g} / \mathrm{l})$ & & & $\begin{array}{r}0,34 \\
6,4 \\
2,9\end{array}$ & 9 (c) & & & & & & & & \\
\hline Hospital WW & & & & & & & nd & $3(\mathrm{e})$ & & & & \\
\hline
\end{tabular}

\section{References:}

a) (Møskeland, et al., 2006)

b) (Sternbeck, et al., 2007a)

c) (Mogensen, et al., 2008)

d) (Kjølholt, et al., 2003)

e) (Huber, et al., NA)

\footnotetext{
20 includes 5 treated samples

${ }^{21} \mathrm{Up}$ and downstream of fish farms

22 Including 2 at proximity of farms

232 samples at proximity of hospitals

${ }^{24}$ From farms
} 


\section{Conclusion:}

The compound was monitored in one Norwegian, one Swedish, two Danish studies and one international study covering Faroe Islands, Greenland and Iceland. It was not detected in any other country than Denmark. In Denmark, the compound was detected in STP sludge, effluent and influent as well as in the one drain water sample.

\subsubsection{Sulfamethoxazole}

CAS nr.:723-46-6

Info: Sulfonamide bacteriostatic antibiotic

Toxicity data: NA

$P N E C=\mathrm{NA}$

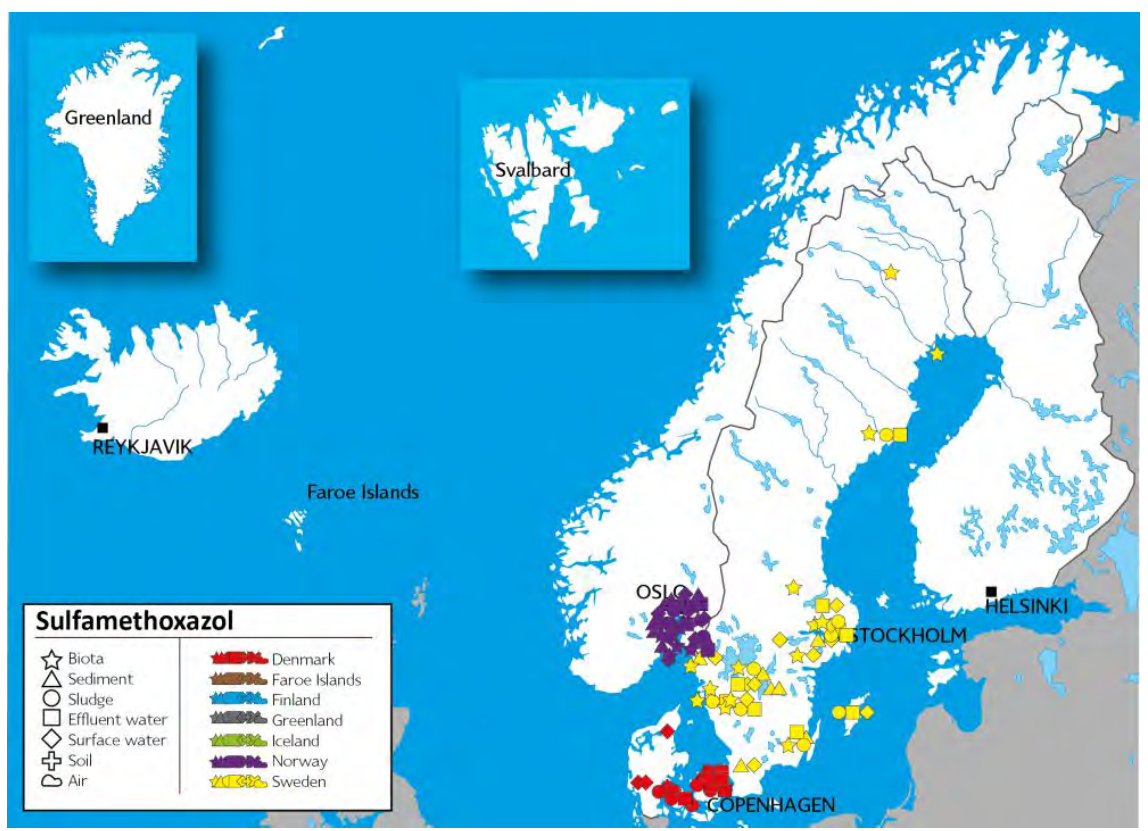




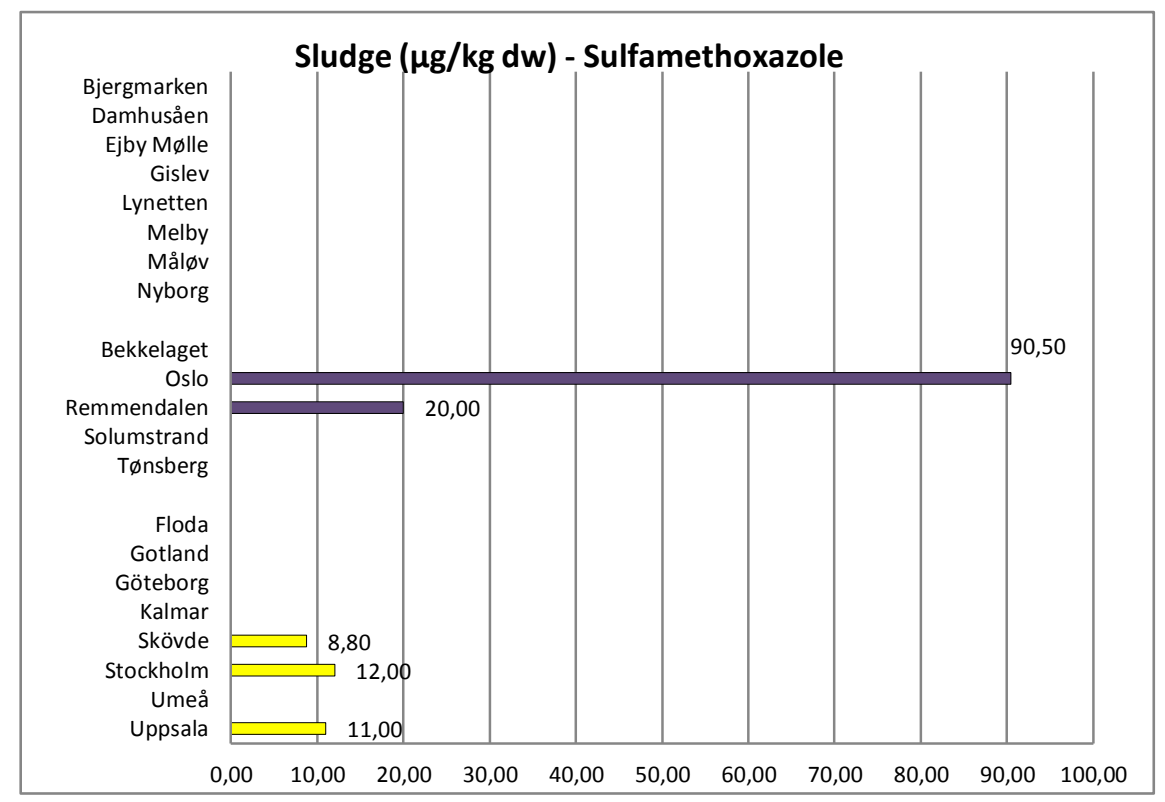




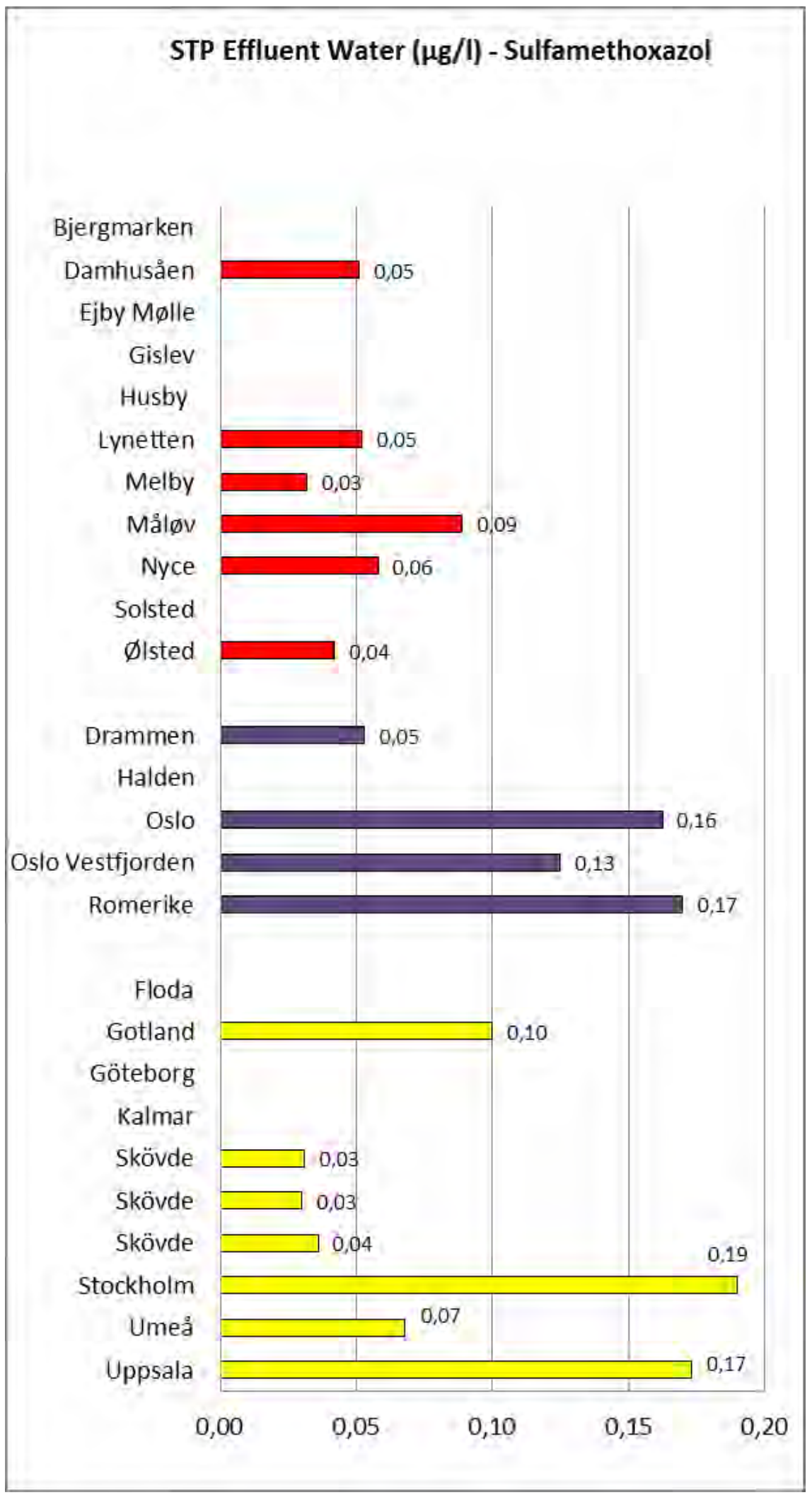




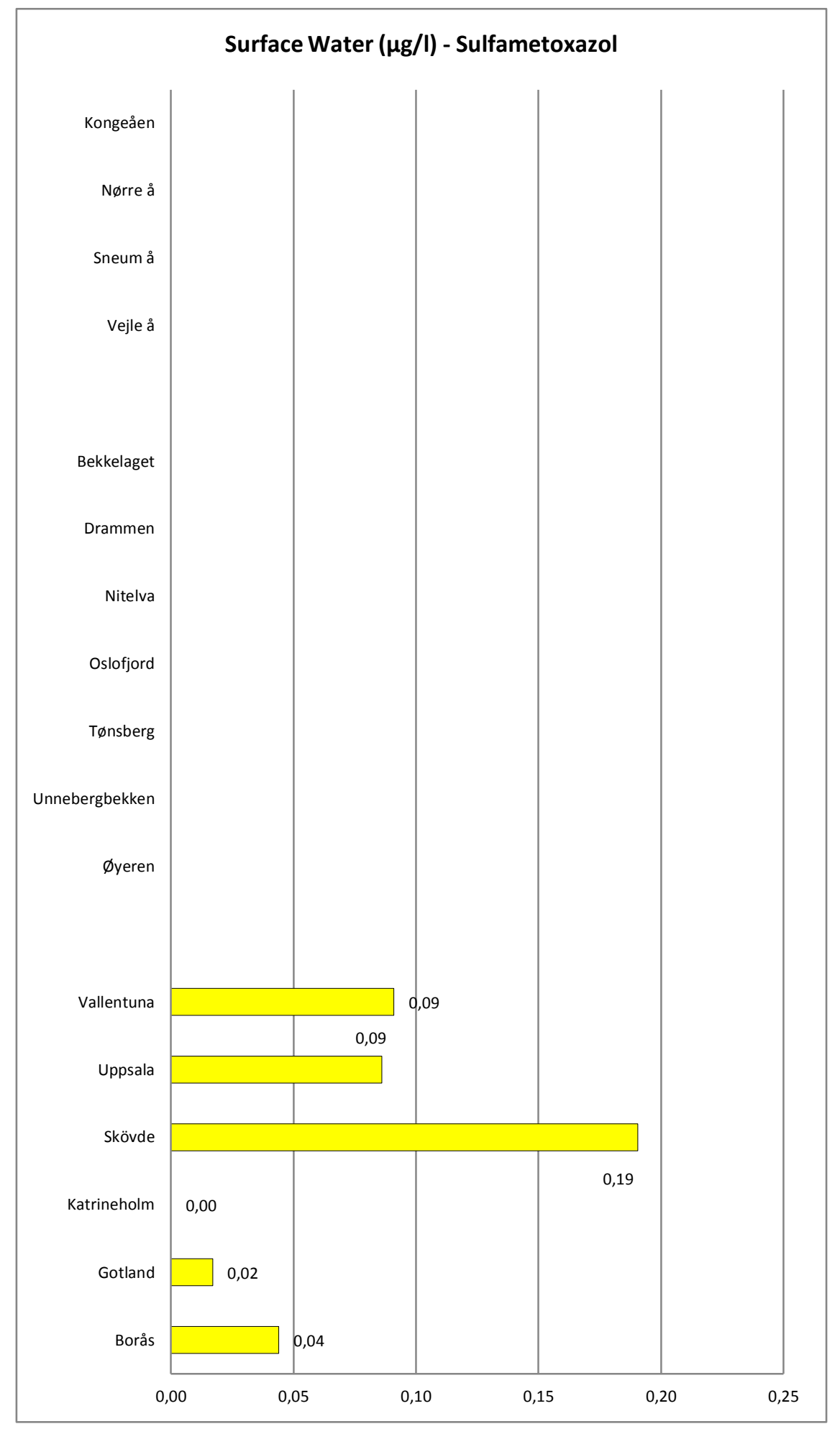




\begin{tabular}{|c|c|c|c|c|c|c|}
\hline \multirow{2}{*}{$\begin{array}{l}\text { Sulfamethoxazole } \\
\text { Matrix }\end{array}$} & \multicolumn{2}{|c|}{ Denmark (a) } & \multicolumn{2}{|c|}{ Norway } & \multicolumn{2}{|c|}{ Sweden } \\
\hline & $\min$ & Nr.S & $\min$ & $\mathrm{Nr} . \mathrm{S}$ & $\min$ & $\mathrm{Nr} . \mathrm{S}$ \\
\hline & $\max$ & (ref) & $\max$ & (ref) & $\max$ & (ref) \\
\hline & $A D$ & & $A D$ & & $A D$ & \\
\hline \multirow[t]{3}{*}{ Biota $(\mu \mathrm{g} / \mathrm{kg} \mathrm{dw})$} & & & \multirow{3}{*}{ nd } & \multirow{3}{*}{6 (b) } & nd & \multirow{3}{*}{24 (de) } \\
\hline & & & & & 13 & \\
\hline & & & & & 13 & \\
\hline Sediment & & & nd & $16(b)$ & nd & 20 (df) \\
\hline \multirow[t]{3}{*}{ STP sludge $(\mu \mathrm{g} / \mathrm{kg} \mathrm{dw})$} & \multirow{3}{*}{ nd } & \multirow{3}{*}{10} & nd & \multirow{3}{*}{$13(b c)$} & nd & \multirow{3}{*}{10 (de) } \\
\hline & & & 171 & & 12 & \\
\hline & & & 51,5 & & 10,6 & \\
\hline Drinking water & & & & & nd & 6 (d) \\
\hline \multirow[t]{3}{*}{ STP effluent $(\mu \mathrm{g} / \mathrm{l})$} & nd & \multirow{3}{*}{9} & nd & \multirow{3}{*}{11 (bc) } & nd & \multirow{3}{*}{18 (de) } \\
\hline & 0,09 & & 0,21 & & 0,29 & \\
\hline & 0,05 & & 0,13 & & 0,13 & \\
\hline \multirow[t]{3}{*}{ Surface water $(\mu \mathrm{g} / \mathrm{l})$} & \multirow{3}{*}{ nd } & \multirow{3}{*}{$16^{25}$} & \multirow{3}{*}{ nd } & \multirow{3}{*}{8 (b) } & nd & \multirow{3}{*}{45 (df) } \\
\hline & & & & & 0,62 & \\
\hline & & & & & 0,13 & \\
\hline Groundwater & nd & $3^{26}$ & & & nd & $2^{27}(f)$ \\
\hline Manure & & & & & nd & $6(f)$ \\
\hline Farm soil & & & & & nd & $6(f)$ \\
\hline \multirow[t]{3}{*}{ Hospital WW $(\mu \mathrm{g} / \mathrm{l})$} & & & & & nd & \multirow{3}{*}{2 (e) } \\
\hline & & & & & 6,6 & \\
\hline & & & & & 6,6 & \\
\hline \multirow[t]{3}{*}{ Influent $(\mu \mathrm{g} / \mathrm{l})$} & nd & \multirow{3}{*}{9} & nd & \multirow{3}{*}{$8(b)$} & nd & \multirow{3}{*}{17 (de) } \\
\hline & 0,31 & & 0,69 & & 1,5 & \\
\hline & 0,19 & & 0,42 & & 0,45 & \\
\hline Pig slurry & nd & 4 & & & & \\
\hline Warning system for pesticide leaching & nd & 9 & & & & \\
\hline \multicolumn{7}{|l|}{ to groundwater } \\
\hline Drain water & nd & 1 & & & & \\
\hline Landfill leachates & & & nd & $9^{28}(b)$ & & \\
\hline
\end{tabular}

References:

a) (Mogensen, et al., 2008)

b) (Møskeland, et al., 2006)

c) (Thomas, et al., 2007)

d) (Fick, et al., 2011)

e) (Johansson, et al., 2003)

f) (Sternbeck, et al., 2007a)

$25 \mathrm{Up}$ and downstream of fish farms

26 Includes 2 samples at proximity of hospitals

27 Samples at proximity of farms

${ }^{28}$ Includes 5 treated samples 


\section{Conclusion:}

The compound was monitored in three Swedish studies, two Norwegian and one Danish studies. The compound was detected in several matrices:

- In one biota sample in Sweden, not in any other country

- STP sludge in Sweden and Norway but not Denmark

- Effluent in all three countries

- Surface water in Sweden

- STP influent water in all three countries

\subsubsection{Tetracycline}

CAS nr.: 60-54-8

Info: Broad spectrum antibiotic

Toxicity data:

EC50 21 days (Daphnia magna) $=44.8 \mathrm{mg} / \mathrm{l}$

EC50 7 days (Microcystis aeruginosa) $=0.09 \mathrm{mg} / \mathrm{l}$

EC50 3 days (Selenastrum capricomutum) $=2.2 \mathrm{mg} / \mathrm{l}$

Nitszscha closterium EC50, 72 h 16 mg/l

EC50 96h (Salvenius namaycush) $=220 \mathrm{mg} / \mathrm{l}$

LC50 96 h (Morone saxatilis) $>182 \mathrm{mg} / \mathrm{L}$

EC50 7 days (Lemna Gibba) $=723 \mu \mathrm{g} / \mathrm{l}$

(Andersson, et al., 2006)

$P N E C=9 \mathrm{ug} / \mathrm{l}$ (Andersson, et al., 2006)

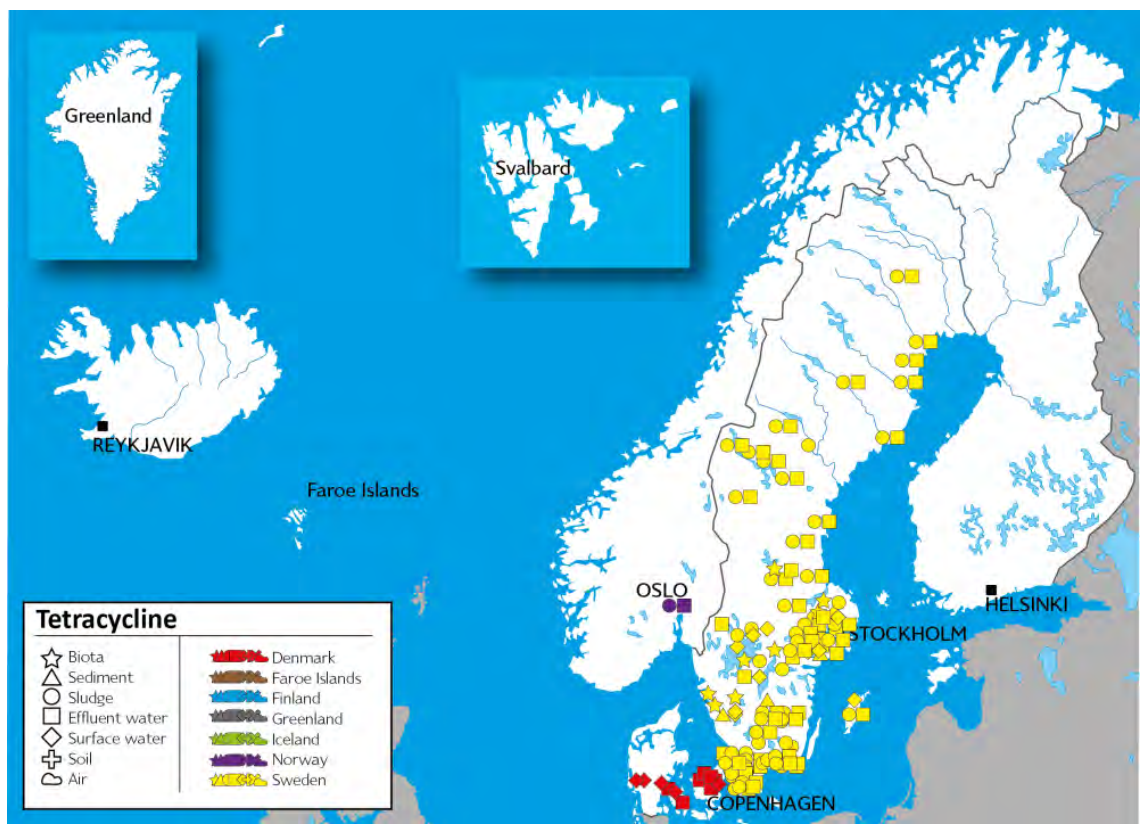




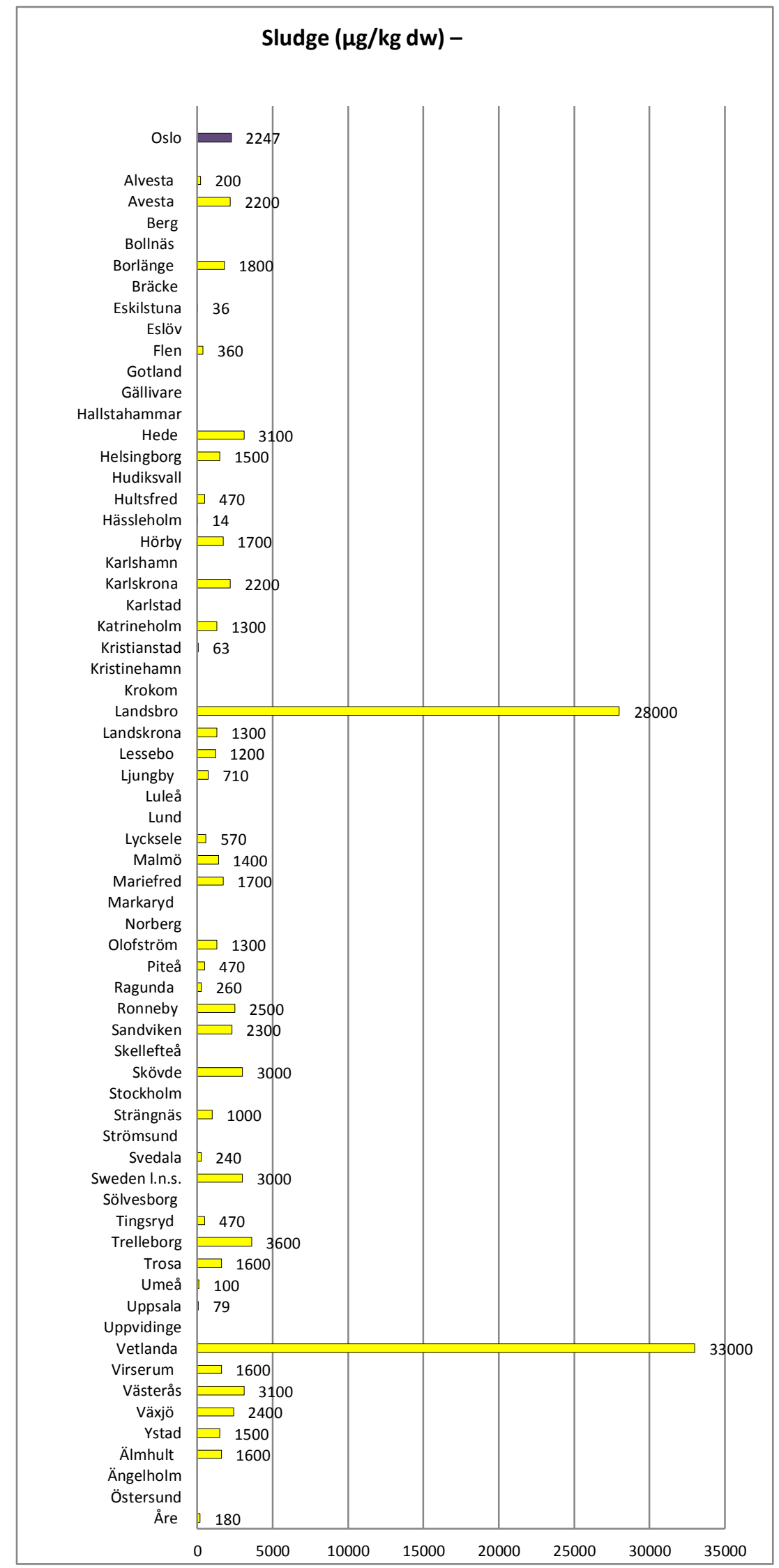




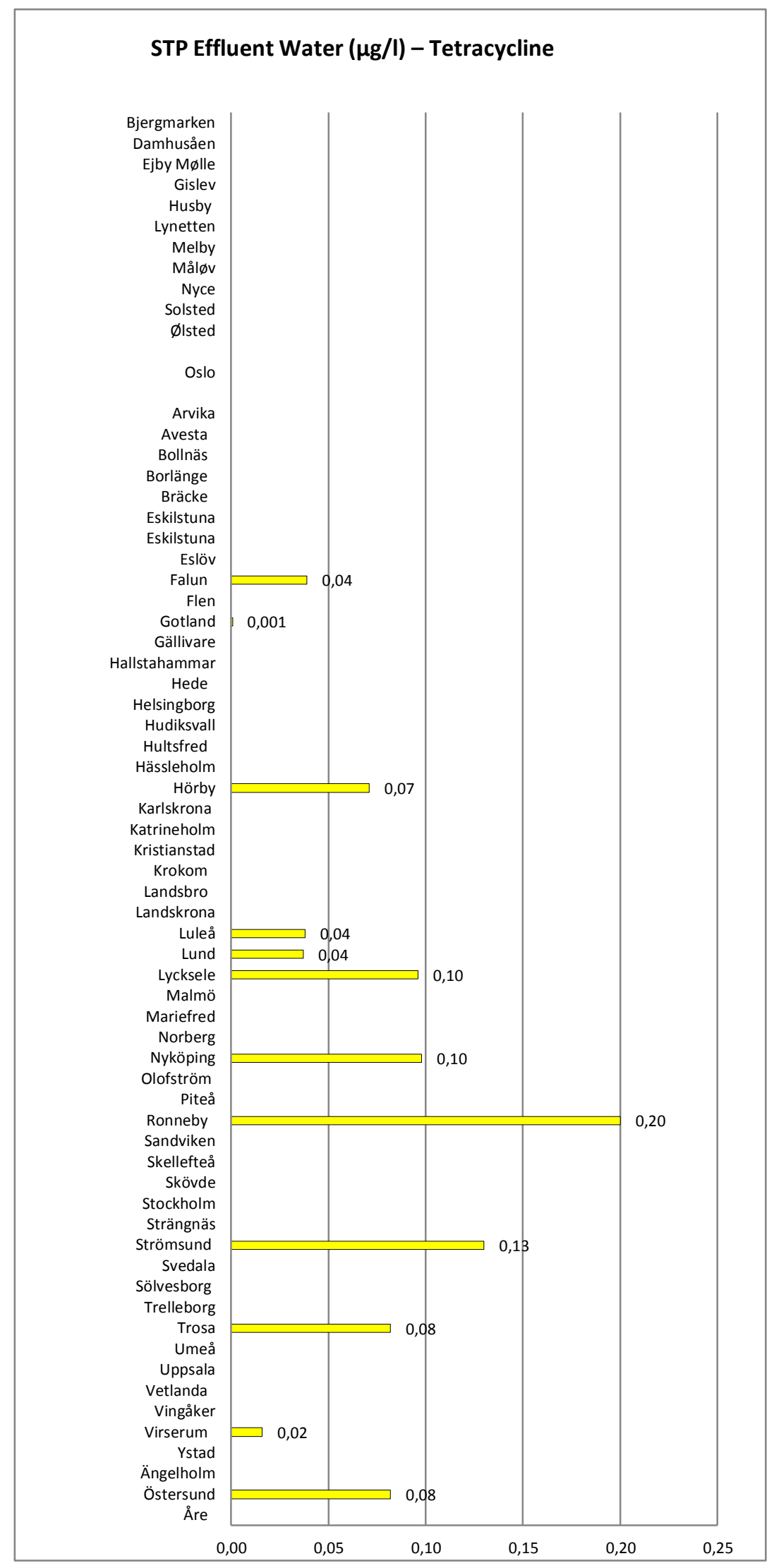




\begin{tabular}{|c|c|c|c|c|c|c|}
\hline $\begin{array}{l}\text { Tetracycline } \\
\text { Matrix }\end{array}$ & \multicolumn{2}{|c|}{ Denmark (a) } & \multicolumn{2}{|c|}{ Norway (b) } & \multicolumn{2}{|c|}{ Sweden } \\
\hline Matrix & $\begin{array}{r}\min \\
\max \\
A D\end{array}$ & $\begin{array}{l}\text { Nr.S } \\
\text { (ref) }\end{array}$ & $\begin{array}{r}\min \\
\max \\
A D\end{array}$ & $\begin{array}{l}\text { Nr.S } \\
\text { (ref) }\end{array}$ & $\begin{array}{r}\min \\
\max \\
A D\end{array}$ & $\begin{array}{l}\text { Nr.S } \\
\text { (ref) }\end{array}$ \\
\hline Biota $(\mu \mathrm{g} / \mathrm{kg} \mathrm{dw})$ & & & & & nd & $18(\mathrm{~cd})$ \\
\hline Sediment & & & & & nd & 7 (c) \\
\hline STP sludge (mg/kg dw) & & & $\begin{array}{r}0,16 \\
6,7 \\
2,2\end{array}$ & 7 & $\begin{array}{r}\text { nd } \\
33 \\
2,7\end{array}$ & $66(\mathrm{~cd})$ \\
\hline Drinking water & & & & & nd & 6 (d) \\
\hline STP effluent $(\mu \mathrm{g} / \mathrm{l})$ & nd & 9 & nd & 7 & $\begin{array}{r}\text { nd } \\
0,2 \\
0,08\end{array}$ & $68(\mathrm{~cd})$ \\
\hline Surface water $(\mu \mathrm{g} / \mathrm{l})$ & nd & 16 & & & $\begin{array}{r}\text { nd } \\
0,002 \\
0,002\end{array}$ & $32(c d)$ \\
\hline Groundwater & nd & $3^{29}$ & & & & \\
\hline Hospital WW ( $\mu \mathrm{g} / \mathrm{l})$ & & & & & $\begin{array}{r}\text { nd } \\
1400 \\
650,3\end{array}$ & 7 (c) \\
\hline Influent ( $\mu \mathrm{g} / \mathrm{l})$ & $\begin{array}{r}\text { nd } \\
0,34 \\
0,25\end{array}$ & 9 & & & $\begin{array}{r}\text { nd } \\
1800 \\
310\end{array}$ & $31(\mathrm{~cd})$ \\
\hline Pig slurry & nd & 4 & & & & \\
\hline $\begin{array}{l}\text { Warning system for pesticide leaching to } \\
\text { groundwater }(\mathrm{mg} / \mathrm{kg} \mathrm{dw})\end{array}$ & $\begin{array}{r}\text { nd } \\
0,18 \\
0,18\end{array}$ & 9 & & & & \\
\hline Drain water & nd & 1 & & & & \\
\hline Manure $(\mu \mathrm{g} / \mathrm{kg} \mathrm{dw})$ & & & & & $\begin{array}{r}\text { nd } \\
400 \\
400\end{array}$ & 5 (c) \\
\hline Landfill leachates water $(\mu \mathrm{g} / \mathrm{l})$ & & & & & $\begin{array}{r}\text { nd } \\
0,03 \\
0,03\end{array}$ & 8 (c) \\
\hline
\end{tabular}

\section{References:}

a) (Mogensen, et al., 2008)

b) (Thomas, et al., 2007)

c) (Andersson, et al., 2006)

d) (Fick, et al., 2011)

\section{Conclusion:}

The compound was monitored in one Danish, one Norwegian and two Swedish studies. The compound was detected in several matrices:

${ }^{29}$ Includes 2 samples at proximity of hospitals 
- STP sludge in Norway and Sweden. Very high concentrations were detected in two locations in Sweden

- STP effluent in Sweden, in concentrations below the PNEC value. It was not detected in Norway or Denmark

- In one sample of surface water in Sweden, the value detected was below the PNEC

- In one sample of manure in Sweden

- In one sample of landfill leachates in Sweden, the value detected was below the PNEC

- Hospital waste water in Sweden, in high concentrations, well above the PNEC

- STP influent in Sweden and Denmark, in much higher concentrations in Sweden. Values detected in Sweden were above the PNEC, and below the PNEC in Denmark

\subsubsection{Triclosan}

CAS nr.: 3380-34-5

Info: A polychloro phenoxy phenol used as an antibacterial and antifungal agent

Toxicity data: NA

PNEC $=$

PNECaqua $=0,05 \mu \mathrm{g} / \mathrm{l}$ (calculation)

PNECsediment = ca $1 \mathrm{mg} / \mathrm{kg}$ (calculation)

(Sternbeck, et al., 2007a)

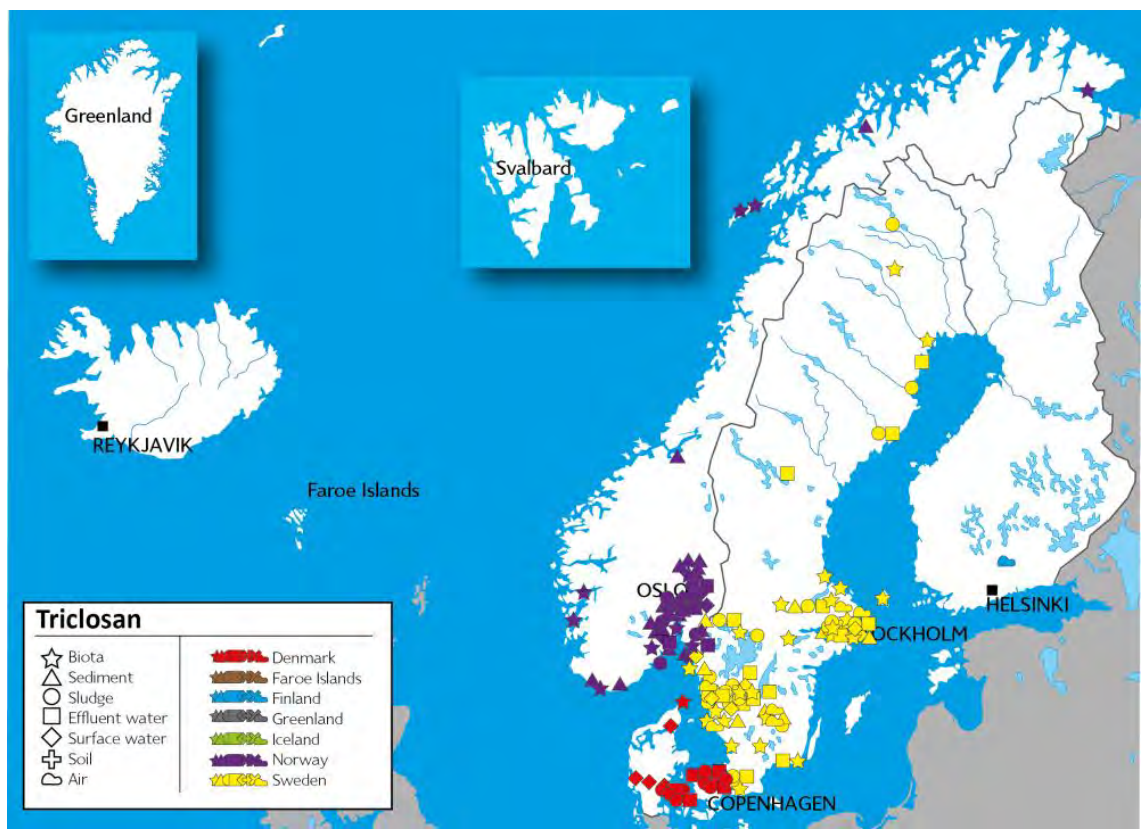




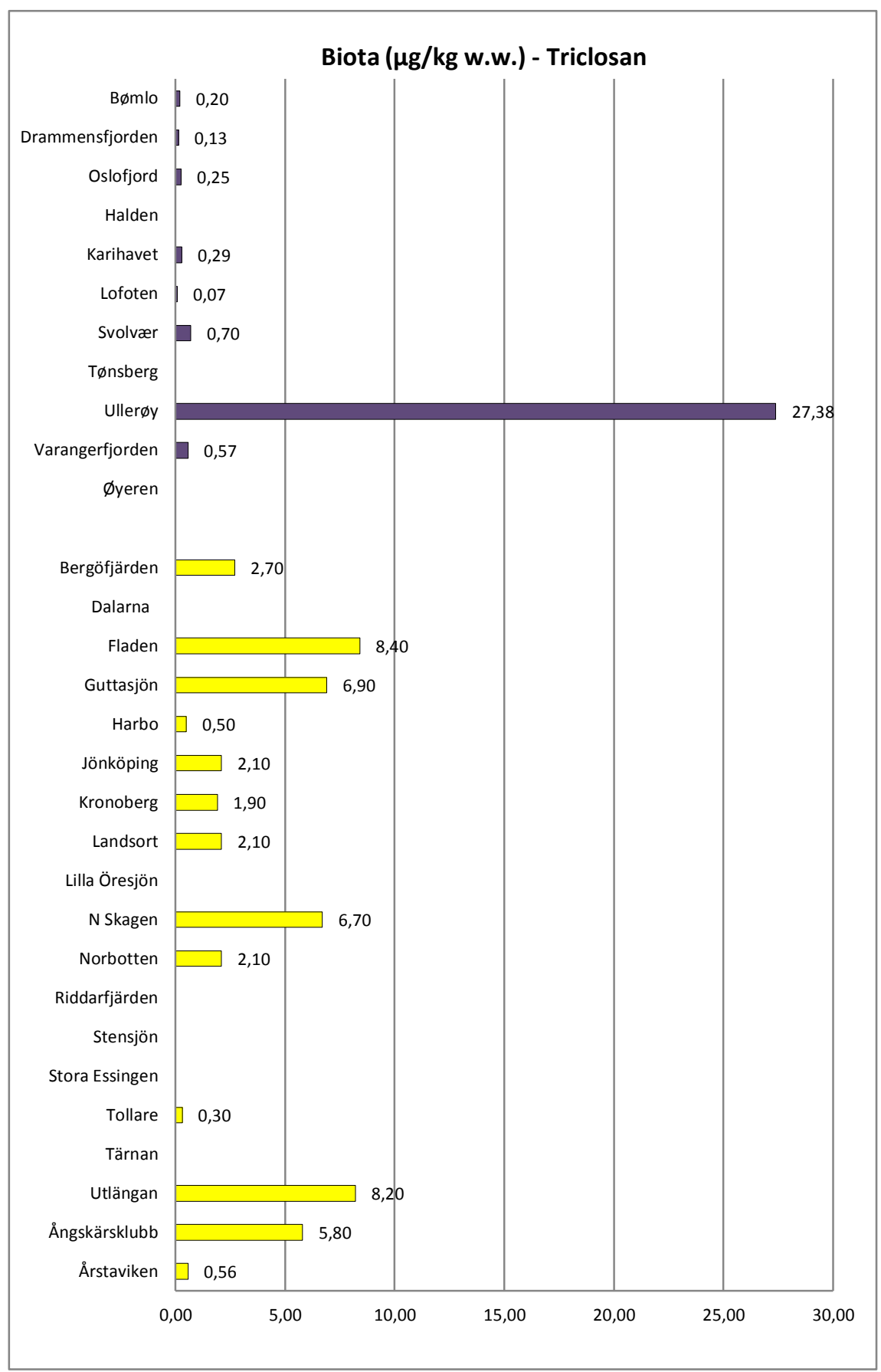




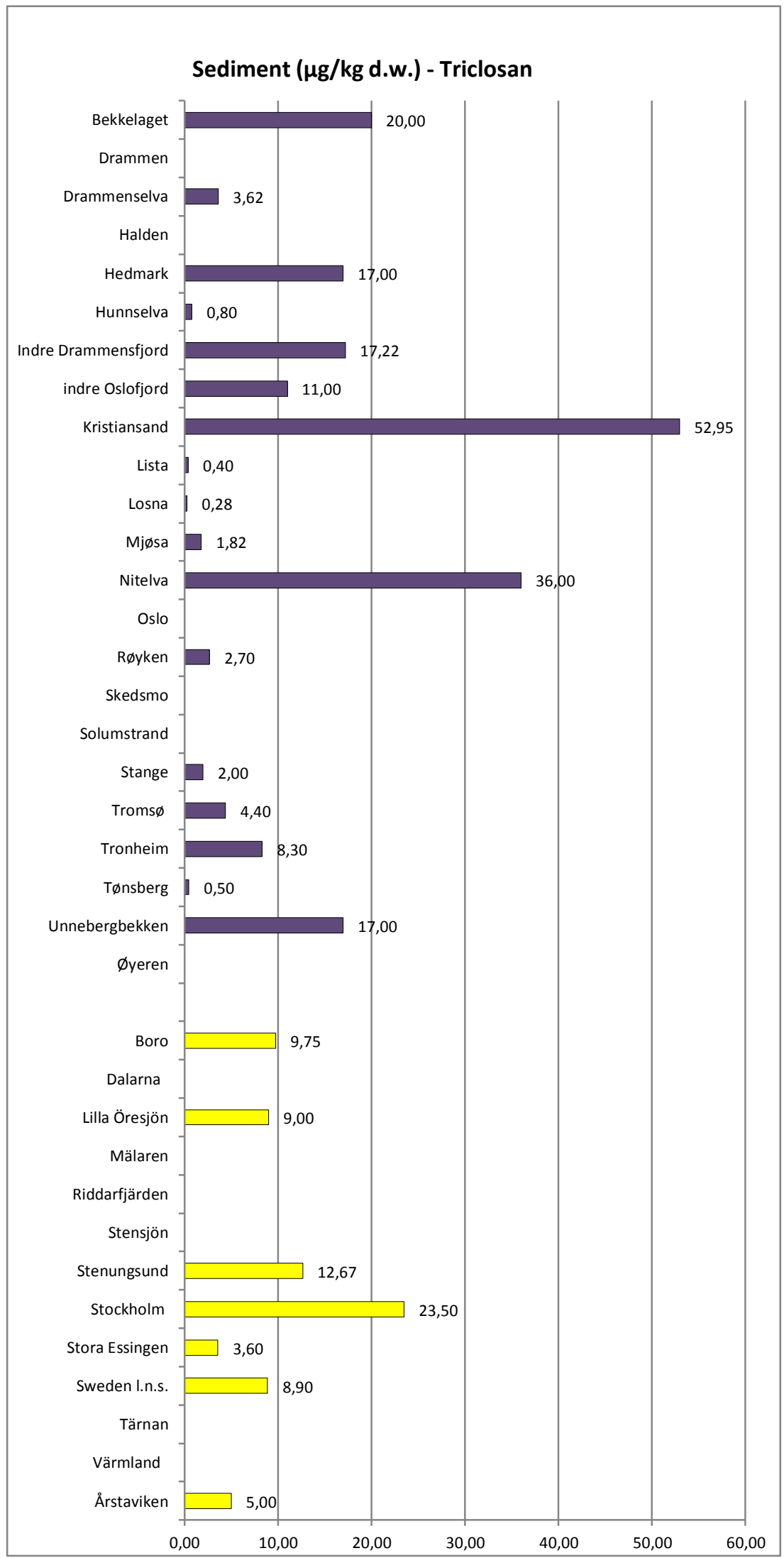




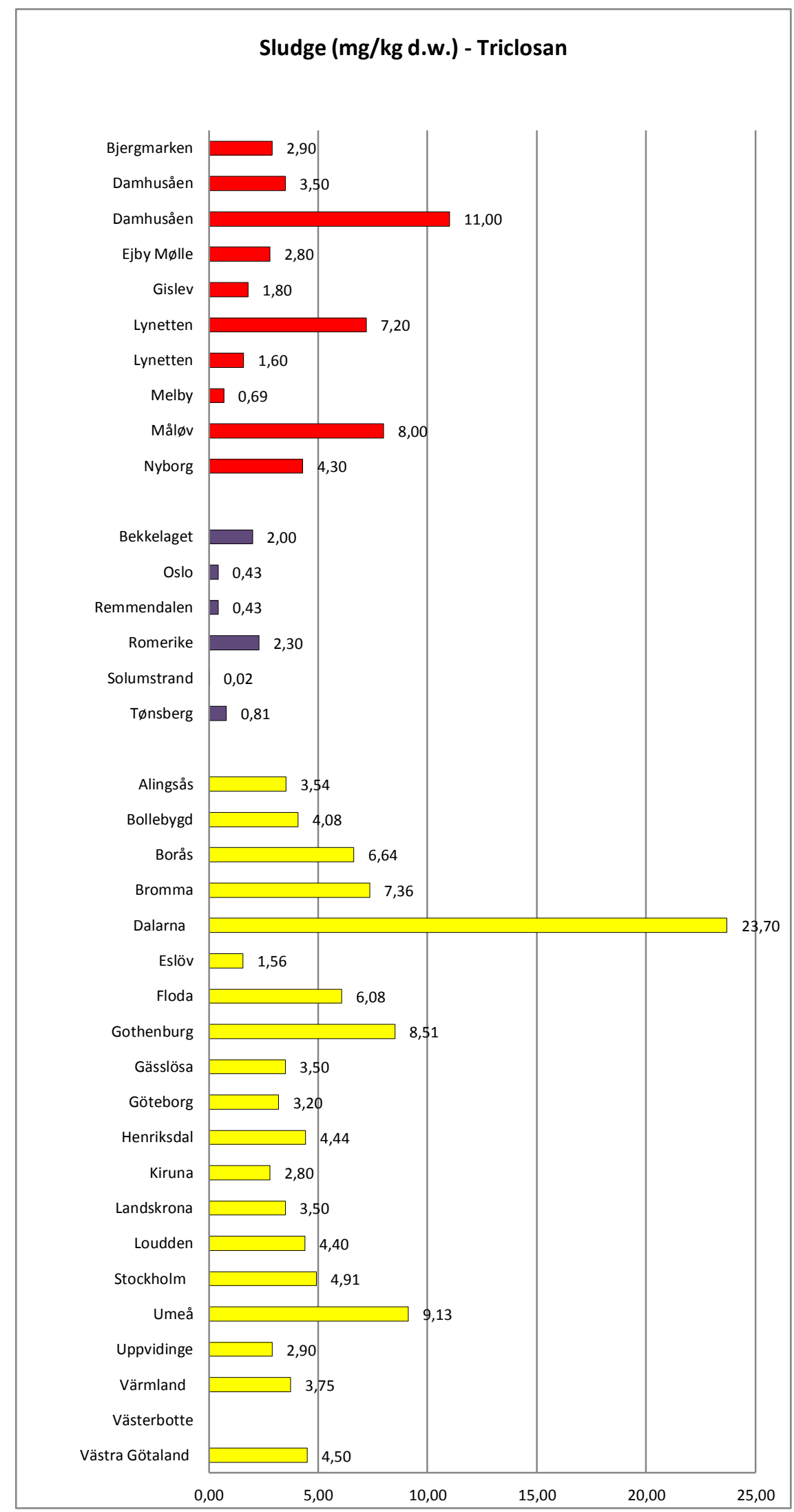




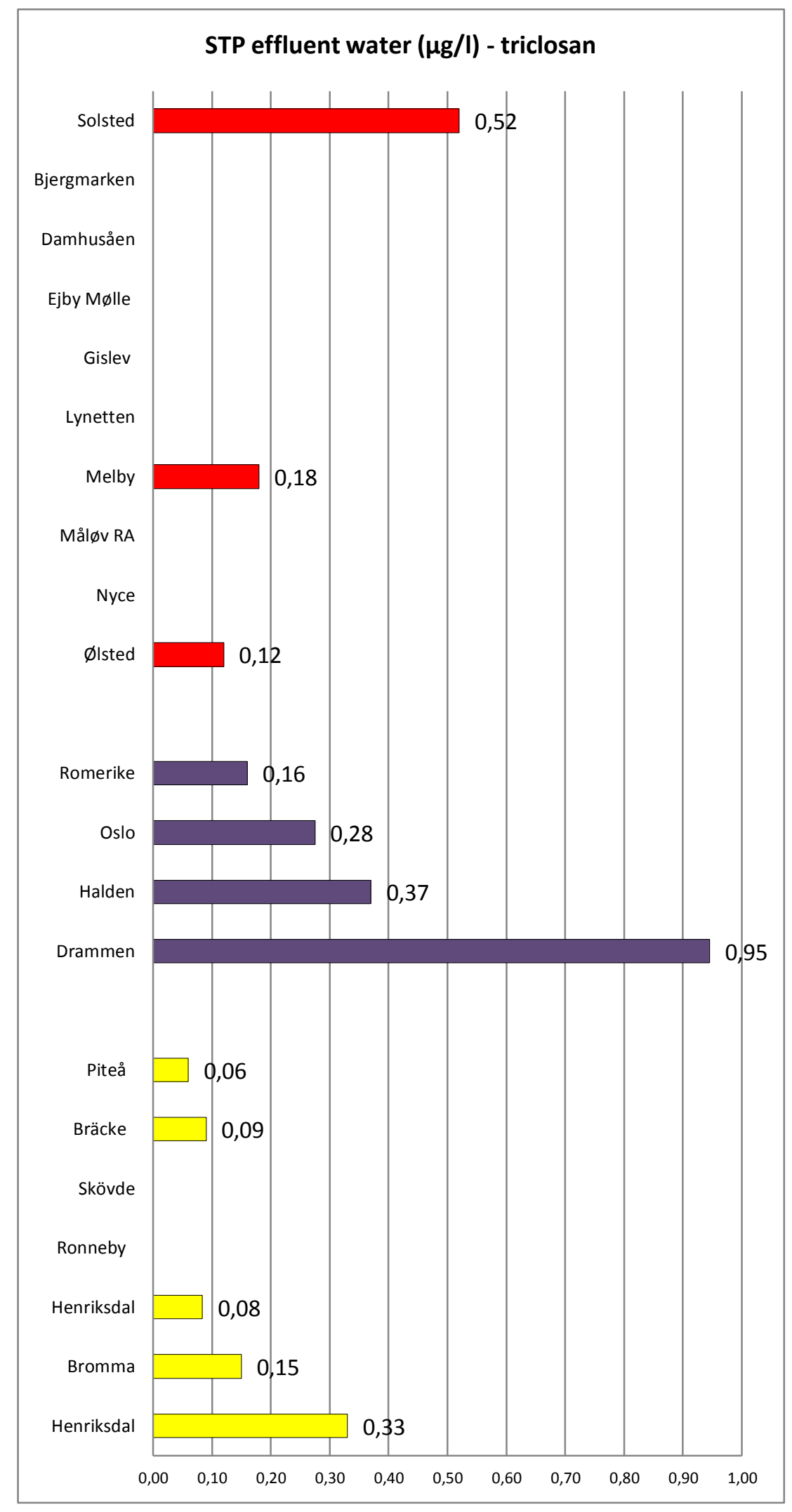




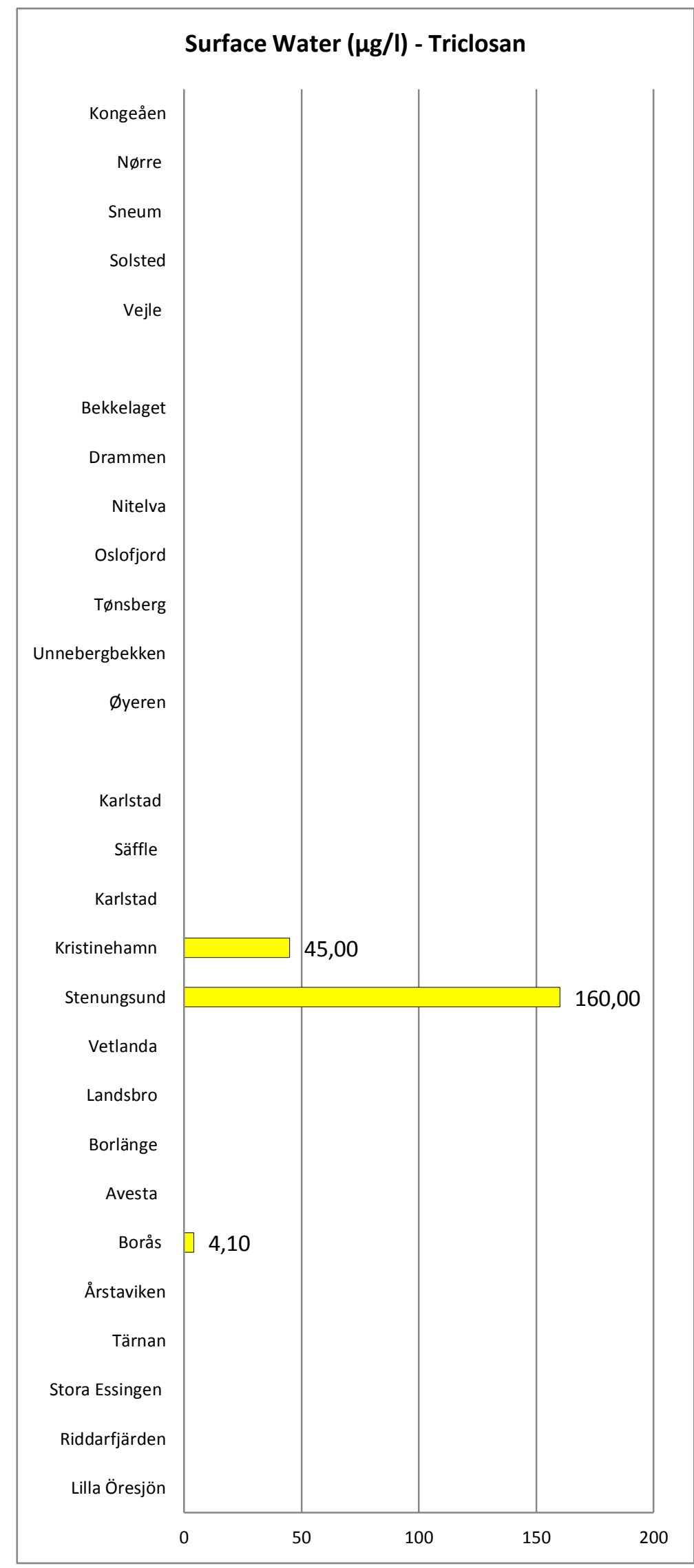




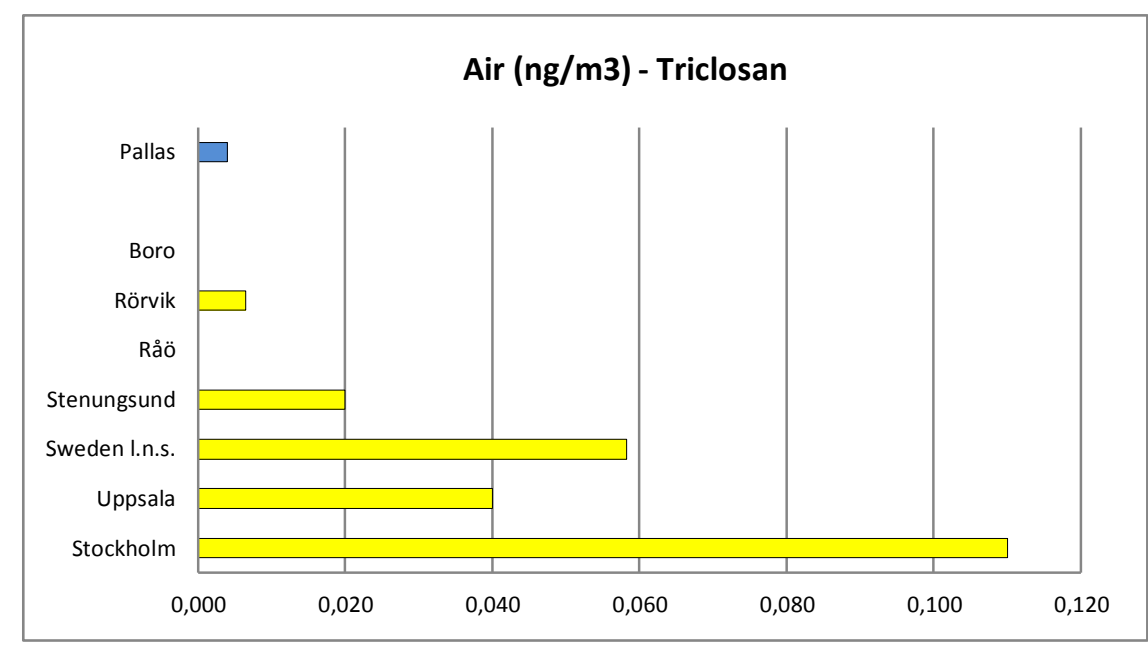

\begin{tabular}{|c|c|c|c|c|c|c|}
\hline \multirow{2}{*}{$\begin{array}{l}\text { Triclosan } \\
\text { Matrix }\end{array}$} & \multicolumn{2}{|c|}{ Denmark (a) } & \multicolumn{2}{|c|}{ Norway } & \multicolumn{2}{|c|}{ Sweden } \\
\hline & $\begin{array}{r}\min \\
\max \\
\mathrm{AD}\end{array}$ & $\begin{array}{l}\text { Nr.S } \\
\text { (ref) }\end{array}$ & $\begin{array}{r}\min \\
\max \\
A D\end{array}$ & $\begin{array}{l}\text { Nr.S } \\
\text { (ref) }\end{array}$ & $\begin{array}{r}\min \\
\max \\
A D\end{array}$ & $\begin{array}{l}\text { Nr.S } \\
\text { (ref) }\end{array}$ \\
\hline Air (ng/m3) & & & & & $\begin{array}{r}\text { nd } \\
0,17 \\
0,04\end{array}$ & 29 (def) \\
\hline Biota $(\mu \mathrm{g} / \mathrm{kg} w w)$ & & & $\begin{array}{r}\text { nd } \\
27,4 \\
3,3\end{array}$ & 21 (bc) & $\begin{array}{r}\text { nd } \\
13 \\
4,2\end{array}$ & 21 (de) \\
\hline Biota $(\mu \mathrm{g} / \mathrm{kg} \mathrm{dw})$ & & & $\begin{array}{r}\text { nd } \\
0,37 \\
0,1\end{array}$ & 12 (c) & & \\
\hline Biota (ng/g fw) & & & & & $\begin{array}{l}\text { nd } \\
6,9 \\
4,3\end{array}$ & 7 (ef) \\
\hline Sediment $(\mu \mathrm{g} / \mathrm{kg} \mathrm{dw})$ & & & $\begin{array}{r}\text { nd } \\
105 \\
11,7\end{array}$ & 41 (bc) & $\begin{array}{l}\text { nd } \\
25 \\
9,3\end{array}$ & 22 (def) \\
\hline STP sludge (mg/kg dw) & $\begin{array}{r}0,69 \\
11 \\
4,4\end{array}$ & 10 & $\begin{array}{r}\text { nd } \\
23 \\
0,9\end{array}$ & 6 (b) & $\begin{array}{r}\text { nd } \\
35 \\
5,3\end{array}$ & 68 (defghij) \\
\hline STP effluent $(\mu \mathrm{g} / \mathrm{l})$ & $\begin{array}{r}\text { nd } \\
0,52 \\
0,27\end{array}$ & 7 & $\begin{array}{l}0,16 \\
0,94 \\
0,44\end{array}$ & 4 (b) & $\begin{array}{r}\text { nd } \\
0,33 \\
0,14\end{array}$ & 7 (ej) \\
\hline Surface water $(\mu \mathrm{g} / \mathrm{l})$ & nd & 16 & nd & 8 (b) & $\begin{array}{r}\text { nd } \\
0,16 \\
0,07\end{array}$ & 34 (def) \\
\hline Groundwater & nd & 3 & & & & \\
\hline Soil ( $\mu \mathrm{g} / \mathrm{kg} \mathrm{dw})$ & & & & & $\begin{array}{r}\text { nd } \\
15 \\
9,5\end{array}$ & 7 (d) \\
\hline Influent ( $\mu \mathrm{g} / \mathrm{l})$ & $\begin{array}{r}\text { nd } \\
1,4 \\
0,71\end{array}$ & 9 & $\begin{array}{r}0,13 \\
3,9 \\
1\end{array}$ & 8 (b) & $\begin{array}{l}0,6 \\
0,6 \\
0,6\end{array}$ & 1 (e) \\
\hline
\end{tabular}




\begin{tabular}{|c|c|c|c|c|c|c|}
\hline \multirow{2}{*}{$\begin{array}{l}\text { Triclosan } \\
\text { Pig slurry }\end{array}$} & \multicolumn{2}{|c|}{ Denmark (a) } & \multicolumn{2}{|c|}{ Norway } & \multicolumn{2}{|c|}{ Sweden } \\
\hline & nd & 4 & & & & \\
\hline Deposition (ng/m2/day) & & & & & $\begin{array}{l}\text { nd } \\
20 \\
5,9\end{array}$ & 11 (def) \\
\hline Food ( $\mu \mathrm{g} / \mathrm{kg} w w)$ & & & & & $\begin{array}{l}\text { nd } \\
8,4 \\
4,5\end{array}$ & $18(d)$ \\
\hline Landfill leachates sediment ( $\mu \mathrm{g} / \mathrm{kg} \mathrm{dw})$ & & & $\begin{array}{l}\text { nd } \\
63 \\
63\end{array}$ & 4 (b) & & \\
\hline Landfill leachates water & & & nd & 9 (b) & nd & $3^{30}(\mathrm{e})$ \\
\hline $\begin{array}{l}\text { Warning system for pesticide leaching to } \\
\text { groundwater }\end{array}$ & nd & 6 & & & & \\
\hline Drain water & nd & 1 & & & & \\
\hline Precipitation $(\mu \mathrm{g} / \mathrm{l})$ & & & & & nd & 3 (e) \\
\hline
\end{tabular}

\section{References:}

a) (Mogensen, et al., 2008)

b) (Møskeland, et al., 2006)

c) (Fjeld, et al., 2006)

d) (Remberger, et al., 2002)

e) (Remberger, et al., 2006)

f) (Sternbeck, et al., $2007 \mathrm{~b}$ )

g) (Haglund \& Olofsson, 2006)

h) (Haglund \& Olofsson, 2007)

i) (Haglund \& Olofsson, 2008)

j) (Adolfsson-Erici, et al., 2003)

\section{Conclusion:}

The compound was monitored in one study in Denmark, two in Norway and seven throughout Sweden. It was detected in several matrices:

- Air samples from Sweden

- Biota in Norway and Sweden

- Sediment in Norway and Sweden, in concentrations below the PNEC

- STP sludge in all three countries. Higher values were observed in Norway

- STP effluent in all three countries, with average detected values above the PNEC

- Surface water only in Sweden, in concentrations above the PNEC. The compound was not detected in Finland or Norway

30 Includes one sampe after treatment 
- Soil in Sweden

- Air deposition samples in Sweden

- Food samples from Sweden

- Sediment leachates from landfills in Norway, values were below the PNEC

- STP influent in all three countries

\subsubsection{Trimethoprim}

CAS nr. 738-70-5

Info: $A$ bacteriostatic antibiotic

Toxicity data:

LOEC 7 days (Lemna gibba) $>1000 \mu \mathrm{g} / \mathrm{l}$

EC50 (cyanobacteria) $>200000 \mu \mathrm{g} / \mathrm{l}$

EC50 (R. salina) $=160 \mu \mathrm{g} / \mathrm{l}$

(Sternbeck, et al., 2007a)

NOEC 7 days (Lemna gibba) $=1 \mathrm{mg} / \mathrm{l}$

EC50 (M. aeruginosa) $=112 \mathrm{mg} / \mathrm{l}$

EC50 (S. Capricornutum) $=130 \mathrm{mg} / \mathrm{l}$

(Hellström \& Kreuger, 2005)

$P N E C=N A$

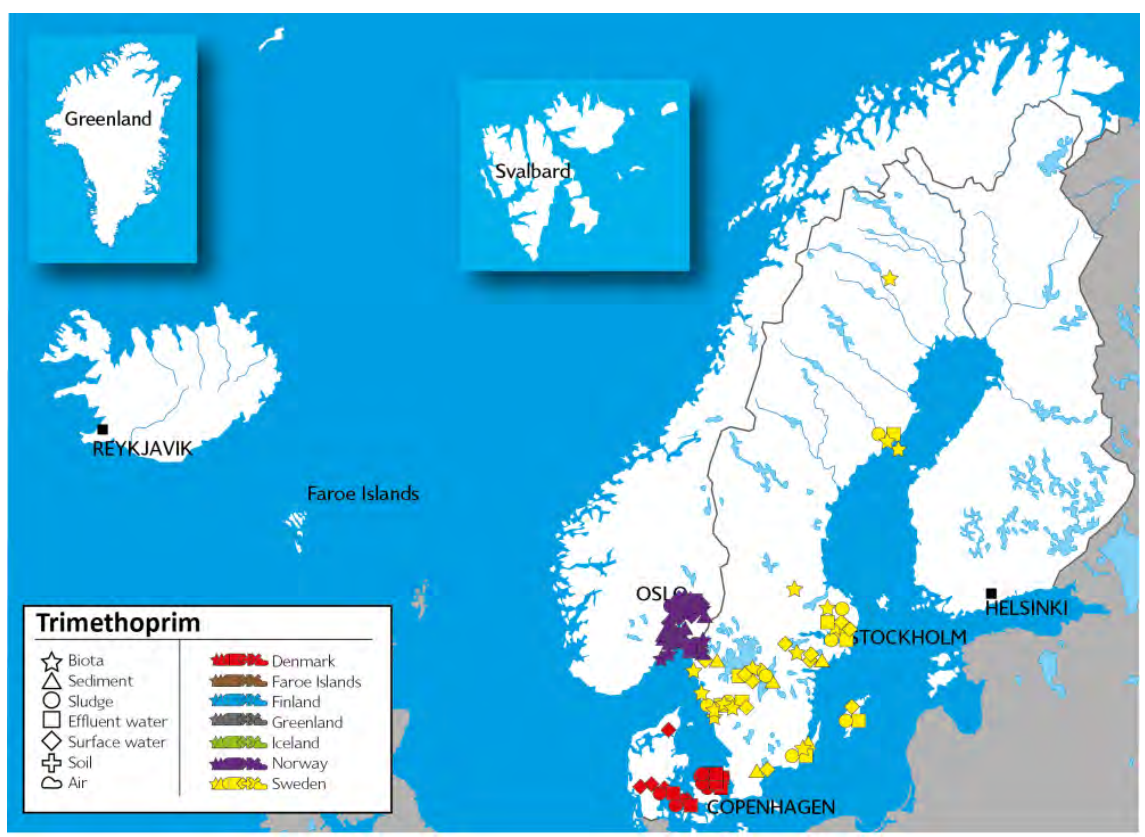




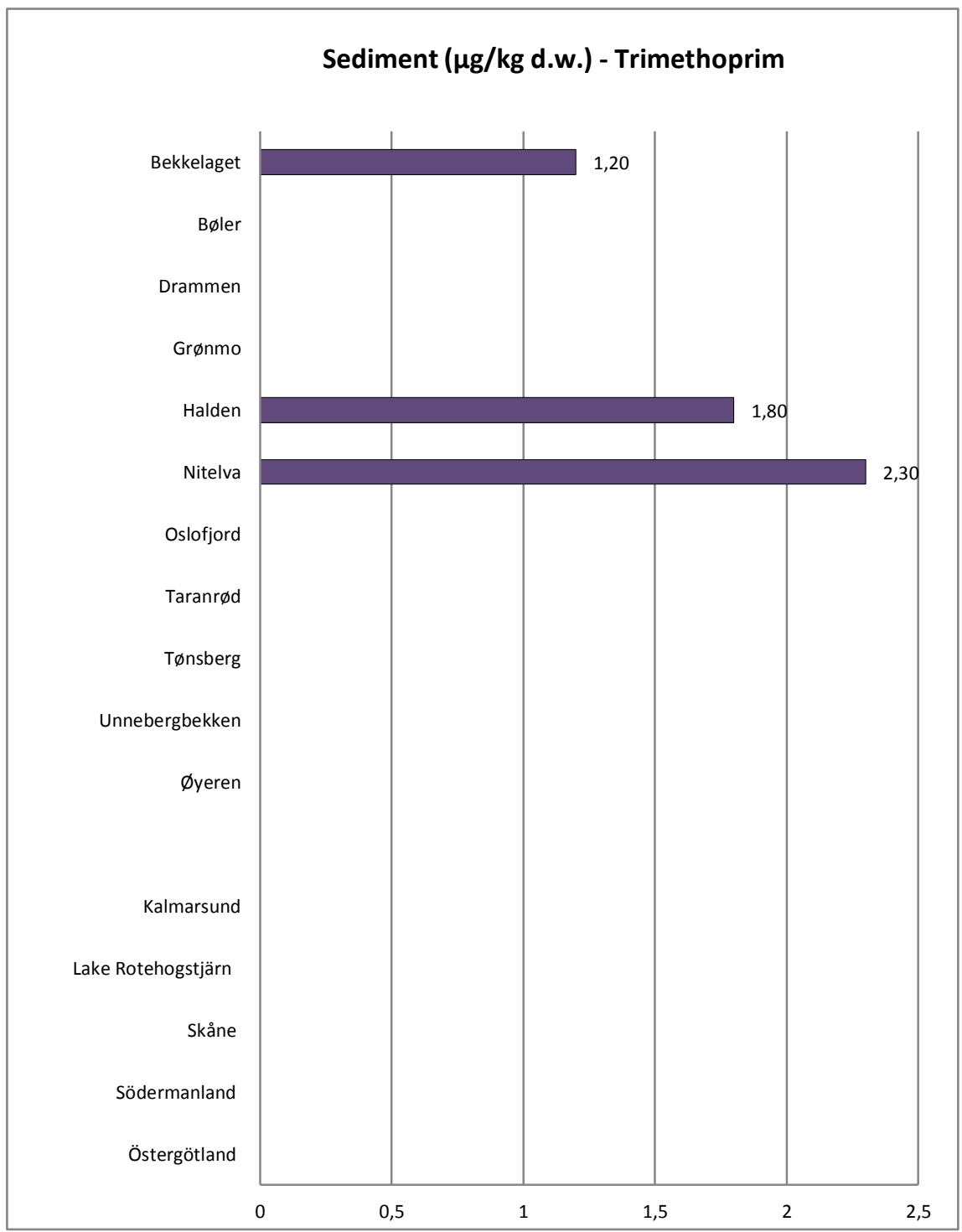




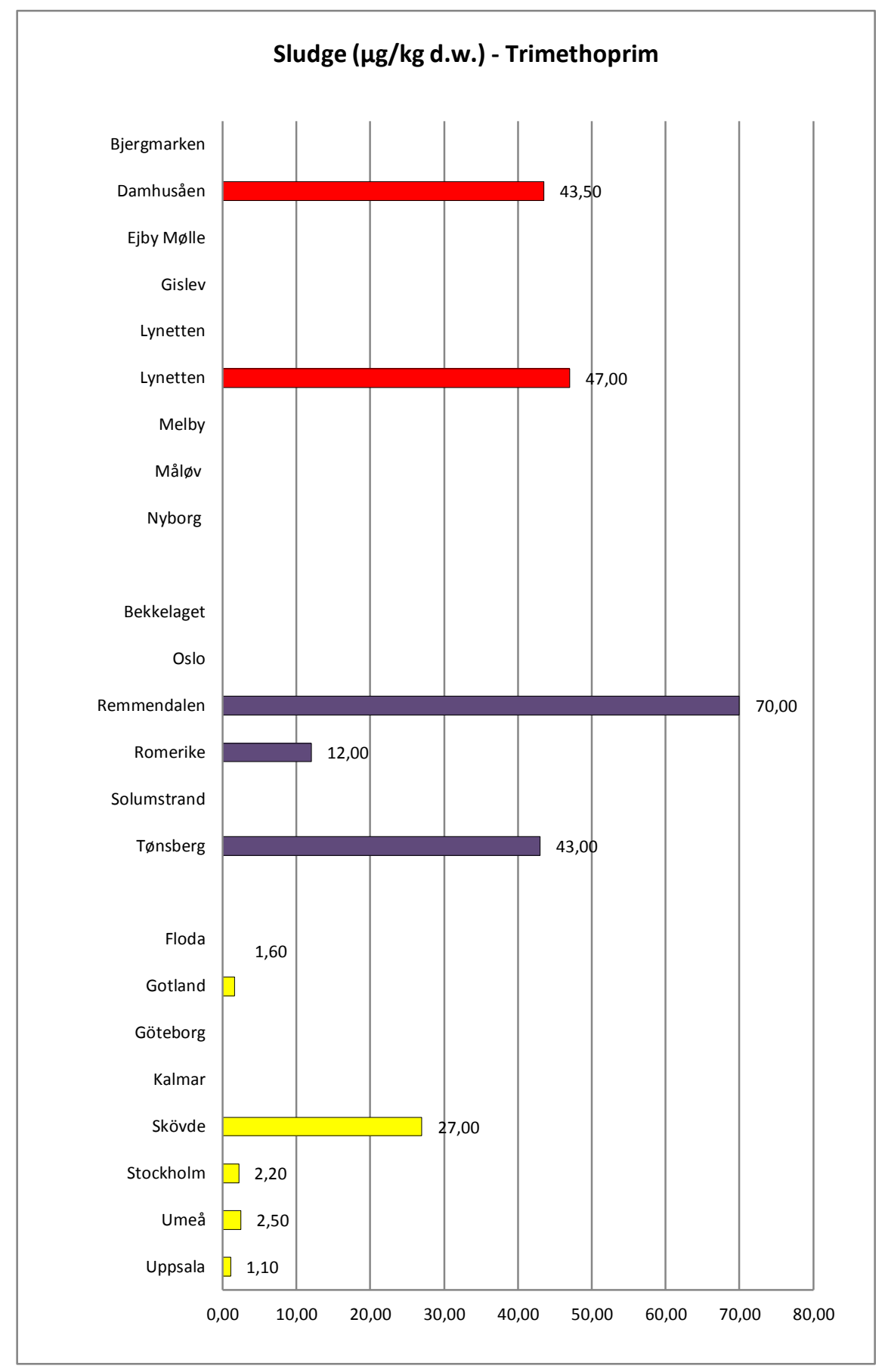




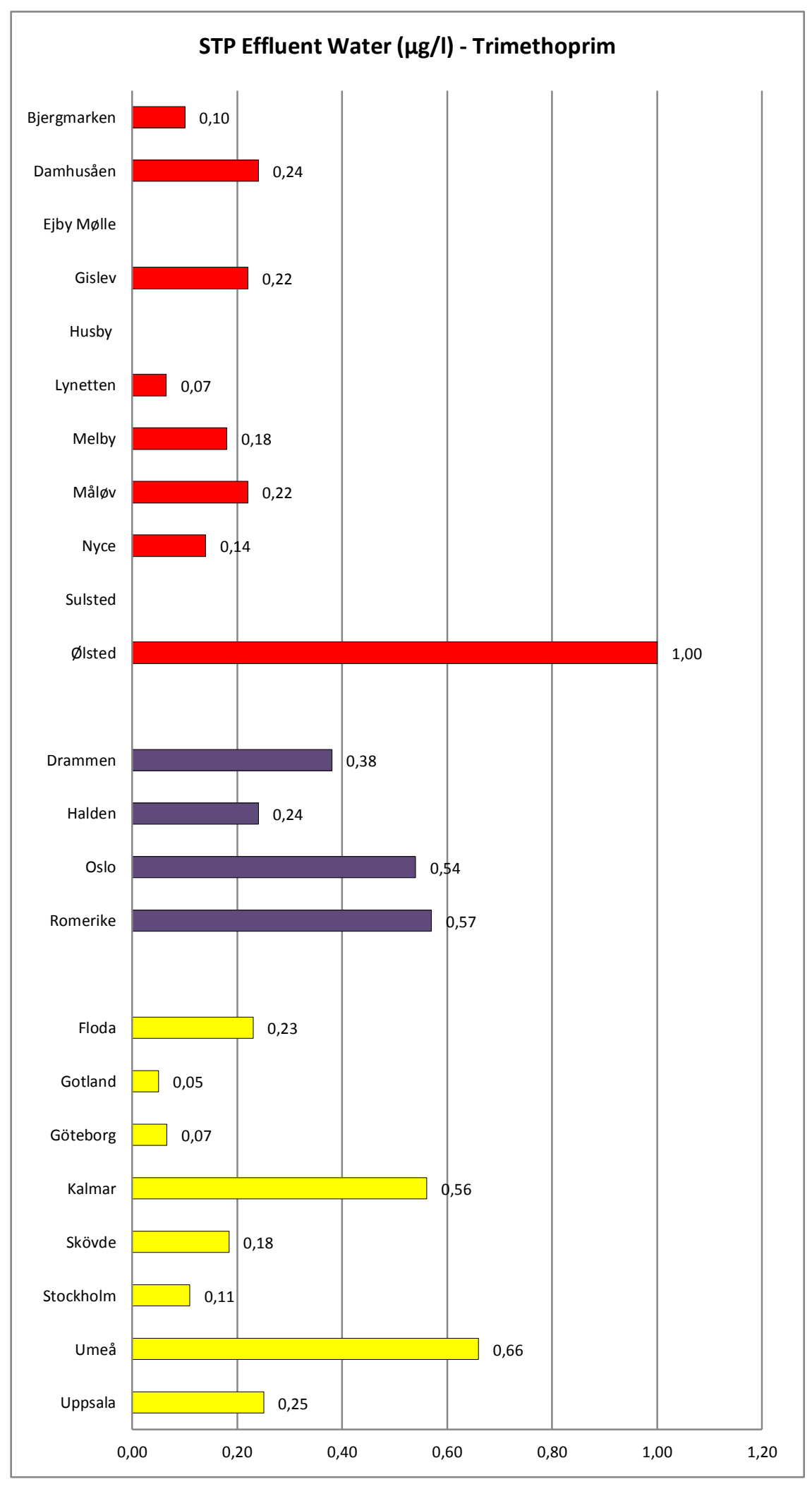




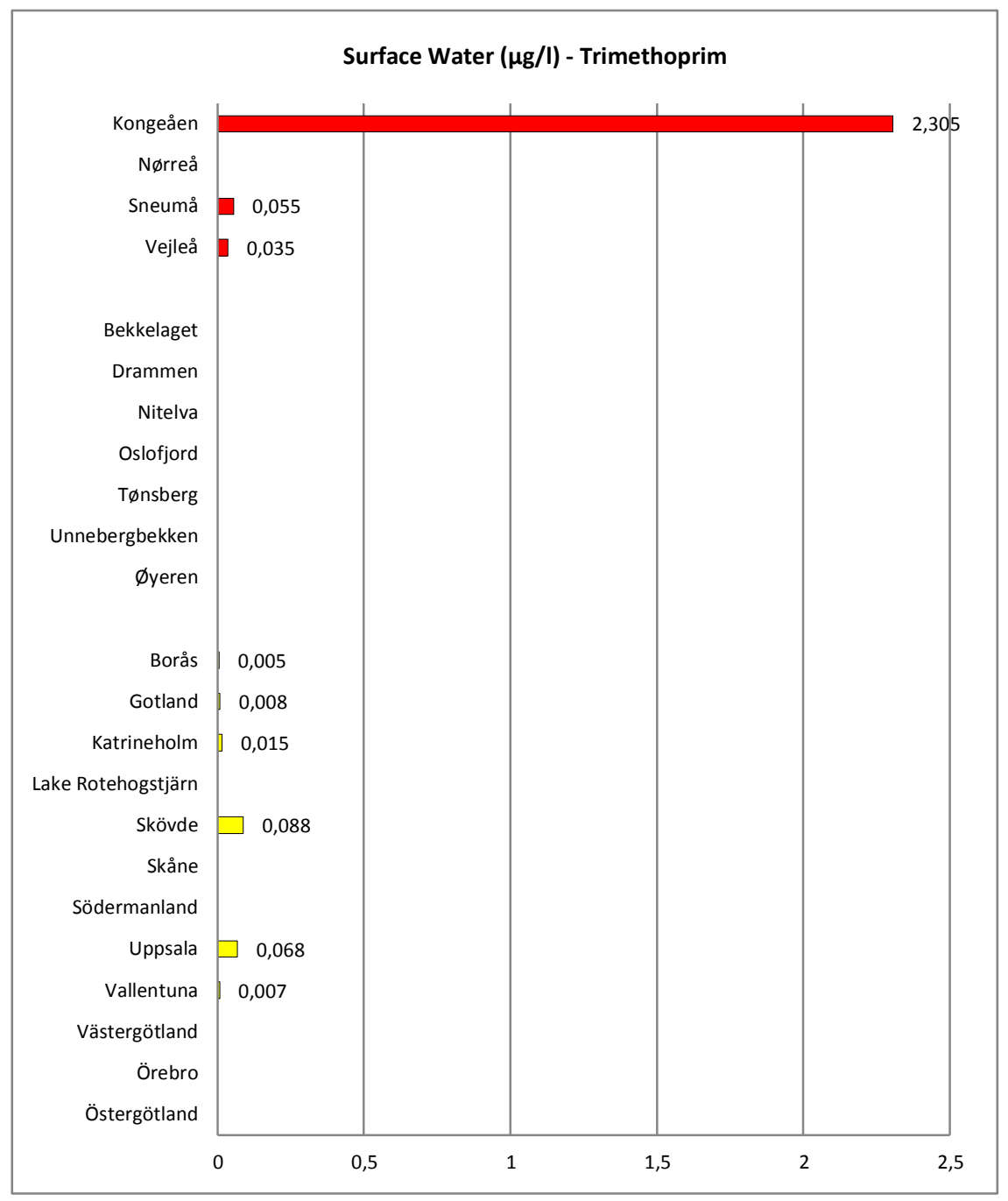

\begin{tabular}{|c|c|c|c|c|c|c|}
\hline \multirow{2}{*}{$\begin{array}{l}\text { Trimethoprim } \\
\text { Matrix }\end{array}$} & \multicolumn{2}{|c|}{ Denmark (a) } & \multirow{2}{*}{$\begin{array}{r}\text { Norway } \\
\min \\
\max \\
\text { AD }\end{array}$} & \multicolumn{3}{|c|}{ Sweden } \\
\hline & $\begin{array}{r}\min \\
\max \\
A D\end{array}$ & $\begin{array}{l}\text { Nr.S } \\
\text { (ref) }\end{array}$ & & $\begin{array}{l}\text { Nr.S } \\
\text { (ref) }\end{array}$ & $\begin{array}{r}\min \\
\max \\
A D\end{array}$ & $\begin{array}{l}\text { Nr.S } \\
\text { (ref) }\end{array}$ \\
\hline Biota & & & nd & $6(b)$ & nd & 24 (de) \\
\hline STP sludge ( $\mu \mathrm{g} / \mathrm{kg} \mathrm{dw})$ & $\begin{array}{r}\text { nd } \\
76 \\
44,7\end{array}$ & 10 & $\begin{array}{l}\text { nd } \\
43 \\
43\end{array}$ & $13(\mathrm{bc})$ & $\begin{array}{l}\text { nd } \\
27 \\
6,9\end{array}$ & 30 (def) \\
\hline Sediment & & & nd & $12(b)$ & & \\
\hline Drinking water $(\mu \mathrm{g} / \mathrm{l})$ & & & & & $\begin{array}{l}0,002 \\
0,007 \\
0,005\end{array}$ & 6 (e) \\
\hline
\end{tabular}




\begin{tabular}{|c|c|c|c|c|c|c|}
\hline \multirow{2}{*}{$\frac{\text { Trimethoprim }}{\text { STP effluent }(\mu \mathrm{g} / \mathrm{l})}$} & \multicolumn{2}{|c|}{ Denmark (a) } & \multirow{2}{*}{$\begin{array}{r}\text { Norway } \\
\text { nd }\end{array}$} & \multicolumn{3}{|c|}{ Sweden } \\
\hline & nd & 9 & & 11 (bc) & 0,05 & 19 (deg) \\
\hline & 1 & & 1,26 & & 1,4 & \\
\hline & 0,27 & & 0,59 & & 0,32 & \\
\hline \multirow[t]{3}{*}{ Surface water $(\mu \mathrm{g} / \mathrm{l})$} & nd & $16^{31}$ & nd & 8 (b) & nd & 45 (ef) \\
\hline & 10 & & & & 0,21 & \\
\hline & 1,18 & & & & 0,06 & \\
\hline \multirow[t]{3}{*}{ STP Influent $(\mu \mathrm{g} / \mathrm{l})$} & 0,13 & 9 & nd & (b) & 0,15 & 18 (deg) \\
\hline & 4,4 & & 1,44 & & 1,4 & \\
\hline & 0,74 & & 0,69 & & 0,43 & \\
\hline Groundwater & nd & $3^{32}$ & & & nd & $2(f)$ \\
\hline Farm soil & & & & & nd & $6(f)$ \\
\hline Manure & nd & $4^{33}$ & & & nd & $6(f)$ \\
\hline \multirow[t]{3}{*}{ Hospital wastewater $(\mu \mathrm{g} / \mathrm{l})$} & & & & & nd & \\
\hline & & & & & 4,1 & 2 (d) \\
\hline & & & & & 4,1 & $2(a)$ \\
\hline $\begin{array}{l}\text { Warning system for pesticide leaching to } \\
\text { groundwater }\end{array}$ & nd & 9 & & & & \\
\hline Drain water & nd & 1 & & & & \\
\hline Landfill leachates, water & & & nd & $9^{34}(b)$ & & \\
\hline Landfill leachates, sediment & & & nd & 4 (b) & & \\
\hline
\end{tabular}

\section{References:}

a) (Mogensen, et al., 2008)

b) (Møskeland, et al., 2006)

c) (Thomas, et al., 2007)

d) (Johansson, et al., 2003)

e) (Fick, et al., 2011)

f) (Sternbeck, et al., 2007a)

g) (Hellström \& Kreuger, 2005)

\section{Conclusion:}

The compound was monitored in one study in Denmark, two in Norway and four in Sweden. It was detected in several matrices:

- Surface water in Denmark and Sweden, not in Norway

- STP sludge in Denmark and Sweden and in one sample in Norway. The values detected in Sweden were lower than in the other countries

\footnotetext{
$31 \mathrm{Up}$ and downstream of fish farms

322 samples at proximity of hospitals

33 Pig slurry

34 Includes 5 treated samples
} 
- STP effluent in all three countries

- Drinking water in Sweden

- In one hospital waste water sample in Sweden

- STP influent in all three countries

\subsubsection{Cetrimonium salts}

CAS nr.: 112-02-7/ 57-09-0

Info: Quaternary ammonium salts or quaternary ammonium compounds used as antiseptic

Toxicity data: LOEC (Microcystis sp.) $=25000 \mathrm{ng} / \mathrm{L}$

$P N E C=N A$

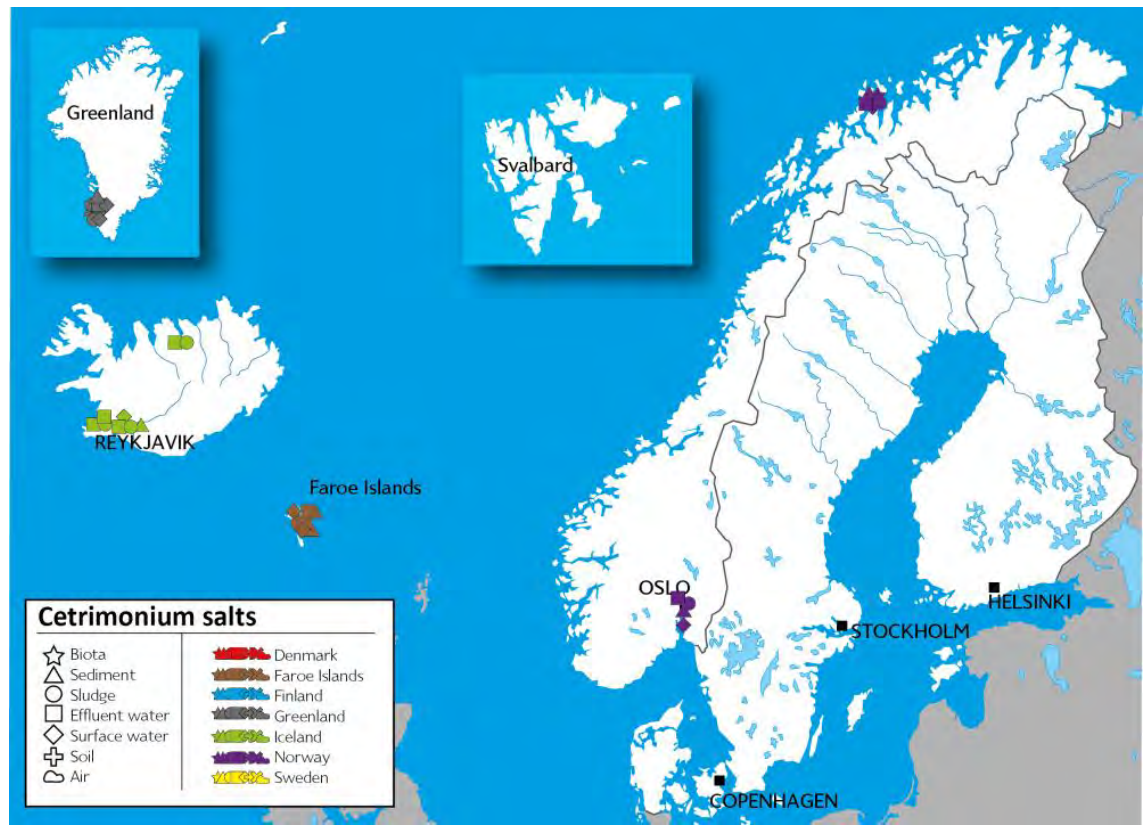



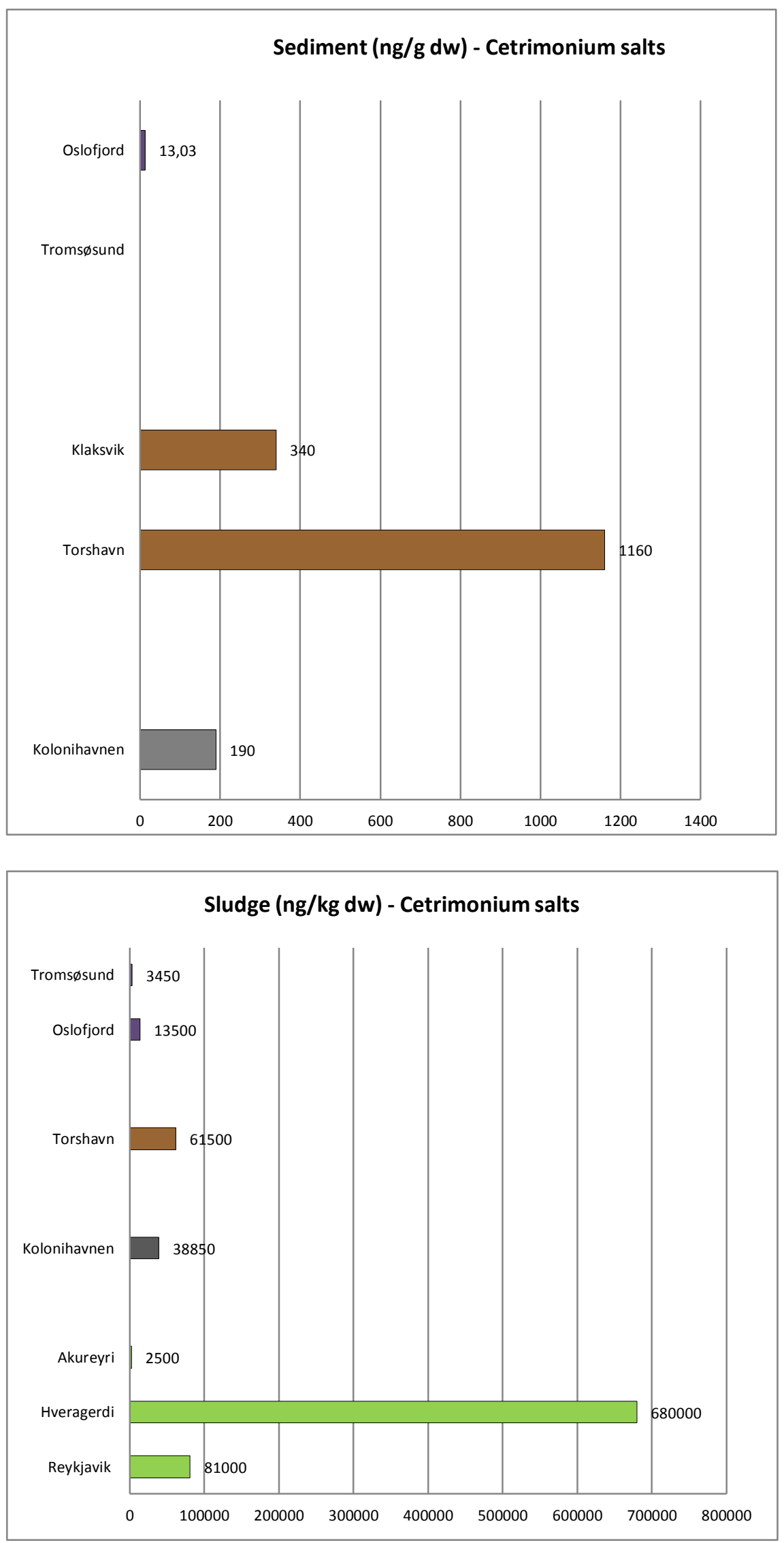

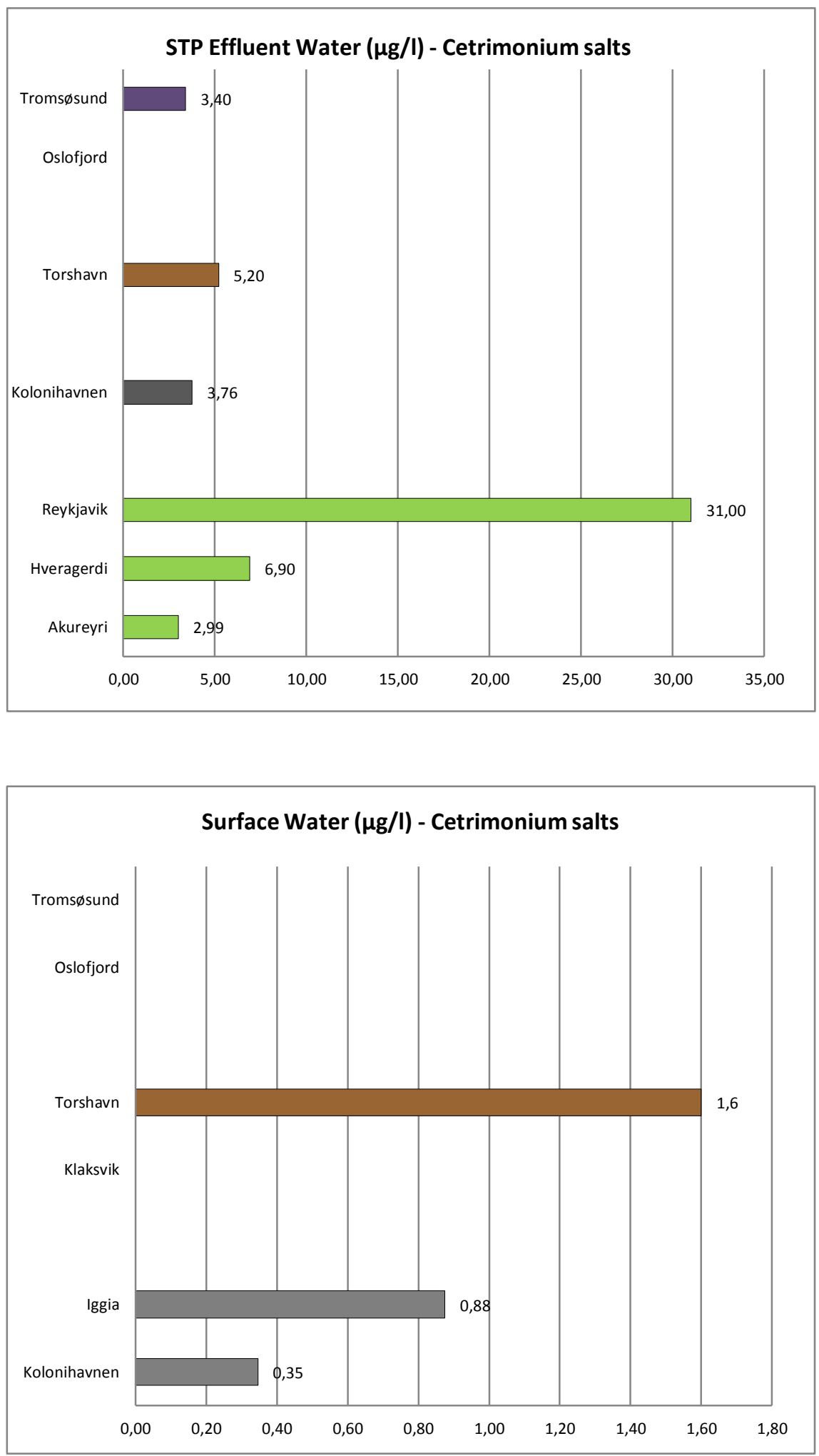


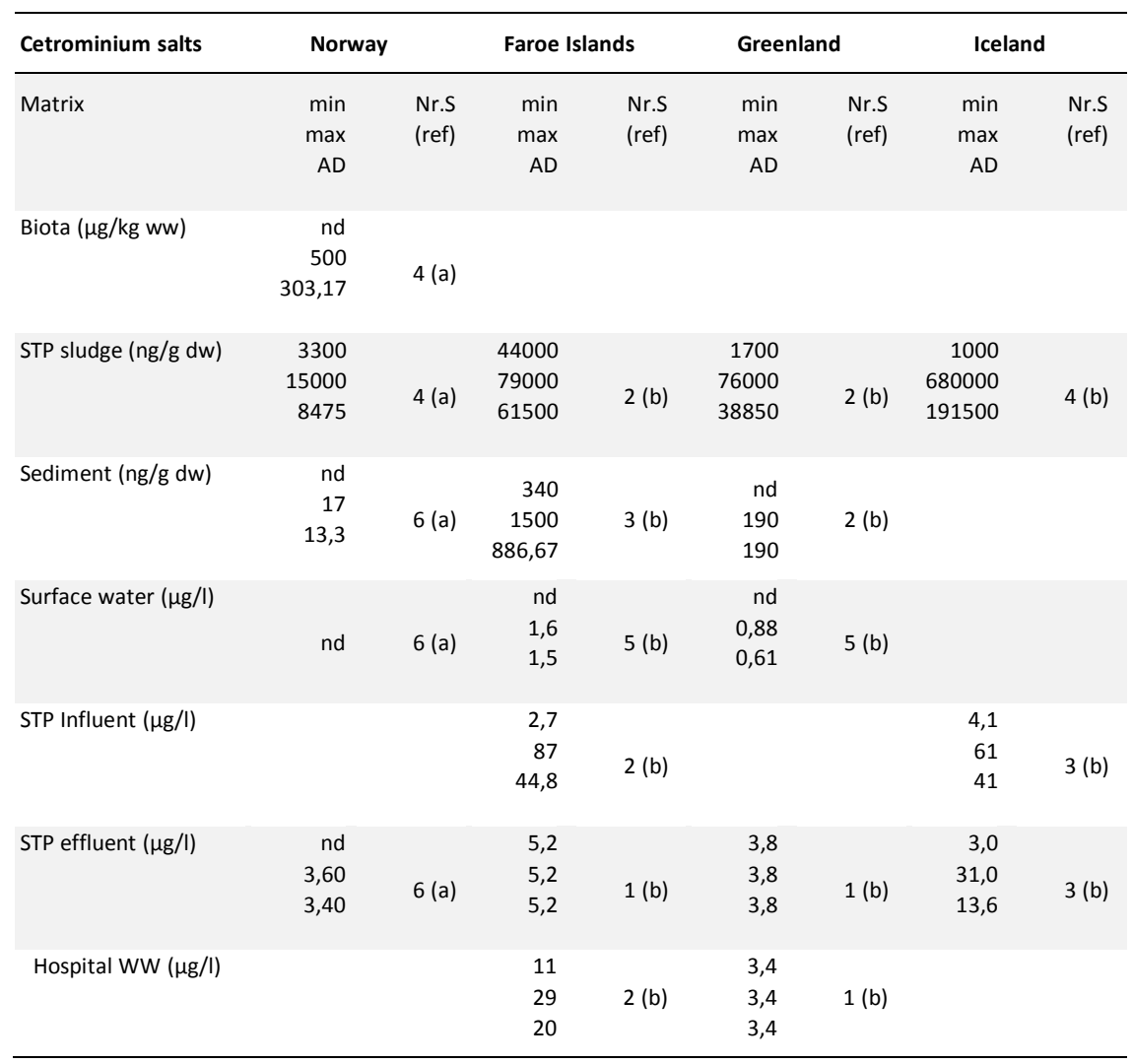

\section{References:}

a) (Schlabach, et al., 2009)

b) (Huber, et al., NA)

\section{Conclusion:}

The compound was monitored in Norway, Faroe Islands, Greenland and Iceland. It was detected in several matrices:

- Biota in Norway

- STP sludge in all countries and in all samples analysed. The values detected were high

- Surface water in Faroe Islands and Greenland, not in Norway. Higher values were detected in Iceland

- STP effluent water in all countries

- Hospital waste water in all countries monitored. Higher values were detected in Faroe Islands

- STP influent in all countries monitored 


\subsection{Compounds monitored in at least two studies}

\subsubsection{Amoxicillin}

CAS nr.: 26787-78-0

Info: Beta-lactam antibiotic

Toxicity data: LOEC (Pseudokirchneriella subcapitata) $=2200 \mathrm{ng} / \mathrm{L}$

PNECaqua $=0,0037 \mu \mathrm{g} / \mathrm{l}$ (Schlabach, et al., 2007)

\begin{tabular}{lcccc}
\hline Amoxicillin & Norway (a) & & Sweden (b) \\
\hline Matrix & Min & Nr.S & Min & Nr.S \\
& $\max$ & (ref) & $\max$ & (ref) \\
Biota & AD & & AD & \\
Sediment & nd & 4 & nd & 9 \\
STP sludge & nd & 6 & nd & 1 \\
STP effluent & nd & 4 & nd & 5 \\
Surface water & nd & 14 & nd & 5 \\
Hospital WW & nd & 11 & & \\
Influent & nd & 5 & nd & 2 \\
\end{tabular}

\section{References:}

a) (Schlabach, et al., 2007)

b) (Johansson, et al., 2003)

\section{Conclusion:}

The compound has been monitored in one study in Norway and one in Sweden. It was not detected in any matrix in either study.

\subsubsection{Ampicillin}

CAS nr.: 69-53-4

Info: beta-lactam antibiotic

Toxicity data: EC50 for bacteriae (Vibrio fischeri) (24 hours): $163 \mathrm{mg} / \mathrm{l}$ (Hellström \& Kreuger, 2005)

PNEC $=45,7 \mu \mathrm{g} / \mathrm{l}$ (Schlabach, et al., 2007)

\begin{tabular}{|c|c|c|c|c|}
\hline \multirow{2}{*}{$\begin{array}{l}\text { Ampicillin } \\
\text { Matrix }\end{array}$} & \multicolumn{2}{|c|}{ Denmark (a) } & \multicolumn{2}{|c|}{ Sweden (b) } \\
\hline & Min & Nr.S & Min & Nr.S \\
\hline & $\max$ & (ref) & $\max$ & (ref) \\
\hline & $A D$ & & $A D$ & \\
\hline STP sludge & nd & 10 & nd & 5 \\
\hline Sediment & & & nd & 1 \\
\hline STP effluent & nd & 9 & nd & 5 \\
\hline Biota & & & nd & 9 \\
\hline Pig slurry & nd & 4 & & \\
\hline influent & nd & 9 & nd & 5 \\
\hline Hospital WW & & & nd & 2 \\
\hline Surface water (up stream and down stream of fish farms) & nd & 16 & & \\
\hline Ground water & nd & 3 & & \\
\hline Warning system for pesticide leaching to groundwater & nd & 8 & & \\
\hline Drain water & nd & 1 & & \\
\hline
\end{tabular}




\section{References:}

a) (Mogensen, et al., 2008)

b) (Johansson, et al., 2003)

\section{Conclusion:}

The compound was monitored in one study in Denmark and one in Sweden. It was not detected in any matrix in either country.

\subsubsection{Benzylpenicillin}

CAS nr.: 61-33-6 (free acid) / 69-57-8 (sodium salt)

Info: beta-lactam antibiotic also known as penicillin $\mathrm{G}$

Toxicity data: LOEC (Microcystis aeruginosa) $=6000 \mathrm{ng} / \mathrm{L}$ (Schlabach, et al., 2009)

$P N E C=N A$

\begin{tabular}{|c|c|c|c|c|}
\hline \multirow{2}{*}{$\begin{array}{l}\text { Benzylpenicillin } \\
\text { Matrix }\end{array}$} & \multicolumn{2}{|c|}{ Denmark (a) } & \multicolumn{2}{|c|}{ Norway (b) } \\
\hline & Min & Nr.S & Min & Nr.S \\
\hline & $\max$ & (ref) & $\max$ & (ref) \\
\hline & $A D$ & & $A D$ & \\
\hline STP sludge & nd & 10 & nd & 4 \\
\hline STP effluent & nd & 9 & nd & 14 \\
\hline Biota (mussel) & & & nd & 4 \\
\hline Sediment & & & nd & 6 \\
\hline Surface water & & & nd & 16 \\
\hline Pig slurry & nd & 4 & & \\
\hline influent & nd & 9 & & \\
\hline Surface water (up stream and down stream of fish farms) & nd & 16 & & \\
\hline Ground water & nd & 3 & & \\
\hline Warning system for pesticide leaching to groundwater & nd & 8 & & \\
\hline Drain water & nd & 1 & & \\
\hline
\end{tabular}

\section{References:}

a) (Mogensen, et al., 2008)

b) (Schlabach, et al., 2009)

\section{Conclusion:}

The compound was monitored in one study in Denmark and one in Norway. The compound was not detected in any sample in either country.

\subsubsection{Cefuroxime}

CAS nr.: 55268-75-2

Info: second-generation cephalosporin antibiotic

Toxicity data:

NOEL $72 \mathrm{hr}$ (Selenastrum capricornutum) $=91 \mathrm{mg} / \mathrm{l}$

NOEL $96 \mathrm{hr}$ (Selenastrum capricornutum) $=120 \mathrm{mg} / \mathrm{l}$

EC50 21 days (Daphnia sp.) $>1000 \mathrm{mg} / \mathrm{l}$

(SFT, 2006)

$P N E C=91 \mu \mathrm{g} / \mathrm{l}$ (Thomas, et al., 2007) 


\begin{tabular}{|c|c|c|c|c|}
\hline LCefuroxime & Norwa & & Sweden & \\
\hline Matrix & $\begin{array}{r}\min \\
\max \\
A D\end{array}$ & $\begin{array}{l}\mathrm{Nr} . \mathrm{S} \\
\text { (ref) }\end{array}$ & $\begin{array}{r}\min \\
\max \\
A D\end{array}$ & $\begin{array}{l}\mathrm{Nr} . \mathrm{S} \\
\text { (ref) }\end{array}$ \\
\hline STP sludge & nd & 7 & nd & 4 \\
\hline STP effluent $(\mu \mathrm{g} / \mathrm{l})$ & nd & 7 & $\begin{array}{r}\text { nd } \\
0,15 \\
0,15\end{array}$ & 5 \\
\hline Biota & & & nd & 9 \\
\hline Sediment & & & nd & 1 \\
\hline Influent ( $\mu \mathrm{g} / \mathrm{l})$ & & & $\begin{array}{l}0,138 \\
0,243 \\
0,195\end{array}$ & 4 \\
\hline Hospital WW & & & nd & 2 \\
\hline
\end{tabular}

\section{References:}

a) (Thomas, et al., 2007)

b) (Johansson, et al., 2003)

\section{Conclusion:}

The compound was monitored in one study in Norway and one in Sweden. It was detected in influent and in only one effluent sample in Sweden, at a concentration below the PNEC. It was also detected in STP influent in Sweden at concentrations below the PNEC.

\subsubsection{Chlortetracycline}

CAS nr.: 57-62-5

Info: Tetracycline antibiotic

Toxicity data:

(Park \& Choi, 2008)

LOEC 7 days (L. gibba) $=0,069-0,65 \mathrm{mg} / \mathrm{l}$

EC50 7 days (M. aeruginosa) $=0,05 \mathrm{mg} / \mathrm{l}$

$P N E C=0,05 \mu \mathrm{g} / \mathrm{l}$ (Park \& Choi, 2008)

\begin{tabular}{|c|c|c|c|c|c|c|}
\hline $\begin{array}{l}\text { Chlortetracycline } \\
\text { Matrix }\end{array}$ & \multicolumn{2}{|c|}{ Denmark (a) } & \multicolumn{2}{|c|}{ Norway (b) } & \multicolumn{2}{|c|}{ Sweden (c) } \\
\hline & $\begin{array}{r}\min \\
\max \\
A D\end{array}$ & $\begin{array}{l}\text { Nr.S } \\
\text { (ref) }\end{array}$ & $\begin{array}{r}\min \\
\max \\
A D\end{array}$ & $\begin{array}{l}\mathrm{Nr} . \mathrm{S} \\
\text { (ref) }\end{array}$ & $\begin{array}{r}\min \\
\max \\
A D\end{array}$ & $\begin{array}{l}\mathrm{Nr} . \mathrm{S} \\
\text { (ref) }\end{array}$ \\
\hline Surface water & nd & $16^{35}$ & & & nd & 18 \\
\hline Groundwater & nd & $3^{36}$ & & & nd & $2^{37}$ \\
\hline
\end{tabular}

35 up and downstream of fish farms

36 include 2 samples in groundwater located by a hospital 


\begin{tabular}{|c|c|c|c|c|c|c|}
\hline Chlortetracycline & Denmark (a) & & Norway (b) & & Sweden (c) & \\
\hline STP Influent & nd & 9 & & & & \\
\hline Effluent & nd & 9 & nd & 7 & & \\
\hline Sediment & & & & & nd & 19 \\
\hline $\begin{array}{l}\text { Warning system for pesticide leaching to ground- } \\
\text { water }(\mathrm{mg} / \mathrm{kg} \mathrm{dw})\end{array}$ & $\begin{array}{l}\text { nd } \\
2,1 \\
2,1\end{array}$ & 9 & & & & \\
\hline Drain water & nd & 1 & & & & \\
\hline Pig slurry & nd & 4 & & & & \\
\hline Manure from farms & & & & & nd & 6 \\
\hline Soil from farms & & & & & nd & 6 \\
\hline STP Influent & nd & 9 & & & & \\
\hline STP sludge & & & nd & 7 & & \\
\hline
\end{tabular}

\section{References:}

a) (Mogensen, et al., 2008)

b) (Thomas, et al., 2007)

c) (Sternbeck, et al., 2007a)

\section{Conclusion:}

The compound was monitored in Denmark, Norway and Sweden and was only detected in one sample for Warning system for pesticide leaching to groundwater. The compound was not detected in any other matrix monitored in either country.

\subsubsection{Demeclocycline}

CAS nr.: 127-33-3

Info: Tetracycline anibiotic Toxicity data:

Toxicity of tetracycline (SFT, 2006)

EC50 7 days (Microcystis aeruginosa) $=0,09 \mathrm{mg} / \mathrm{l}$

EC50 21 days (Daphnia sp.) $=44,8 \mathrm{mg} / \mathrm{l}$

LC50 96 hr (Salvelinus namaycush)= $220 \mathrm{mg} / \mathrm{l}$

PNEC (tetracycline) $=0,09 \mu \mathrm{g} / \mathrm{l}(\mathrm{SFT}, 2006)$ 


\begin{tabular}{|c|c|c|c|c|}
\hline \multirow{2}{*}{$\begin{array}{l}\text { Demeclocycline } \\
\text { Matrix }\end{array}$} & \multicolumn{2}{|c|}{ Norway (a) } & \multicolumn{2}{|c|}{ Sweden (b) } \\
\hline & $\min$ & Nr.S & $\min$ & Nr.S \\
\hline & $\max$ & (ref) & $\max$ & (ref) \\
\hline & $A D$ & & $A D$ & \\
\hline \multirow[t]{3}{*}{ STP sludge $(\mu \mathrm{g} / \mathrm{kg} \mathrm{dw})$} & \multirow{3}{*}{\multicolumn{2}{|c|}{ nd }} & nd & 61 \\
\hline & & & 1200 & \\
\hline & & & 650 & \\
\hline Effluent & \multicolumn{2}{|l|}{ nd } & nd & 52 \\
\hline Biota & & & nd & $3^{38}$ \\
\hline \multirow[t]{3}{*}{ Sediment $(\mu \mathrm{g} / \mathrm{kg} \mathrm{dw})$} & & & nd & \multirow[t]{3}{*}{$7^{39}$} \\
\hline & & & 29 & \\
\hline & & & 29 & \\
\hline Surface water & & & nd & $13^{40}$ \\
\hline Leachates from landfill & & & nd & $10^{41}$ \\
\hline \multirow[t]{3}{*}{ Influent $(\mu \mathrm{g} / \mathrm{l})$} & & & nd & \multirow[t]{3}{*}{19} \\
\hline & & & 0,049 & \\
\hline & & & 0,049 & \\
\hline Manure & & & nd & 5 \\
\hline Hospital WW & & & nd & 7 \\
\hline
\end{tabular}

\section{References:}

a) (Thomas, et al., 2007)

b) (Andersson, et al., 2006)

\section{Conclusion:}

The compound was monitored in one study in Norway and one in Sweden. The compound was detected not detected in any matrix in Norway, but was detected in several matrices in Sweden. It was detected in Sweden in STP sludge, sediment and in only one STP influent sample. The value detected in STP influent was below the PNEC determined for tetracyclines.

\subsubsection{Enrofloxacin}

CAS nr.: 93106-60-6

Info: Fluoroquinolone antibiotic

Toxicity data

EC50 $24 \mathrm{hr}$ (Daphnia magna) $=11,47 \mathrm{mg} / \mathrm{l}$

EC50 5 days (M. aeruginosa) $=0,049 \mathrm{mg} / \mathrm{l}$

LC50 $96 \mathrm{hr}$ (L. macrochirus) > $10 \mathrm{mg} / \mathrm{l}$

\footnotetext{
38 fish for human consumption

39 includes 3 samples from farms

40 includes 7 samples at proximity of farms

41 included 1 treated sample
} 
(Park \& Choi, 2008)

$P N E C=0,049 \mu \mathrm{g} / \mathrm{l}($ Park \& Choi, 2008)

\begin{tabular}{|c|c|c|c|c|c|c|}
\hline $\begin{array}{l}\text { Enrofloxacin } \\
\text { Matrix }\end{array}$ & \multicolumn{2}{|c|}{ Denmark (a) } & \multicolumn{2}{|c|}{ Norway (b) } & \multicolumn{2}{|c|}{ Sweden (c) } \\
\hline & $\begin{array}{r}\min \\
\max \\
A D\end{array}$ & $\begin{array}{l}N r . S \\
\text { (ref) }\end{array}$ & $\begin{array}{r}\min \\
\max \\
A D\end{array}$ & $\begin{array}{l}\text { Nr.S } \\
\text { (ref) }\end{array}$ & $\begin{array}{r}\min \\
\max \\
A D\end{array}$ & $\begin{array}{l}\text { Nr.S } \\
\text { (ref) }\end{array}$ \\
\hline Biota & & & nd & 6 & & \\
\hline Sediment $(\mu \mathrm{g} / \mathrm{kg} \mathrm{dw})$ & & & $\begin{array}{r}\text { nd } \\
17 \\
12,7\end{array}$ & 16 & d & 19 \\
\hline Surface water & nd & $16^{42}$ & nd & 8 & nd & 30 \\
\hline Groundwater & nd & $3^{43}$ & & & nd & $2^{44}$ \\
\hline STP Influent & nd & 9 & nd & 8 & & \\
\hline STP effluent $(\mu \mathrm{g} / \mathrm{l})$ & nd & 9 & nd & 4 & & \\
\hline STP sludge $(\mu \mathrm{g} / \mathrm{kg} \mathrm{dw})$ & nd & 10 & nd & 6 & & \\
\hline Warning system for pesticide leaching to groundwater & nd & 8 & & & & \\
\hline Farm soil & & & & & nd & 6 \\
\hline Drain water & nd & 1 & & & & \\
\hline manure & & & & & nd & 6 \\
\hline Landfill water leachates & & & nd & $9^{45}$ & & \\
\hline Pig slurry & nd & 4 & & & & \\
\hline
\end{tabular}

References:

a) (Mogensen, et al., 2008)

b) (Møskeland, et al., 2006)

c) (Sternbeck, et al., 2007a)

\section{Conclusion:}

The compound was monitored in Denmark, Norway and Sweden. It was only detected in sediment samples from Norway. The compound was not detected in any other matrice in either country.

\footnotetext{
42 upstream and downstream of fish farms

43 including 2 measurements in groundwater at proximity of a hospital

${ }^{44}$ groundwater located at proximity of farms

45 both treated and untreated leachates
} 


\subsubsection{Flumequine}

CAS nr.: 42835-25-6

Info: Synthetic chemotherapeutic antibiotic of the fluoroquinolone drug class

Toxicity data:

EC50 (Microcystis aeruginosa) $=1,96 \mathrm{mg} / \mathrm{l}$

EC50 (Pseudokirchneriella subcapitata) $=5 \mathrm{mg} / \mathrm{l}$

EC50 (Vibrio fischeri) $=0,198 \mathrm{mg} / \mathrm{l}$

(Sternbeck, et al., 2007a)

$P N E C=N A$

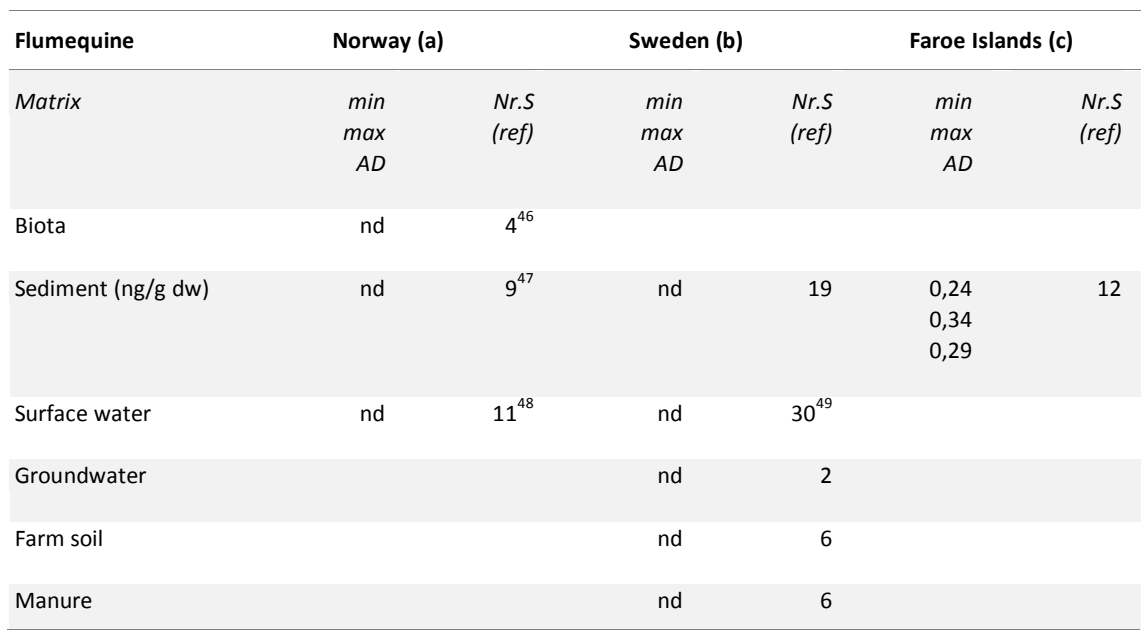

\section{References:}

a) (Schlabach, et al., 2009)

b) (Sternbeck, et al., 2007a)

c) (Gustavson, et al., 2009)

\section{Conclusion:}

The compound was monitored in one Norwegian, one Swedish, and one Faroes Island study. It was detected only in sediment in Faroe Islands.

\subsubsection{Oxolinic Acid}

CAS nr.: 14698-29-4

Info: Quinolone antibiotic

Toxicity data: $N A$

46 mussel

${ }^{47}$ at proximity of fish farms

48 at proximity of fish farms

49 includes sampling at proximity of farms 
PNEC $=0,42 \mu \mathrm{g} / \mathrm{l}$ (Schlabach, et al., 2007)

\begin{tabular}{|c|c|c|c|c|c|c|}
\hline \multirow{2}{*}{$\begin{array}{l}\text { Oxolinic Acid } \\
\text { Matrix }\end{array}$} & \multicolumn{2}{|c|}{ Norway (a) } & \multicolumn{2}{|c|}{ Sweden (b) } & \multicolumn{2}{|c|}{ Faroe Islands (c) } \\
\hline & $\min$ & Nr.S & $\min$ & Nr.S & $\min$ & Nr.S \\
\hline & $\max$ & (ref) & $\max$ & (ref) & $\max$ & (ref) \\
\hline & $A D$ & & $A D$ & & $A D$ & \\
\hline Biota & nd & $4^{50}$ & & & & \\
\hline \multirow[t]{3}{*}{ Sediment $(\mu \mathrm{g} / \mathrm{kg} \mathrm{dw})$} & nd & $9^{51}$ & nd & 19 & 0,45 & 12 \\
\hline & 11,2 & & & & 3,47 & \\
\hline & 5,57 & & & & 1,91 & \\
\hline \multirow[t]{3}{*}{ Surface water $(\mu \mathrm{g} / \mathrm{l})$} & nd & $10^{52}$ & nd & $30^{53}$ & & \\
\hline & 0,002 & & & & & \\
\hline & 0,0015 & & & & & \\
\hline Groundwater & & & nd & 2 & & \\
\hline Farm soil & & & nd & 6 & & \\
\hline Manure & & & nd & 6 & & \\
\hline
\end{tabular}

\section{References:}

a) (Schlabach, et al., 2009)

b) (Sternbeck, et al., 2007a)

c) (Gustavson, et al., 2009)

\section{Conclusion:}

The compound was monitored in one Swedish, one Norwegian study and one study in Faroe Islands

It was not detected in any samples from Sweden

The compound was detected in sediment samples and surface water samples at proximity of fish farms in Norway

The compound was detected in sediment samples samples at proximity of fish farms in Faroe Islands

\subsubsection{Penicillin $V$}

CAS nr.: 87-08-1

Info: Penicillin antibiotic

Toxicity data: NA

$P N E C=\mathrm{NA}$

50 mussel

51 at proximity of fish farms

52 at proximity of fish farms

53 includes sampling at proximity of farms 


\begin{tabular}{lcccc}
\hline Penicillin V & Denmark (a) & & \multicolumn{2}{c}{ Sweden (b) } \\
\hline Matrix & Mi & Nr.S & min & Nr.S \\
& $\max$ & (ref) & max & (ref) \\
Biota & $A D$ & & $A D$ & nd \\
Effluent & & & nd & 5 \\
Sediment & nd & 6 & nd & 1 \\
STP sludge & & & nd & 5 \\
STP Influent & & & nd & 5 \\
Hospital WW & & & nd & 2 \\
\hline
\end{tabular}

\section{References:}

a) (Kjølholt, et al., 2003)

b) (Johansson, et al., 2003)

\section{Conclusion:}

The compound was monitored in one Danish and one Swedish study, but was not detected in any matrice sampled in either country.

\subsubsection{Silver}

CAS nr.: 7440-22-4

Info: Antiseptic and desinfectant

Toxicity data

$\mathrm{Ag}^{+}$LC50 7day (Oncorhynchus mykiss) $=0.0032 \mathrm{mg} / \mathrm{l}$

(Svenson, et al., 2009)

$P N E C=\mathrm{NA}$

\begin{tabular}{|c|c|c|c|c|}
\hline \multirow{2}{*}{$\begin{array}{l}\text { Silver } \\
\text { Matrix }\end{array}$} & \multicolumn{2}{|c|}{ Norway } & \multicolumn{2}{|c|}{ Sweden } \\
\hline & $\min$ & Nr.S & $\min$ & Nr.S \\
\hline & $\max$ & (ref) & $\max$ & (ref) \\
\hline & $A D$ & & $A D$ & \\
\hline \multirow[t]{3}{*}{$\operatorname{Biota}(\mathrm{mg} / \mathrm{kg} \mathrm{dw})$} & 0,003 & 9 (a) & & \\
\hline & 0,019 & & & \\
\hline & 0,085 & & & \\
\hline \multirow[t]{3}{*}{$\operatorname{Biota}(\mu \mathrm{g} / \mathrm{kg}$ fat weight) } & & & nd & 21 (c) \\
\hline & & & 1,0 & \\
\hline & & & 0,65 & \\
\hline \multirow[t]{3}{*}{ Sediment (mg/kg dw) } & 192,6 & 7 (b) & 0,1 & 49 (c) \\
\hline & 426,6 & & 44 & \\
\hline & 310,8 & & 3,03 & \\
\hline \multirow[t]{3}{*}{ Sludge (mg/kg dw) } & & & 0,12 & 45 (c) \\
\hline & & & 88,7 & \\
\hline & & & 10,78 & \\
\hline \multirow[t]{3}{*}{ Surface water $(\mu \mathrm{g} / \mathrm{l})$} & & & nd & $27(c d)$ \\
\hline & & & 0,06 & \\
\hline & & & 0,015 & \\
\hline \multirow[t]{3}{*}{ Influent ( $\mu \mathrm{g} / \mathrm{l})$} & & & nd & $137(\mathrm{~cd})$ \\
\hline & & & 1,4 & \\
\hline & & & 0,05 & \\
\hline \multirow[t]{3}{*}{ Hospital waste water $(\mu \mathrm{g} / \mathrm{l})$} & & & 0,006 & 8 (c) \\
\hline & & & 0,07 & \\
\hline & & & 0,022 & \\
\hline
\end{tabular}




\begin{tabular}{|c|c|c|c|}
\hline Silver & Norway & Swed & \\
\hline \multirow[t]{3}{*}{ Laundry effluent $(\mu \mathrm{g} / \mathrm{l})$} & & 0,054 & 1 (c) \\
\hline & & 0,054 & \\
\hline & & 0,054 & \\
\hline \multirow[t]{3}{*}{ Landfill runoff ( $\mu \mathrm{g} / \mathrm{l})$} & & 0,013 & 9 (c) \\
\hline & & 0,83 & \\
\hline & & 0,29 & \\
\hline \multirow[t]{3}{*}{ Urban runoff $(\mu \mathrm{g} / \mathrm{l})$} & & 0,018 & 7 (c) \\
\hline & & 1,9 & \\
\hline & & 0,39 & \\
\hline \multirow[t]{3}{*}{ Drinking water $(\mu \mathrm{g} / \mathrm{l})$} & & nd & 5 (c) \\
\hline & & 0,011 & \\
\hline & & 0,011 & \\
\hline \multirow[t]{3}{*}{ Power plant condensate $(\mu \mathrm{g} / \mathrm{l})$} & & 0,049 & 1 (c) \\
\hline & & 0,049 & \\
\hline & & 0,049 & \\
\hline
\end{tabular}

\section{References:}

a) (Green, et al., 2009)

b) (Evenset, et al., 2009)

c) (Svenson, et al., 2009)

d) (Lilja, et al., 2010)

\section{Conclusion:}

The compound was monitored in two Swedish and two Norwegian studies and was detected in all matrices monitored in both countries, including surface water and in one drinking water sample. The compound was detected in higher concentraions in biota in Sweden than in Norway. The concentrations detected in sediment were high and ca. 100 times higher in Norway than in Sweden.

\subsubsection{Spiramycin}

CAS nr.: 8025-81-8

Info: Macrolide antibiotic

Toxicity data: LOEC (Microcystis aeruginosa) $=7 \mu \mathrm{g} / \mathrm{l}$ (Schlabach, et al., 2009)

PNEC $=0,005 \mu \mathrm{g} / \mathrm{l}$ (Schlabach, et al., 2007)

\begin{tabular}{lcccc}
\hline Spiramycin & Norway (a) & \multicolumn{3}{c}{ Sweden (b) } \\
\hline Matrix & $\min$ & Nr.S & $\min$ & Nr.S \\
& $\max$ & $($ ref) & $\max$ & (ref) \\
& $A D$ & & $A D$ & \\
Biota & nd & $3^{54}$ & &
\end{tabular}

${ }^{54}$ mussel 


\begin{tabular}{lcccr}
\hline Spiramycin & Norway (a) & \multicolumn{2}{c}{ Sweden (b) } \\
\hline Sediment & nd & $6^{55}$ & nd & 19 \\
STP sludge & nd & 4 & & \\
STP effluent $(\mu \mathrm{g} / \mathrm{l})$ & nd & 10 & & $30^{57}$ \\
& 0,03 & & nd & 2 \\
Surface water & 0,02 & $12^{56}$ & nd & 6 \\
Groundwater & nd & & nd & 6 \\
Farm soil & & & nd & 6 \\
Manure & & & & 6
\end{tabular}

\section{References:}

a) (Schlabach, et al., 2009)

b) (Sternbeck, et al., 2007a)

\section{Conclusion:}

The compound was monitored in one Swedish and one Norwegian study and was only detected in STP effluent in Norway. The compound was not detected in any other matrice sampled in either country.

\subsubsection{Sulfadimidine}

CAS nr.: 57-68-1

Info: A sulfonamide antibacterial

Toxicity data: NA

$P N E C=\mathrm{NA}$

\begin{tabular}{lcccc}
\hline Sulfadimidine & Denmark (a) & & Sweden (b) \\
\hline Matrix & min & Nr.S & $\min$ & Nr.S \\
& $\max$ & $($ ref) & $\max$ & (ref) \\
Sediment & $A D$ & & $A D$ & \\
STP sludge & & & nd & 19 \\
Effluent & nd & 10 & & \\
Surface water & nd & 9 & & \\
Pig slurry & nd & $16^{58}$ & nd & $30^{59}$ \\
Groundwater & nd & 3 & & \\
Warning system for pesticide leaching to groundwater & nd & $3^{60}$ & & \\
Drain water & nd & 9 & & \\
Influent & nd & 1 & & \\
Ground water & nd & 9 & nd
\end{tabular}

\footnotetext{
55 at proximity of fish farms

56 at proximity of fish farms

57 includes sampling at proximity of farms

${ }^{58}$ up and downstream of fish farms

59 includes sampling at proximity of farms

60 including 2 at proximity of hospital
} 


\begin{tabular}{lccc}
\hline Sulfadimidine & Denmark (a) & Sweden (b) \\
\hline Farm soil & nd & 6 \\
manure & nd & 6 \\
\hline
\end{tabular}

\section{References:}

a) (Mogensen, et al., 2008)

b) (Sternbeck, et al., 2007a)

\section{Conclusion:}

The compound was monitored in one Swedish and one Danish study and was not detected in any matrix in either country.

\subsubsection{Sulfadoxine}

CAS nr.: 2447-57-6

Info: An ultra-long-lasting sulfonamide

Toxicity data: NA

$P N E C=\mathrm{NA}$

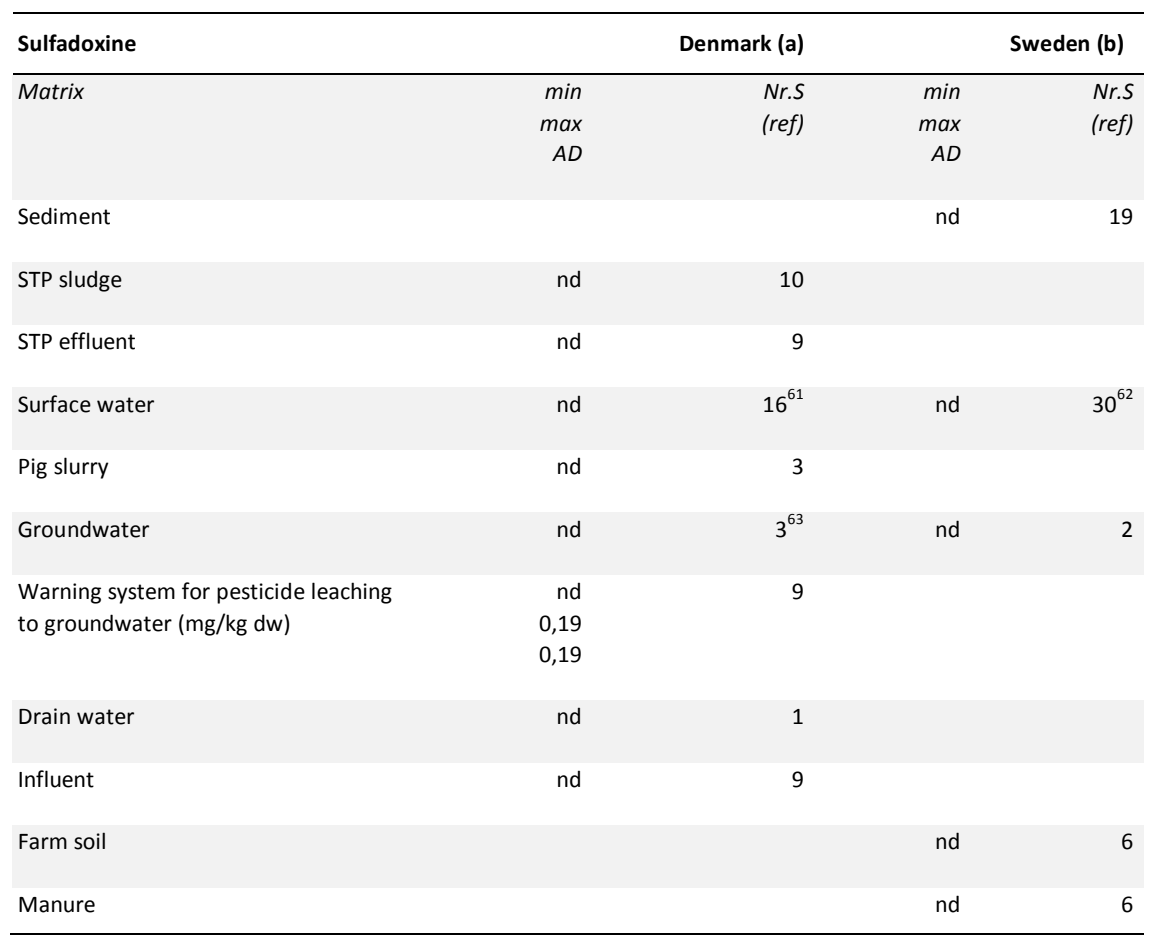

61 up and downstream of fish farms

62 includes sampling at proximity of farms

63 including 2 at proximity of hospital 


\section{References:}

a) (Mogensen, et al., 2008)

b) (Sternbeck, et al., 2007a)

\section{Conclusion:}

The compound was monitored in one Swedish and one Danish study and was detected in only one sample for Warning system for pesticide leaching to groundwater.The compound was not detected in any other matrix in either country.

\subsubsection{Tylosine}

CAS nr.: 1401-69-0

Info: Macrolide-class antibiotic used in veterinary medicine Toxicity data:

EC50 7 days (Microcystis aeruginosa) $=34 \mu \mathrm{g} / \mathrm{l}$

NOEC 7 days (Lemna gibba) $=300-1000 \mu \mathrm{g} / \mathrm{l}$

NOEC 21 days (Daphnia magna) $=45000 \mu \mathrm{g} / \mathrm{l}$

EC50 $72 \mathrm{hr}$ (Selenastrum capricornutum) $=950 \mu \mathrm{g} / \mathrm{l}$

EC50 21 days (Aporrectodea caliginosa) $=4530 \mathrm{mg} / \mathrm{kg} \mathrm{dw}$

(Sternbeck, et al., 2007a)

$P N E C=\mathrm{NA}$

\begin{tabular}{|c|c|c|c|c|}
\hline \multirow[b]{2}{*}{ Matrix } & \multicolumn{2}{|c|}{ Denmark (a) } & \multicolumn{2}{|c|}{ Sweden (b) } \\
\hline & $\min$ & Nr.S & $\min$ & Nr.S \\
\hline & $\max$ & (ref) & $\max$ & (ref) \\
\hline & $A D$ & & $A D$ & \\
\hline Sediment & & & nd & 19 \\
\hline STP sludge & nd & 10 & & \\
\hline Effluent & nd & 9 & & \\
\hline Surface water & nd & $16^{64}$ & nd & 30 \\
\hline Pig slurry & nd & 3 & & \\
\hline Groundwater & nd & $3^{65}$ & nd & 2 \\
\hline Warning system for pesticide leaching to groundwater $(\mathrm{mg} / \mathrm{kg} \mathrm{dw})$ & nd & 9 & & \\
\hline Drain water & nd & 1 & & \\
\hline Influent & nd & 9 & & \\
\hline Farm soil & & & nd & 6 \\
\hline Manure & & & nd & 6 \\
\hline
\end{tabular}

\section{References:}

a) (Mogensen, et al., 2008)

b) (Sternbeck, et al., 2007a)

${ }^{64}$ up and downstream of fish farms

65 including 2 at proximity of hospital 


\section{Conclusion:}

The compound was monitored in one Swedish and one Danish study and was not detected in any matrix in either country.

\subsection{Compounds monitored in one study}

\begin{tabular}{|c|c|c|c|c|c|c|}
\hline & $\begin{array}{r}1,2,3,4- \\
\text { Tetrachloro } \\
\text { benzene } \\
(634-66-2)\end{array}$ & $\begin{array}{r}1,3,5- \\
\text { Trichloro- } \\
\text { benzene } \\
(108-70-3)\end{array}$ & $\begin{array}{r}1,2,4- \\
\text { Trichloro- } \\
\text { benzene } \\
(120-82-1)\end{array}$ & $\begin{array}{r}1,2,3- \\
\text { Trichloro- } \\
\text { benzene } \\
(87-61-6)\end{array}$ & $\begin{array}{r}1,2,3,5-+ \\
1,2,4,5- \\
\text { Tetrachloro- } \\
\text { benzene }\end{array}$ & \\
\hline \multirow[t]{3}{*}{ Matrix } & $\min$ & $\min$ & $\min$ & $\min$ & $\min$ & Nr.S \\
\hline & $\max$ & $\max$ & $\max$ & $\max$ & $\max$ & \\
\hline & $A D$ & $A D$ & $A D$ & $A D$ & $A D$ & \\
\hline $\begin{array}{l}\text { STP } \\
\text { influent }\end{array}$ & nd & nd & nd & nd & nd & 7 \\
\hline
\end{tabular}

\section{Reference:}

- (Lilja, et al., 2010) 


\begin{tabular}{|c|c|c|c|c|c|c|c|c|c|c|c|c|c|c|}
\hline \multirow[b]{2}{*}{ Matrix } & \multicolumn{2}{|c|}{$\begin{array}{l}\text { Bronopol } \\
(52-51-7)\end{array}$} & \multicolumn{2}{|c|}{$\begin{array}{c}\text { DDMAC } \\
(7173-51-5)\end{array}$} & \multicolumn{2}{|c|}{$\begin{array}{l}\text { Propiconazole } \\
(60207-90-1)\end{array}$} & \multicolumn{2}{|c|}{$\begin{array}{l}\text { Resorcinol } \\
(108-46-3)\end{array}$} & \multicolumn{2}{|c|}{$\begin{array}{c}\text { 2(Tiocyano- } \\
\text { methylthio)benzo- } \\
\text { thiazole (21564-17-0) }\end{array}$} & \multicolumn{2}{|c|}{$\begin{array}{c}\text { 4-Chloro-3-cresol } \\
\text { (59-50-7) }\end{array}$} & \multicolumn{2}{|c|}{$\begin{array}{l}\text { 4-chloro-3- } \\
\text { methylphenol } \\
\text { (59-50-7) }\end{array}$} \\
\hline & $\begin{array}{r}\min \\
\max \\
A D\end{array}$ & Nr.S & $\begin{array}{r}\min \\
\max \\
A D\end{array}$ & Nr.S & $\begin{array}{r}\min \\
\max \\
A D\end{array}$ & Nr.S & $\begin{array}{r}\min \\
\max \\
A D\end{array}$ & Nr.S & $\begin{array}{r}\min \\
\max \\
A D\end{array}$ & Nr.S & $\begin{array}{r}\min \\
\max \\
A D\end{array}$ & Nr.S & $\begin{array}{r}\min \\
\max \\
A D\end{array}$ & Nr.S \\
\hline Air (ng/m3) & nd & 9 & & & $\begin{array}{r}\text { nd } \\
0,39 \\
0,3\end{array}$ & $6^{66}$ & $\begin{array}{l}\text { nd } \\
\text { nd } \\
\text { nd }\end{array}$ & 9 & & & & & nd & 6 \\
\hline Biota & nd & 8 & nd & 4 & $\begin{array}{l}\text { nd } \\
\text { nd } \\
\text { nd }\end{array}$ & 7 & $\begin{array}{l}\text { nd } \\
\text { nd } \\
\text { nd }\end{array}$ & 8 & nd & 1 & nd & 1 & nd & 6 \\
\hline $\begin{array}{l}\text { Sediment } \\
(\mu \mathrm{g} / \mathrm{kg} \mathrm{dw})\end{array}$ & nd & 15 & nd & 15 & $\begin{array}{l}\text { nd } \\
\text { nd } \\
\text { nd }\end{array}$ & 17 & $\begin{array}{l}\text { nd } \\
\text { nd } \\
\text { nd }\end{array}$ & 17 & nd & 7 & nd & 7 & nd & 9 \\
\hline $\begin{array}{l}\text { STP sludge } \\
(\mu g / k g d w)\end{array}$ & nd & 14 & $\begin{array}{r}\text { nd } \\
61000 \\
13080\end{array}$ & 25 & $\begin{array}{l}\text { nd } \\
54 \\
36\end{array}$ & 28 & $\begin{array}{r}\text { nd } \\
380 \\
187\end{array}$ & 22 & nd & 11 & $\begin{array}{r}\text { nd } \\
40 \\
19,6\end{array}$ & 11 & $\begin{array}{r}\text { nd } \\
67 \\
25,1\end{array}$ & 19 \\
\hline $\begin{array}{l}\text { STP effluent } \\
(\mu \mathrm{g} / \mathrm{l})\end{array}$ & nd & 2 & nd & 2 & $\begin{array}{r}\text { nd } \\
0,1 \\
0,06\end{array}$ & 7 & $\begin{array}{l}\text { nd } \\
\text { nd } \\
\text { nd }\end{array}$ & 2 & & & & & $\begin{array}{r}\text { nd } \\
0,02 \\
0,02\end{array}$ & 4 \\
\hline $\begin{array}{l}\text { Influent water } \\
(\mu \mathrm{g} / \mathrm{l})\end{array}$ & nd & 1 & nd & 1 & $\begin{array}{l}\text { nd } \\
\text { nd } \\
\text { nd }\end{array}$ & 1 & $\begin{array}{l}0,078 \\
0,078 \\
0,078\end{array}$ & 1 & nd & 1 & nd & 1 & & \\
\hline $\begin{array}{l}\text { Surface water } \\
(\mu \mathrm{g} / \mathrm{l})\end{array}$ & nd & 19 & nd & 22 & $\begin{array}{r}\text { nd } \\
0,03\end{array}$ & 24 & $\begin{array}{l}\text { nd } \\
\text { nd }\end{array}$ & 25 & nd & 16 & $\begin{array}{r}\text { nd } \\
0,11\end{array}$ & 16 & nd & 9 \\
\hline
\end{tabular}

${ }^{66}$ was only detected in air samples from a wood impregnation plant 


\begin{tabular}{|c|c|c|c|c|c|c|c|c|c|c|c|}
\hline & $\begin{array}{l}\text { Bronopol } \\
(52-51-7)\end{array}$ & & $\begin{array}{c}\text { DDMAC } \\
\text { (7173-51-5) }\end{array}$ & & $\begin{array}{l}\text { Propiconazole } \\
\text { (60207-90-1) }\end{array}$ & & $\begin{array}{l}\text { Resorcinol } \\
(108-46-3)\end{array}$ & $\begin{array}{c}\text { 2(Tiocyano- } \\
\text { methylthio)benzo- } \\
\text { thiazole (21564-17-0) }\end{array}$ & $\begin{array}{c}\text { 4-Chloro-3-cresol } \\
(59-50-7)\end{array}$ & $\begin{array}{c}\text { 4-chloro-3- } \\
\text { methylphenol } \\
(59-50-7)\end{array}$ & \\
\hline Precipitation & nd & 3 & & & & & $\begin{array}{l}\text { nd } \\
\text { nd } \\
\text { nd }\end{array}$ & 2 & & & \\
\hline $\begin{array}{l}\text { Deposition } \\
\text { (ng/m2.d) }\end{array}$ & & & & & $\begin{array}{l}\text { nd } \\
\text { nd } \\
\text { nd }\end{array}$ & 3 & & & & $\begin{array}{l}\text { nd } \\
1,8 \\
1,8\end{array}$ & 3 \\
\hline $\begin{array}{l}\text { Leachates } \\
\text { from land-fill } \\
(\mu \mathrm{g} / \mathrm{l})\end{array}$ & & & nd & 3 & $\begin{array}{l}\text { nd } \\
\text { nd } \\
\text { nd }\end{array}$ & 3 & & & & $\begin{array}{l}\text { nd } \\
0,7 \\
0,7\end{array}$ & 3 \\
\hline Food & nd & 5 & nd & 6 & & & & & & & \\
\hline
\end{tabular}

\section{Reference:}

- (Remberger, et al., 2006) 


\begin{tabular}{|c|c|c|c|c|c|c|c|c|c|c|c|c|}
\hline & $\begin{array}{c}\text { Danofloxacin } \\
(112398-08-0)\end{array}$ & $\begin{array}{r}\text { Difloxacin } \\
(3116-76-5)\end{array}$ & $\begin{array}{r}\text { Dihydro- } \\
\text { streptomycin } \\
(128-46-1)\end{array}$ & $\begin{array}{l}\text { Gentamycin } \\
\text { (1403-66-3) }\end{array}$ & $\begin{array}{l}\text { Marbofloxacin } \\
\text { (1115550-35-1) }\end{array}$ & $\begin{array}{l}\text { Sarafloxacin } \\
\text { (98105-99-8) }\end{array}$ & $\begin{array}{r}\text { Sulfabenz- } \\
\text { amide } \\
(127-71-9)\end{array}$ & $\begin{array}{r}\text { Sulfacet- } \\
\text { amide } \\
(144-80-9)\end{array}$ & $\begin{array}{r}\text { Sulfachloro- } \\
\text { pyridazine } \\
(80-32-0)\end{array}$ & $\begin{array}{l}\text { Sulfaclozin } \\
\text { (102-65-8) }\end{array}$ & $\begin{array}{r}\text { Sulfadi- } \\
\text { methoxine } \\
(122-11-2)\end{array}$ & \\
\hline \multirow[t]{3}{*}{ Matrix } & $\min$ & $\min$ & $\min$ & $\min$ & $\min$ & $\min$ & $\min$ & $\min$ & $\min$ & $\min$ & $\min$ & Nr.S \\
\hline & $\max$ & $\max$ & $\max$ & $\max$ & $\max$ & $\max$ & $\max$ & $\max$ & $\max$ & $\max$ & $\max$ & \\
\hline & $A D$ & $A D$ & $A D$ & $A D$ & $A D$ & $A D$ & $A D$ & $A D$ & $A D$ & $A D$ & $A D$ & \\
\hline Sediment $(\mu \mathrm{g} / \mathrm{kg} \mathrm{dw})$ & nd & nd & nd & nd & nd & nd & nd & nd & nd & nd & nd & 19 \\
\hline Surface water (ng/l) & nd & nd & nd & nd & nd & nd & nd & nd & nd & nd & nd & 30 \\
\hline Ground water (ng/l) & nd & nd & nd & nd & nd & nd & nd & nd & nd & nd & nd & 2 \\
\hline Manure $(\mu \mathrm{g} / \mathrm{kg} \mathrm{dw})$ & nd & nd & nd & nd & nd & nd & nd & nd & nd & nd & nd & 6 \\
\hline \multirow[t]{2}{*}{ Soil ( $\mu \mathrm{g} / \mathrm{kg} \mathrm{dw})$} & nd & nd & nd & nd & nd & nd & nd & nd & nd & nd & nd & 6 \\
\hline & $\begin{array}{r}\text { Sulphafurazole } \\
(127-69-5)\end{array}$ & $\begin{array}{r}\text { Sulfaguanidine } \\
(57-67-0)\end{array}$ & $\begin{array}{r}\text { Sulfamerazine } \\
(127-79-7)\end{array}$ & $\begin{array}{l}\text { Sulfameter } \\
\text { (651-06-9) }\end{array}$ & $\begin{array}{r}\text { Sulfamethoxy- } \\
\text { pyridazine } \\
(000080-35-3)\end{array}$ & $\begin{array}{c}\text { Sulfamono- } \\
\text { methoxine } \\
(1220-83-3)\end{array}$ & $\begin{array}{r}\text { Sulfamoxole } \\
(729-99-7)\end{array}$ & $\begin{array}{r}\text { Sulfanilamide } \\
(63-74-1)\end{array}$ & $\begin{array}{r}\text { Sulfapyridine } \\
\text { (000144-83-2) }\end{array}$ & $\begin{array}{r}\text { Sulfaquinox- } \\
\text { aline } \\
(59-40-5)\end{array}$ & $\begin{array}{r}\text { Sulfatiazol } \\
872-14-0)\end{array}$ & \\
\hline \multirow[t]{3}{*}{ Matrix } & $\min$ & $\min$ & $\min$ & $\min$ & $\min$ & $\min$ & $\min$ & $\min$ & $\min$ & $\min$ & $\min$ & Nr.S \\
\hline & $\max$ & $\max$ & $\max$ & $\max$ & $\max$ & $\max$ & $\max$ & $\max$ & $\max$ & $\max$ & $\max$ & \\
\hline & $A D$ & $A D$ & $A D$ & $A D$ & $A D$ & $A D$ & $A D$ & $A D$ & $A D$ & $A D$ & $A D$ & \\
\hline Sediment $(\mu \mathrm{g} / \mathrm{kg} \mathrm{dw})$ & nd & nd & nd & nd & nd & nd & nd & nd & nd & nd & nd & 19 \\
\hline Surface water (ng/l) & nd & nd & nd & nd & nd & nd & nd & nd & nd & nd & nd & 30 \\
\hline Ground water (ng/l) & nd & nd & nd & nd & nd & nd & nd & nd & nd & nd & nd & 2 \\
\hline Manure $(\mu \mathrm{g} / \mathrm{kg} \mathrm{dw})$ & nd & nd & nd & nd & nd & nd & nd & nd & nd & nd & nd & 6 \\
\hline Soil ( $\mu \mathrm{g} / \mathrm{kg} \mathrm{dw})$ & nd & nd & nd & nd & nd & nd & nd & nd & nd & nd & nd & 6 \\
\hline
\end{tabular}

\section{Reference:}

- (Sternbeck, et al., 2007) 


\begin{tabular}{|c|c|c|c|c|c|c|c|}
\hline & $\begin{array}{l}\text { Azithromycin } \\
\text { (83905-01-5) }\end{array}$ & $\begin{array}{r}\text { Clarithromycine } \\
(81103-11-9)\end{array}$ & $\begin{array}{l}\text { Clindamycin } \\
\text { (18323-44-9) }\end{array}$ & $\begin{array}{r}\text { Roxithromycin } \\
(80214-83-1)\end{array}$ & $\begin{array}{r}\text { Fluconazole } \\
(86386-73-4)\end{array}$ & $\begin{array}{r}\text { Miconazol } \\
(2013-58-3)\end{array}$ & \\
\hline \multirow[t]{3}{*}{ Matrix } & $\min$ & $\min$ & $\min$ & $\min$ & $\min$ & $\min$ & Nr.S \\
\hline & $\max$ & $\max$ & $\max$ & $\max$ & $\max$ & $\max$ & \\
\hline & $A D$ & $A D$ & $A D$ & $A D$ & $A D$ & $A D$ & \\
\hline \multirow[t]{3}{*}{ Biota $(\mu \mathrm{g} / \mathrm{kg})$} & nd & nd & nd & nd & nd & nd & 15 \\
\hline & & & & & 0,9 & & \\
\hline & & & & & 0,9 & & \\
\hline \multirow[t]{3}{*}{ Sludge ( $\mu \mathrm{g} / \mathrm{kg} d w)$} & nd & nd & 5,9 & nd & 2,6 & 170 & 5 \\
\hline & 5,6 & 13 & 21 & & 47 & 410 & \\
\hline & 5,6 & 5,23 & 15,2 & & 13,84 & 262 & \\
\hline \multirow[t]{3}{*}{ Effluent (ng/l) } & nd & nd & 31 & nd & 55 & nd & 13 \\
\hline & 27 & 780 & 280 & 980 & 1100 & 9,2 & \\
\hline & 15,3 & 123,6 & 137,8 & 252,8 & 373,6 & 7,7 & \\
\hline \multirow[t]{3}{*}{ Surface water (ng/l) } & nd & nd & nd & nd & 1,8 & nd & 15 \\
\hline & 27 & 1100 & 140 & 1100 & 290 & & \\
\hline & 16,2 & 165,2 & 37,3 & 463,3 & 50,18 & & \\
\hline \multirow[t]{3}{*}{ STP influent (ng/l) } & nd & 38 & 37 & nd & 97 & nd & 12 \\
\hline & 44 & 620 & 230 & 890 & 2100 & 48 & \\
\hline & 13,5 & 262,4 & 97,7 & 398,8 & 679,7 & 14,3 & \\
\hline \multirow[t]{3}{*}{ Drinking water (ng/l) } & nd & nd & nd & nd & nd & nd & 6 \\
\hline & & & & & 3,3 & & \\
\hline & & & & & 2,63 & & \\
\hline
\end{tabular}

Reference:

- (Fick, et al., 2011) 


\begin{tabular}{|c|c|c|c|}
\hline & $\begin{array}{r}\text { Cefadroxil } \\
(66592-87-8)\end{array}$ & $\begin{array}{r}\text { Metronidazol } \\
(443-48-1)\end{array}$ & \\
\hline \multirow[t]{3}{*}{ Matrix } & $\min$ & $\min$ & Nr.S \\
\hline & $\max$ & $\max$ & \\
\hline & $A D$ & $A D$ & \\
\hline Biota & nd & nd & 9 \\
\hline Sediment & nd & nd & 1 \\
\hline STP sludge & nd & nd & 5 \\
\hline \multirow[t]{3}{*}{ STP Influent ( $\mu \mathrm{g} / \mathrm{l})$} & nd & nd & 5 \\
\hline & 0,077 & 0,081 & \\
\hline & 0,077 & 0,081 & \\
\hline \multirow[t]{3}{*}{ STP effluent ( $\mu \mathrm{g} / \mathrm{l})$} & nd & nd & 5 \\
\hline & 0,082 & & \\
\hline & 0,082 & & \\
\hline \multirow[t]{3}{*}{ Hospital WW ( $\mu \mathrm{g} / \mathrm{l})$} & nd & nd & 2 \\
\hline & & 45,15 & \\
\hline & & 45,15 & \\
\hline
\end{tabular}

\section{References:}

- (Johansson, et al., 2003)

\begin{tabular}{|c|c|c|}
\hline & Chlorocycline (57-62-5) & \\
\hline \multirow[t]{3}{*}{ Matrix } & $\min$ & Nr.S \\
\hline & $\max$ & \\
\hline & $A D$ & \\
\hline Biota & nd & 3 \\
\hline Sediment $(\mu \mathrm{g} / \mathrm{kg} \mathrm{dw})$ & nd & 7 \\
\hline STP sludge $(\mu \mathrm{g} / \mathrm{kg} \mathrm{dw})$ & nd & 60 \\
\hline \multirow[t]{3}{*}{ STP effluent $(\mu \mathrm{g} / \mathrm{l})$} & nd & 52 \\
\hline & 0,68 & \\
\hline & 0,26 & \\
\hline \multirow[t]{3}{*}{ Influent water ( $\mu \mathrm{g} / \mathrm{l})$} & nd & 19 \\
\hline & 0,34 & \\
\hline & 0,26 & \\
\hline Surface water & nd & 5 \\
\hline \multirow[t]{3}{*}{ Leachates from landfill $(\mu \mathrm{g} / \mathrm{l})$} & nd & $8 d$ \\
\hline & 0,005 & \\
\hline & 0,005 & \\
\hline \multirow[t]{3}{*}{ Hospital WW ( $\mu \mathrm{g} / \mathrm{l})$} & nd & 12 \\
\hline & 0,001 & \\
\hline & 0,001 & \\
\hline Manure & nd & 5 \\
\hline
\end{tabular}

\section{Reference:}

- (Andersson, et al., 2006) 


\begin{tabular}{lcc}
\hline & Zinc pyrithione (13463-41-7) & Nr.S \\
\hline Matrix & $\min$ & $\max$ \\
& $A D$ & 16 \\
Sediment & nd & 34 \\
STP sludge & nd & 40 \\
STP effluent and influent (ng/l) & nd & \\
& 17 & 13 \\
Surface water (ng/l) & 9,45 & 1 \\
Landfill leachates (ng/l) & nd & nd \\
Hospital WW (ng/l) & nd & 32 \\
\end{tabular}

\section{Reference:}

- (Woldegiorgis, et al., 2007a)

\begin{tabular}{lcr}
\hline & Chlorhexidine (55-56-1) & \\
\hline Matrix & $\min$ & Nr.S \\
& $\max$ & \\
Biota & $A D$ & 7 \\
Sediment & nd & 7 \\
STP sludge & nd & 11 \\
Farm soil & nd & 3 \\
Effluent & nd & 16 \\
STP influent & nd & 10 \\
Surface water & nd & 12 \\
\hline
\end{tabular}

\section{Reference:}

- (Törneman, 2011a)

\begin{tabular}{|c|c|c|c|c|c|}
\hline & $\begin{array}{r}\text { Florfenicol } \\
(73231-34-2)\end{array}$ & $\begin{array}{l}\text { Phenoxymethyl- } \\
\text { penicillin (87-08-1) }\end{array}$ & $\begin{array}{l}\text { Sulfatroxazol } \\
(23256-23-7)\end{array}$ & $\begin{array}{r}\text { Tiamulin } \\
(55297-95-5)\end{array}$ & \\
\hline \multirow[t]{3}{*}{ Matrix } & $\min$ & $\min$ & $\min$ & $\min$ & Nr.S \\
\hline & $\max$ & $\max$ & $\max$ & $\max$ & \\
\hline & $A D$ & $A D$ & $A D$ & $A D$ & \\
\hline STP sludge $(\mu \mathrm{g} / \mathrm{kg} \mathrm{dw})$ & nd & nd & nd & nd & 10 \\
\hline \multirow[t]{3}{*}{ Surface water $(\mu \mathrm{g} / \mathrm{l})$} & nd & nd & nd & nd & 16 \\
\hline & 0,22 & & & & \\
\hline & 0,1 & & & & \\
\hline Effluent ( $\mu \mathrm{g} / \mathrm{l})$ & nd & nd & nd & nd & 9 \\
\hline STP influent $(\mu \mathrm{g} / \mathrm{l})$ & nd & nd & nd & nd & 9 \\
\hline Pig slurry & nd & nd & nd & nd & 4 \\
\hline Groundwater & nd & nd & nd & nd & 3 \\
\hline
\end{tabular}




\begin{tabular}{|c|c|c|c|c|c|}
\hline & $\begin{array}{r}\text { Florfenicol } \\
(73231-34-2)\end{array}$ & $\begin{array}{l}\text { Phenoxymethyl- } \\
\text { penicillin (87-08-1) }\end{array}$ & $\begin{array}{l}\text { Sulfatroxazol } \\
(23256-23-7)\end{array}$ & $\begin{array}{r}\text { Tiamulin } \\
(55297-95-5)\end{array}$ & \\
\hline $\begin{array}{l}\text { Warning system for } \\
\text { pesticide leaching to } \\
\text { groundwater ( } \mathrm{mg} / \mathrm{kg} \mathrm{dw} \text { ) }\end{array}$ & nd & nd & nd & $\begin{array}{r}\text { nd } \\
0,21 \\
0,21\end{array}$ & 8 \\
\hline Drain water & nd & nd & nd & nd & 1 \\
\hline
\end{tabular}

\section{Reference:}

- (Mogensen, et al., 2008)

\begin{tabular}{llc}
\hline & Ketoconazol (65277-42-1) & \\
\hline Matrix & $\min$ & Nr.S \\
& $\max$ & \\
\multirow{2}{*}{ Effluent } & $A D$ & 7 \\
\hline
\end{tabular}

\section{Reference:}

- (Kjølholt, et al., 2003)

\begin{tabular}{lcc}
\hline & \multicolumn{2}{c}{ Meclocycline (2013-58-3) } \\
\hline Matrix & $\min$ & Nr.S \\
& $\max$ & \\
STPsludge & $A D$ & 7 \\
Effluent & nd & 7 \\
\hline
\end{tabular}

\section{Reference:}

- (Thomas, et al., 2007)

\begin{tabular}{|c|c|c|c|c|c|}
\hline & $\begin{array}{c}\text { Cefalotin } \\
(153-61-7)\end{array}$ & $\begin{array}{r}\text { Cefotaxime } \\
(63527-52-6)\end{array}$ & $\begin{array}{r}\text { Meropenem } \\
8119478-56-7)\end{array}$ & $\begin{array}{r}\text { Pivmecillinam } \\
(33817-20-8)\end{array}$ & \\
\hline \multirow[t]{3}{*}{ Matrix } & $\min$ & $\min$ & $\min$ & $\min$ & Nr.S \\
\hline & $\max$ & $\max$ & $\max$ & $\max$ & \\
\hline & $A D$ & $A D$ & $A D$ & $A D$ & \\
\hline Biota & nd & nd & nd & & 3 \\
\hline Sediment & nd & nd & nd & & 6 \\
\hline STP sludge (ng/g dw) & nd & nd & nd & & 4 \\
\hline \multirow[t]{3}{*}{ Effluent (ng/l) } & nd & nd & nd & & 10 \\
\hline & & 577 & & & \\
\hline & & 187,3 & & & \\
\hline \multirow[t]{3}{*}{ Surface water (ng/l) } & nd & nd & nd & & 12 \\
\hline & & 441 & & & \\
\hline & & 167 & & & \\
\hline
\end{tabular}

\section{Reference:}

- (Schlabach, et al., 2009) 


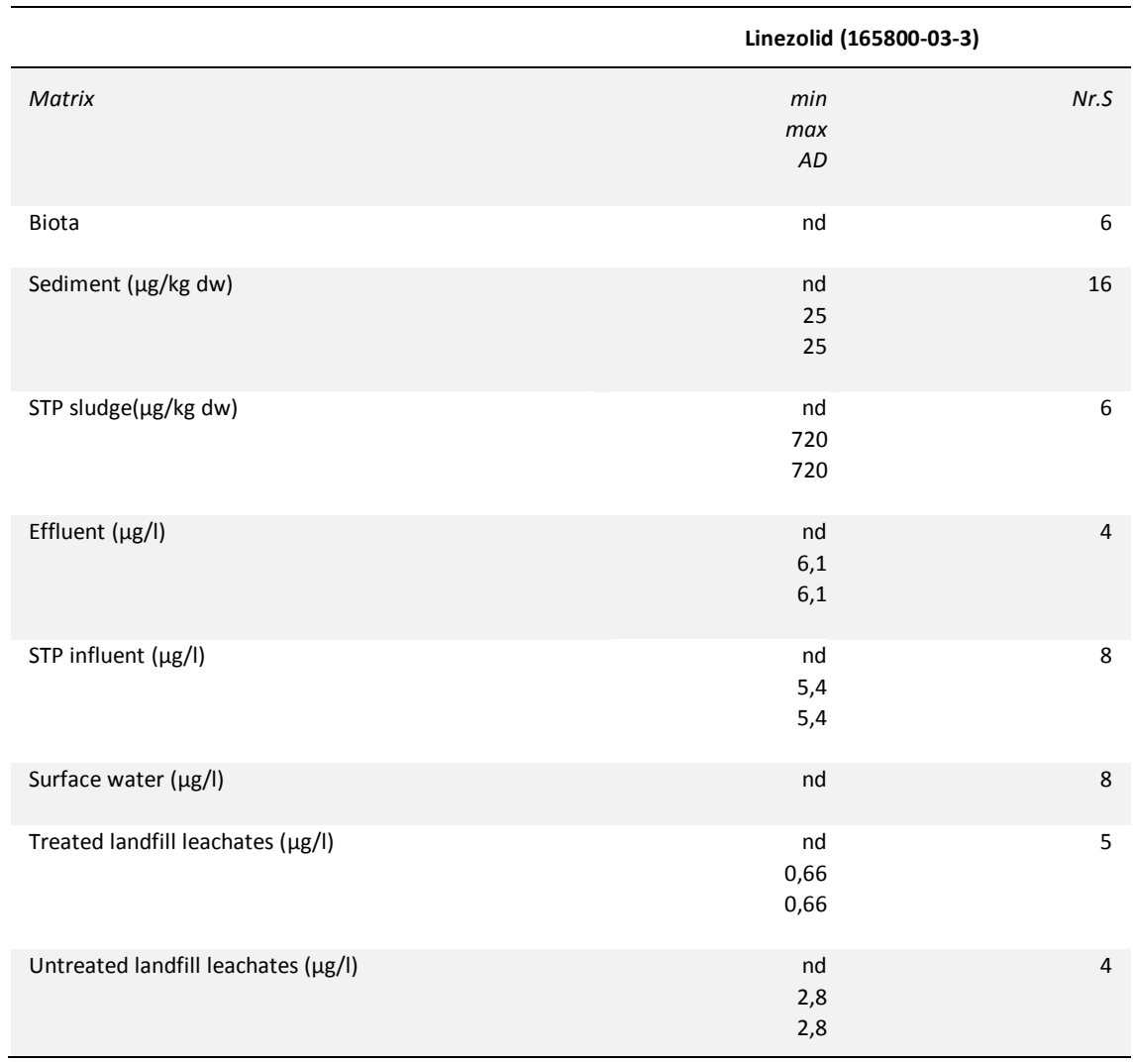

\section{Reference:}

- (Møskeland, et al., 2006) 



\section{Hormones and hormone antagonists}

\subsection{Scope and definition}

This category includes natural and synthetic hormones as well as hormone antagonists. The antagonists are typically active ingredients used in the treatment of prostatic and breast cancer.

These compounds have an endocrine disruption effect, that may affect wild species when released to the environment.

Table 3 presents hormones and hormone antagonists identified in monitoring studies the Nordic countries.

Table 3: Hormones and hormone antagonists monitored in the Nordic countries

\begin{tabular}{ll}
\hline Compounds monitored & CAS nr \\
\hline Estradiol & $50-28-2$ \\
Estriol (hormone) & $50-27-1$ \\
Estrone (hormone) & $53-16-7$ \\
Ethinylestradiol (hormone) & $57-63-6$ \\
Etonogestrel (progesterone) & $54048-10-1$ \\
Finasteride (synthetic antiandrogen) & $98319-26-7$ \\
Flutamide, antiandrogen (antagonist) & $13311-84-7$ \\
Levonorgestrel (progestogen) & $797-63-7$ \\
Norethindrone (progestogen) & $68-22-4$ \\
Progesterone (hormone) & $57-83-0$ \\
Tamoxifen (antagonist of estrogen) & $10540-29-1$ \\
\hline
\end{tabular}

\subsection{Compounds mapped}

\subsubsection{Estradiol (17 $\beta$-estradiol)}

CAS nr.: 50-28-2

Info: Estradiol (E2 or $17 \beta$-estradiol, also oestradiol) is a sex hormone. It is also a metabolite of testosterone.

Toxicity data:

LOEL (Oryzias latipes) $=0,004 \mu \mathrm{g} / \mathrm{l}$

NOEL (Oryzias latipes) induced intersex (testis-ova) $=0,0004 \mu \mathrm{g} / \mathrm{l}$ (Andersson, et al., 2006)

$N E C=0,00002 \mu \mathrm{g} / \mathrm{l}$ (Schlabach, et al., 2007) 

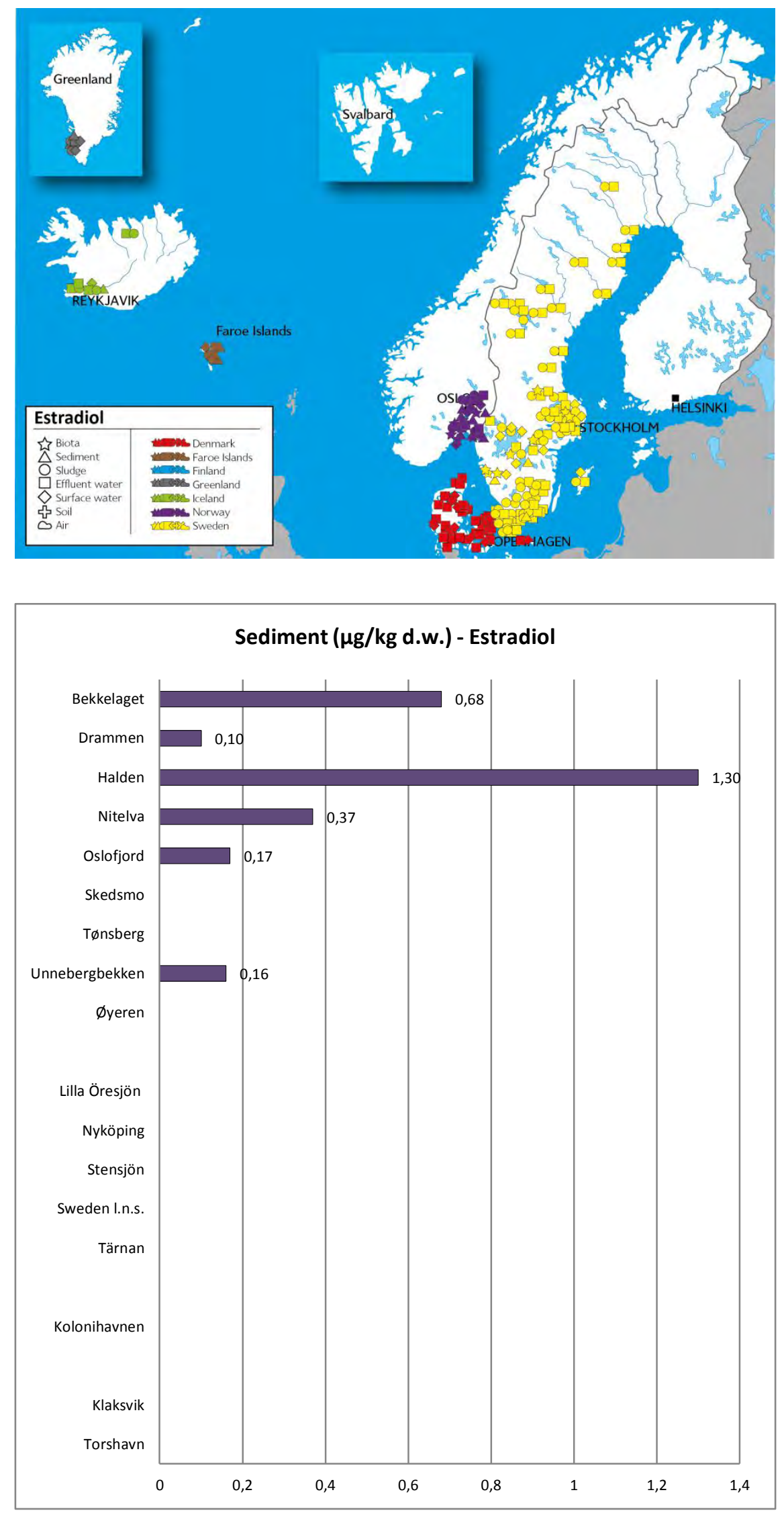


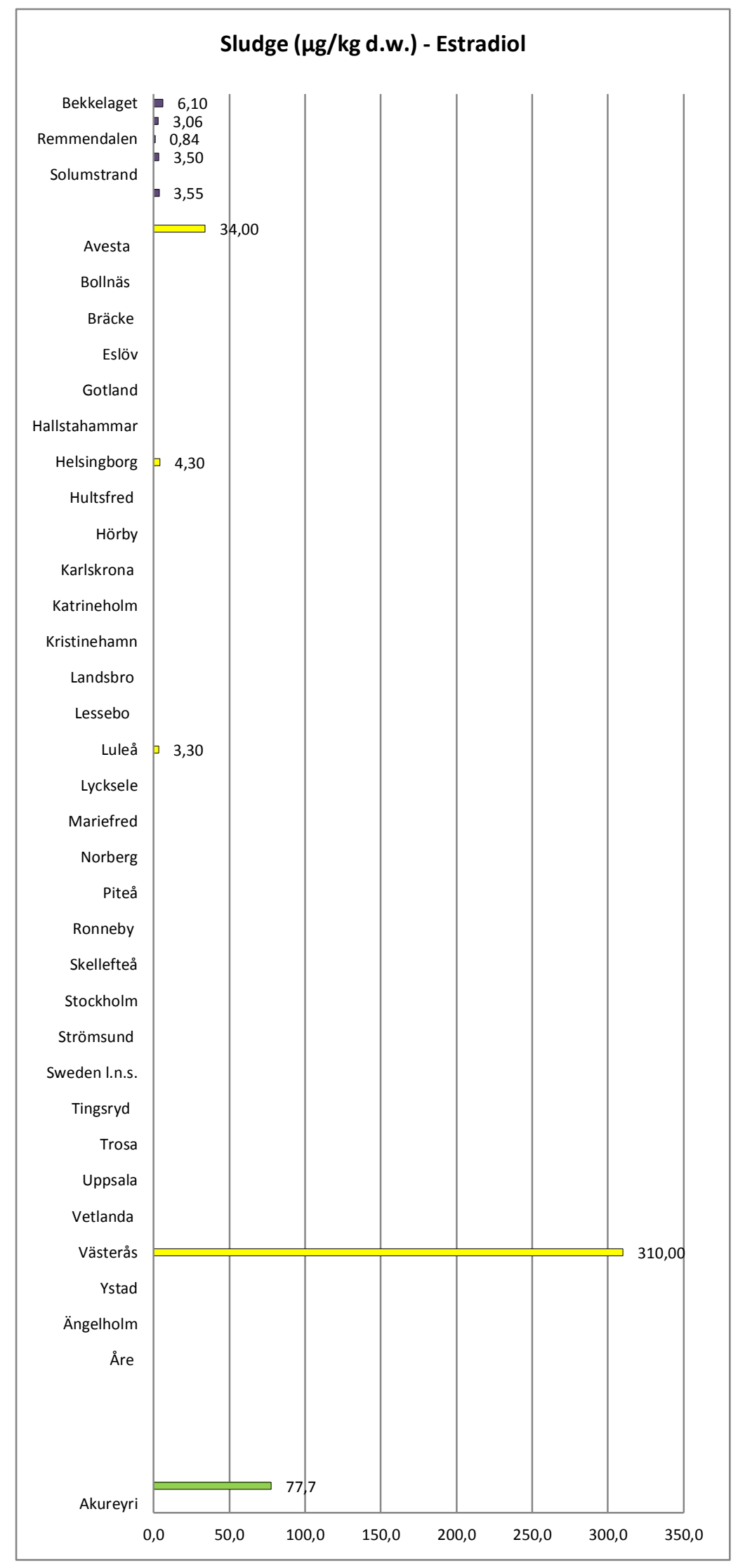




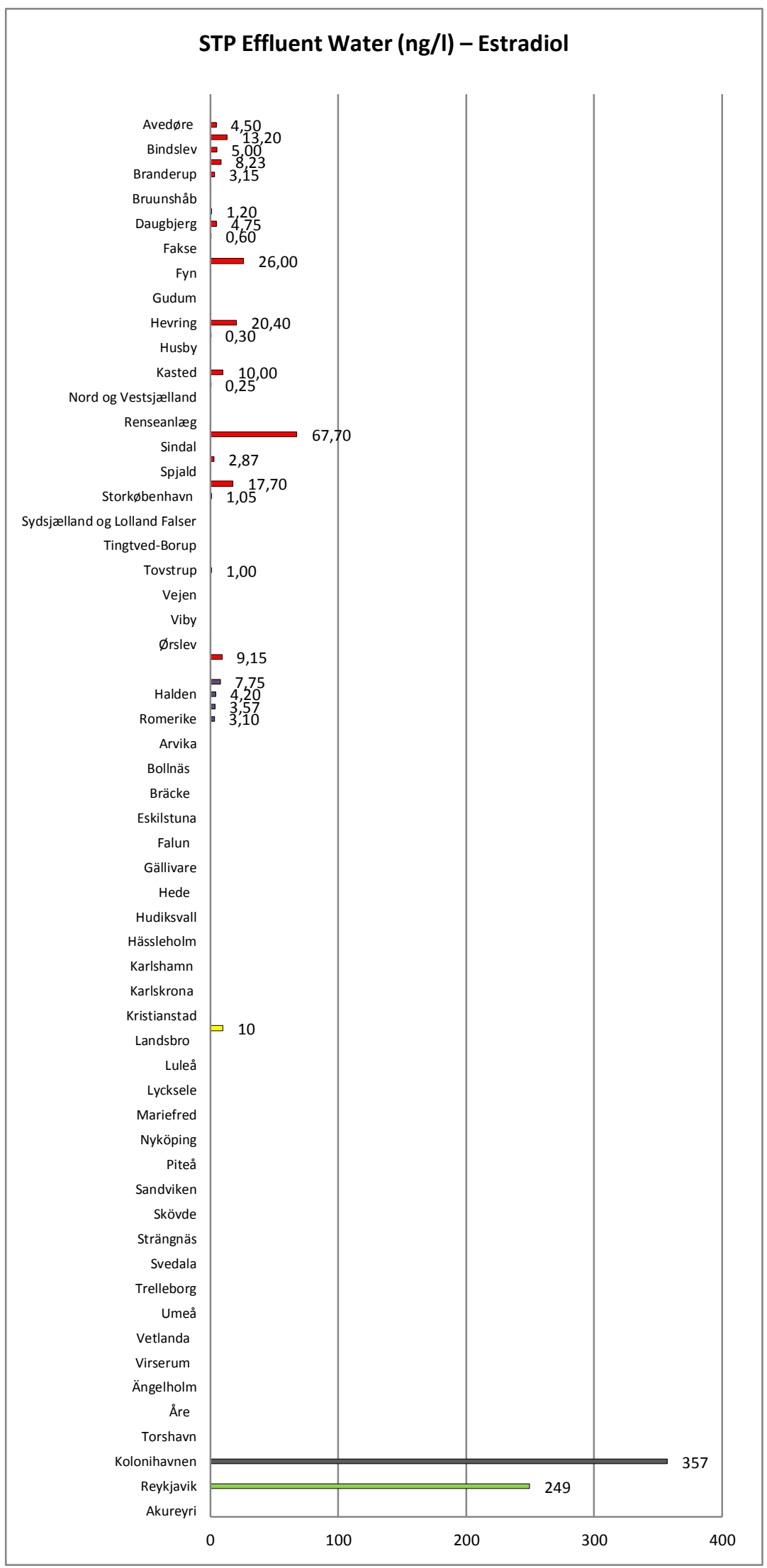




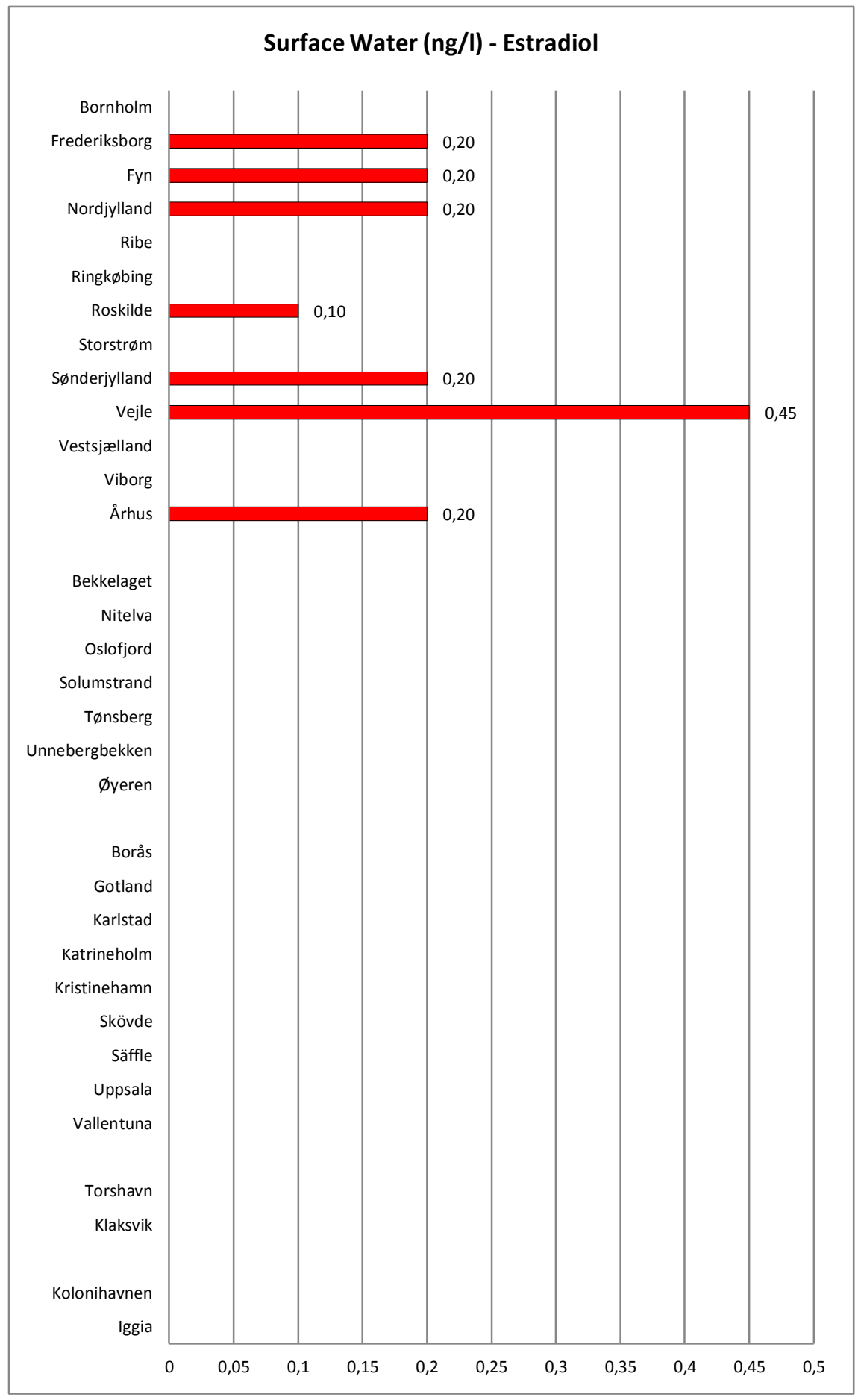




\begin{tabular}{|c|c|c|c|c|c|c|c|c|c|c|c|c|}
\hline \multirow{2}{*}{$\begin{array}{l}\text { Estradiol } \\
\text { Matrix }\end{array}$} & \multicolumn{2}{|c|}{ Norway } & \multicolumn{2}{|c|}{ Denmark } & \multicolumn{2}{|c|}{ Sweden } & \multicolumn{2}{|c|}{ Faroe Islands (h) } & \multicolumn{2}{|c|}{ Greenlan (h) } & \multicolumn{2}{|c|}{ Iceland (h) } \\
\hline & $\begin{array}{l}\min \\
\max \\
A D\end{array}$ & $\begin{array}{l}\text { Nr.S } \\
\text { (ref) }\end{array}$ & $\begin{array}{r}\min \\
\max \\
A D\end{array}$ & $\begin{array}{l}\text { Nr.S } \\
\text { (ref) }\end{array}$ & $\begin{array}{r}\min \\
\max \\
A D\end{array}$ & $\begin{array}{l}\text { Nr.S } \\
\text { (ref) }\end{array}$ & $\begin{array}{r}\min \\
\max \\
A D\end{array}$ & $\begin{array}{l}N r . S \\
\text { (ref) }\end{array}$ & $\begin{array}{r}\min \\
\max \\
A D\end{array}$ & $\begin{array}{l}\text { Nr.S } \\
\text { (ref) }\end{array}$ & $\begin{array}{r}\min \\
\max \\
A D\end{array}$ & $\begin{array}{l}\text { Nr.S } \\
\text { (ref) }\end{array}$ \\
\hline Biota ( $\mu \mathrm{g} / \mathrm{kg}$ ww) & nd & 6 (a) & & & nd & $18(\mathrm{fg})$ & & & & & & \\
\hline STP sludge $(\mu \mathrm{g} / \mathrm{kg} \mathrm{dw})$ & $\begin{array}{r}\text { nd } \\
7 \\
3,28\end{array}$ & $13(a b)$ & & & $\begin{array}{r}\text { nd } \\
310 \\
87,90\end{array}$ & $66(f g)$ & nd & 2 & nd & 2 & $\begin{array}{r}\text { nd } \\
77,7 \\
77,7\end{array}$ & 3 \\
\hline Sediments $(\mu \mathrm{g} / \mathrm{kg} \mathrm{dw})$ & $\begin{array}{r}\text { nd } \\
1,30 \\
0,46\end{array}$ & $12(a)$ & & & nd & $7(f)$ & nd & 3 & nd & 2 & & \\
\hline $\begin{array}{l}\text { Landfild leachate } \\
\text { sediments ( } \mu \mathrm{g} / \mathrm{kg} \mathrm{dw})\end{array}$ & $\begin{array}{r}\text { nd } \\
0,77 \\
0,77\end{array}$ & 4 (a) & & & & & & & & & & \\
\hline $\begin{array}{l}\text { Untreated landfill } \\
\text { leachates (ng/l) }\end{array}$ & $\begin{array}{r}\text { nd } \\
5 \\
3,83\end{array}$ & 4 (a) & & & nd & $7(f)$ & & & & & & \\
\hline $\begin{array}{l}\text { Treated landfill } \\
\text { leachates (ng/l) }\end{array}$ & $\begin{array}{r}\text { nd } \\
3 \\
2,63\end{array}$ & 5 (a) & & & & & & & & & & \\
\hline Surface water (ng/l) & $\begin{array}{r}\text { nd } \\
0,25 \\
1,19\end{array}$ & $8(a)$ & $\begin{array}{r}\text { nd } \\
0,70 \\
0,26\end{array}$ & 124 (c) & $\begin{array}{r}\text { nd } \\
2 \\
2\end{array}$ & $32(\mathrm{fg})$ & nd & 5 & nd & 5 & & \\
\hline STP Influent (ng/l) & $\begin{array}{r}\text { nd } \\
15,50 \\
8,54\end{array}$ & 8 (a) & $\begin{array}{r}0,10 \\
25,5 \\
10,29\end{array}$ & 14 (c) & $\begin{array}{r}\text { nd } \\
1,20 \\
1,20\end{array}$ & $31(\mathrm{fg})$ & $\begin{array}{r}\text { nd } \\
465 \\
465\end{array}$ & 2 & & & nd & 5 \\
\hline STP effluent (ng/l) & $\begin{array}{r}\text { nd } \\
7,75 \\
4,29\end{array}$ & $11(a b)$ & $\begin{array}{r}\text { nd } \\
67,7 \\
8,16\end{array}$ & $\begin{array}{r}100 \\
\text { (cde) }\end{array}$ & $\begin{array}{l}\text { nd } \\
10 \\
10\end{array}$ & $68(\mathrm{fg})$ & nd & 2 & $\begin{array}{l}357 \\
357 \\
357\end{array}$ & 1 & $\begin{array}{r}\text { nd } \\
249 \\
249\end{array}$ & 5 \\
\hline Drinking water (ng/l) & & & & & nd & 6 (g) & & & & & & \\
\hline Manure & & & & & nd & $5(f)$ & & & & & & \\
\hline Hospital WW (ng/l) & & & & & nd & $7(f)$ & nd & 2 & $\begin{array}{r}342 \\
375 \\
358,4\end{array}$ & 2 & & \\
\hline Miscellaneous ${ }^{67}(\mathrm{ng} / \mathrm{l})$ & & & $\begin{array}{l}0,10 \\
23,3 \\
1,89\end{array}$ & 107 (c) & & & & & & & & \\
\hline
\end{tabular}

\section{References:}

a) (Møskeland, et al., 2006)

b) (Thomas, et al., 2007)

c) (Stuer-Lauridsen, et al., 2005)

d) (Kjølholt, et al., 2003)

e) (Ingerslev, et al., 2003)

f) (Andersson, et al., 2006) 
g) (Fick, et al., 2011)

h) (Huber, et al., NA)

\section{Conclusion:}

The compound has been monitored in two studies in Norway, three studies in Denmark, two studies in Sweden and one study for Faroe Islands, Greenland and Iceland.

Estradiol has been detected in several matrices:

- STP sludge in Norway, Sweden and Iceland. The lowest values were detected in Norway. The highest values were detected in Sweden, five times higher than in Iceland and about fourty times higher than in Norway

- Sediment in Norway only

- Surface water only in Norway, Denmark, and Sweden in concentration above the PNEC

- STP effluent water, in all the countries except Faroe Island. The lowest values were detected in Norway. The concentration in Denmark was almost ten times higher than in Norway. The highest concentration was detected in Greenland and Iceland. All detected values were above PNEC value

- STP influent in Norway, Denmark and Sweden. The lowest concentration was detected in Sweden while the highest in Faroe Islands

- Hospital wastewater in similar concentration only in Greenland 


\subsubsection{Estriol}

CAS nr.: 50-27-1

Info: Extrogen hormone antagonist for estradiol

Toxicity data: LOEL (Oryzias latipes) induced intersex (testis-ova), exposure from hatching, 90 days $=0.75 \mu \mathrm{g} / \mathrm{l}$

(Andersson, et al., 2006)

$P N E C=0,00075 \mu / 1$ (Schlabach, et al., 2007)

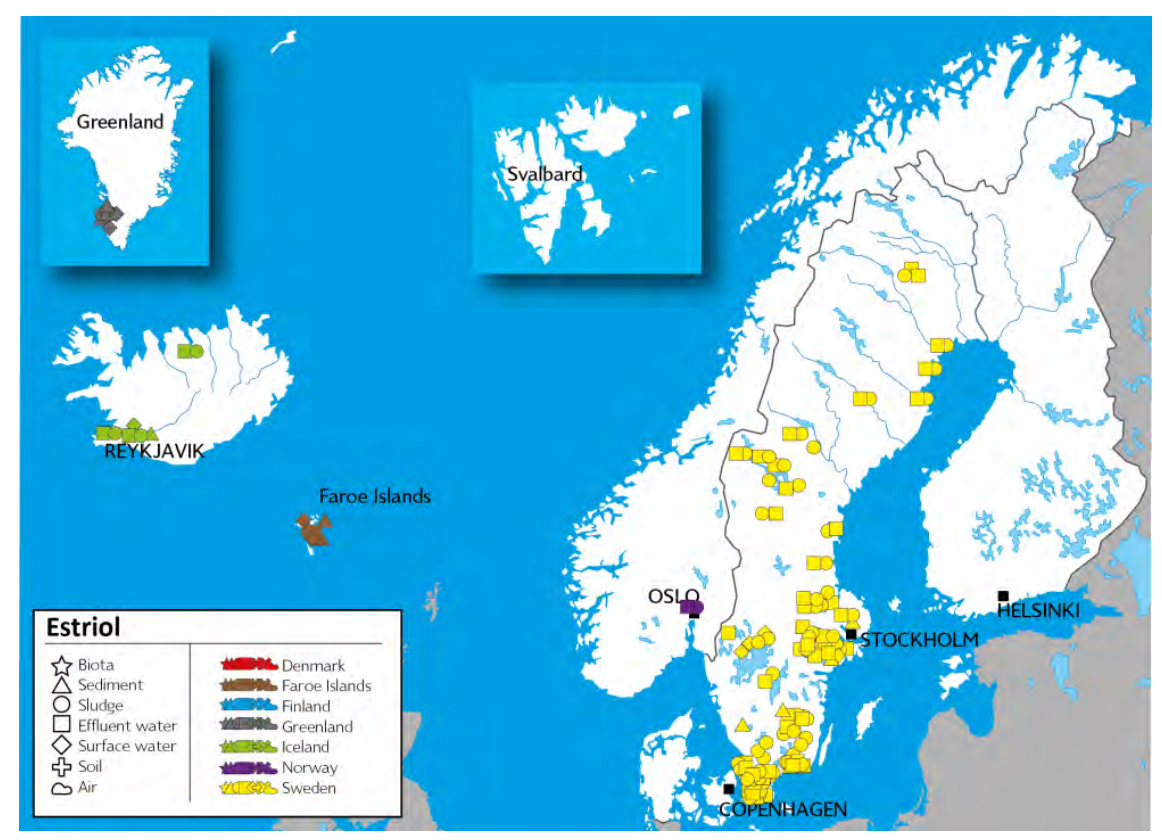




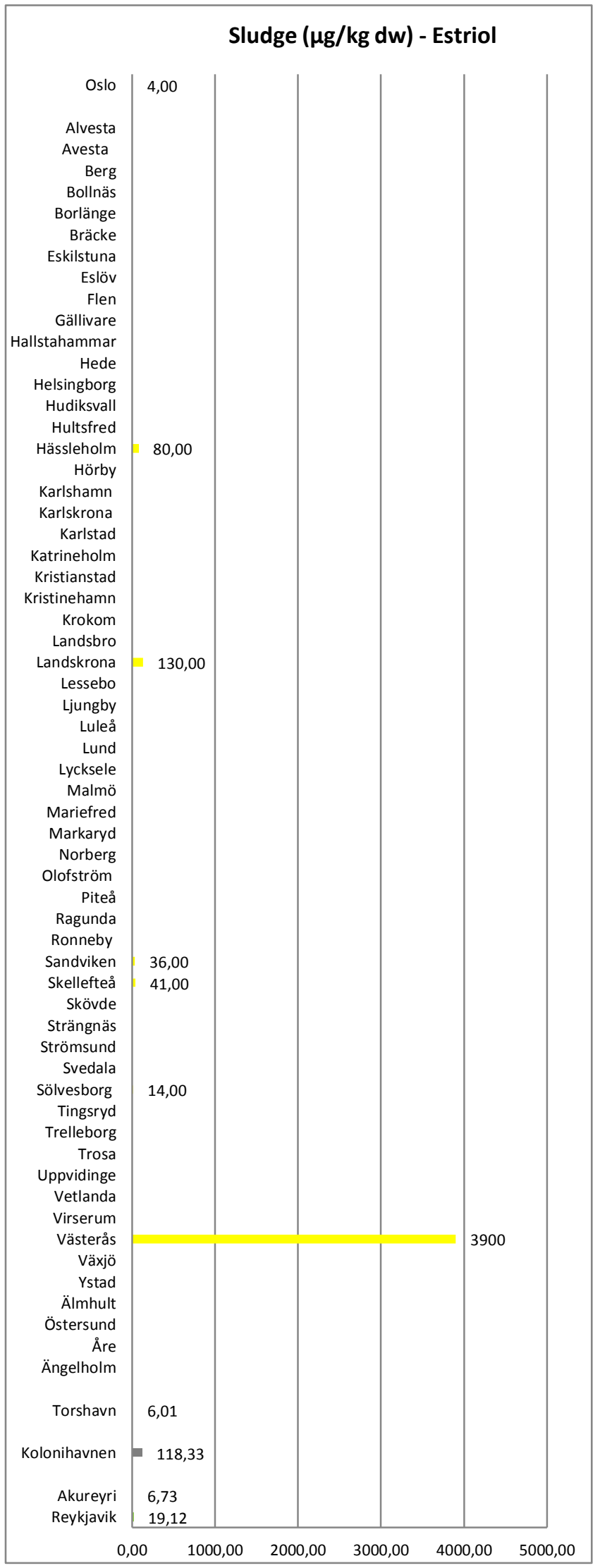




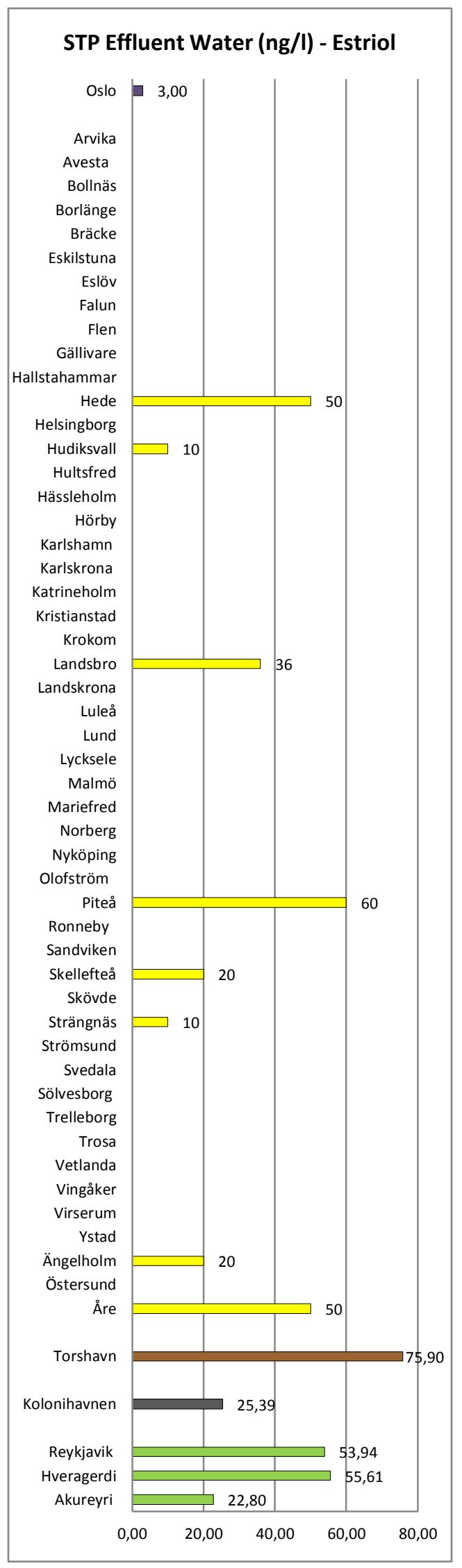




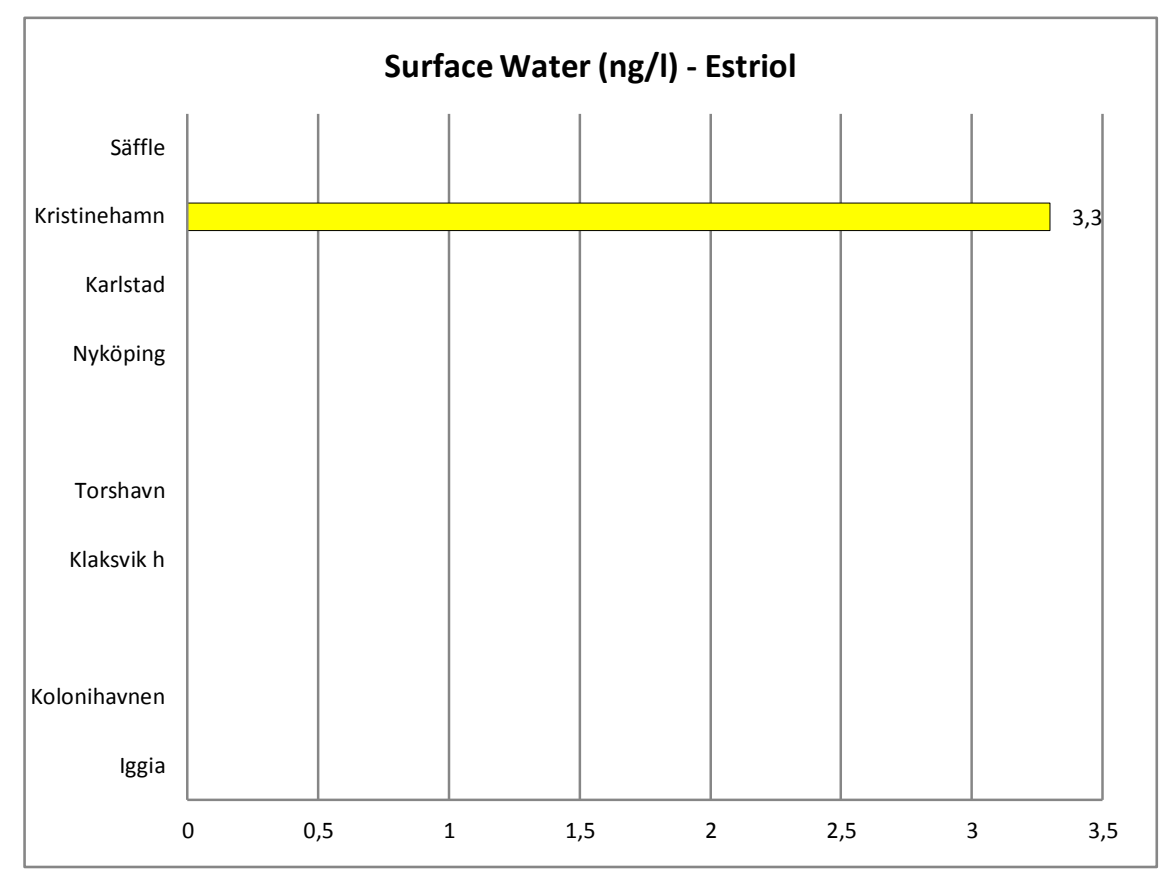

\begin{tabular}{|c|c|c|c|c|c|c|c|c|c|c|}
\hline \multirow{2}{*}{$\frac{\text { Estriol }}{\text { Matrix }}$} & \multicolumn{2}{|c|}{ Norway (a) } & \multicolumn{2}{|c|}{ Sweden(b) } & \multicolumn{2}{|c|}{ Faroe Islands (c) } & \multicolumn{2}{|c|}{ Greenland (c) } & \multicolumn{2}{|c|}{ Iceland (c) } \\
\hline & $\begin{array}{r}\min \\
\max \\
A D\end{array}$ & $\begin{array}{l}\text { Nr.S } \\
\text { (ref) }\end{array}$ & $\begin{array}{r}\min \\
\max \\
A D\end{array}$ & $\begin{array}{l}\text { Nr.S } \\
\text { (ref) }\end{array}$ & $\begin{array}{r}\min \\
\max \\
A D\end{array}$ & $\begin{array}{l}\text { Nr.S } \\
\text { (ref) }\end{array}$ & $\begin{array}{r}\min \\
\max \\
A D\end{array}$ & $\begin{array}{l}\text { Nr.S } \\
\text { (ref) }\end{array}$ & $\begin{array}{r}\min \\
\max \\
A D\end{array}$ & $\begin{array}{l}\text { Nr.S } \\
\text { (ref) }\end{array}$ \\
\hline Biota $(\mu \mathrm{g} / \mathrm{kg} w w)$ & & & nd & 3 & & & & & & \\
\hline $\begin{array}{l}\text { STP sludge }(\mu \mathrm{g} / \mathrm{kg} \\
\mathrm{dw})\end{array}$ & $\begin{array}{r}\text { nd } \\
4 \\
4\end{array}$ & 7 & $\begin{array}{r}\text { nd } \\
3900 \\
700,17\end{array}$ & 61 & $\begin{array}{l}5,59 \\
6,42 \\
6,01\end{array}$ & 2 & $\begin{array}{r}26,7 \\
210 \\
118\end{array}$ & 2 & $\begin{array}{r}\text { nd } \\
19,1 \\
12,93\end{array}$ & 3 \\
\hline Sediment $(\mu \mathrm{g} / \mathrm{kg} \mathrm{dw})$ & & & nd & 8 & nd & 3 & nd & 2 & & \\
\hline $\begin{array}{l}\text { Landfill leachates, } \\
\text { water (ng/l) }\end{array}$ & & & $\begin{array}{l}\text { nd } \\
20 \\
20\end{array}$ & 7 & & & & & & \\
\hline Surface water (ng/l) & & & $\begin{array}{r}\text { nd } \\
3,3 \\
3,3\end{array}$ & 19 & nd & 5 & nd & 5 & & \\
\hline STP Influent (ng/l) & & & $\begin{array}{l}\text { nd } \\
24 \\
24\end{array}$ & 19 & $\begin{array}{l}58,8 \\
58,8 \\
58,8\end{array}$ & 2 & & & $\begin{array}{r}\text { nd } \\
98,7 \\
85,9\end{array}$ & 5 \\
\hline STP effluent (ng/l) & $\begin{array}{r}\text { nd } \\
3 \\
3\end{array}$ & 7 & $\begin{array}{l}\text { nd } \\
60 \\
32\end{array}$ & 60 & $\begin{array}{l}75,9 \\
75,9 \\
75,9\end{array}$ & 1 & $\begin{array}{l}25,4 \\
25,4 \\
25,4\end{array}$ & 1 & $\begin{array}{r}\text { nd } \\
53,9 \\
47,0\end{array}$ & 5 \\
\hline Manure $(\mu \mathrm{g} / \mathrm{kg} \mathrm{dw})$ & & & $\begin{array}{r}\text { nd } \\
180 \\
180\end{array}$ & 4 & & & & & & \\
\hline Hospital WW (ng/I) & & & $\begin{array}{l}\text { nd } \\
50 \\
50\end{array}$ & 7 & $\begin{array}{r}31 \\
198 \\
114,6\end{array}$ & 2 & $\begin{array}{l}135 \\
140 \\
137\end{array}$ & 2 & & \\
\hline
\end{tabular}




\section{References:}

a) (Thomas, et al., 2007)

b) (Andersson, et al., 2006)

c) (Fick, et al., 2011)

d) (Huber, et al., NA)

\section{Conclusion:}

The compound was investigated in one study in Norway, one in Sweden and one in Faroe Islands, Greenland and Iceland.

It was detected in several matrices:

- STP sludge in Sweden, Faroe Islands, Greenland and Iceland. Results from the different countries show a high variability. The concentration detected in Sweden are above twenty times higher than in Greenland and two hundred times higher than in Iceland

- STP effluent water in all countries. The lowest concentration was detected in Norway. High values were detected in Greenland. All the detected values were above the PNEC

- STP influent water in Sweden (lowest concentrations), Faroe Islands (two times higher than Sweden) and in Iceland (four times higher than Sweden)

- Surface water only in Sweden in values were above the PNEC

- Hospital waste water in Faroe Islands and Greenland. The highest values were detected in Greenland

- Landfill leachate and manure in Sweden 


\subsubsection{Estrone}

CAS nr.: 53-16-7

Info: Estrogenic hormone

Toxicity data: NA

$P N E C=\mathrm{NA}$
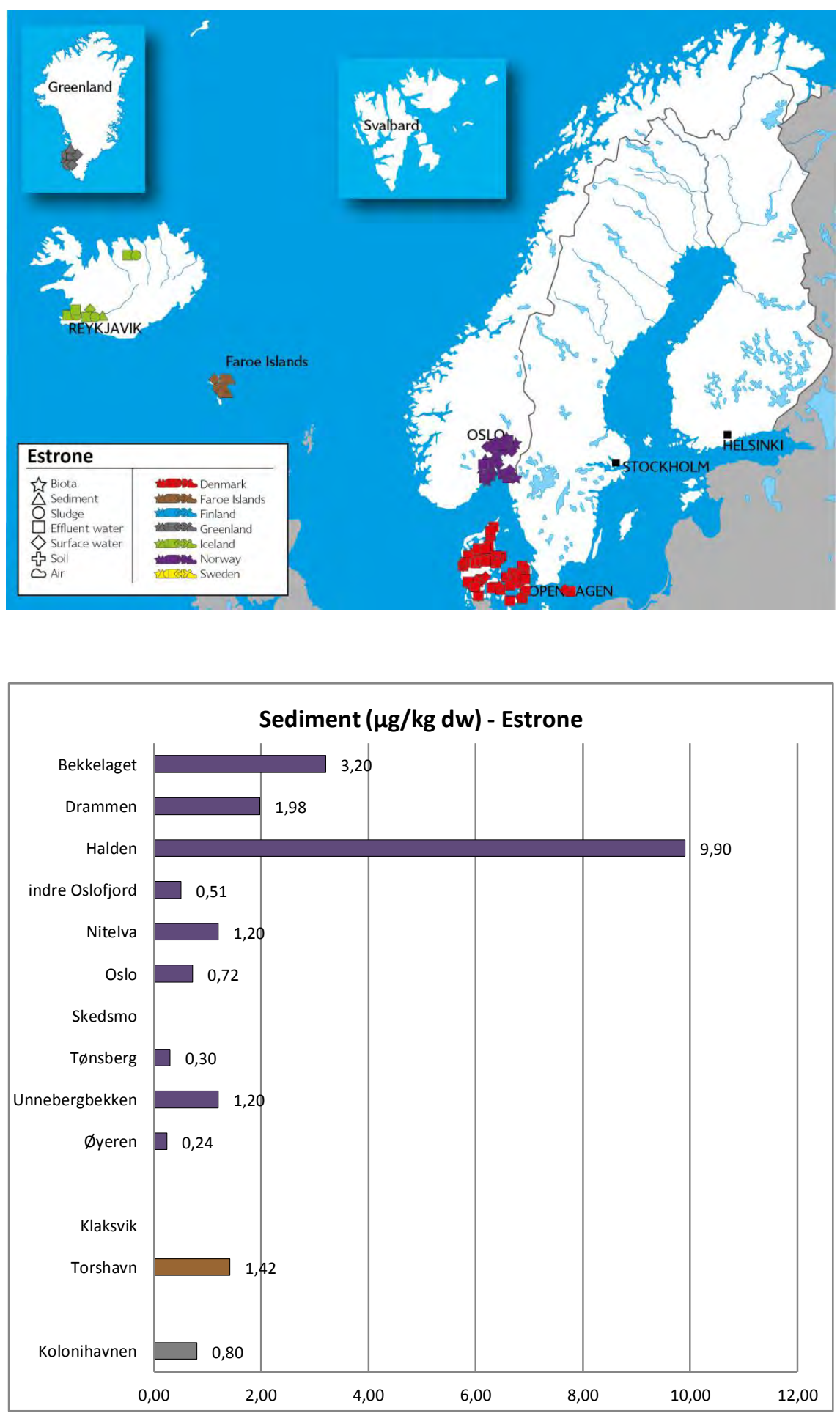


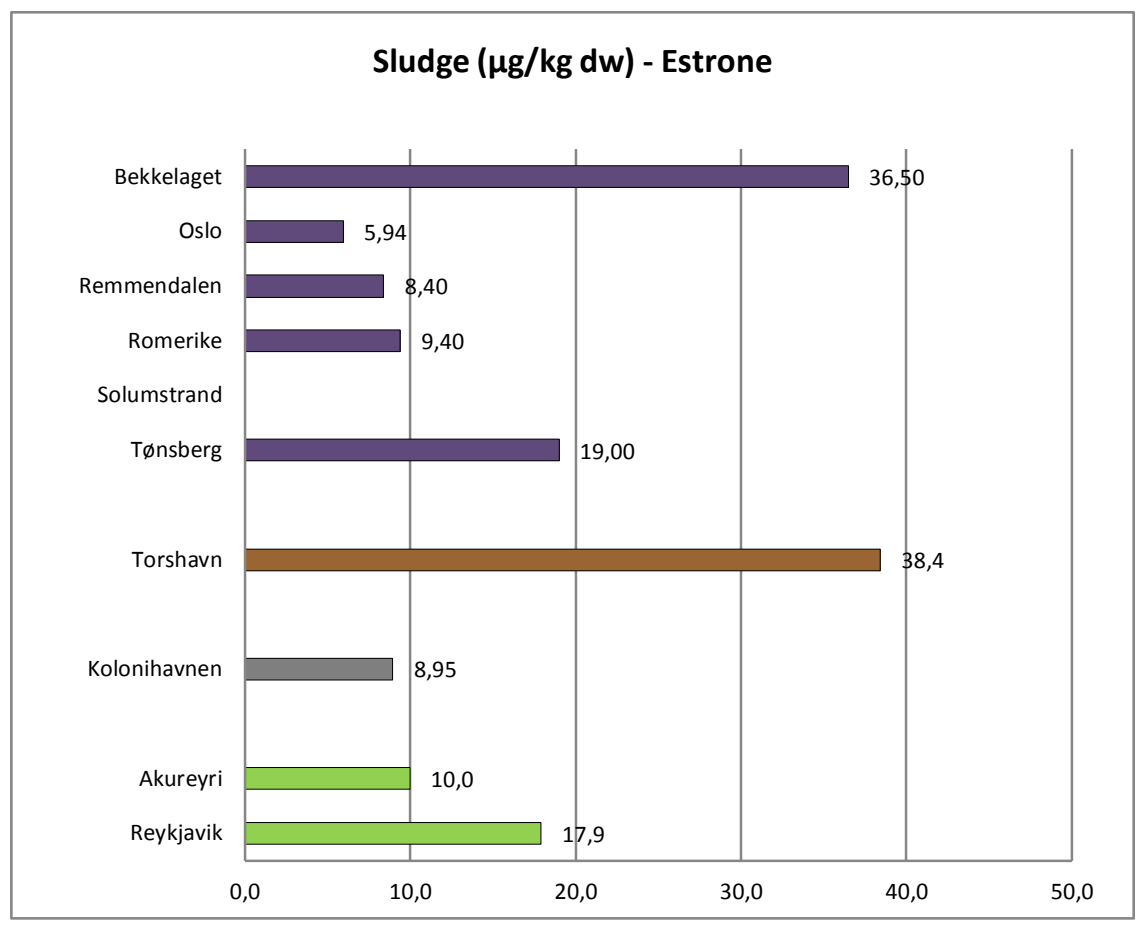




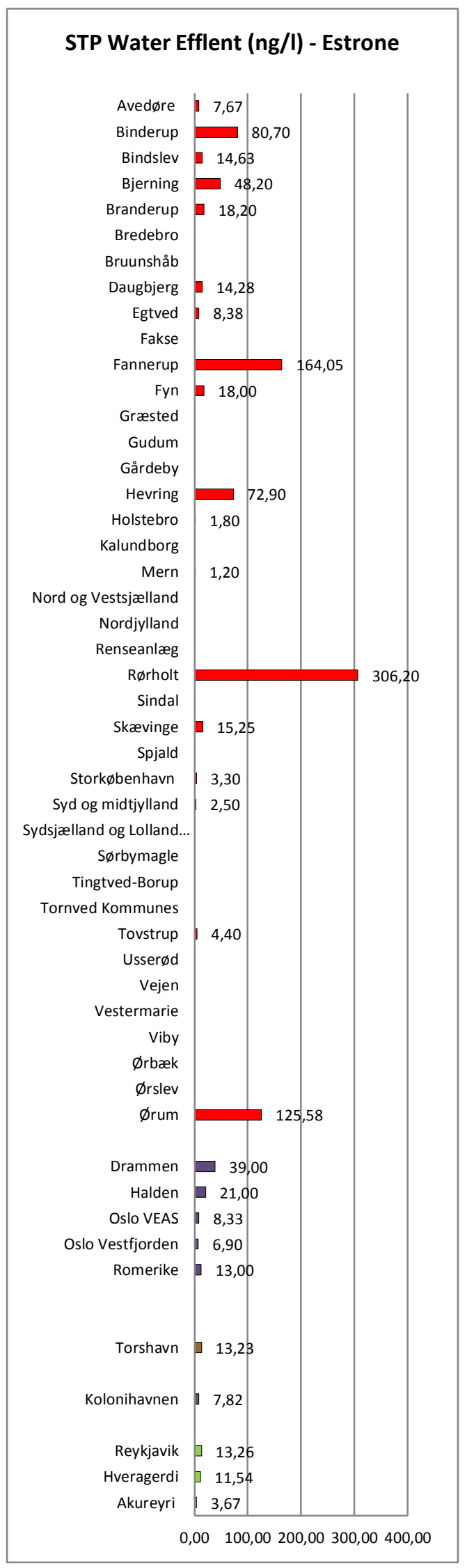




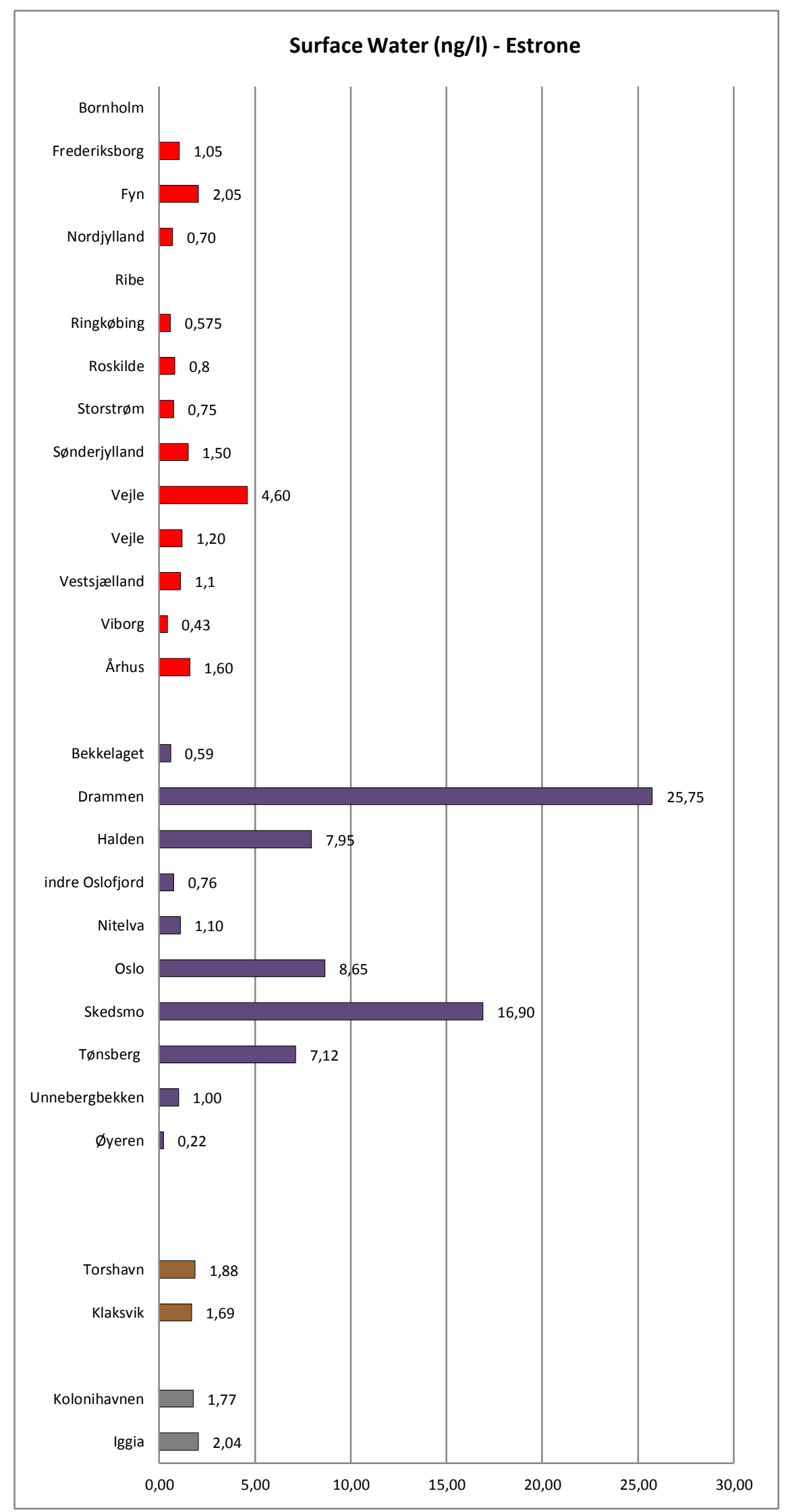




\begin{tabular}{|c|c|c|c|c|c|c|c|c|c|c|}
\hline \multirow{2}{*}{$\begin{array}{l}\text { Estrone } \\
\text { Matrix }\end{array}$} & \multicolumn{2}{|c|}{ Norway } & \multicolumn{2}{|c|}{ Denmark } & \multicolumn{2}{|c|}{ Faroe Islands (f) } & \multicolumn{2}{|c|}{ Greenland (f) } & \multicolumn{2}{|c|}{ Iceland (f) } \\
\hline & $\begin{array}{r}\min \\
\max \\
A D\end{array}$ & $\begin{array}{l}\text { Nr.S } \\
\text { (ref) }\end{array}$ & $\begin{array}{r}\min \\
\max \\
A D\end{array}$ & $\begin{array}{l}\text { Nr.S } \\
\text { (ref) }\end{array}$ & $\begin{array}{r}\min \\
\max \\
A D\end{array}$ & $\begin{array}{l}\text { Nr.S } \\
\text { (ref) }\end{array}$ & $\begin{array}{r}\min \\
\max \\
A D\end{array}$ & $\begin{array}{l}\text { Nr.S } \\
\text { (ref) }\end{array}$ & $\begin{array}{r}\min \\
\max \\
A D\end{array}$ & $\begin{array}{l}\mathrm{Nr} . \mathrm{S} \\
\text { (ref) }\end{array}$ \\
\hline Biota $(\mu \mathrm{g} / \mathrm{kg} w w)$ & nd & 6 (a) & & & & & & & & \\
\hline STP sludge $(\mu \mathrm{g} / \mathrm{kg} \mathrm{dw})$ & $\begin{array}{r}\text { nd } \\
36,50 \\
10,7\end{array}$ & $13(a b)$ & & & $\begin{array}{l}15,4 \\
61,4 \\
38,4\end{array}$ & 2 & $\begin{array}{r}6,89 \\
11 \\
8,95\end{array}$ & 2 & $\begin{array}{r}1,1 \\
18,8 \\
12,6\end{array}$ & 3 \\
\hline Sediment $(\mu \mathrm{g} / \mathrm{kg} \mathrm{dw})$ & $\begin{array}{r}\text { nd } \\
9,90 \\
1,64\end{array}$ & $12(a)$ & & & $\begin{array}{r}\text { nd } \\
1,42 \\
1,42\end{array}$ & 3 & $\begin{array}{r}\text { nd } \\
0,80 \\
0,80\end{array}$ & 2 & & \\
\hline $\begin{array}{l}\text { Landfild leachate } \\
\text { sediment }(\mu \mathrm{g} / \mathrm{kg} \mathrm{dw})\end{array}$ & $\begin{array}{r}\text { nd } \\
4,9 \\
2,07\end{array}$ & 4 (a) & & & & & & & & \\
\hline $\begin{array}{l}\text { Untreated landfill } \\
\text { leachates (ng/l) }\end{array}$ & $\begin{array}{r}4,3 \\
50 \\
16,08\end{array}$ & 5 (a) & & & & & & & & \\
\hline $\begin{array}{l}\text { Treated landfill leacha- } \\
\text { tes (ng/l) }\end{array}$ & $\begin{array}{r}6,3 \\
26 \\
14,32\end{array}$ & 4 (a) & & & & & & & & \\
\hline Surface water (ng/l) & $\begin{array}{r}0,22 \\
50 \\
7\end{array}$ & $8(a b)$ & $\begin{array}{r}\text { nd } \\
6,4 \\
1,25\end{array}$ & 124 (c) & $\begin{array}{r}\text { nd } \\
2,01 \\
1,83\end{array}$ & 5 & $\begin{array}{r}\text { nd } \\
2,09 \\
1,91\end{array}$ & 5 & & \\
\hline STP Influent (ng/l) & $\begin{array}{r}10 \\
52 \\
31,5\end{array}$ & 8 (a) & $\begin{array}{r}\text { nd } \\
131 \\
42\end{array}$ & 14 (c) & $\begin{array}{l}8,95 \\
11,6 \\
10,2\end{array}$ & 2 & & & $\begin{array}{l}2,04 \\
20,9 \\
9,91\end{array}$ & 5 \\
\hline STP effluent (ng/l) & $\begin{array}{r}\text { nd } \\
39 \\
14,98\end{array}$ & $11(a b)$ & $\begin{array}{r}\text { nd } \\
310 \\
40\end{array}$ & 100 (cde) & $\begin{array}{l}13,2 \\
13,2 \\
13,2\end{array}$ & 1 & $\begin{array}{l}7,82 \\
7,82 \\
7,82\end{array}$ & 1 & $\begin{array}{r}3,6 \\
13,3 \\
8,7\end{array}$ & 5 \\
\hline Hospital WW (ng/l) & & & & & $\begin{array}{r}7,44 \\
18,5 \\
12,97\end{array}$ & 2 & $\begin{array}{r}18,9 \\
21 \\
19,95\end{array}$ & 2 & & \\
\hline Miscellaneous ${ }^{68}(\mathrm{ng} / \mathrm{l})$ & & & $\begin{array}{r}\text { nd } \\
112,4 \\
4,84\end{array}$ & 106 (c) & & & & & & \\
\hline
\end{tabular}

\section{References:}

a) (Møskeland, et al., 2006)

b) (Thomas, et al., 2007)

c) (Stuer-Lauridsen, et al., 2005)

d) (Kjølholt, et al., 2003)

e) (Ingerslev, et al., 2003)

f) (Huber, et al., NA) 


\section{Conclusion:}

Estron has been monitored in two studies in Norway, three studies in Denmark and one study in Faroe Islands, Greenland and Iceland.

The compound were detected in several matrices:

- STP sludge in Norway, Faroe Islands, Greenland and Iceland. The highest values were detected in Faroe Islands

- Sediment in Norway, Faroe Islands and Greenland. The lowest concen-trations were detected in Faroe Island and Greenland whilst the highest were detected in Norway (ten times higher than in the other countries)

- Surface water in Denmark, Faroe Islands and Greenland. Highest values were detected in Norway

- STP effluent water in all the countries. The highest values were detected in Denmark (about ten times higher than in the other countries)

- In STP influent water in Norway, Denmark, Faroe Islands and Iceland. The highest concentration was detected in Denmark

- In hospital waste water in Faroe Islands and Greenland.

- In landfill leachate in Norway

- In miscellaneous samples of water in Denmark

\subsubsection{Ethinylestradiol (17 $\alpha$-ethinylestradiol)}

CAS nr.: 57-63-6

Info: Derivative of estradiol used as orally bio-active estrogen used in almost all modern formulations of combined oral contraceptive pills.

Toxicity data: LOEL (Oryzias latipes)> 0,01 $\mu \mathrm{g} / \mathrm{l}$

(Andersson, et al., 2006)

$P N E C=0,00002 \mathrm{ng} / \mathrm{l}$ (Schlabach, et al., 2007) 

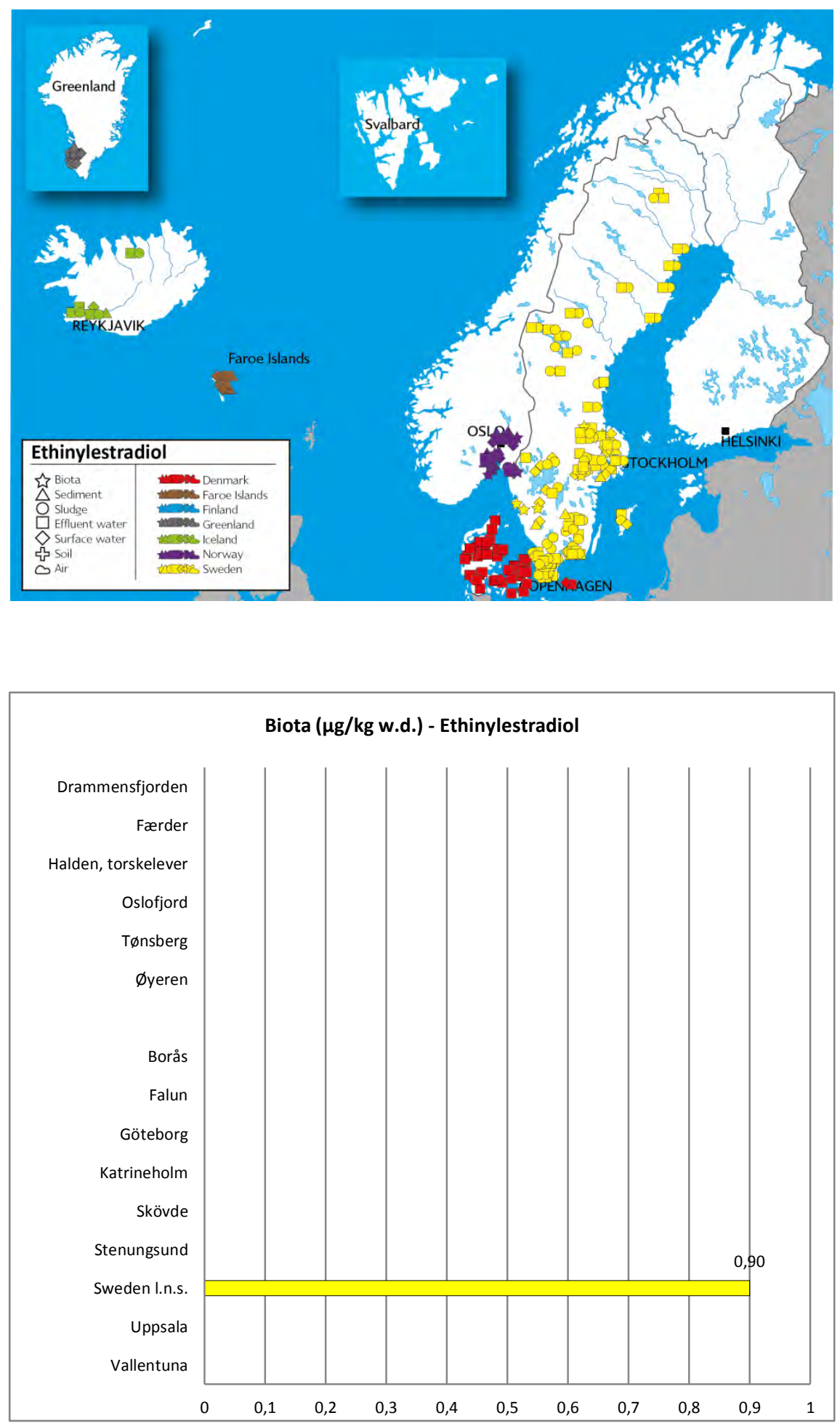


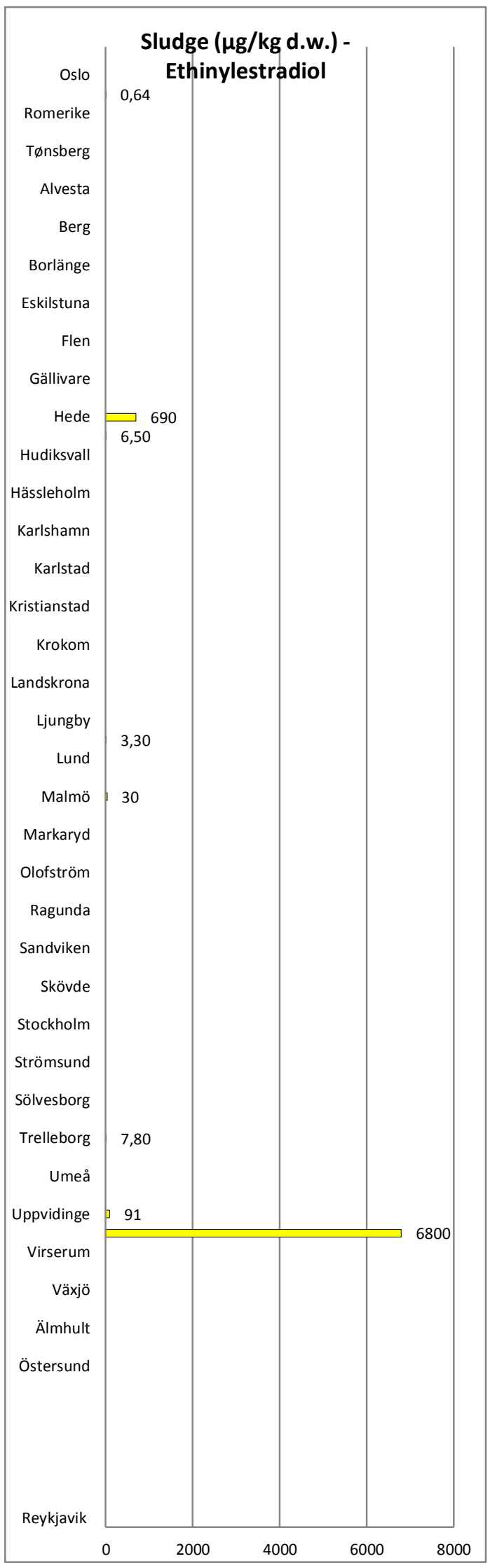




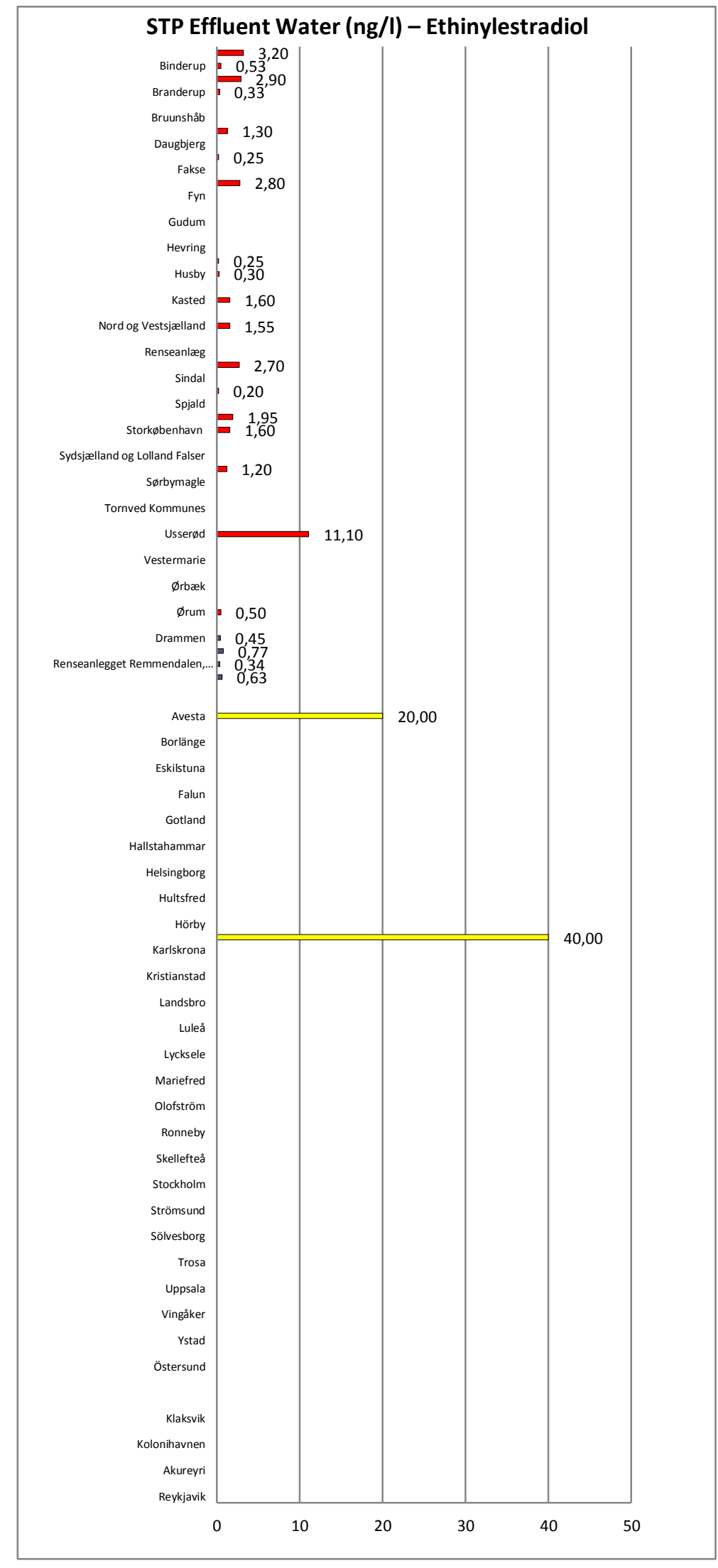




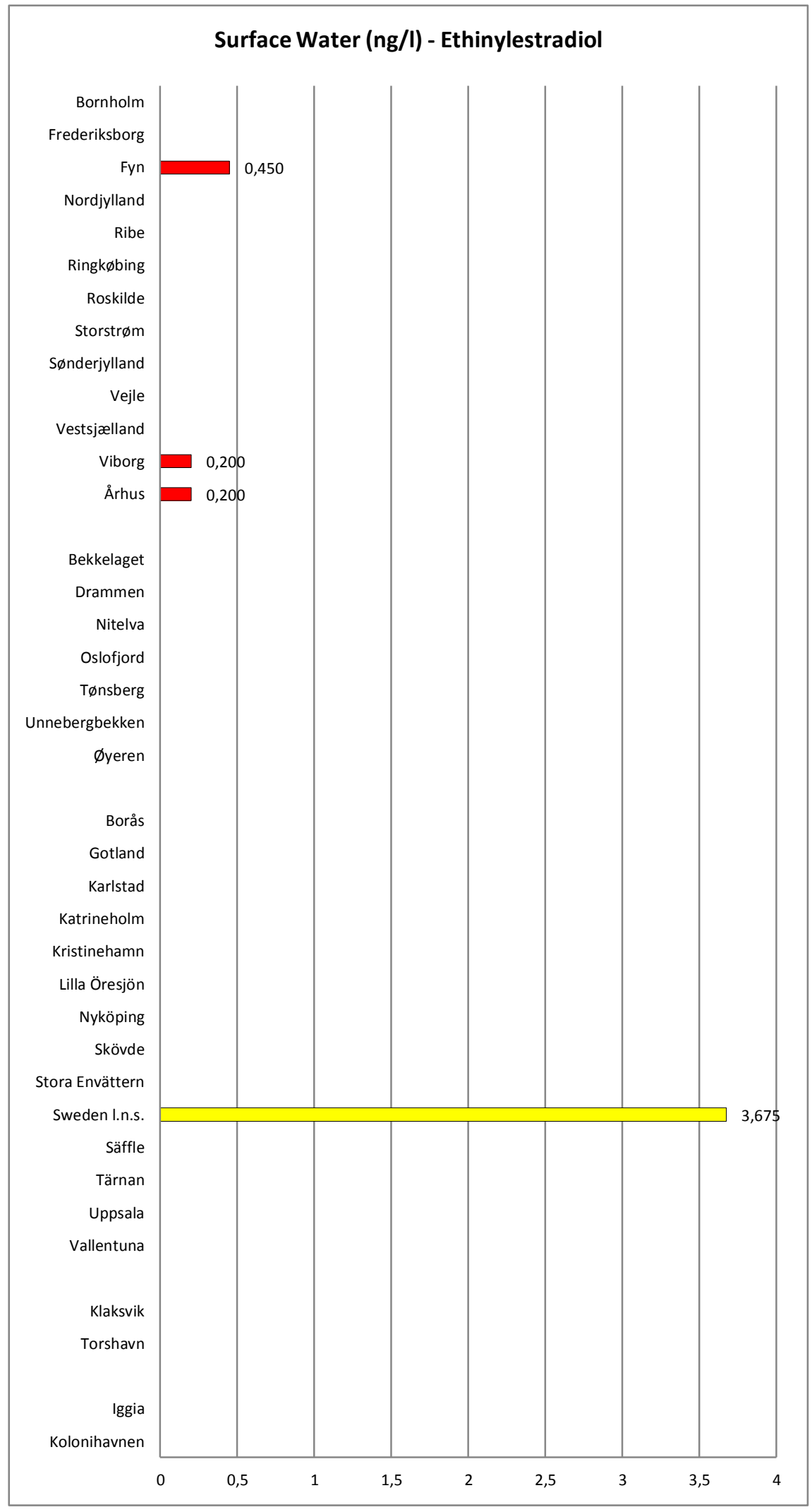




\begin{tabular}{|c|c|c|c|c|c|c|c|c|c|c|c|c|}
\hline $\begin{array}{l}\text { Ethinylestradiol } \\
\text { Matrix }\end{array}$ & \multicolumn{2}{|c|}{ Norway } & \multicolumn{2}{|c|}{ Denmark } & \multicolumn{2}{|c|}{ Sweden } & \multicolumn{2}{|c|}{ Faroe Islands } & \multicolumn{2}{|c|}{ Greenland } & \multicolumn{2}{|c|}{ Iceland } \\
\hline Matrix & $\begin{array}{r}\min \\
\max \\
A D\end{array}$ & $\begin{array}{l}\text { Nr.S } \\
\text { (ref) }\end{array}$ & $\begin{array}{r}\min \\
\max \\
A D\end{array}$ & $\begin{array}{l}\text { Nr.S } \\
\text { (ref) }\end{array}$ & $\begin{array}{r}\min \\
\max \\
A D\end{array}$ & $\begin{array}{l}\text { Nr.S } \\
\text { (ref) }\end{array}$ & $\begin{array}{r}\min \\
\max \\
A D\end{array}$ & $\begin{array}{l}\text { Nr.S } \\
\text { (ref) }\end{array}$ & $\begin{array}{r}\min \\
\max \\
A D\end{array}$ & $\begin{array}{l}\text { Nr.S } \\
\text { (ref) }\end{array}$ & $\begin{array}{r}\min \\
\max \\
A D\end{array}$ & $\begin{array}{l}\text { Nr.S } \\
\text { (ref) }\end{array}$ \\
\hline Biota $(\mu \mathrm{g} / \mathrm{kg} w \mathrm{w})$ & nd & 6 (a) & & & $\begin{array}{r}\text { nd } \\
0,90 \\
0,90\end{array}$ & $18(\mathrm{fg})$ & & & & & & \\
\hline $\begin{array}{l}\text { STP sludge }(\mu \mathrm{g} / \mathrm{kg} \\
\mathrm{dw})\end{array}$ & $\begin{array}{r}\text { nd } \\
0,64 \\
0,64\end{array}$ & $13(a b)$ & & & $\begin{array}{r}\text { nd } \\
6800 \\
1090\end{array}$ & 53 (f) & nd & $2(h)$ & nd & $2(h)$ & nd & $3(h)$ \\
\hline $\begin{array}{l}\text { Sediments }(\mu \mathrm{g} / \mathrm{kg} \\
\mathrm{dw})\end{array}$ & nd & 12 (a) & & & nd & 7 (f) & nd & $3(h)$ & nd & $2(h)$ & & \\
\hline $\begin{array}{l}\text { Landfild leachate } \\
\text { sediments }(\mu \mathrm{g} / \mathrm{kg} \\
\mathrm{dw})\end{array}$ & nd & 4 (a) & & & & & & & & & & \\
\hline $\begin{array}{l}\text { Untreated landfill } \\
\text { leachates, water } \\
\text { (ng/l) }\end{array}$ & $\begin{array}{r}\text { nd } \\
2,80 \\
2,80\end{array}$ & 4 (a) & & & $\begin{array}{l}\text { nd } \\
35 \\
30\end{array}$ & 7 (f) & & & & & & \\
\hline $\begin{array}{l}\text { Treated landfill } \\
\text { leachates, water } \\
\text { (ng/l) }\end{array}$ & $\begin{array}{r}\text { nd } \\
5 \\
5\end{array}$ & 5 (a) & & & & & & & & & & \\
\hline $\begin{array}{l}\text { Surface water } \\
\text { (ng/l) }\end{array}$ & nd & 8 (a) & $\begin{array}{r}\text { nd } \\
0,70 \\
0,30\end{array}$ & $\begin{array}{l}124 \\
\text { (ce) }\end{array}$ & $\begin{array}{r}\text { nd } \\
10 \\
3,69\end{array}$ & $34(\mathrm{fg})$ & nd & $5(h)$ & nd & $5(h)$ & & \\
\hline $\begin{array}{l}\text { STP Influent } \\
\text { (ng/l) }\end{array}$ & $\begin{array}{r}\text { nd } \\
1,70 \\
0,81\end{array}$ & $8(a)$ & $\begin{array}{r}\text { nd } \\
2,60 \\
1,49\end{array}$ & 11 (c) & $\begin{array}{l}\text { nd } \\
50 \\
50\end{array}$ & 74 (fg) & nd & $2(h)$ & & & nd & $5(h)$ \\
\hline $\begin{array}{l}\text { STP effluent } \\
\text { (ng/l) }\end{array}$ & $\begin{array}{r}\text { nd } \\
0,77 \\
0,55\end{array}$ & $11(a b)$ & $\begin{array}{r}\text { nd } \\
11,10 \\
1,53\end{array}$ & $\begin{array}{r}103 \\
\text { (cde) }\end{array}$ & $\begin{array}{l}\text { nd } \\
40 \\
40\end{array}$ & $73(\mathrm{fg})$ & nd & $1(\mathrm{~h})$ & nd & $1(\mathrm{~h})$ & nd & $5(h)$ \\
\hline $\begin{array}{l}\text { Drinking water } \\
\text { (ng/l) }\end{array}$ & & & & & & & & & & & & \\
\hline $\begin{array}{l}\text { Manure }(\mu \mathrm{g} / \mathrm{kg} \\
\mathrm{dw})\end{array}$ & & & & & $\begin{array}{l}\text { nd } \\
82 \\
82\end{array}$ & 5 (f) & & & & & & \\
\hline $\begin{array}{l}\text { Drinking water } \\
\text { (ng/l) }\end{array}$ & & & & & nd & 8 (g) & & & & & & \\
\hline $\begin{array}{l}\text { Hospital WW } \\
(\mathrm{ng} / \mathrm{l})\end{array}$ & & & & & $\begin{array}{l}\text { nd } \\
20 \\
15\end{array}$ & $7(f)$ & nd & $2(h)$ & nd & $2(h)$ & & \\
\hline $\begin{array}{l}\text { Miscellaneous }{ }^{69} \\
(\mathrm{ng} / \mathrm{l})\end{array}$ & & & $\begin{array}{r}\text { nd } \\
3,70 \\
2,57\end{array}$ & 107 (c) & & & & & & & & \\
\hline
\end{tabular}

\section{References:}

a) (Møskeland, et al., 2006)

b) (Thomas, et al., 2007)

c) (Stuer-Lauridsen, et al., 2005) 
d) (Kjølholt, et al., 2003)

e) (Ingerslev, et al., 2003)

f) (Andersson, et al., 2006)

g) (Fick, et al., 2011)

h) (Huber, et al., NA)

\section{Conclusion:}

Ethinylestradiol was monitored in two studies in Norway, three studies in Denmark, two studies in Sweden, and one study in Faroe Islands, Greenland and Iceland.

The compound were detected in several matrices:

- Biota in Sweden

- STP sludge in Norway and Sweden

- Surface water in Denmark and Sweden. The highest concentrations were detected in Sweden. All the detected values were above the PNEC

- STP effluent water in Norway, Denmark and Sweden. High variation amongst the three countries. The lowest values were detected in Norway. Highest values were detected in Sweden. All the detected values were above the PNEC

- STP influent water in Norway, Denmark and Sweden. The highest values were detected in Sweden and fill leachates in Norway

- Miscellaneous sample of water in Denmark

\subsubsection{Tamoxifen}

CAS nr.: 10540-29-1

Info: Used for the treatment of both early and advanced ER+ (estrogen receptor positive) breast cancer in pre- and post-menopausal women. Additionally, it is the most common hormone treatment for male breast cancer.

Toxicity data:

LOEC $5600 \mathrm{ng} / \mathrm{l}$ (Schlabach, et al., 2009)

$P N E C=0,49 \mu \mathrm{g} / \mathrm{L}$ (Stuer-Lauridsen, et al., 2011) 

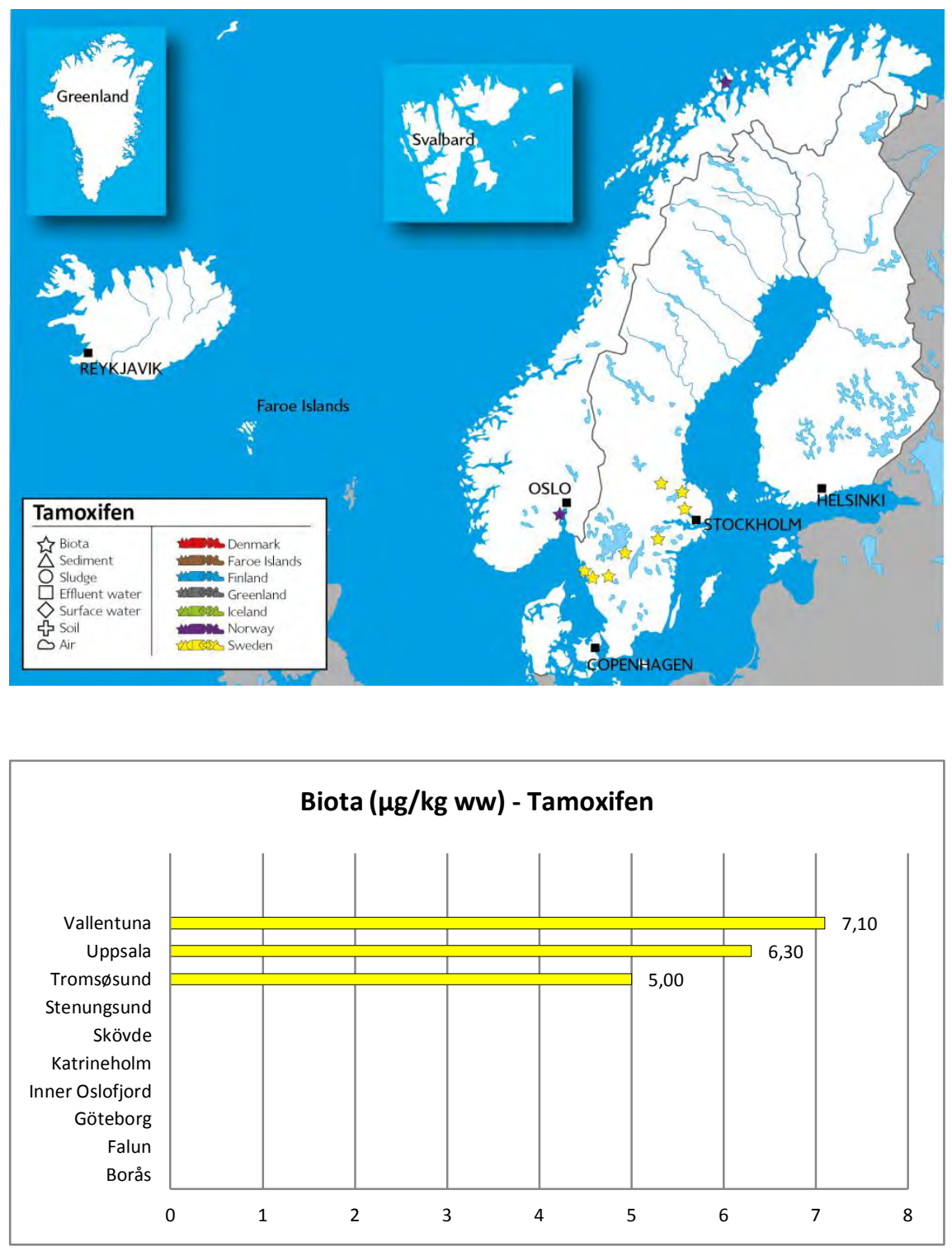

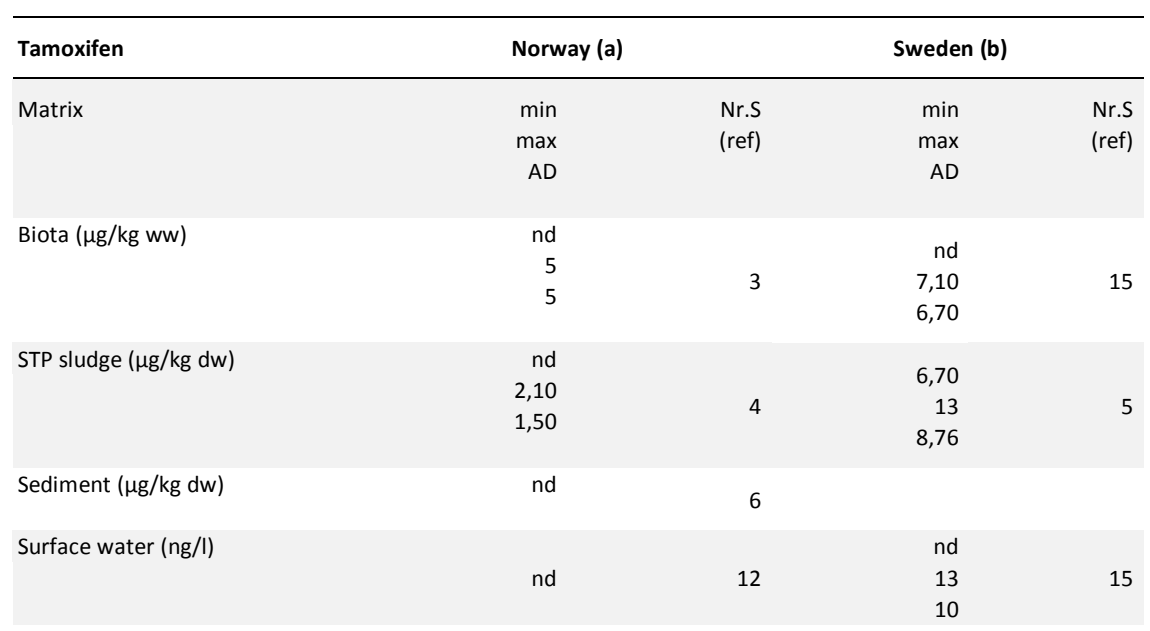




\begin{tabular}{lccr}
\hline Tamoxifen & Norway (a) & \multicolumn{2}{c}{ Sweden (b) } \\
\hline STP Influent (ng/l) & & nd & 12 \\
& & 930 & 208,96 \\
STP effluent (ng/l) & nd & nd & 13 \\
& & 210 & 6 \\
Drinking water (ng/l) & & 51,68 & nd \\
\hline
\end{tabular}

\section{References:}

a) (Schlabach, et al., 2009)

b) (Fick, et al., 2011)

\section{Conclusion}

The compound was monitored in one Norwegian and one Swedish study. It was detected in several matrices:

- Biota in both countries

- STP sludge both in Norway and, in highest consentrations, in Sweden

- STP effluent in Sweden

- Surface water in Sweden

- STP influent in Sweden

\subsection{Compounds monitored in one study}

\begin{tabular}{|c|c|c|c|}
\hline & Norethindrone (68-22-4) & Progesterone (57-83-0) & \\
\hline \multirow[t]{3}{*}{ Matrix } & $\min$ & $\min$ & Nr.S \\
\hline & $\max$ & $\max$ & \\
\hline & $A D$ & $A D$ & \\
\hline Biota & nd & nd & 3 \\
\hline \multirow[t]{3}{*}{ Sediment $(\mu \mathrm{g} / \mathrm{kg} \mathrm{dw})$} & nd & nd & 7 \\
\hline & 12,00 & 32 & \\
\hline & 12,00 & 32 & \\
\hline \multirow[t]{3}{*}{ STP sludge ( $\mu \mathrm{g} / \mathrm{kg} \mathrm{dw})$} & nd & nd & 61 \\
\hline & 6100 & 1900 & \\
\hline & 446,67 & 141,1 & \\
\hline \multirow[t]{3}{*}{ STP effluent (ng/l) } & nd & nd & 55 \\
\hline & 10 & 110 & \\
\hline & 9 & 29,26 & \\
\hline \multirow[t]{3}{*}{ Influent water $(\mu \mathrm{g} / \mathrm{l})$} & nd & nd & 19 \\
\hline & 20 & 22 & \\
\hline & 13,83 & 13,12 & \\
\hline \multirow[t]{3}{*}{ Leachate (ng/l) } & nd & nd & 7 \\
\hline & 40 & 20 & \\
\hline & 17,5 & 13,8 & \\
\hline \multirow[t]{3}{*}{ Surface water (ng/l) } & nd & nd & 17 \\
\hline & 10 & 32 & \\
\hline & 7,5 & 13,50 & \\
\hline
\end{tabular}




\begin{tabular}{lrrr}
\hline & Norethindrone (68-22-4) & Progesterone (57-83-0) & \\
\hline Hospital WW (ng/l) & nd & nd & 7 \\
& 30 & 20 & \\
& 23,5 & 11,25 & \\
Manure $(\mu \mathrm{g} / \mathrm{kg} \mathrm{dw})$ & nd & nd & 5 \\
& 66 & 200 & \\
& 54 & 112,7 & \\
\hline
\end{tabular}

\section{Reference:}

- (Andersson, et al., 2006)

\begin{tabular}{|c|c|c|c|c|c|}
\hline & $\begin{array}{l}\text { Etonogestrel } \\
(54048-10-1)\end{array}$ & $\begin{array}{r}\text { Finasteride } \\
(98319-26-7)\end{array}$ & $\begin{array}{r}\text { Flutamide } \\
(13311-84-7)\end{array}$ & $\begin{array}{r}\text { Levonorgestrel } \\
(797-63-7)\end{array}$ & \\
\hline \multirow[t]{3}{*}{ Matrix } & $\min$ & $\min$ & $\min$ & $\min$ & Nr.S \\
\hline & $\max$ & $\max$ & $\max$ & $\max$ & \\
\hline & $A D$ & $A D$ & $A D$ & $A D$ & \\
\hline Biota ( $\mu \mathrm{g} / \mathrm{kg})$ & nd & nd & nd & nd & 15 \\
\hline STP sludge $(\mu \mathrm{g} / \mathrm{kg} \mathrm{dw})$ & nd & nd & nd & nd & 5 \\
\hline \multirow[t]{3}{*}{ Effluent (ng/l) } & nd & nd & nd & nd & 13 \\
\hline & & 20 & 64 & & \\
\hline & & 16,6 & 49 & & \\
\hline \multirow[t]{3}{*}{ Surface water (ng/l) } & nd & nd & nd & nd & 15 \\
\hline & & 42 & & & \\
\hline & & 27 & & & \\
\hline \multirow[t]{3}{*}{ STP influent (ng/l) } & nd & nd & nd & nd & 12 \\
\hline & & 28 & 110 & & \\
\hline & & 17,6 & 48,3 & & \\
\hline Drinking water (ng/l) & nd & nd & nd & nd & 6 \\
\hline
\end{tabular}

\section{Reference:}

- (Fick, et al., 2011) 


\begin{tabular}{lcc}
\hline & 170-estradiol (57-91-0) & Nr.S \\
\hline Matrix & $\min$ & \\
& AD & 124 \\
Surface water $(\mathrm{ng} / \mathrm{l})$ & $\mathrm{nd}$ & \\
& 1,8 & 14 \\
& 0,71 & \\
Influent water $(\mu \mathrm{g} / \mathrm{l})$ & $\mathrm{nd}$ & 74 \\
& 19,5 & \\
& 5,36 & $106^{70}$ \\
STP effluent $(\mu \mathrm{g} / \mathrm{l})$ & $\mathrm{nd}$ & \\
& 51,9 & \\
\end{tabular}

\section{Reference:}

- (Stuer-Lauridsen, et al., 2005) 


\section{Cardiac and blood pressure medicine and diuretics}

\subsection{Scope and definition}

Cardiac medication includes drugs which treat heart diseases.

Blood pressure medicine is defined as drugs that aim to treat hyperor hypotension.

Cardiac and blood pressure medication includes beta-blockers, ACE inhibitors, angiotensin II receptor blockers, calcium channel blockers, alpha blockers, alpha- 2 receptor agonist, combined alpha and betablockers, central agonists, peripheral adrenergic inhibitors, blood vessel dilators, or vasodilators.

Diuretics increase the amount of sodium and water excreted into the urine by the kidneys, one effect is to lower blood pressure mainly by reducing the volume of fluid in the blood vessels. In this manner, there is a large overlap between diuretics and blood pressure medicine, as most drugs classified as "diuretics" are often also used in the treatment of hypertension. Consequently, diuretics are included in this category.

Antithrombotics were not include in this category.

Table 4 presents compounds belonging to this category and identified in monitoring studies in the Nordic countries.

Table 4: Cardiac, blood pressure and diuretics identified in monitoring studies in the Nordic countries

\begin{tabular}{ll}
\hline Compounds Monitored & CAS nr \\
\hline Acebutolol (betablocker) & $37517-30-9$ \\
Alfuzosin (alfa blocker, diuretic) & $81403-80-7$ \\
Amiloride (diuretic, used for hypertension) & $2016-88-8$ \\
Amlodipin (used for hypertension) & $88150-42-9$ \\
Atenolol (betablocker) & $29122-68-7$ \\
Bendroflumethiazid (used for hypertension) & $73-48-3$ \\
Bisoprolol (betablocker) & $66722-44-9$ \\
Candesartan (angiotensin Il receptor antagonist) & $139481-59-7$ \\
Cilazapril (ACE inhibitor) & $88768-40-5$ \\
Diltiazem (anti-hypertension) & $42399-41-7$ \\
Enalapril (angiotensin converting enzyme (ACE inhibitor) & $75847-73-3$ \\
Eprosartan (used for hypertension) & $133040-01-4$ \\
Felodipine (used for hypertension) & $72509-76-3$ \\
Furosemide(diuretic, used for hypertension) & $54-31-9$ \\
Hydrochlorothiazide (diuretic used for hypertension and congestive heart faillure) & $58-93-5$ \\
Irbesartan (anti-hypertension) & $138402-11-6$ \\
Losartan & $124750-99-8$ \\
Metoprolol (betablocker) & $51384-51-1$ \\
Propanolol (Beta blocker) & $525-66-6$ \\
Sotalol (betablocker) & $3930-20-9$ \\
Telmisartan (used for hypertension) & $144701-48-4$ \\
Verapamil (calcium channel blocker ) & $52-53-9$ \\
\hline
\end{tabular}




\subsection{Compounds mapped}

\subsubsection{Amlodipine}

CAS nr.: 88150-42-9

Info: Cardioselective beta blocker with ISA (intrinsic sympathomimetic) activity. Long-acting calcium channel blocker (dihydropyridine (DHP) class) used as an anti-hypertensive and in the treatment of angina.

Toxicity data: NA

$P N E C=\mathrm{NA}$

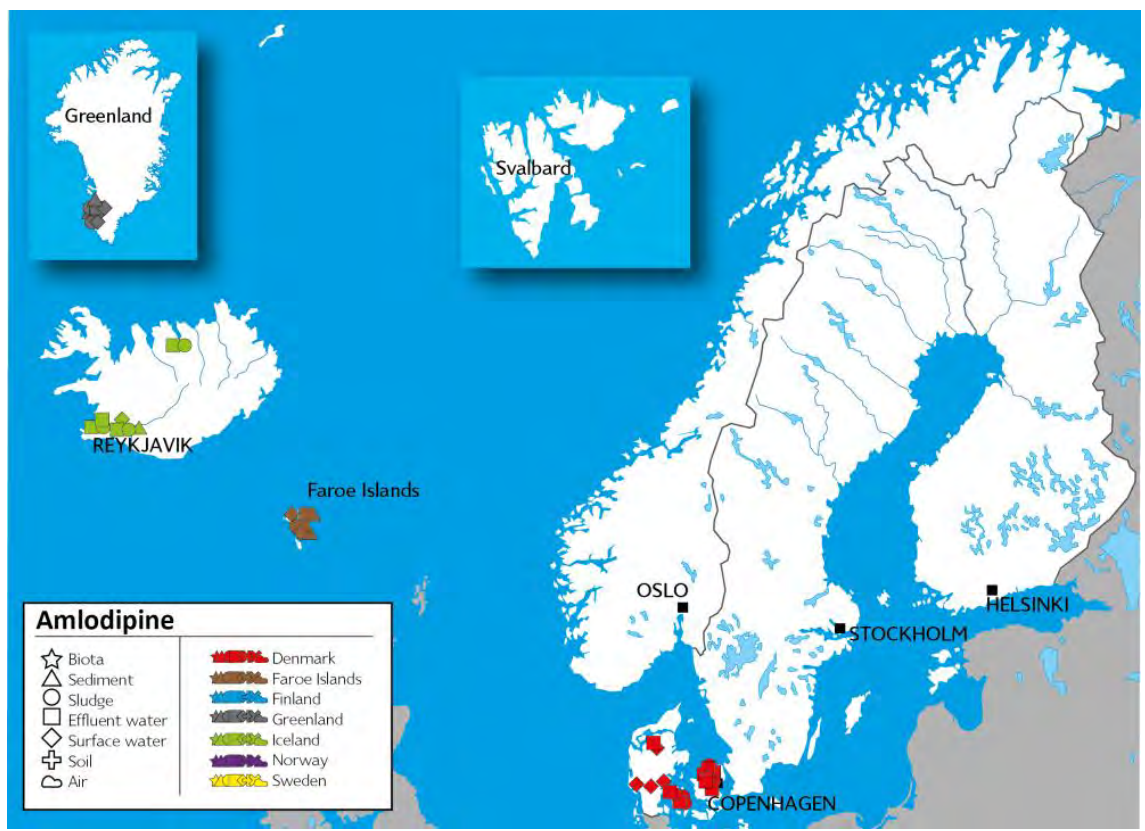



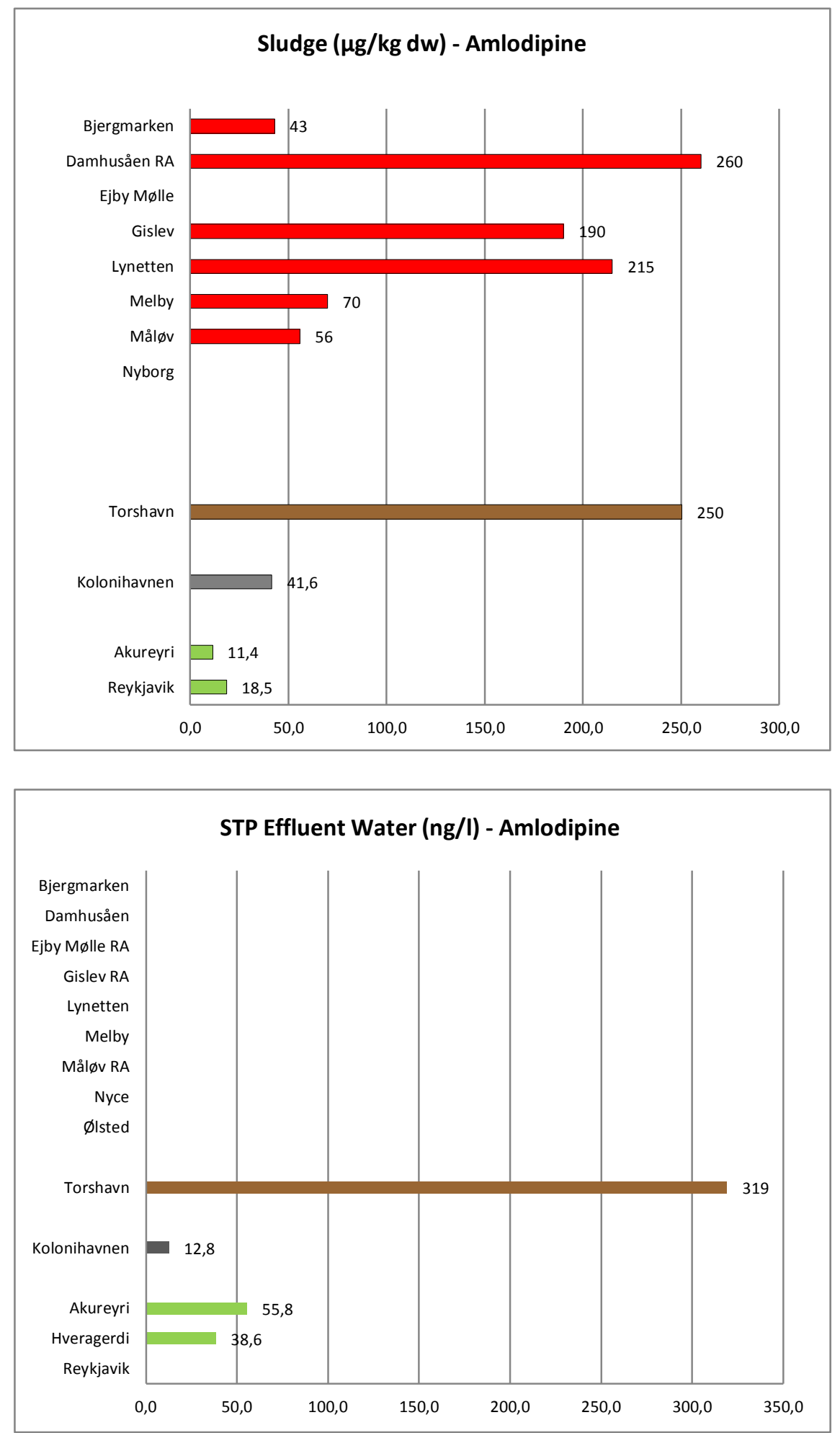


\begin{tabular}{|c|c|c|c|c|c|c|c|c|}
\hline $\begin{array}{l}\text { Amlodipine } \\
\text { Matrix }\end{array}$ & \multicolumn{2}{|c|}{ Denmark } & \multicolumn{2}{|c|}{ Faroe Islands } & \multicolumn{2}{|c|}{ Greenland } & \multicolumn{2}{|c|}{ Iceland } \\
\hline & $\min$ & $\begin{array}{l}\text { Nr.S } \\
\text { (ref) }\end{array}$ & $\min$ & $\begin{array}{l}\text { Nr.S } \\
\text { (ref) }\end{array}$ & $\min$ & $\begin{array}{l}\text { Nr.S } \\
\text { (ref) }\end{array}$ & $\min$ & $\begin{array}{l}\text { Nr.S } \\
\text { (ref) }\end{array}$ \\
\hline \multicolumn{9}{|l|}{ Biota $(\mu \mathrm{g} / \mathrm{kg} w w)$} \\
\hline STP sludge $(\mu \mathrm{g} / \mathrm{kg} \mathrm{dw})$ & $\begin{array}{r}\text { nd } \\
310 \\
163,63\end{array}$ & 10 (a) & $\begin{array}{l}214 \\
286 \\
250\end{array}$ & 2 (b) & $\begin{array}{l}37,5 \\
45,7 \\
41,6\end{array}$ & 2 (b) & $\begin{array}{r}9,6 \\
18,5 \\
13,7\end{array}$ & 3 (b) \\
\hline Sediment $(\mu \mathrm{g} / \mathrm{kg} \mathrm{dw})$ & & & nd & $3(b)$ & $\begin{array}{r}\text { nd } \\
1,47 \\
1,47\end{array}$ & 2 (b) & & \\
\hline Surface water (ng/l) & nd & $18^{71}(\mathrm{a})$ & nd & 5 (b) & nd & 5 (b) & & \\
\hline Groundwater (ng/l) & nd & $3^{72}$ & & & & & & \\
\hline STP Influent (ng/l) & nd & 10 (a) & $\begin{array}{r}\text { nd } \\
247 \\
247\end{array}$ & 2 (b) & & & $\begin{array}{r}\text { nd } \\
11,4 \\
11,4\end{array}$ & 5 (b) \\
\hline STP effluent (ng/l) & nd & $10(a)$ & $\begin{array}{l}319 \\
319 \\
319\end{array}$ & 1 (b) & $\begin{array}{l}12,8 \\
12,8 \\
12,8\end{array}$ & 1 (b) & $\begin{array}{r}\text { nd } \\
72,9 \\
47,2\end{array}$ & 5 (b) \\
\hline Hospital WW (ng/I) & & & $\begin{array}{r}98,1 \\
448 \\
273\end{array}$ & 2 (b) & $\begin{array}{r}\text { nd } \\
121 \\
121\end{array}$ & 2 (b) & & \\
\hline Pig slurry (ng/l) & nd & 4 (a) & & & & & & \\
\hline $\begin{array}{l}\text { Warning system for pesticide } \\
\text { leaching to groundwater (ng/l) }\end{array}$ & nd & 9 (a) & & & & & & \\
\hline
\end{tabular}

\section{References:}

a) (Mogensen, et al., 2008)

b) (Huber, et al., NA)

\section{Conclusion:}

The compound was monitored in Denmark, Faroe Islands, Greenland and Iceland. It was detected in several matrices:

- STP sludge in all countries, in most samples analysed. Values detected in Denmark and the Faroe Island were significantly higher than in the other countries

- In only one sediment sample in Greenland

- Not detected in surface water in any country monitored

- STP effluent in Faroe Islands, Greenland and Iceland but not Denmark. High concentrations were detected in Faroe Islands

- Hospital waste water in Faroe Islands, in one sample in Greenland

- STP influent in Faroe Islands and Iceland, not in Denmark

71 Upstream and downstream of fish farms

72 Including 2 measurements in groundwater at proximity of a hospital 


\subsubsection{Atenolol}

CAS nr.: 29122-68-7

Info: Selective beta-1 receptor antagonist

Toxicity data: NA

$P N E C=77,7 \mu \mathrm{g} / \mathrm{l}$
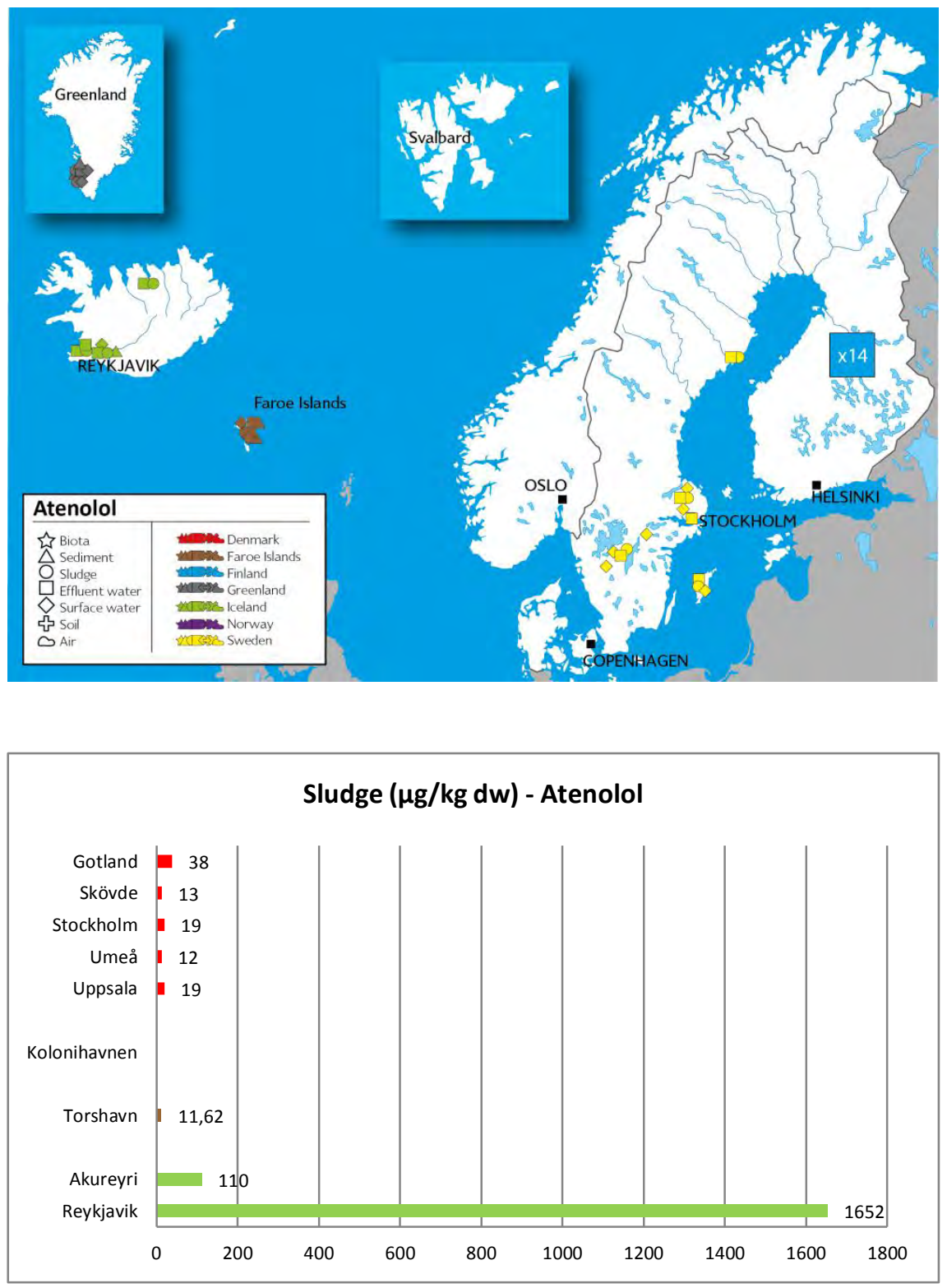

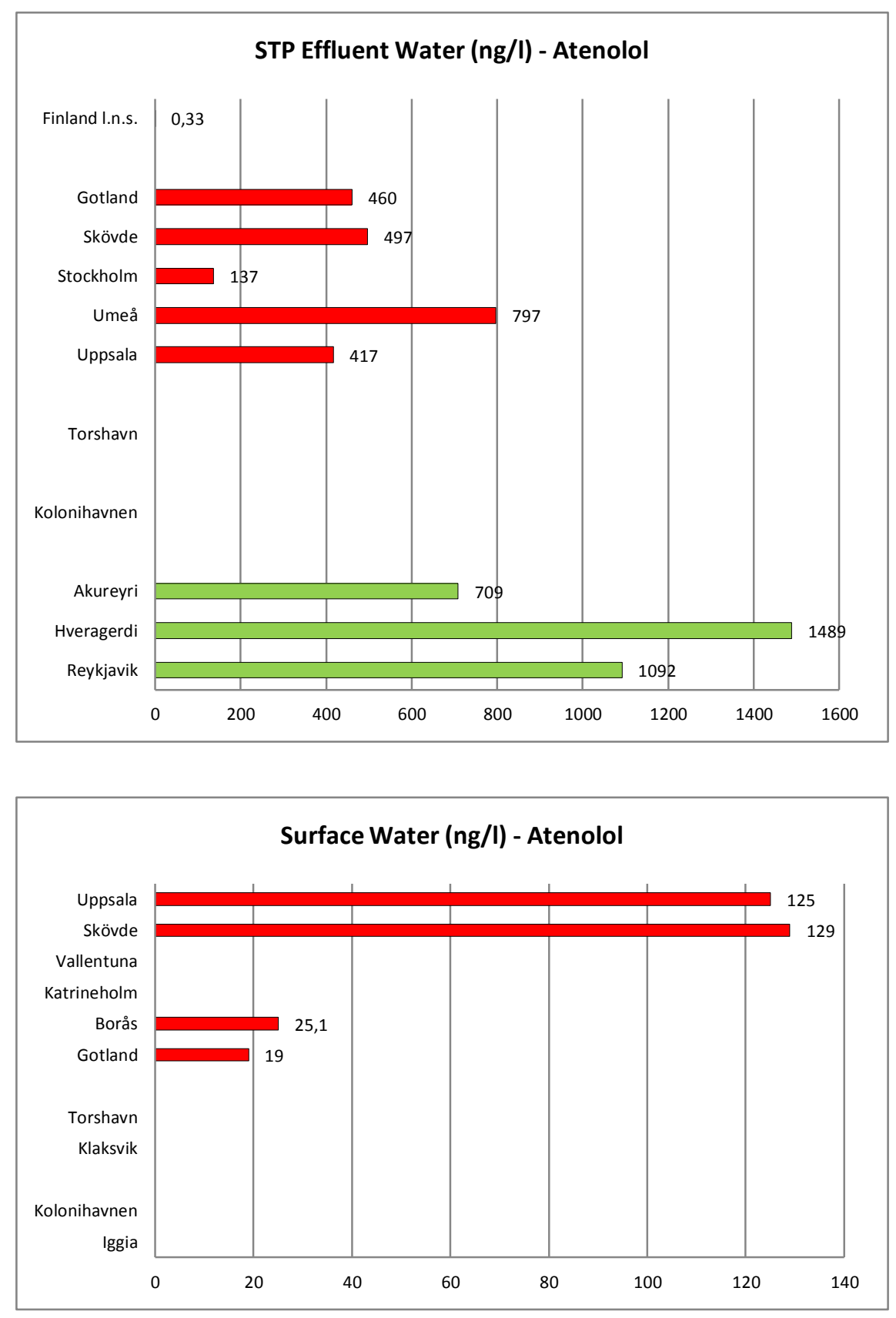

\begin{tabular}{|c|c|c|c|c|c|c|c|c|c|c|}
\hline $\begin{array}{l}\text { Atenolol } \\
\text { Matrix }\end{array}$ & \multicolumn{2}{|c|}{ Finland } & \multicolumn{2}{|c|}{ Sweden } & \multicolumn{2}{|c|}{ Faroe Islands } & \multicolumn{2}{|c|}{ Greenland } & \multicolumn{2}{|c|}{ Iceland } \\
\hline Matrix & $\begin{array}{r}\min \\
\max \\
A D\end{array}$ & $\begin{array}{l}\text { Nr.S } \\
\text { (ref) }\end{array}$ & $\begin{array}{r}\min \\
\max \\
A D\end{array}$ & $\begin{array}{l}\text { Nr.S } \\
\text { (ref) }\end{array}$ & $\begin{array}{r}\min \\
\max \\
A D\end{array}$ & $\begin{array}{l}\text { Nr.S } \\
\text { (ref) }\end{array}$ & $\begin{array}{r}\min \\
\max \\
A D\end{array}$ & $\begin{array}{l}\text { Nr.S } \\
\text { (ref) }\end{array}$ & $\begin{array}{r}\min \\
\max \\
A D\end{array}$ & $\begin{array}{l}\text { Nr.S } \\
\text { (ref) }\end{array}$ \\
\hline $\begin{array}{l}\text { Biota }(\mu \mathrm{g} / \mathrm{kg} \\
\text { ww) }\end{array}$ & & & nd & 15 (c) & & & & & & \\
\hline $\begin{array}{l}\text { STP sludge } \\
(\mu \mathrm{g} / \mathrm{kg} \mathrm{dw})\end{array}$ & & & $\begin{array}{r}12 \\
38 \\
20,2\end{array}$ & 5 (c) & $\begin{array}{r}9,82 \\
13,4 \\
11,62\end{array}$ & 2 (d) & nd & 2 (d) & $\begin{array}{r}58,6 \\
1651,6 \\
623,8\end{array}$ & 3 (d) \\
\hline $\begin{array}{l}\text { Sediment } \\
(\mu \mathrm{g} / \mathrm{kg} \mathrm{dw})\end{array}$ & & & & & nd & $3(d)$ & nd & 2 (d) & & \\
\hline
\end{tabular}




\begin{tabular}{|c|c|c|c|c|c|c|c|c|c|c|}
\hline Atenolol & \multicolumn{2}{|c|}{ Finland } & \multicolumn{2}{|c|}{ Sweden } & \multicolumn{2}{|c|}{ Faroe Islands } & \multicolumn{2}{|c|}{ Greenland } & \multicolumn{2}{|c|}{ Iceland } \\
\hline (ng/l) & & & $\begin{array}{r}390 \\
74\end{array}$ & 15 (c) & nd & 5 (d) & nd & 5 (d) & & \\
\hline \multicolumn{11}{|l|}{$\begin{array}{l}\text { Groundwater } \\
(\mu \mathrm{g} / \mathrm{l})\end{array}$} \\
\hline $\begin{array}{l}\text { STP Influent } \\
\text { (ng/l) }\end{array}$ & $\begin{array}{l}\text { NA } \\
\text { NA } \\
800\end{array}$ & 11 (a) & $\begin{array}{r}330 \\
4900 \\
1160\end{array}$ & 12 (c) & $\begin{array}{l}\text { nd } \\
36 \\
36\end{array}$ & 2 (d) & & & $\begin{array}{r}500 \\
2230 \\
1120\end{array}$ & 5 (d) \\
\hline $\begin{array}{l}\text { STP effluent } \\
\text { (ng/l) }\end{array}$ & $\begin{array}{l}\text { NA } \\
\text { NA } \\
330\end{array}$ & $14(a)$ & $\begin{array}{l}130 \\
920 \\
460\end{array}$ & 13 (c) & nd & 1 (d) & nd & 1 (d) & $\begin{array}{r}706,9 \\
1729,2 \\
1097,4\end{array}$ & 5 (d) \\
\hline $\begin{array}{l}\text { Hospital WW } \\
(\mathrm{ng} / \mathrm{l})\end{array}$ & & & & & $\begin{array}{r}\text { nd } \\
120 \\
120\end{array}$ & 2 (d) & $\begin{array}{l}310 \\
470 \\
390\end{array}$ & 2 (d) & & \\
\hline Drinking water & & & nd & 6 (c) & & & & & & \\
\hline $\begin{array}{l}\text { Settleable } \\
\text { particulate } \\
\text { material }\end{array}$ & nd & 11 (b) & & & & & & & & \\
\hline
\end{tabular}

\section{References:}
a) (Vieno, 2008)
b) (Lahti \& Oikari, 2011)
c) (Fick, et al., 2011)
d) (Huber, et al., NA)

\section{Conclusion:}

The compound was monitored in two studies in Finland, one study in Sweden and one study throughout Faroe Islands, Greenland and Iceland. The compound was detected in several matrices:

- STP sludge in Sweden, Faroe Islands and Iceland, not in Greenland. Values detected in Iceland were much higher than in the other countries

- Surface water only in Sweden

- STP effluent in Finland, Sweden and Iceland, not in Faroe Islands and Greenland

- Hospital waste water in all countries monitored.

- STP influent in all countries monitored 


\subsubsection{Bendroflumethiazide}

CAS nr.: 73-48-3

Info: Diuretic, also used in the treatment of hypertension

Toxicity data: NA

$P N E C=\mathrm{NA}$
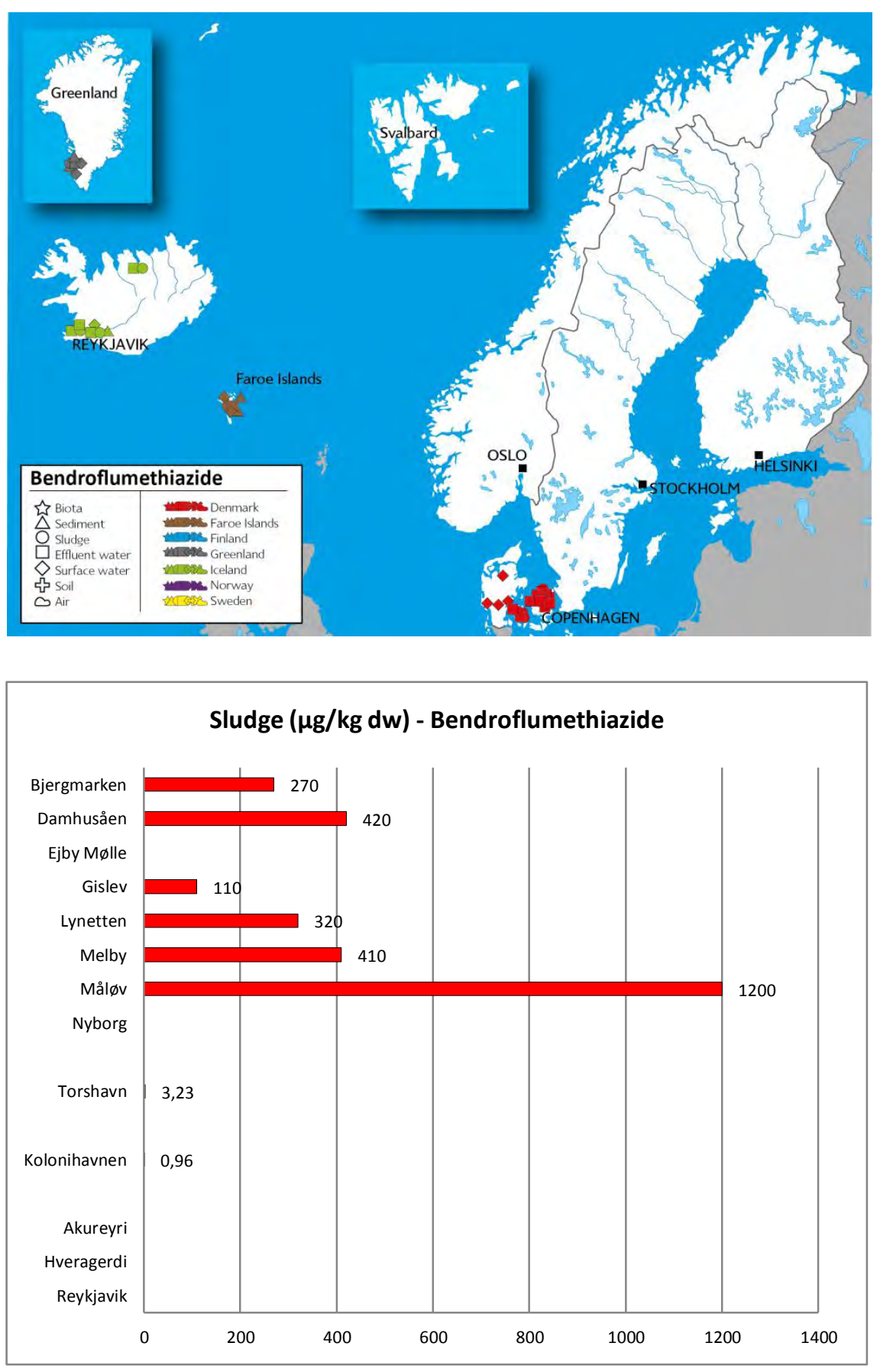

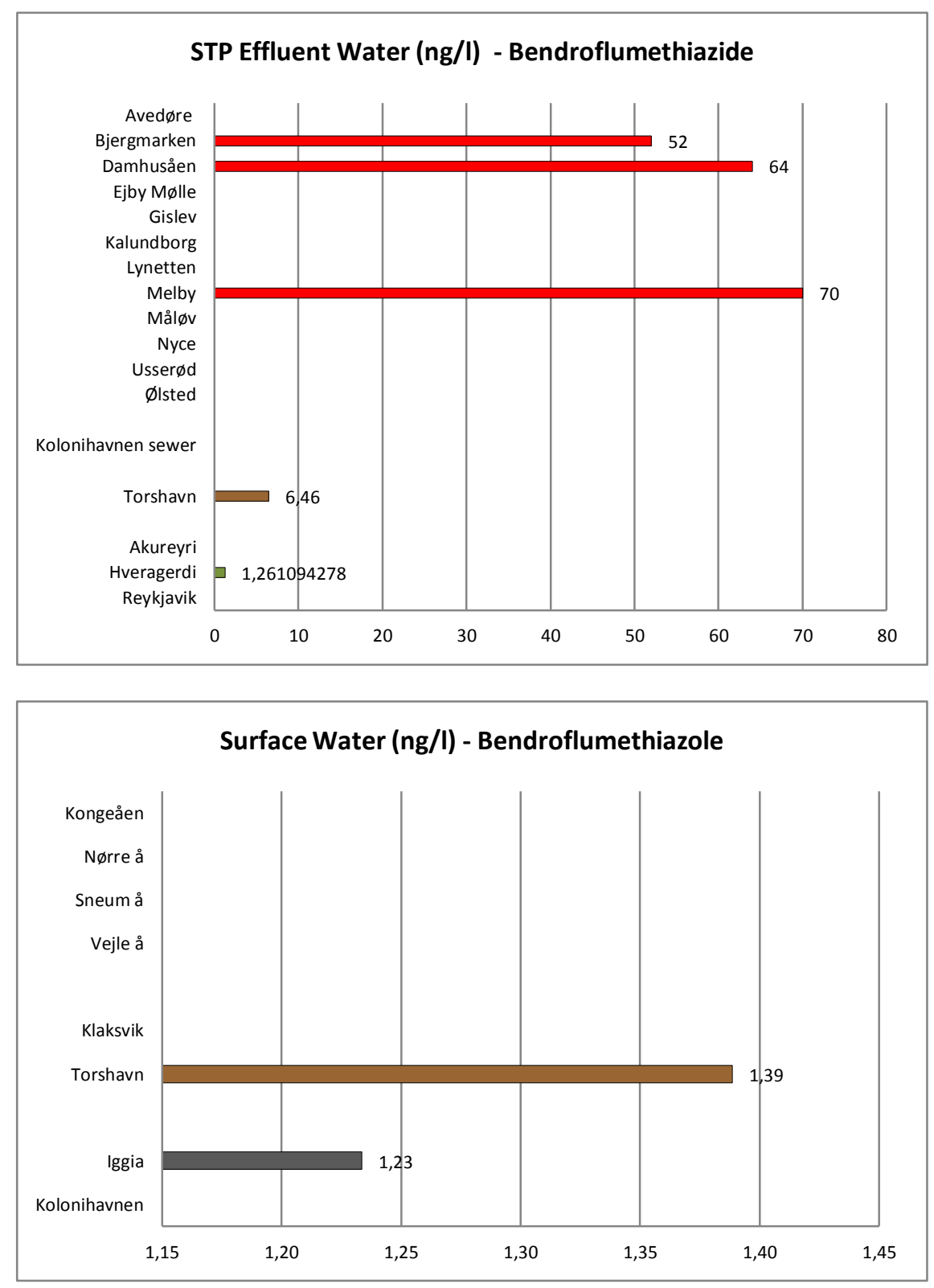

\begin{tabular}{|c|c|c|c|c|c|c|c|c|}
\hline \multirow{2}{*}{$\begin{array}{l}\text { Bendroflumethiazide } \\
\text { Matrix }\end{array}$} & \multicolumn{2}{|c|}{ Denmark } & \multicolumn{2}{|c|}{ Faroe Islands } & \multicolumn{2}{|c|}{ Greenland } & \multicolumn{2}{|c|}{ Iceland } \\
\hline & $\min$ & Nr.S & $\min$ & Nr.S & $\min$ & Nr.S & $\min$ & Nr.S \\
\hline & $\max$ & (ref) & $\max$ & (ref) & $\max$ & (ref) & $\max$ & (ref) \\
\hline & $A D$ & & $A D$ & & $A D$ & & $A D$ & \\
\hline \multirow[t]{3}{*}{ STP sludge $(\mu \mathrm{g} / \mathrm{Kg} \mathrm{dw})$} & nd & & nd & & 0,96 & & nd & \\
\hline & 1200 & $10(a)$ & 3,23 & 2 (c) & 0,97 & 2 (c) & nd & 4 (c) \\
\hline & 430 & & 3,23 & & 0,965 & & nd & \\
\hline
\end{tabular}




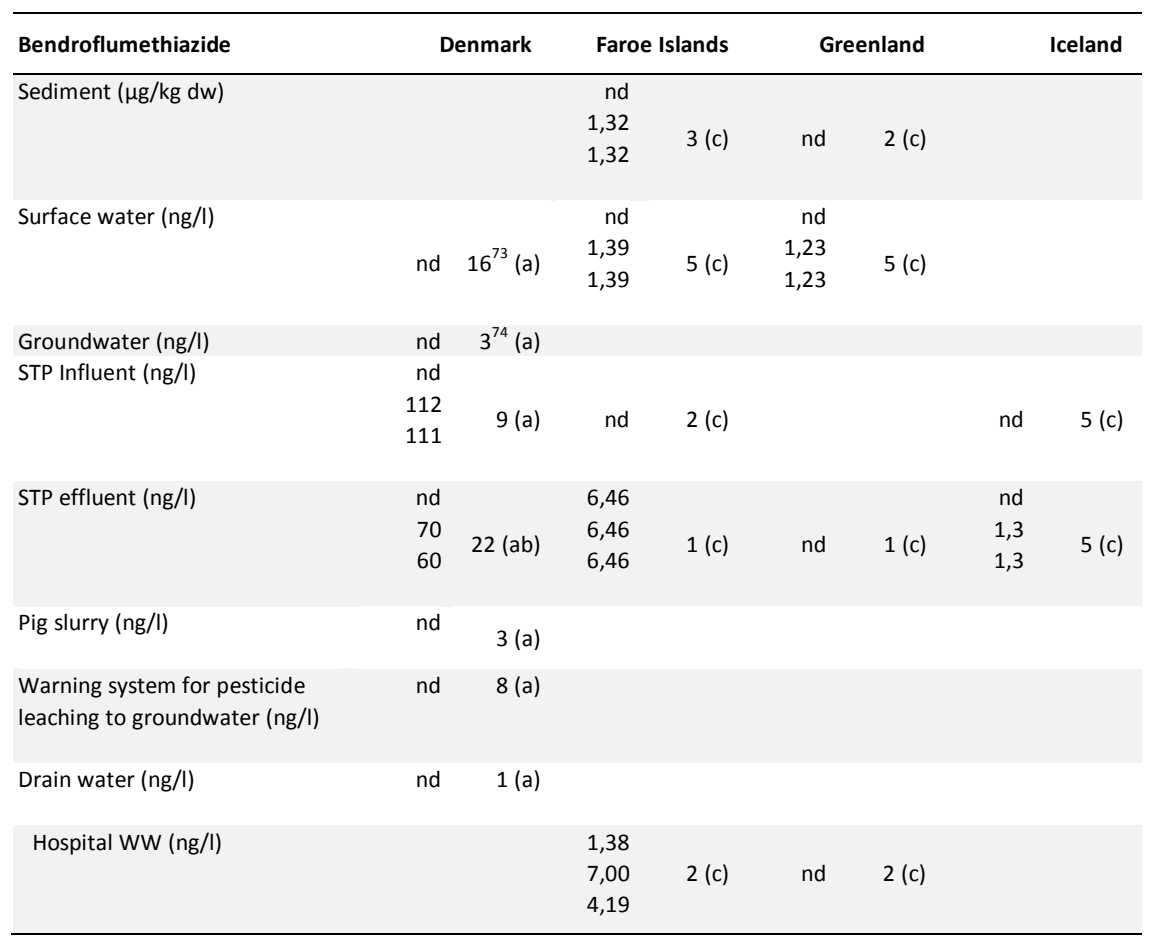

\section{References:}

a) (Mogensen, et al., 2008)

b) (Kjølholt, et al., 2003)

c) (Huber, et al., NA)

\section{Conclusion:}

The compound was monitored in Denmark, Faroe Islands, Greenland and Iceland. It was detected in several matrices:

- STP sludge in Denmark, Faroe Island and Greenland, not in Iceland. The value detected in one location in Denmark was much higher than all other values detected

- In one sample in sediment in Faroe islands

- Surface water in Faroe Islands and in Greenland

- STP effluent water in Denmark and Faroe Island, not in the other countries. The values detected in Denmark were higher

- Hospital waste water only in Faroe Islands

- STP influent only in Denmark

73 Upstream and downstream of fish farms

${ }^{74}$ Including 2 measurements in groundwater at proximity of a hospital 


\subsubsection{Furosemide}

CAS nr.: 54-31-9

Info: Diuretic, also used in the treatment of hypertension

Toxicity data: NA

$P N E C=45,14 \mu \mathrm{g} / \mathrm{l}$ (Stuer-Lauridsen, et al., 2011)

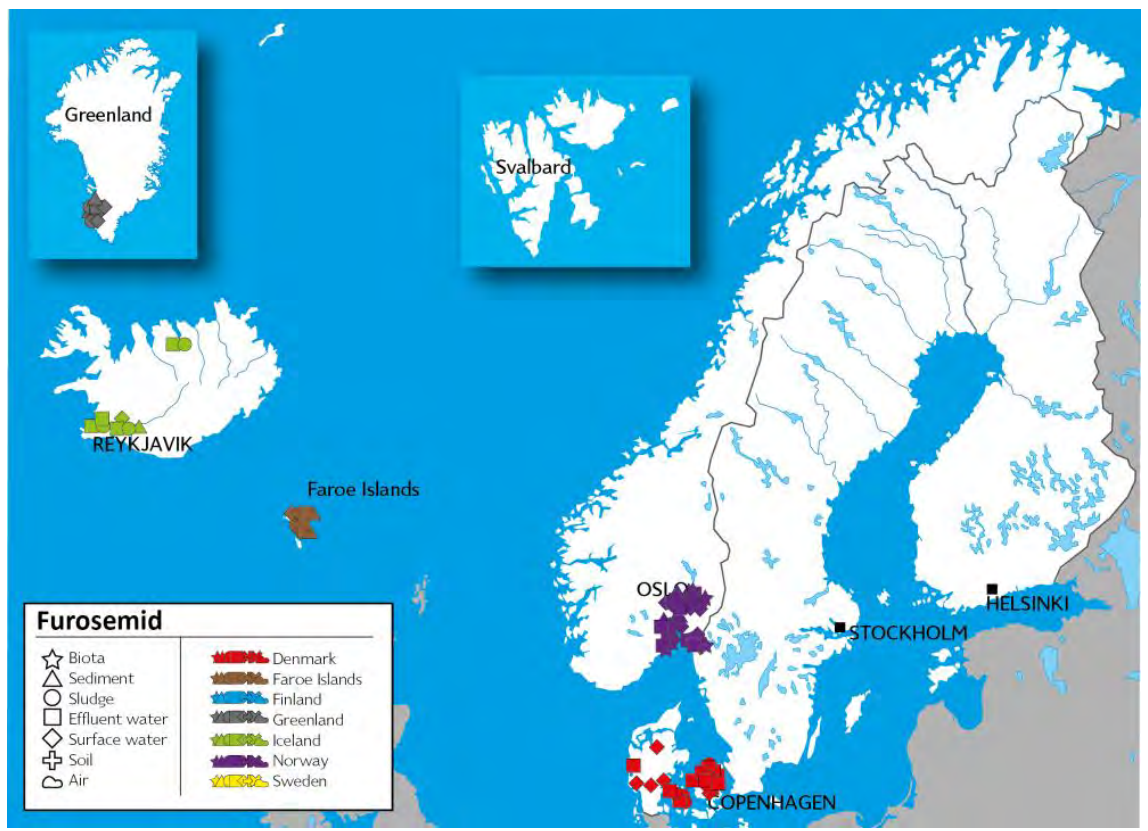




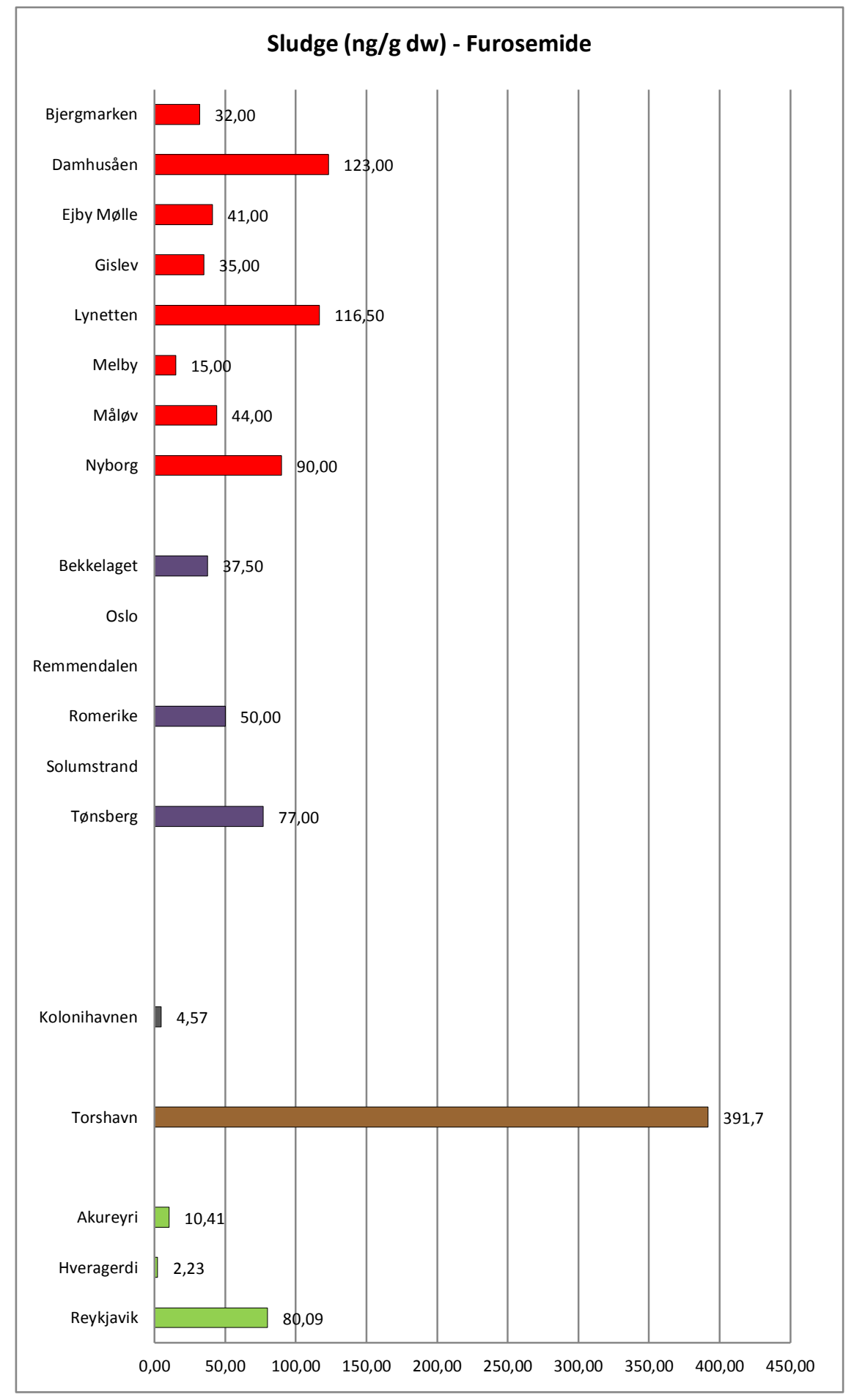




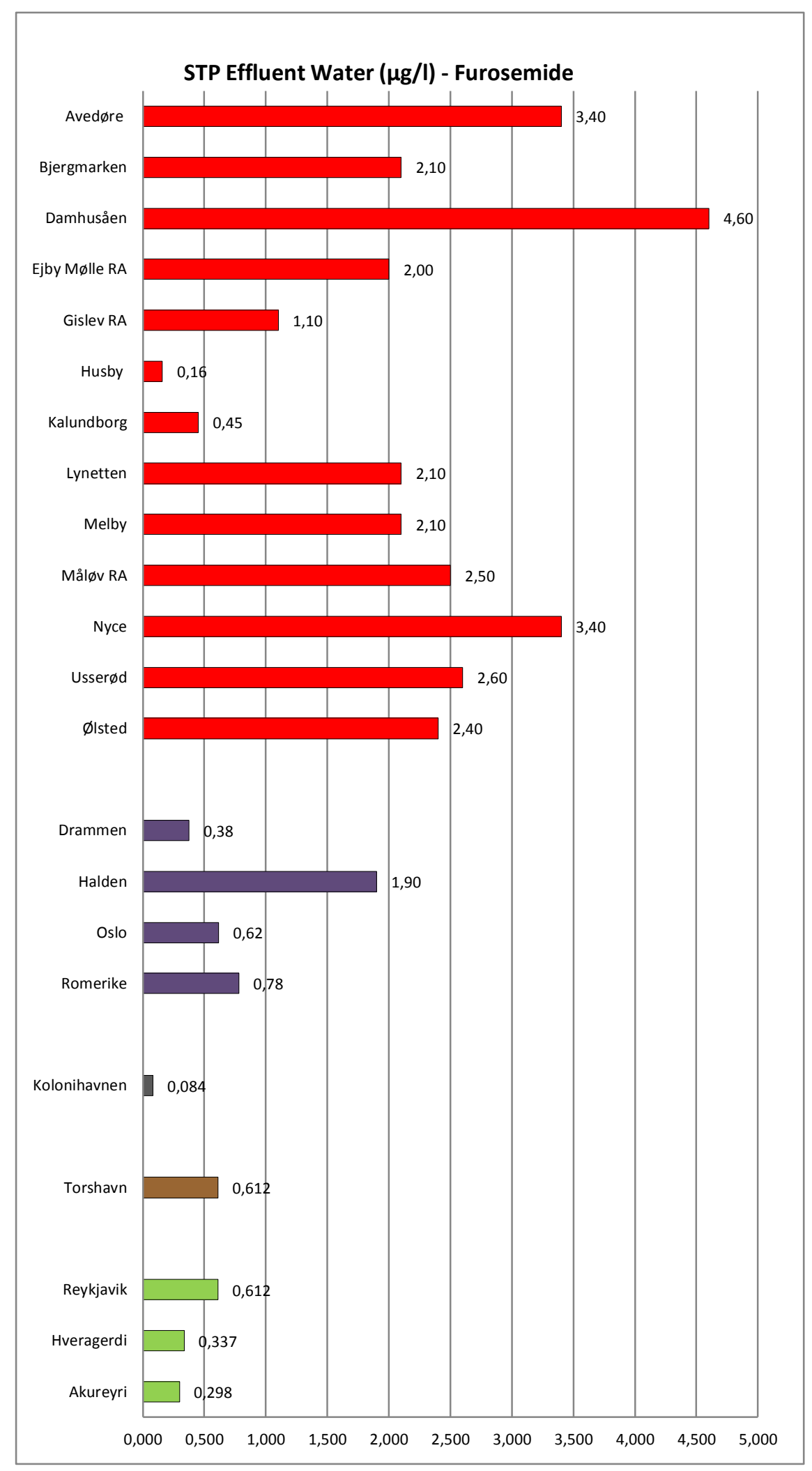




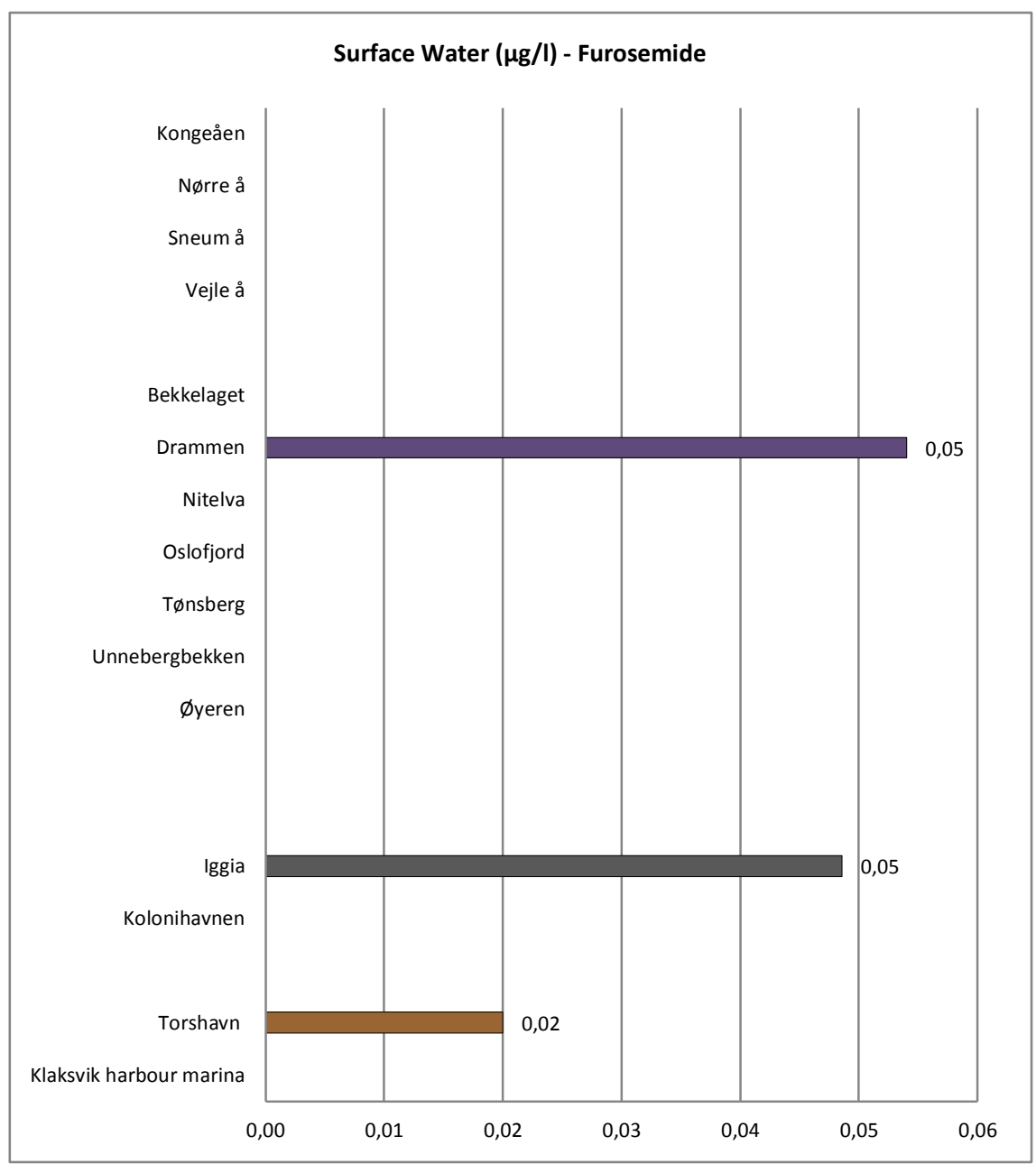

\begin{tabular}{|c|c|c|c|c|c|c|c|c|c|c|}
\hline $\begin{array}{l}\text { Furosemide } \\
\text { Matrix }\end{array}$ & \multicolumn{2}{|c|}{ Denmark } & \multicolumn{2}{|c|}{ Norway } & \multicolumn{2}{|c|}{ Faroe Islands } & \multicolumn{2}{|c|}{ Greenland } & \multicolumn{2}{|c|}{ Iceland } \\
\hline Matrix & $\begin{array}{r}\min \\
\max \\
A D\end{array}$ & $\begin{array}{l}\text { Nr.S } \\
\text { (ref) }\end{array}$ & $\begin{array}{r}\min \\
\max \\
A D\end{array}$ & $\begin{array}{l}\text { Nr.S } \\
\text { (ref) }\end{array}$ & $\begin{array}{r}\min \\
\max \\
A D\end{array}$ & $\begin{array}{l}\text { Nr.S } \\
\text { (ref) }\end{array}$ & $\begin{array}{r}\min \\
\max \\
A D\end{array}$ & $\begin{array}{l}\text { Nr.S } \\
\text { (ref) }\end{array}$ & $\begin{array}{r}\min \\
\max \\
A D\end{array}$ & $\begin{array}{l}\text { Nr.S } \\
\text { (ref) }\end{array}$ \\
\hline Biota $(\mu \mathrm{g} / \mathrm{kg} \mathrm{ww})$ & & & nd & $6(c)$ & & & & & & \\
\hline STP sludge $(\mu \mathrm{g} / \mathrm{kg} \mathrm{dw})$ & $\begin{array}{r}15 \\
180 \\
73,6\end{array}$ & $10(a)$ & $\begin{array}{r}\text { nd } \\
77 \\
54,8\end{array}$ & 6 (c) & $\begin{array}{r}97,7 \\
686 \\
391,7\end{array}$ & 2 (d) & $\begin{array}{l}2,96 \\
6,18 \\
4,57\end{array}$ & 2 (d) & $\begin{array}{r}2,2 \\
80,1 \\
25,8\end{array}$ & 4 (d) \\
\hline $\begin{array}{l}\text { Sediment }(\mu \mathrm{g} / \mathrm{kg} \mathrm{dw}) \\
\text { Landfild leachates } \\
\text { sediments ( } \mu \mathrm{g} / \mathrm{kg} \mathrm{dw})\end{array}$ & & & $\begin{array}{l}\text { nd } \\
\text { nd }\end{array}$ & $\begin{array}{r}12 \text { (c) } \\
4 \text { (c) }\end{array}$ & nd & $3(d)$ & nd & $2(d)$ & & \\
\hline $\begin{array}{l}\text { Untreated landfill } \\
\text { leachates }(\mu \mathrm{g} / \mathrm{l})\end{array}$ & & & nd & 4 (c) & & & & & & \\
\hline $\begin{array}{l}\text { Treated landfill } \\
\text { leachates }(\mu \mathrm{g} / \mathrm{l})\end{array}$ & & & nd & 5 (c) & & & & & & \\
\hline
\end{tabular}




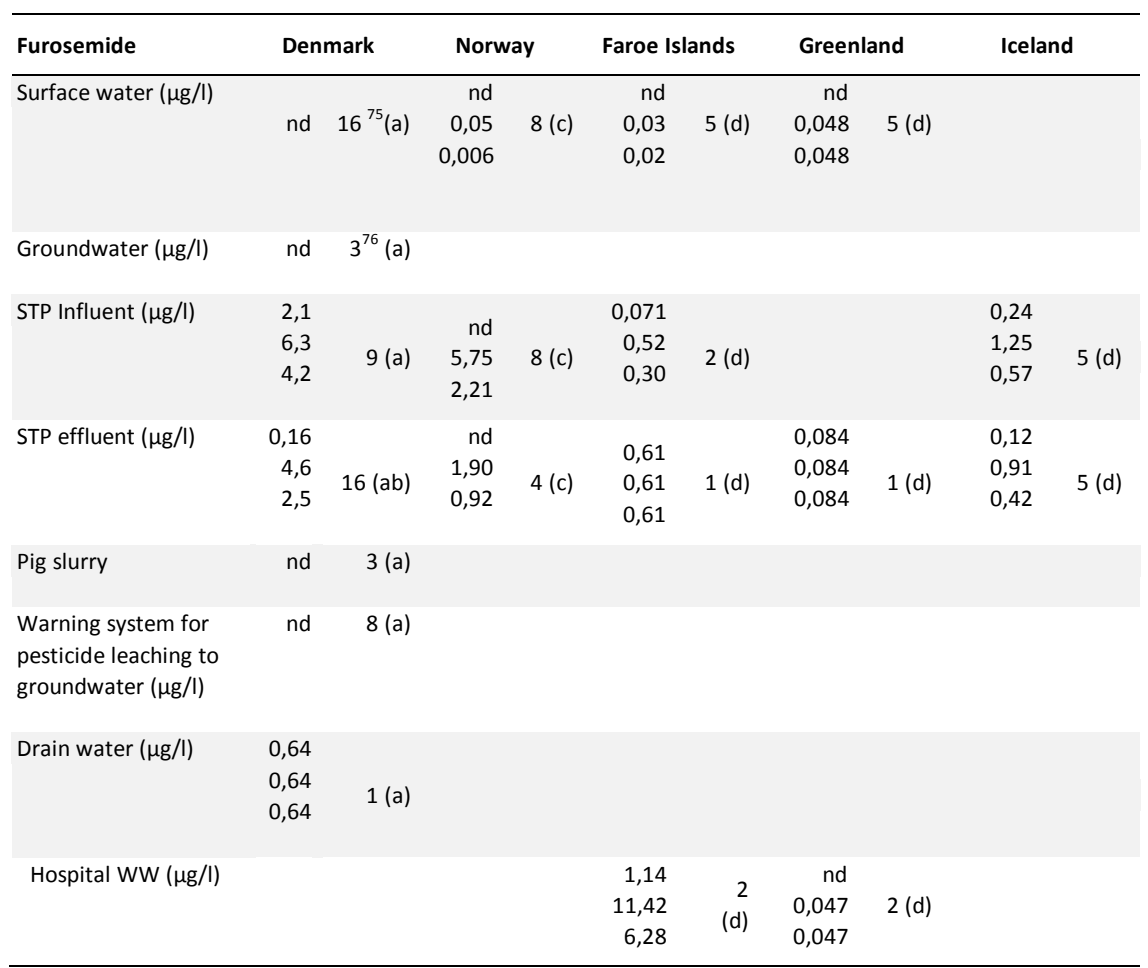

\section{References:}

a) (Mogensen, et al., 2008)

b) (Kjølholt, et al., 2003)

c) (Møskeland, et al., 2006)

d) (Huber, et al., NA)

\section{Conclusion:}

The compound was monitored in Denmark, Norway, Faroe Islands, Greenland and Iceland. It was detected in several matrices:

- Surface water in Norway, Greenland and Faroe Island

- STP sludge in all countries and in the large majority of all locations monitored. The values detected were higher in Greenland

- STP effluent water in all countries and in most samples analysed. Higher variations were observed in Denmark, the values detected were generally higher in Denmark than in the other countries

- Hospital waste water in all countries monitored. Values detected in Greenland were lower than in the other countries

- STP influent in all countries monitored

75 Upstream and downstream of fish farms

76 Including 2 measurements in groundwater at proximity of a hospital 


\subsubsection{Enalapril}

CAS nr.: 75847-73-3

Info: Angiotensin converting enzyme (ACE) inhibitor used in the treatment of hypertension and some types of chronic heart failure

Toxicity data: NA

$P N E C=\mathrm{NA}$
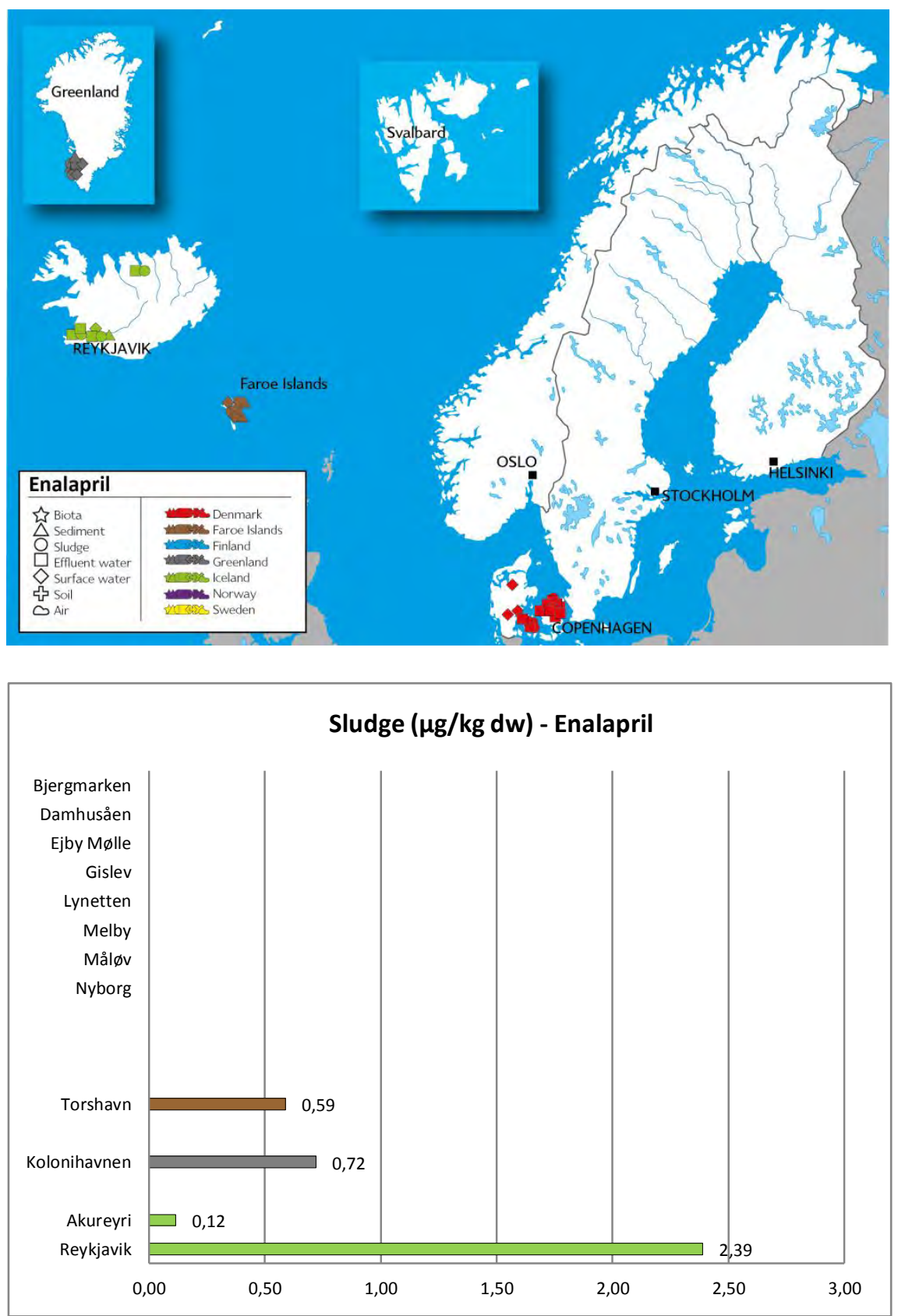

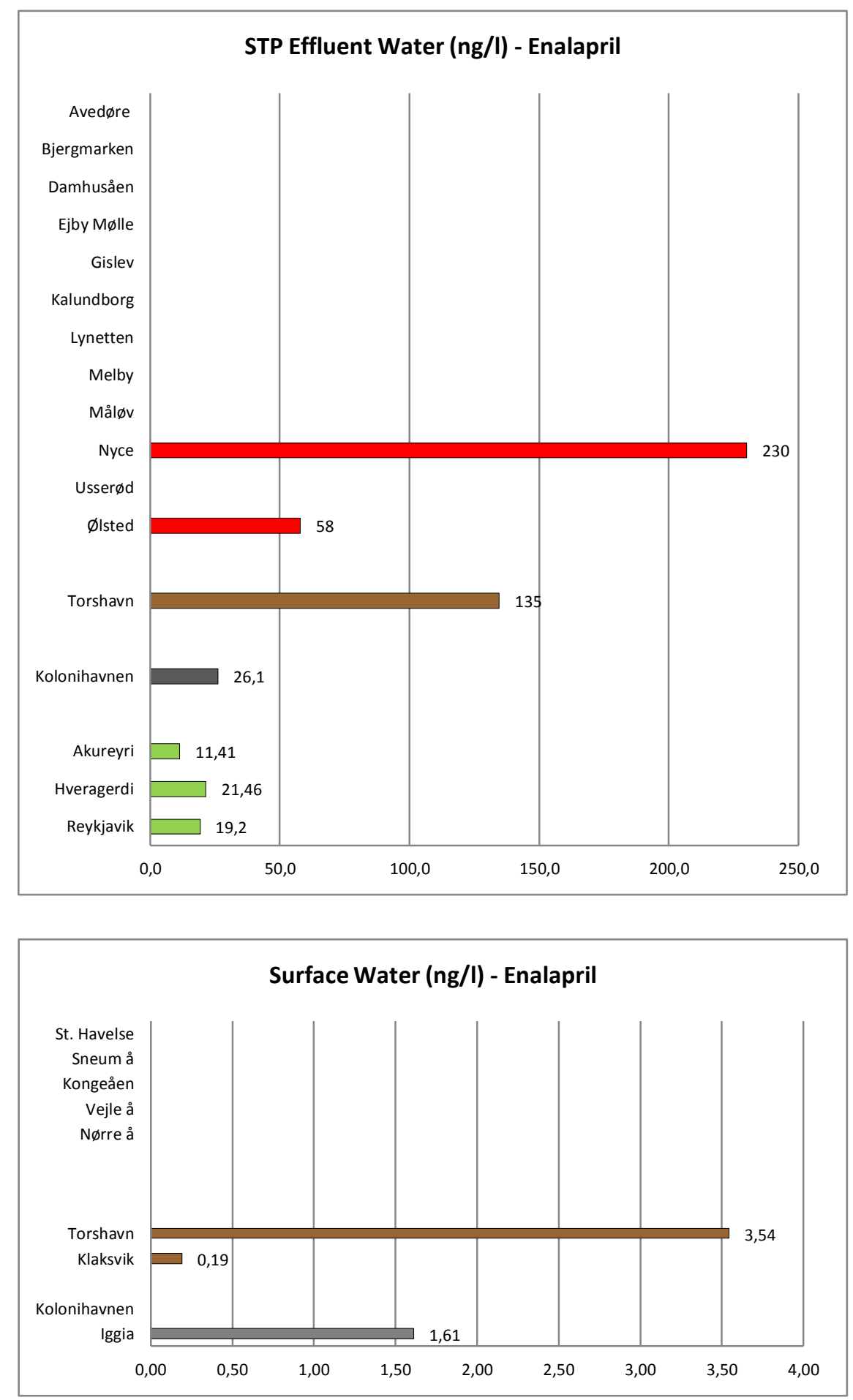


\begin{tabular}{|c|c|c|c|c|c|c|c|c|}
\hline \multirow{2}{*}{$\begin{array}{l}\text { Enalapril } \\
\text { Matrix }\end{array}$} & \multicolumn{2}{|c|}{ Denmark } & \multicolumn{2}{|c|}{ Faroe Islands } & \multicolumn{2}{|c|}{ Greenland } & \multicolumn{2}{|c|}{ Iceland } \\
\hline & $\begin{array}{r}\min \\
\max \\
A D\end{array}$ & $\begin{array}{l}\text { Nr.S } \\
\text { (ref) }\end{array}$ & $\begin{array}{r}\min \\
\max \\
A D\end{array}$ & $\begin{array}{l}\text { Nr.S } \\
\text { (ref) }\end{array}$ & $\begin{array}{r}\min \\
\max \\
A D\end{array}$ & $\begin{array}{l}\text { Nr.S } \\
\text { (ref) }\end{array}$ & $\begin{array}{r}\min \\
\max \\
A D\end{array}$ & $\begin{array}{l}\mathrm{Nr} . \mathrm{S} \\
\text { (ref) }\end{array}$ \\
\hline STP sludge $(\mu \mathrm{g} / \mathrm{kg} \mathrm{dw})$ & nd & $10(a)$ & $\begin{array}{l}0,13 \\
1,05 \\
0,59\end{array}$ & $2(c)$ & $\begin{array}{l}0,37 \\
1,07 \\
0,72\end{array}$ & 2 (c) & $\begin{array}{r}\text { nd } \\
2,39 \\
1,25\end{array}$ & $3(c)$ \\
\hline Sediment $(\mu \mathrm{g} / \mathrm{kg} \mathrm{dw})$ & & & nd & $3(c)$ & nd & 2 (c) & & \\
\hline Surface water (ng/l) & nd & $16^{77}(\mathrm{a})$ & $\begin{array}{l}0,19 \\
5,87 \\
2,71\end{array}$ & $5(c)$ & $\begin{array}{r}\text { nd } \\
2,98 \\
1,61\end{array}$ & 5 (c) & & \\
\hline Groundwater (ng/l) & nd & $3^{78}(a)$ & & & & & & \\
\hline STP Influent (ng/l) & $\begin{array}{l}170 \\
680 \\
370\end{array}$ & 9 (a) & $\begin{array}{r}32,1 \\
112 \\
72,3\end{array}$ & $2(c)$ & & & $\begin{array}{r}5,88 \\
26,9 \\
15,93\end{array}$ & 5 (c) \\
\hline STP effluent (ng/l) & $\begin{array}{r}\text { nd } \\
250 \\
140\end{array}$ & $15(a b)$ & $\begin{array}{l}135 \\
135 \\
135\end{array}$ & 1 (c) & $\begin{array}{l}26,1 \\
26,1 \\
26,1\end{array}$ & 1 (c) & $\begin{array}{l}11,4 \\
22,7 \\
17,0\end{array}$ & 5 (c) \\
\hline Pig slurry & nd & 4 (a) & & & & & & \\
\hline $\begin{array}{l}\text { Warning system for pesticide } \\
\text { leaching to groundwater ( } \mathrm{ng} / \mathrm{l})\end{array}$ & nd & 8 (a) & & & & & & \\
\hline Drain water (ng/l) & nd & 1 (a) & & & & & & \\
\hline Hospital WW (ng/l) & & & $\begin{array}{r}57,4 \\
120 \\
88,9\end{array}$ & $2(c)$ & $\begin{array}{l}113 \\
322 \\
217\end{array}$ & 2 (c) & & \\
\hline
\end{tabular}

\section{References:}

a) (Mogensen, et al., 2008)

b) (Kjølholt, et al., 2003)

c) (Huber, et al., NA)

\section{Conclusion:}

The compound was monitored in two studies in Denmark and one study in Faroe Islands, Greenland and Iceland. It was detected in several matrices:

- STP sludge in Faroe Islands, Greenland and Iceland, not in Denmark

- Surface water in Faroe Islands and Greenland, not in Denmark.

- STP effluent water in all countries. The values detected in Denmark and Faroe Islands were higher than in the other countries

- Hospital waste water in all countries monitored

- STP influent in all countries monitored

77 Upstream and downstream of fish farms

${ }^{78}$ Including 2 measurements in groundwater at proximity of a hospital 


\subsubsection{Metoprolol}

CAS nr.: 51384-51-1

Info: Selective beta-1 receptor blocker used in treatment of several diseases of the cardiovascular system, especially hypertension

Toxicity data: NA

$P N E C=8,8 \mu \mathrm{g} / \mathrm{l}$ (Schlabach, et al., 2007)

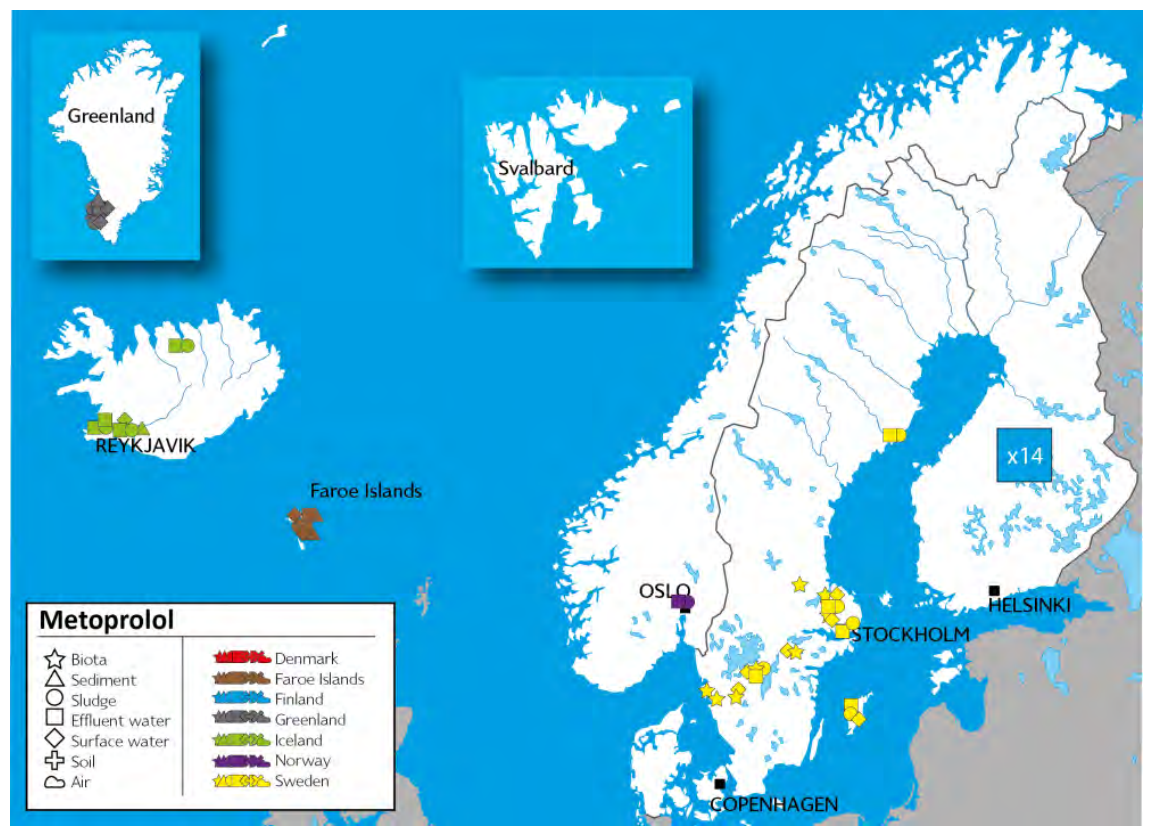




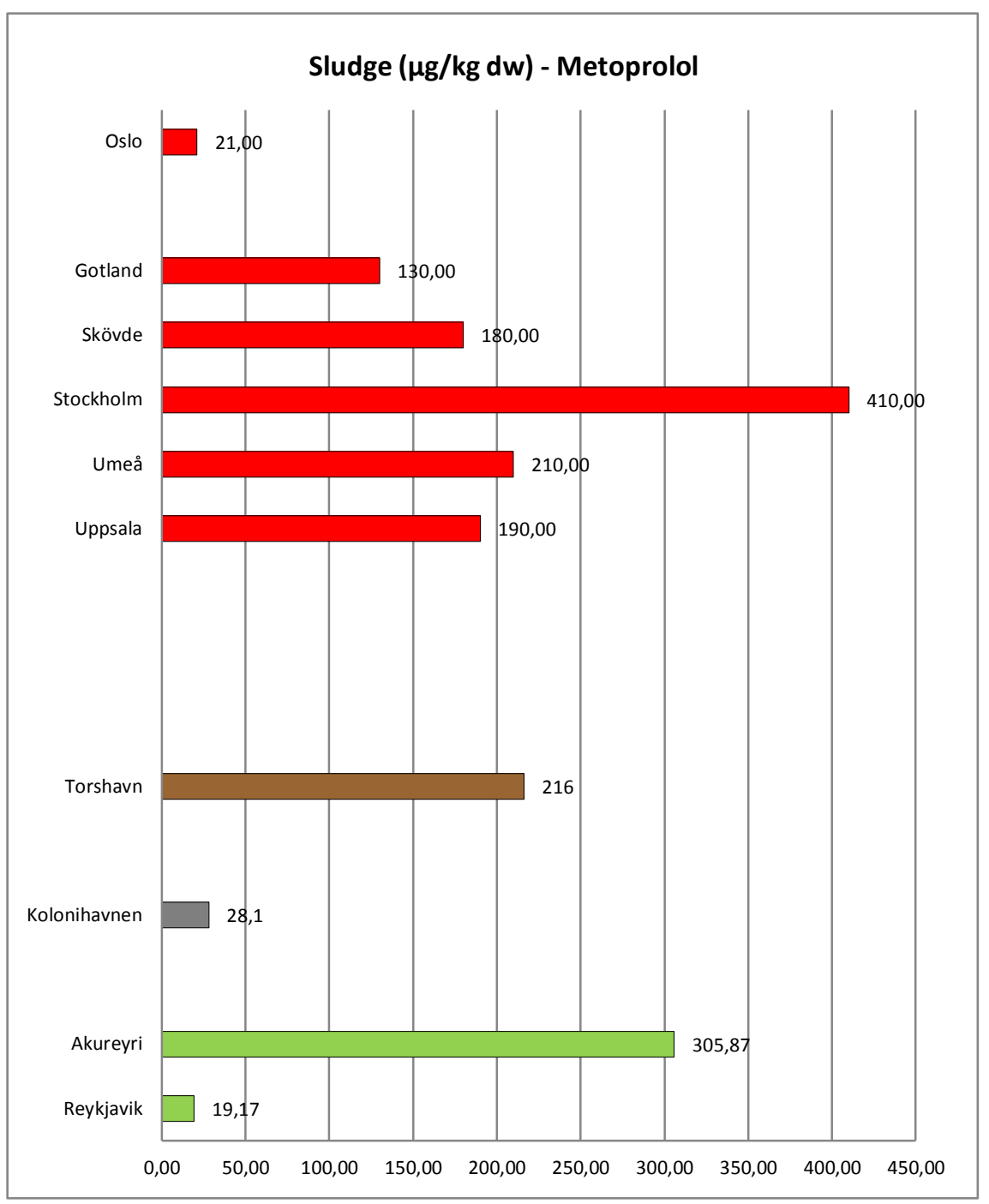



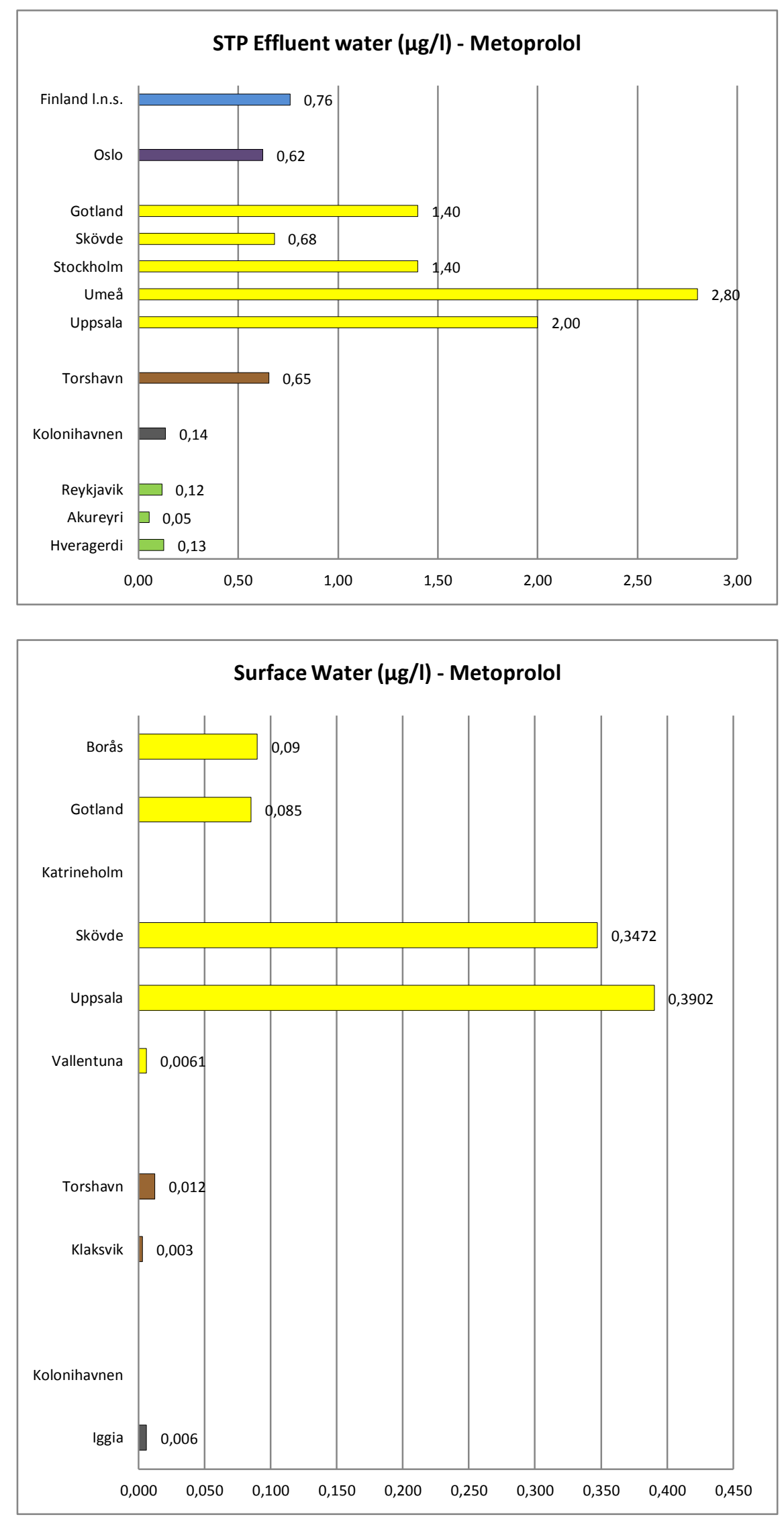


\begin{tabular}{|c|c|c|c|c|c|c|c|c|c|c|c|c|}
\hline \multirow{2}{*}{$\begin{array}{l}\text { Metoprolol } \\
\text { Matrix }\end{array}$} & \multicolumn{2}{|c|}{ Finland } & \multicolumn{2}{|c|}{ Norway } & \multicolumn{2}{|c|}{ Sweden } & \multicolumn{2}{|c|}{ Faroe Islands } & \multicolumn{2}{|c|}{ Greenland } & \multicolumn{2}{|c|}{ Iceland } \\
\hline & $\begin{array}{r}\min \\
\max \\
A D\end{array}$ & $\begin{array}{l}\text { Nr.S } \\
\text { (ref) }\end{array}$ & $\begin{array}{r}\min \\
\max \\
A D\end{array}$ & $\begin{array}{l}\text { Nr.S } \\
\text { (ref) }\end{array}$ & $\begin{array}{r}\min \\
\max \\
A D\end{array}$ & $\begin{array}{l}\text { Nr.S } \\
\text { (ref) }\end{array}$ & $\begin{array}{r}\min \\
\max \\
A D\end{array}$ & $\begin{array}{l}\text { Nr.S } \\
\text { (ref) }\end{array}$ & $\begin{array}{r}\min \\
\max \\
A D\end{array}$ & $\begin{array}{l}\text { Nr.S } \\
\text { (ref) }\end{array}$ & $\begin{array}{r}\min \\
\max \\
A D\end{array}$ & $\begin{array}{l}\text { Nr.S } \\
\text { (ref) }\end{array}$ \\
\hline Biota $(\mu \mathrm{g} / \mathrm{kg} w w)$ & & & & & nd & $15(d)$ & & & & & & \\
\hline $\begin{array}{l}\text { STP sludge } \\
(\mu \mathrm{g} / \mathrm{kg} \mathrm{dw})\end{array}$ & & & $\begin{array}{l}\text { nd } \\
21 \\
21\end{array}$ & 7 (c) & $\begin{array}{l}130 \\
410 \\
220\end{array}$ & $5(d)$ & $\begin{array}{l}108 \\
324 \\
216\end{array}$ & 2 (e) & $\begin{array}{l}14,7 \\
41,4 \\
28,1\end{array}$ & 2 (e) & $\begin{array}{r}19,2 \\
548,9 \\
210,3\end{array}$ & 3 (e) \\
\hline $\begin{array}{l}\text { Sediment } \\
(\mu \mathrm{g} / \mathrm{kg} \mathrm{dw})\end{array}$ & & & & & & & nd & 3 (e) & $\begin{array}{l}0,67 \\
7,39 \\
4,03\end{array}$ & 2 (e) & & \\
\hline Surface water $(\mu \mathrm{g} / \mathrm{l})$ & & & & & $\begin{array}{r}\text { nd } \\
1,20 \\
0,30\end{array}$ & 15 (d) & $\begin{array}{r}\text { nd } \\
0,02 \\
0,01\end{array}$ & 5 (e) & $\begin{array}{r}\text { nd } \\
0,01 \\
0,006\end{array}$ & $5(e)$ & & \\
\hline STP Influent ( $\mu \mathrm{g} / \mathrm{l})$ & $\begin{array}{r}N A \\
N A \\
1,06\end{array}$ & $14(a)$ & & & $\begin{array}{l}1,20 \\
6,80 \\
2,58\end{array}$ & 12 (d) & $\begin{array}{l}0,32 \\
0,40 \\
0,36\end{array}$ & 2 (e) & & & $\begin{array}{r}0,014 \\
0,18 \\
0,11\end{array}$ & 5 (e) \\
\hline STP effluent $(\mu \mathrm{g} / \mathrm{l})$ & $\begin{array}{r}\text { NA } \\
\text { NA } \\
0,76\end{array}$ & 14 (a) & $\begin{array}{l}0,37 \\
0,77 \\
0,59\end{array}$ & 6 (c) & $\begin{array}{l}0,13 \\
2,80 \\
1,62\end{array}$ & 14 (d) & $\begin{array}{l}0,65 \\
0,65 \\
0,65\end{array}$ & 1 (e) & $\begin{array}{l}0,14 \\
0,14 \\
0,14\end{array}$ & 1 (e) & $\begin{array}{l}0,05 \\
0,13 \\
0,09\end{array}$ & 5 (e) \\
\hline $\begin{array}{l}\text { Settleable particulate } \\
\text { material } \\
(\mathrm{ng} / \mathrm{g} \mathrm{dw})\end{array}$ & $\begin{array}{r}8,9 \\
104 \\
49,9\end{array}$ & 11 (b) & & & & & & & & & & \\
\hline Drinking water (ng/l) & & & & & $\begin{array}{r}\text { nd } \\
0,016 \\
0,010\end{array}$ & $6(d)$ & & & & & & \\
\hline Hospital WW $(\mu \mathrm{g} / \mathrm{l})$ & & & & & & & $\begin{array}{l}0,35 \\
0,81 \\
0,58\end{array}$ & 2 (e) & $\begin{array}{r}\text { nd } \\
0,25 \\
0,25\end{array}$ & $2(e)$ & & \\
\hline
\end{tabular}

\section{References:}

a) (Vieno, 2008)

b) (Lahti \& Oikari, 2011)

c) (Thomas, et al., 2007)

d) (Fick, et al., 2011)

e) (Huber, et al., NA)

\section{Conclusion:}

The compound was monitored in Finland, Norway, Faroe Islands, Greenland and Iceland. It was detected in several matrices:

- STP sludge in one sample in Norway, in Sweden, Faroe Islands, Greenland and Iceland. Lower values were detected in Norway and Greenland

- Sediment in Greenland, not Faroe Islands

- STP effluent in all countries. Lower values were detected in Iceland and Greenland 
- Surface water in all countries monitored. High variations were observed in values detected in Sweden

- STP influent in all countries monitored

\subsubsection{Amiloride}

CAS nr.: 2016-88-8

Info: Potassium-sparing diuretic

Toxicity data: NA

$P N E C=\mathrm{NA}$
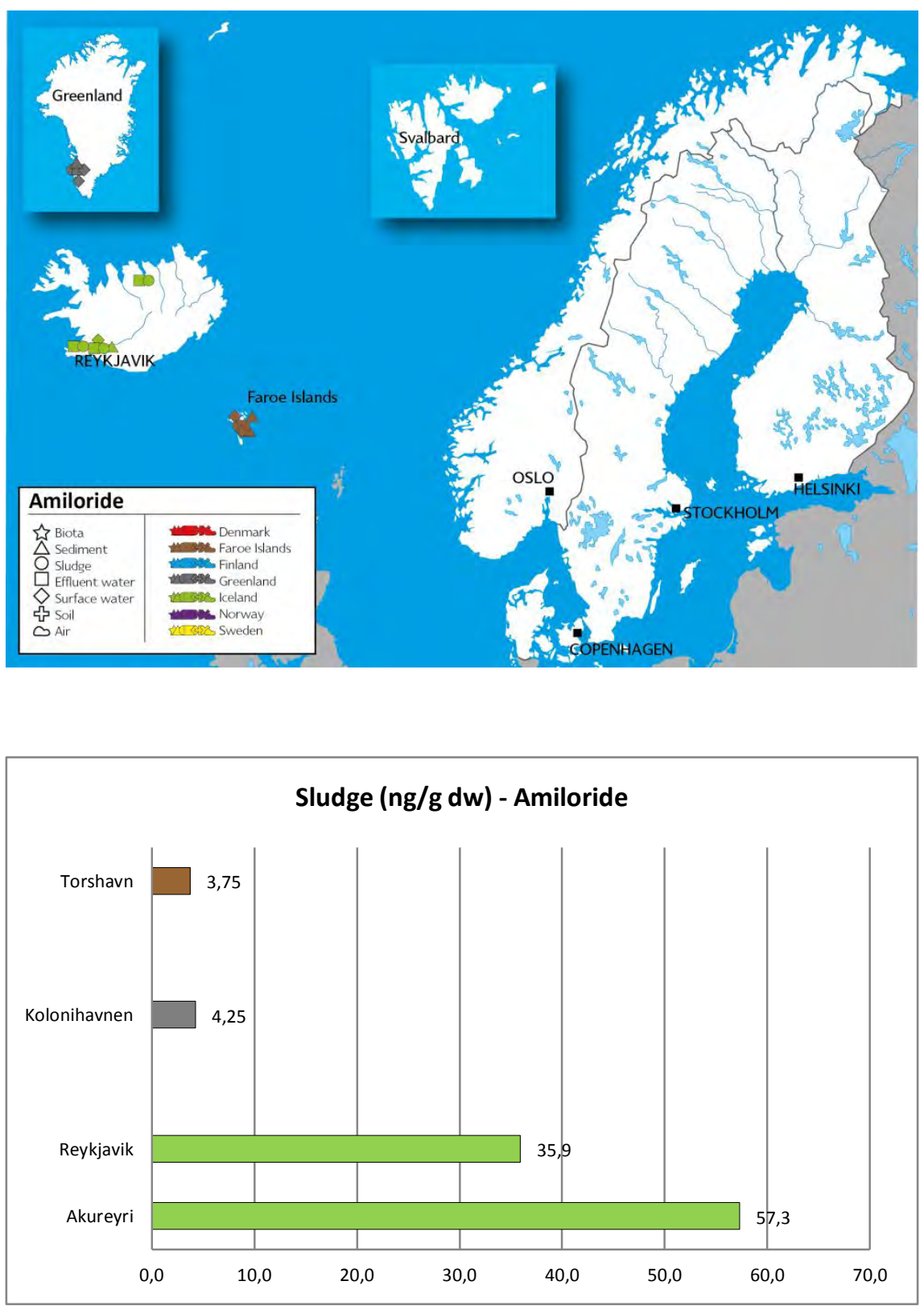


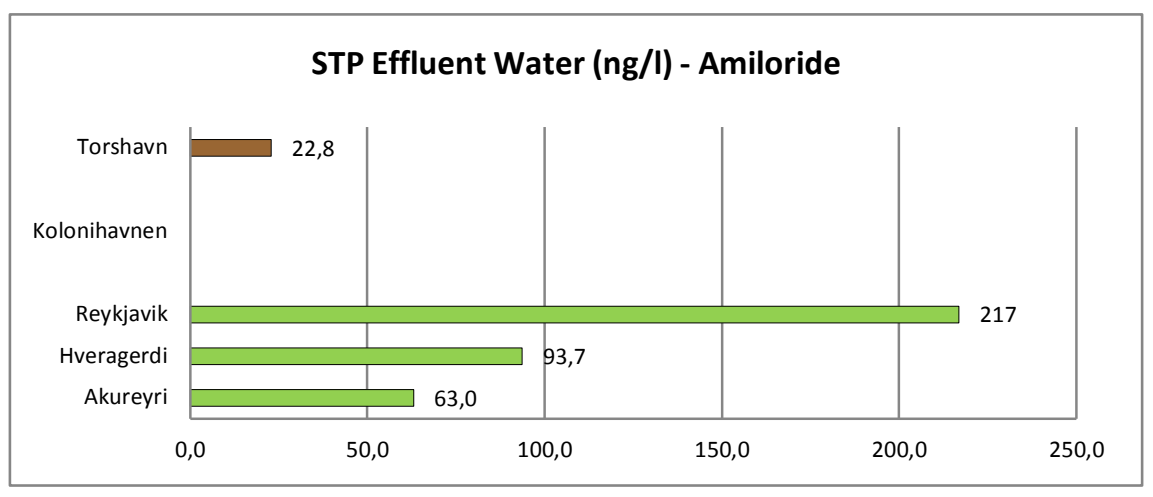

\begin{tabular}{|c|c|c|c|c|c|c|}
\hline $\begin{array}{l}\text { Amiloride } \\
\text { Matrix }\end{array}$ & \multicolumn{2}{|c|}{ Faroe Islands (a) } & \multicolumn{2}{|c|}{ Greenland (a) } & \multicolumn{2}{|c|}{ Iceland (a) } \\
\hline & $\begin{array}{r}\text { Min } \\
\max \\
A D\end{array}$ & $\begin{array}{l}\text { Nr.S } \\
\text { (ref) }\end{array}$ & $\begin{array}{r}\min \\
\max \\
A D\end{array}$ & $\begin{array}{l}\text { Nr.S } \\
\text { (ref) }\end{array}$ & $\begin{array}{r}\min \\
\max \\
A D\end{array}$ & $\begin{array}{l}\text { Nr.S } \\
\text { (ref) }\end{array}$ \\
\hline STP sludge (ng/g dw) & $\begin{array}{l}0,93 \\
6,56 \\
3,75\end{array}$ & 2 & $\begin{array}{l}1,83 \\
6,67 \\
4,25\end{array}$ & 2 & $\begin{array}{l}21,0 \\
93,6 \\
50,2\end{array}$ & 3 \\
\hline Sediment (ng/g dw) & $\begin{array}{r}\text { nd } \\
1,3 \\
0,94\end{array}$ & 3 & $\begin{array}{l}0,60 \\
0,79 \\
0,69\end{array}$ & 2 & & \\
\hline Surface water (ng/l) & $\begin{array}{r}53 \\
552,26 \\
301\end{array}$ & 5 & $\begin{array}{r}238 \\
397,6 \\
332\end{array}$ & 5 & & \\
\hline STP Influent (ng/l) & $\begin{array}{l}18,9 \\
25,6 \\
22,2\end{array}$ & 2 & & & $\begin{array}{r}30,6 \\
287,18 \\
149,4\end{array}$ & 5 \\
\hline STP effluent (ng/l) & $\begin{array}{l}22,8 \\
22,8 \\
22,8\end{array}$ & 1 & nd & 1 & $\begin{array}{r}40,9 \\
216,8 \\
106,0\end{array}$ & 5 \\
\hline Hospital WW (ng/l) & $\begin{array}{r}\text { nd } \\
8,27 \\
8,27\end{array}$ & 2 & nd & 2 & & \\
\hline
\end{tabular}

\section{References:}

a) (Huber, et al., NA)

\section{Conclusion:}

The compound was monitored in Faroe Islands, Greenland and Iceland. It was detected in several matrices:

- STP sludge in all three countries. While concentrations were similar in Faroe Islands and Greenland, Iceland showed values which were ten times higher

- Sediment in Faroe Islands and Greenland.

- Surface water in Faroe Islands and Greenland

- Effluent water in Faroe Islands and Iceland 
- STP influent in Faroe Islands and Iceland

- Hospital waste water in Faroe Islands

\subsubsection{Candesartan}

CAS nr.: 139481-59-7

Info: Angiotensin II receptor antagonists indicated for the treatment of hypertension

Toxicity data: NA

$P N E C=\mathrm{NA}$
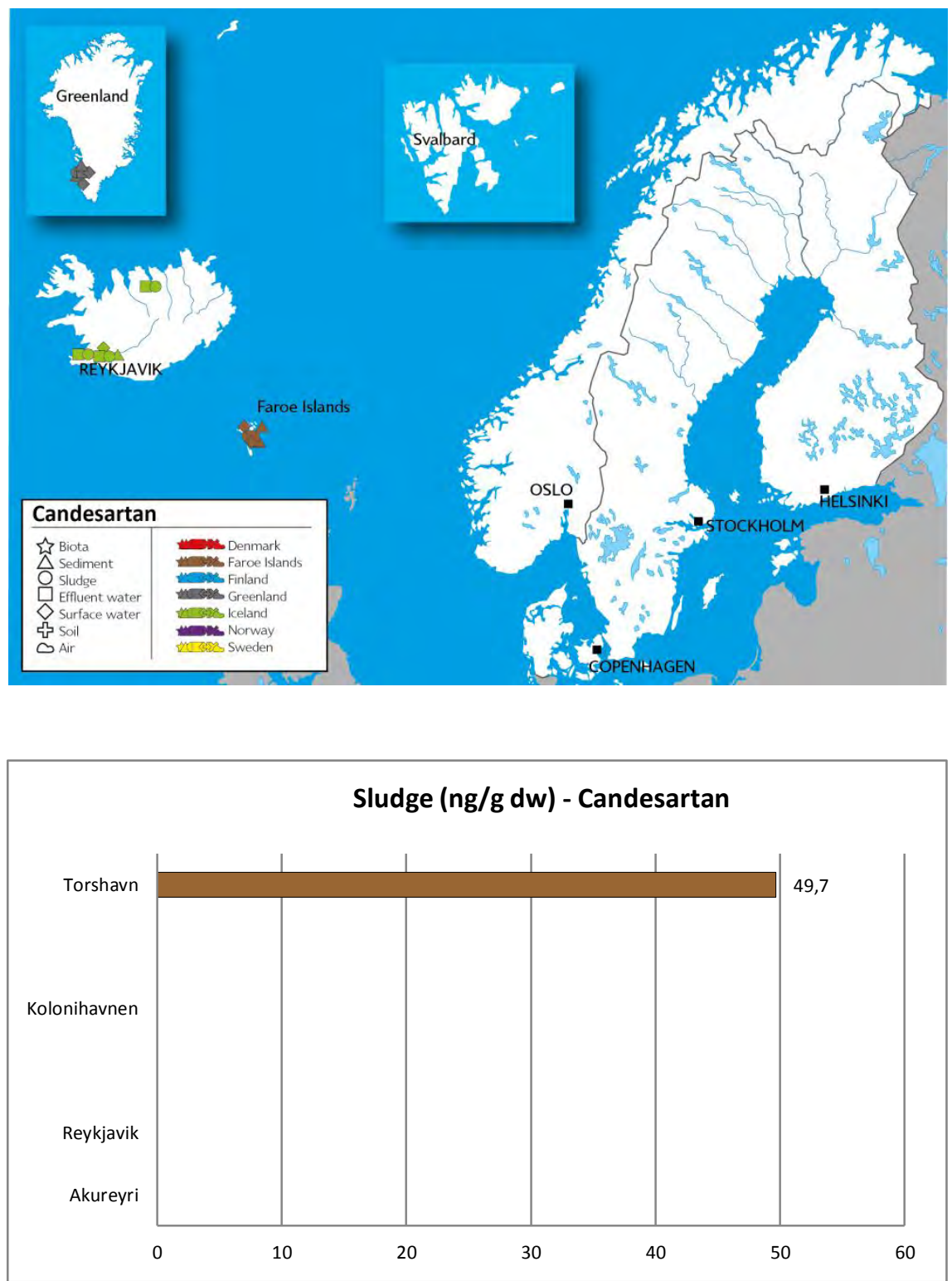


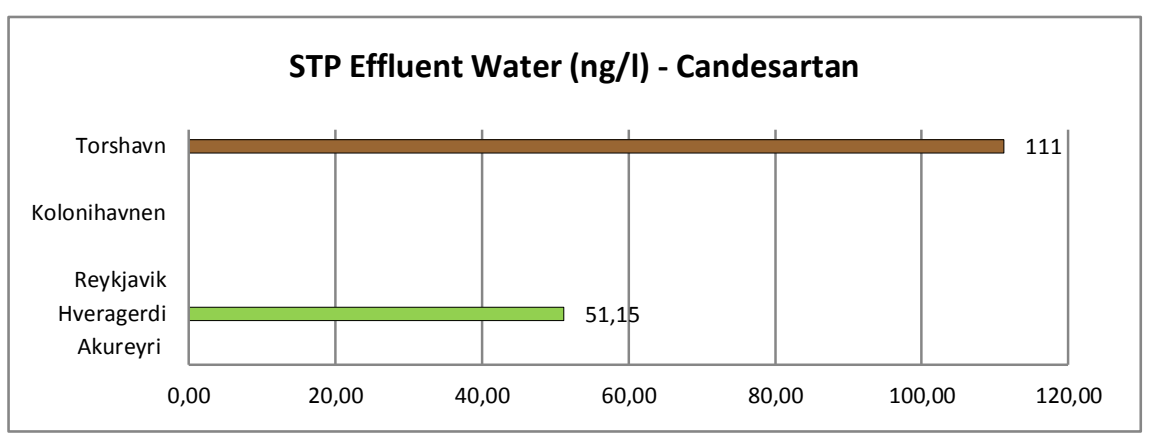

\begin{tabular}{|c|c|c|c|c|c|c|}
\hline \multirow{2}{*}{$\begin{array}{l}\text { Candesartan } \\
\text { Matrix }\end{array}$} & \multicolumn{2}{|c|}{ Faroe Islands (a) } & \multicolumn{2}{|c|}{ Greenland (a) } & \multicolumn{2}{|c|}{ Iceland (a) } \\
\hline & $\begin{array}{r}\min \\
\max \\
A D\end{array}$ & $\begin{array}{l}\text { Nr.S } \\
\text { (ref) }\end{array}$ & $\begin{array}{r}\min \\
\max \\
A D\end{array}$ & $\begin{array}{l}\text { Nr.S } \\
\text { (ref) }\end{array}$ & $\begin{array}{r}\min \\
\max \\
A D\end{array}$ & $\begin{array}{l}\text { Nr.S } \\
\text { (ref) }\end{array}$ \\
\hline STP sludge (ng/g dw) & $\begin{array}{r}\text { nd } \\
49,7 \\
49,7\end{array}$ & 2 & nd & 2 & nd & 3 \\
\hline Sediment (ng/g dw) & nd & 3 & nd & 2 & & \\
\hline Surface water (ng/l) & $\begin{array}{r}\text { nd } \\
5,34 \\
4,63\end{array}$ & 5 & nd & 5 & & \\
\hline STP Influent (ng/l) & $\begin{array}{r}53,8 \\
60,3 \\
57\end{array}$ & 2 & & & $\begin{array}{r}\text { nd } \\
36,6 \\
30,7\end{array}$ & 5 \\
\hline STP effluent (ng/l) & $\begin{array}{l}111 \\
111 \\
111\end{array}$ & 1 & nd & 1 & $\begin{array}{r}\mathrm{Nd} \\
54,3 \\
51,1\end{array}$ & 4 \\
\hline Hospital WW (ng/l) & $\begin{array}{l}142 \\
251 \\
196\end{array}$ & 2 & nd & 2 & & \\
\hline
\end{tabular}

\section{References:}

a) (Huber, et al., NA)

\section{Conclusion:}

The compound was monitored in Faroe Islands, Greenland and Iceland.

It was detected in several matrices in Faroe Islands and Iceland but it was never detected in Greenland:

- STP sludge in Faroe Islands

- Surface water in Faroe Islands

- STP effluent in Faroe Islands and Iceland. Concentrations in the Faroe Islands were higher.

- STP influent in Faroe Islands and Iceland

- Hospital waste water in Faroe Islands 


\subsubsection{Hydrochlorothiazide}

CAS nr.: 58-93-5

Info: Diuretic

Toxicity data: NA

$P N E C=\mathrm{NA}$
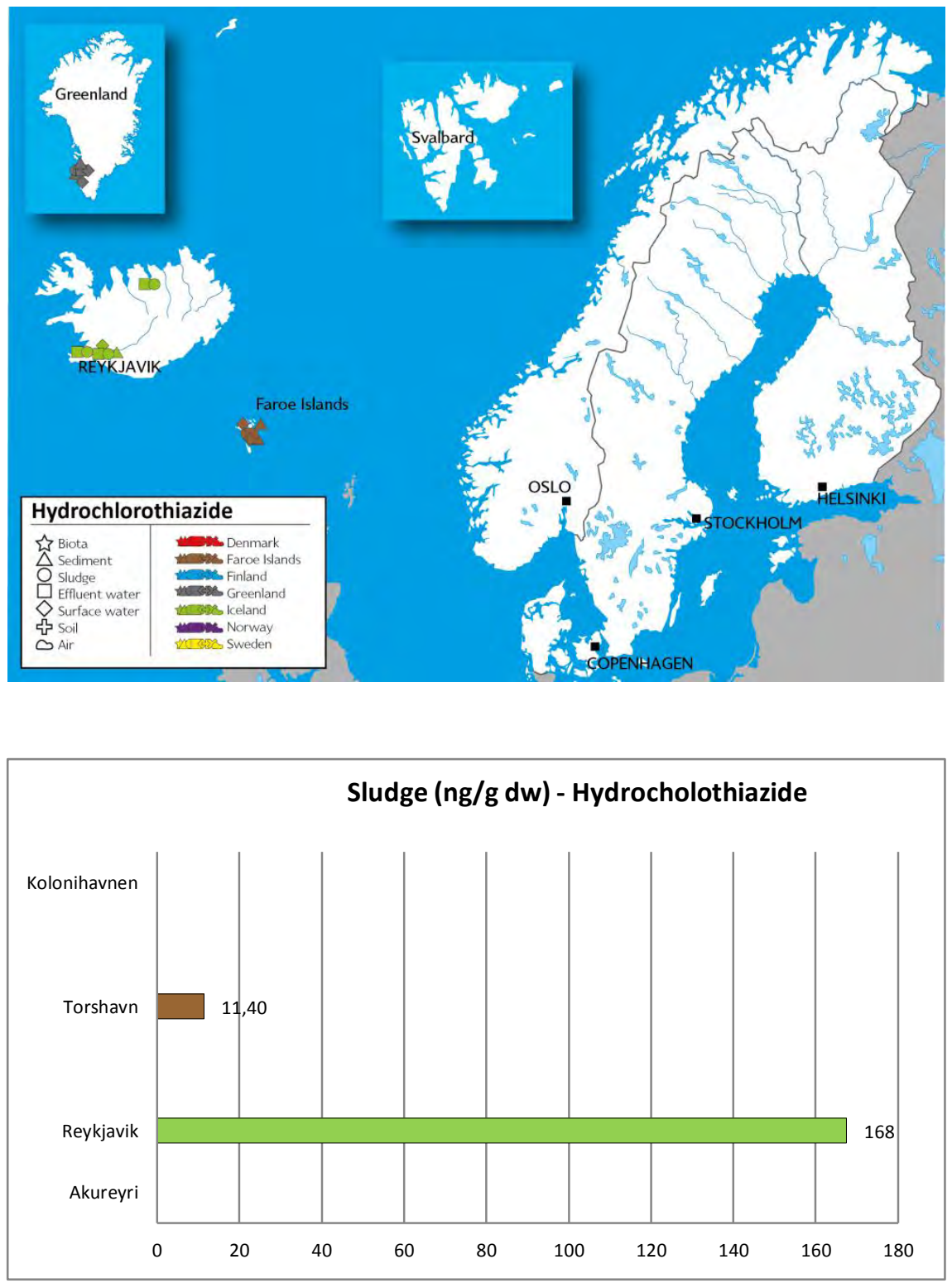


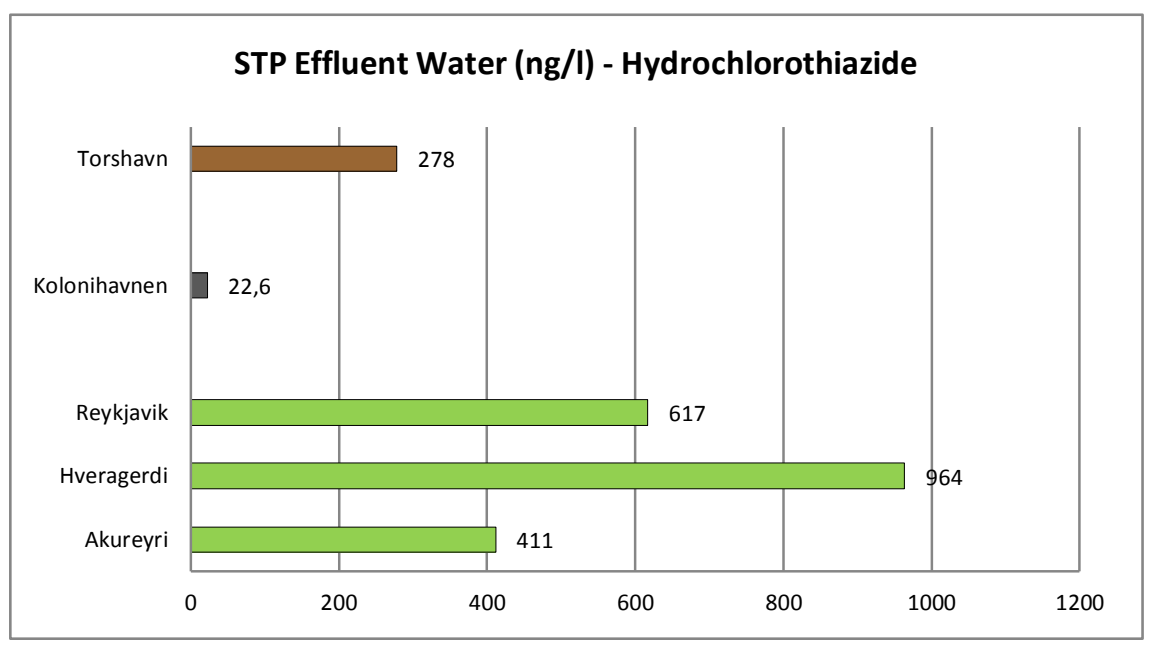

\begin{tabular}{|c|c|c|c|c|c|c|}
\hline \multirow{2}{*}{$\begin{array}{l}\text { Hydrochlorothiazide } \\
\text { Matrix }\end{array}$} & \multicolumn{2}{|c|}{ Faroe Islands (a) } & \multicolumn{2}{|c|}{ Greenland (a) } & \multicolumn{2}{|c|}{ Iceland (a) } \\
\hline & $\min$ & Nr.S & $\min$ & Nr.S & $\min$ & Nr.S \\
\hline & $\max$ & (ref) & $\max$ & (ref) & $\max$ & (ref) \\
\hline & $A D$ & & $A D$ & & $A D$ & \\
\hline \multirow[t]{3}{*}{ STP sludge (ng/g dw) } & 7,52 & \multirow[b]{3}{*}{2} & \multirow{3}{*}{ nd } & \multirow{3}{*}{2} & nd & \\
\hline & 15,3 & & & & 167,5 & \\
\hline & 11,40 & & & & 167,5 & 3 \\
\hline Sediment (ng/g dw) & nd & 3 & nd & 2 & & \\
\hline Surface water (ng/l) & nd & 5 & nd & 5 & & \\
\hline \multirow[t]{3}{*}{ STP Influent (ng/l) } & 87,9 & 2 & & & 258 & \\
\hline & 90,4 & & & & 1258 & 5 \\
\hline & 89,1 & & & & 616 & \\
\hline \multirow[t]{3}{*}{ STP effluent (ng/l) } & 278 & & 22,6 & & 410 & \\
\hline & 278 & 1 & 22,6 & 1 & 983,9 & 5 \\
\hline & 278 & & 22,6 & & 673,5 & \\
\hline \multirow[t]{3}{*}{ Hospital WW (ng/l) } & 345 & & 6,26 & & & \\
\hline & 345 & 2 & 6,42 & 2 & & \\
\hline & 345 & & 6,34 & & & \\
\hline
\end{tabular}

\section{References:}

a) (Huber, et al., NA)

\section{Conclusion:}

The compound has been monitored in one international study covering Faroe Islands, Greenland, and Iceland. It has been detected in several matrices:

- STP sludge in Faroe Islands and Iceland. Highest values were detected in Iceland

- STP effluent water in all three countries. The lowest concentrations were detected in Greenland 
- STP influent in Faroe Islands and Iceland

- Hospital waste water in the Faroe Islands and Greenland

\subsubsection{Losartan}

CAS nr.: 124750-99-8

Info: Angiotensin II receptor antagonist used to treat hypertension Toxicity data: NA

$P N E C=\mathrm{NA}$
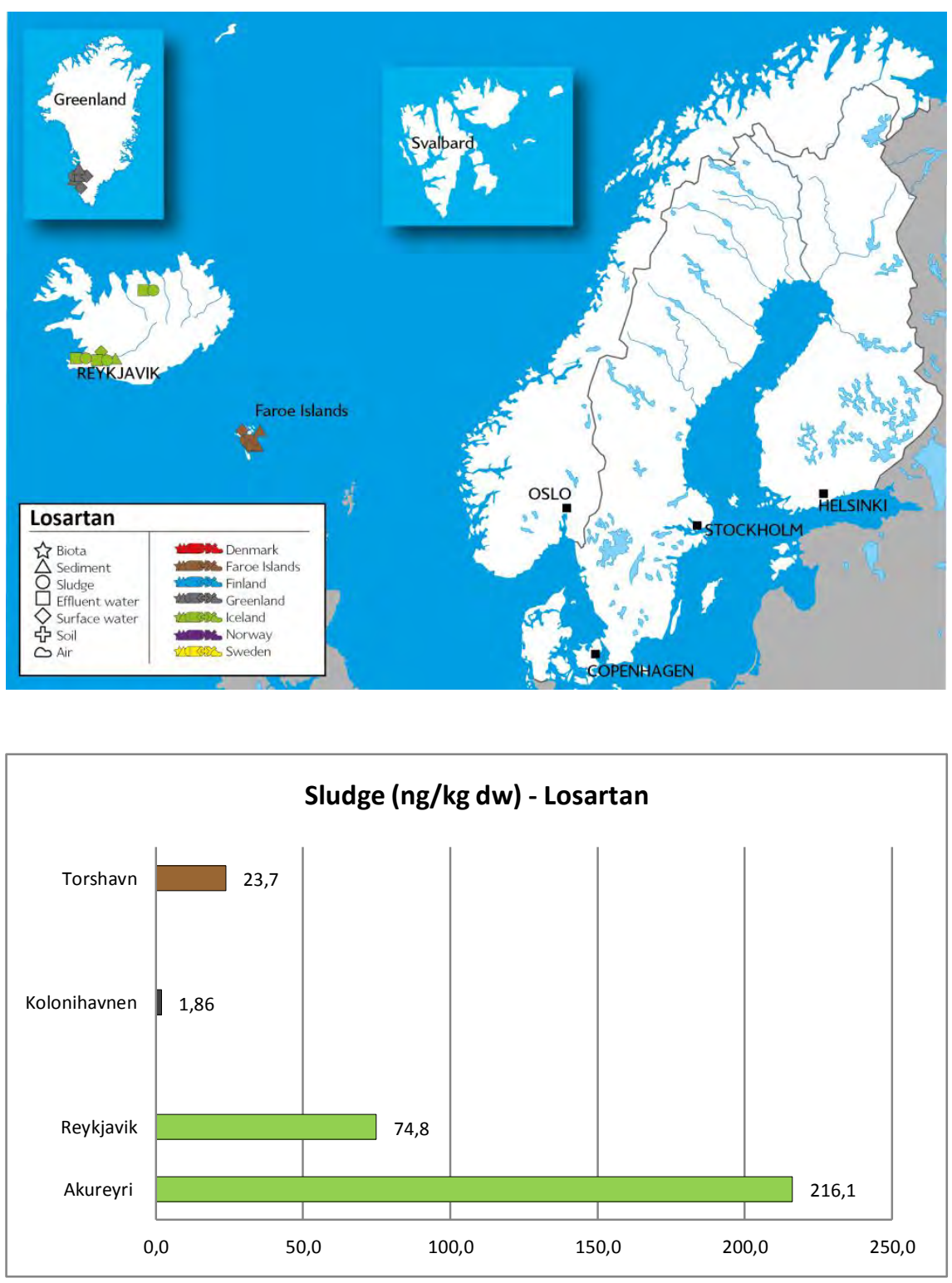


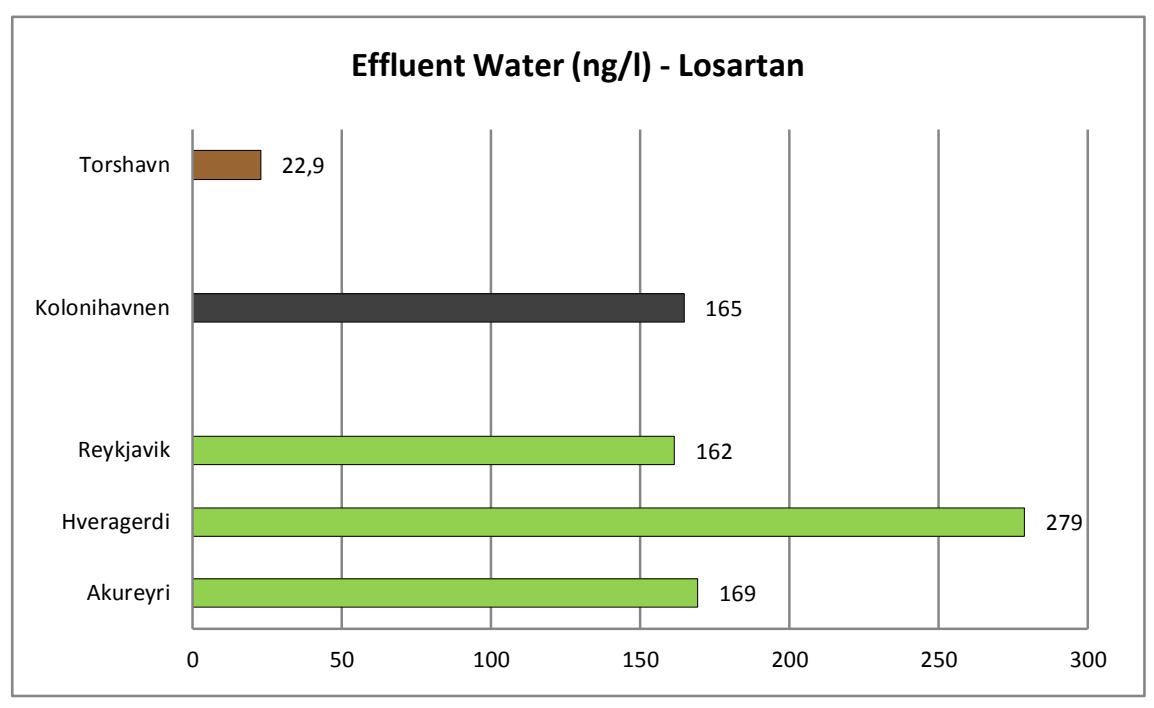

\begin{tabular}{|c|c|c|c|c|c|c|}
\hline $\begin{array}{l}\text { Losartan } \\
\text { Matrix }\end{array}$ & \multicolumn{2}{|c|}{ Faroe Islands (a) } & \multicolumn{2}{|c|}{ Greenland (a) } & \multicolumn{2}{|c|}{ Iceland (a) } \\
\hline Matrix & $\begin{array}{r}\min \\
\max \\
A D\end{array}$ & $\begin{array}{l}\text { Nr.S } \\
\text { (ref) }\end{array}$ & $\begin{array}{r}\min \\
\max \\
A D\end{array}$ & $\begin{array}{l}\text { Nr.S } \\
\text { (ref) }\end{array}$ & $\begin{array}{r}\min \\
\max \\
A D\end{array}$ & $\begin{array}{l}\text { Nr.S } \\
\text { (ref) }\end{array}$ \\
\hline STP sludge (ng/g dw) & $\begin{array}{r}14,4 \\
33 \\
23,7\end{array}$ & 2 & $\begin{array}{l}1,80 \\
1,92 \\
1,86\end{array}$ & 2 & $\begin{array}{r}39,9 \\
392,4 \\
169,0\end{array}$ & 3 \\
\hline Sediment ( $\mathrm{ng} / \mathrm{g} \mathrm{dw}$ ) & nd & 3 & nd & 2 & & \\
\hline Surface water (ng/l) & $\begin{array}{r}\text { nd } \\
4,60 \\
4,33\end{array}$ & 5 & $\begin{array}{r}\text { nd } \\
5,03 \\
5,03\end{array}$ & 5 & & \\
\hline STP Influent (ng/l) & $\begin{array}{l}25,8 \\
98,5 \\
62,2\end{array}$ & 2 & & & $\begin{array}{r}189 \\
586,3 \\
298\end{array}$ & 5 \\
\hline STP effluent (ng/l) & $\begin{array}{l}22,9 \\
22,9 \\
22,9\end{array}$ & 1 & $\begin{array}{l}165 \\
165 \\
165\end{array}$ & 1 & $\begin{array}{l}161,5 \\
281,2 \\
211,6\end{array}$ & 5 \\
\hline Hospital WW (ng/l) & $\begin{array}{l}159 \\
292 \\
226\end{array}$ & 2 & $\begin{array}{l}21,5 \\
74,3 \\
47,9\end{array}$ & 2 & & \\
\hline
\end{tabular}

\section{References:}

a) (Huber, et al., NA)

\section{Conclusion:}

The compound has been monitored in one international study covering Faroe Islands, Greenland, and Iceland. It has been detected in several matrices:

- STP sludge in all three countries. Highest values were detected in Iceland

- Surface water in all the countries. monitored 
- STP effluent water in all three countries. The lowest concentrations were detected in Faroe Islands

- STP influent in Faroe Islands and Iceland. The highest values were detected in Iceland

- Hospital waste water in Greenland and the Faroe Islands

\subsection{Compounds monitored in at least two studies}

\subsubsection{Acebutol}

CAS nr.: 37517-30-9

Info: Cardioselective beta blocker with ISA (intrinsic sympathomimetic) activity

Toxicity data: NA

$P N E C=\mathrm{NA}$

\begin{tabular}{lcc}
\hline Acebutol & Finland & \\
\hline Matrix & $\min$ & Nr.S \\
& (ref) \\
& $\mathrm{AD}$ & \\
STP effluent $(\mu \mathrm{g} / \mathrm{l})$ & $\mathrm{NA}$ & 14 (a) \\
& $\mathrm{NA}$ & \\
& 0,14 & $14(\mathrm{a})$ \\
Influent water $(\mu \mathrm{g} / \mathrm{l})$ & $\mathrm{NA}$ & \\
& $\mathrm{NA}$ & 11 (b) \\
Settleable particulate material $(\mathrm{ng} / \mathrm{g} \mathrm{dw})$ & 0,34 & $\mathrm{nd}$ \\
\end{tabular}

\section{References:}

a) (Vieno, 2008)

b) (Lahti \& Oikari, 2011)

\section{Conclusion:}

The compound was monitored in two studies in Finland and was detected in all matrices monitored: STP influent and effluent and settleable particulate matter. 


\subsubsection{Bisoprolol}

CAS nr.: 66722-44-9

Info: Selective type beta- 1 adrenergic receptor blocker

Toxicity data: $\mathrm{NA}$

$P N E C=\mathrm{NA}$

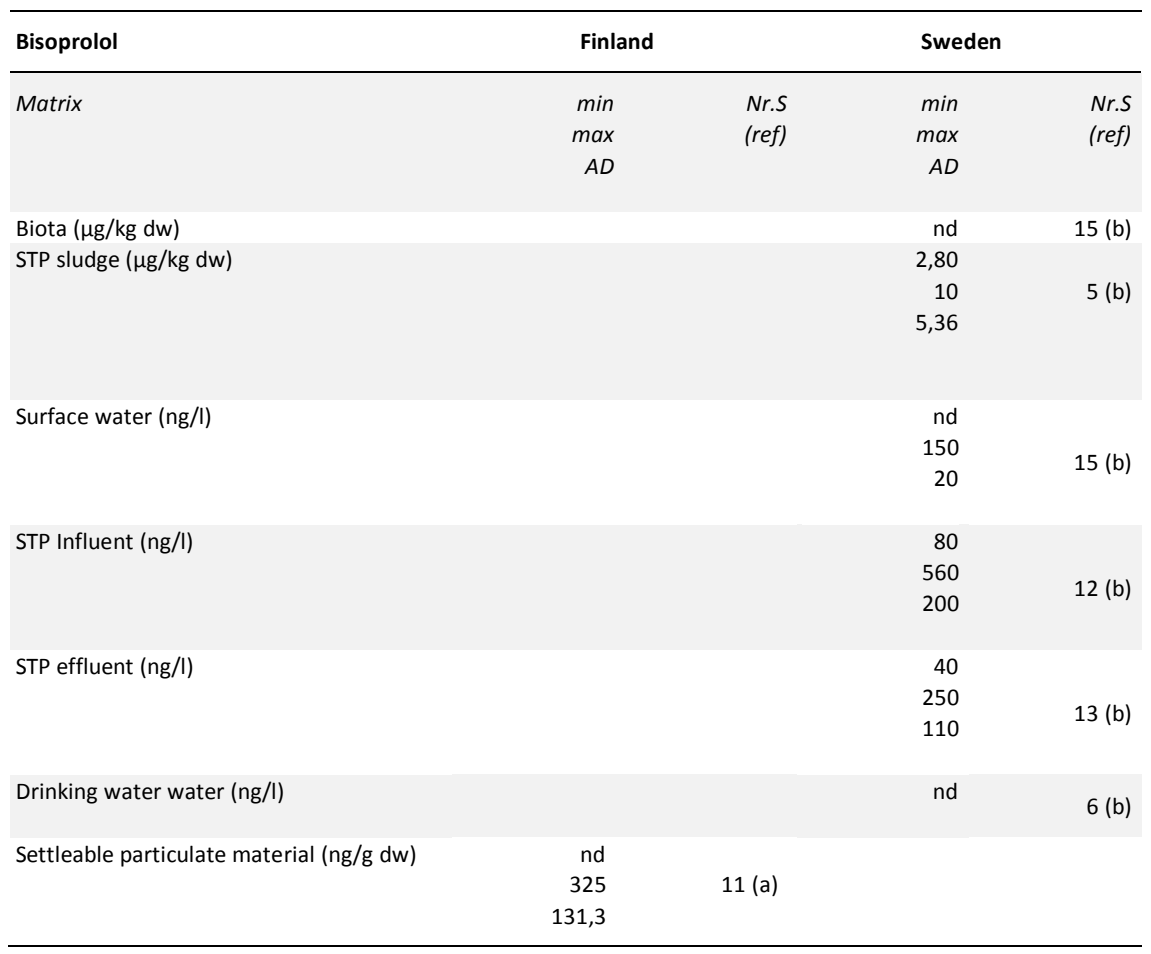

\section{References:}

a) (Lahti \& Oikari, 2011)

b) (Fick, et al., 2011)

\section{Conclusion:}

The compound was monitored in Finland and Sweden. It was detected in Sweden in surface water, STP sludge, effluent and influent as well as in settleable particulate material in Finland. 


\subsubsection{Felodipine}

CAS nr.: 72509-76-3

Info: Calcium channel blocker (calcium antagonist) used to control hypertension

Toxicity data: NA

$P N E C=\mathrm{NA}$

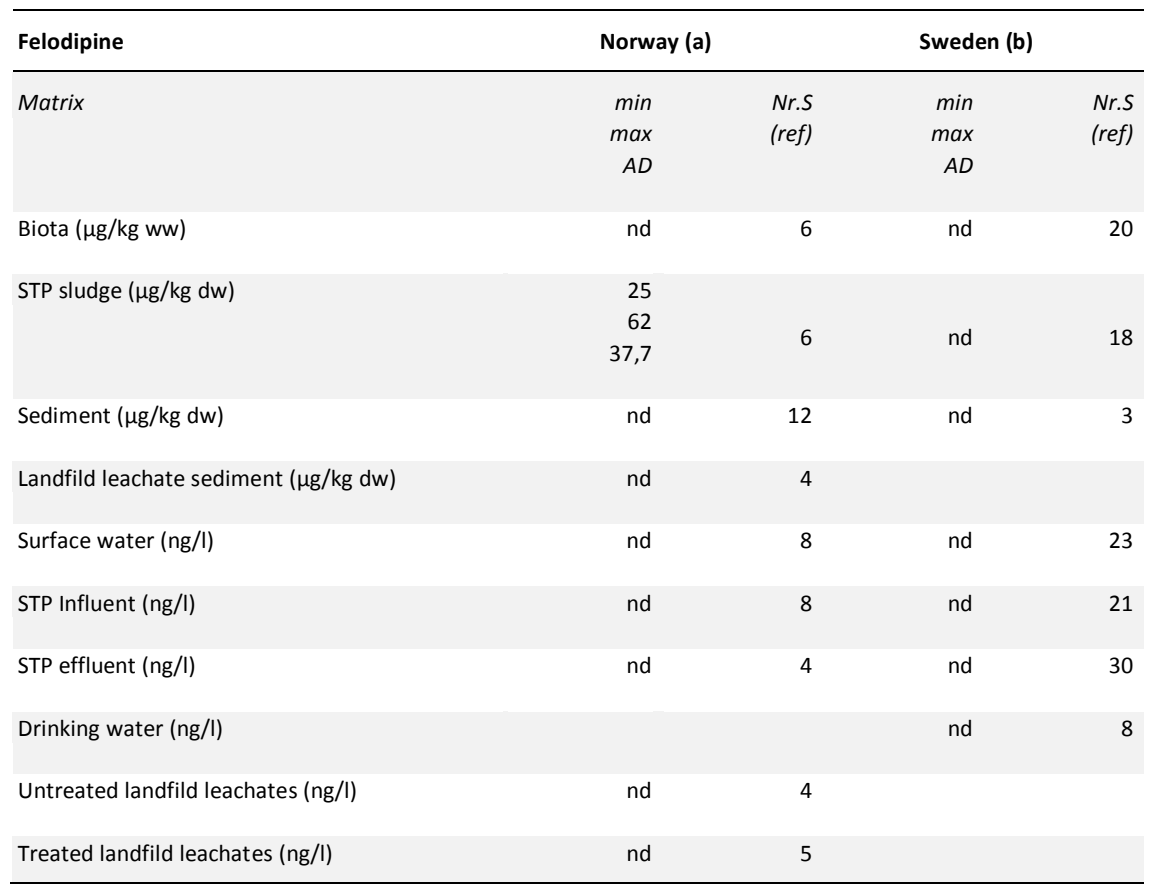

\section{References:}

a) (Møskeland, et al., 2006)

b) (Kjølholt, et al., 2003)

\section{Conclusion:}

The compound was monitored in Norway and Sweden. It was only detected in STP sludge in Norway. The compound was not detected in any other matrice in either country. 


\subsubsection{Sotalol}

CAS nr.: 959-24-0

Info: Used in individuals with rhythm disturbances (cardiac arrhythmias) of the heart, and to treat hypertension

Toxicity data: NA

$P N E C=\mathrm{NA}$

\begin{tabular}{|c|c|c|}
\hline Sotalol & & \\
\hline \multirow[t]{3}{*}{ Matrix } & $\min$ & Nr.S \\
\hline & $\max$ & (ref) \\
\hline & $A D$ & \\
\hline \multirow[t]{3}{*}{ STP Influent ( $\mu \mathrm{g} / \mathrm{I})$} & nd & \multirow{3}{*}{14 (a) } \\
\hline & 0,83 & \\
\hline & 0,83 & \\
\hline \multirow[t]{3}{*}{ Effluent water ( $\mu \mathrm{g} / \mathrm{l})$} & nd & \multirow{3}{*}{14 (a) } \\
\hline & 0,28 & \\
\hline & 0,28 & \\
\hline Settleable particulate material ( $\mathrm{ng} / \mathrm{g} \mathrm{dw}$ ) & nd & 11 (b) \\
\hline
\end{tabular}

\section{References:}

a) (Vieno, 2008)

b) (Lahti \& Oikari, 2011)

\section{Conclusion:}

The compound was monitored in two studies in Finland and was detected in one STP influent sample and one STP effluent sample.

\subsection{Compounds monitored in one study}

\begin{tabular}{|c|c|c|c|c|c|c|c|c|}
\hline & $\begin{array}{r}\text { Alfuzosin } \\
(81403-80-7\end{array}$ & $\begin{array}{r}\text { Cilazapril } \\
(88768-40-5)\end{array}$ & $\begin{array}{r}\text { Diltiazem } \\
(42399-41-7)\end{array}$ & $\begin{array}{r}\text { Eprosartan } \\
(133040-01-4)\end{array}$ & $\begin{array}{r}\text { Irbesartan } \\
(138402-11-6)\end{array}$ & $\begin{array}{r}\text { Telmisartan } \\
(144701-48-4)\end{array}$ & $\begin{array}{r}\text { Verapamil } \\
(52-53-9)\end{array}$ & \\
\hline Matrix & $\begin{array}{r}\min \\
\max \\
A D\end{array}$ & $\begin{array}{r}\min \\
\max \\
A D\end{array}$ & $\begin{array}{r}\min \\
\max \\
A D\end{array}$ & $\begin{array}{r}\min \\
\max \\
A D\end{array}$ & $\begin{array}{r}\min \\
\max \\
A D\end{array}$ & $\begin{array}{r}\min \\
\max \\
A D\end{array}$ & $\begin{array}{r}\min \\
\max \\
A D\end{array}$ & Nr.S \\
\hline $\begin{array}{l}\text { Biota } \\
\text { (ng/g ww) }\end{array}$ & $\begin{array}{r}\text { nd } \\
0,2 \\
0,14\end{array}$ & nd & nd & nd & $\begin{array}{r}\text { nd } \\
1,3 \\
0,91\end{array}$ & nd & nd & 15 \\
\hline $\begin{array}{l}\text { STP sludge } \\
\text { (ng/g dw) }\end{array}$ & $\begin{array}{r}10 \\
34 \\
21,20\end{array}$ & $\begin{array}{r}1,10 \\
2,6 \\
1,54\end{array}$ & $\begin{array}{r}\text { nd } \\
0,80 \\
0,80\end{array}$ & $\begin{array}{r}\text { nd } \\
14 \\
12,67\end{array}$ & $\begin{array}{r}8,70 \\
280 \\
93,54\end{array}$ & $\begin{array}{r}\text { nd } \\
1400 \\
540\end{array}$ & $\begin{array}{l}\text { nd } \\
18 \\
18\end{array}$ & 5 \\
\hline $\begin{array}{l}\text { STP effluent } \\
\text { (ng/l) }\end{array}$ & $\begin{array}{r}18 \\
110 \\
55,23\end{array}$ & $\begin{array}{c}\text { nd } \\
28 \\
8,7\end{array}$ & $\begin{array}{r}11 \\
100 \\
42,69\end{array}$ & $\begin{array}{r}12 \\
870 \\
239,7\end{array}$ & $\begin{array}{r}19 \\
1100 \\
416,1\end{array}$ & $\begin{array}{r}\text { nd } \\
250 \\
113,8\end{array}$ & $\begin{array}{r}\text { nd } \\
29 \\
17,33\end{array}$ & 13 \\
\hline $\begin{array}{l}\text { STP influent } \\
\text { (ng/l) }\end{array}$ & $\begin{array}{r}16 \\
220 \\
64,42\end{array}$ & $\begin{array}{r}\text { nd } \\
42 \\
11,32\end{array}$ & $\begin{array}{r}31 \\
270 \\
84,50\end{array}$ & $\begin{array}{r}27 \\
1700 \\
446,33\end{array}$ & $\begin{array}{r}150 \\
2600 \\
766,67\end{array}$ & $\begin{array}{r}\text { nd } \\
1400 \\
410\end{array}$ & $\begin{array}{r}14 \\
110 \\
28,08\end{array}$ & 12 \\
\hline
\end{tabular}




\begin{tabular}{|c|c|c|c|c|c|c|c|c|}
\hline & $\begin{array}{r}\text { Alfuzosin } \\
(81403-80-7\end{array}$ & $\begin{array}{r}\text { Cilazapril } \\
(88768-40-5)\end{array}$ & $\begin{array}{r}\text { Diltiazem } \\
(42399-41-7)\end{array}$ & $\begin{array}{r}\text { Eprosartan } \\
(133040-01-4)\end{array}$ & $\begin{array}{r}\text { Irbesartan } \\
(138402-11-6)\end{array}$ & $\begin{array}{r}\text { Telmisartan } \\
(144701-48-4)\end{array}$ & $\begin{array}{r}\text { Verapamil } \\
(52-53-9)\end{array}$ & \\
\hline $\begin{array}{l}\text { Surface water } \\
(\mathrm{ng} / \mathrm{l})\end{array}$ & $\begin{array}{r}0,45 \\
33 \\
7,98\end{array}$ & $\begin{array}{r}\text { nd } \\
4 \\
2,75\end{array}$ & $\begin{array}{r}\text { nd } \\
20 \\
6,12\end{array}$ & $\begin{array}{r}\text { nd } \\
35 \\
13,74\end{array}$ & $\begin{array}{r}2 \\
430 \\
62,10\end{array}$ & $\begin{array}{r}\text { nd } \\
110 \\
110\end{array}$ & $\begin{array}{r}\text { nd } \\
20 \\
19,50\end{array}$ & 10 \\
\hline $\begin{array}{l}\text { Drinking } \\
\text { water (ng/l) }\end{array}$ & nd & nd & $\begin{array}{r}\text { nd } \\
7,90 \\
7,90\end{array}$ & $\begin{array}{r}\text { nd } \\
5,10 \\
5,10\end{array}$ & $\begin{array}{r}\text { nd } \\
2,80 \\
1,65\end{array}$ & nd & nd & 6 \\
\hline
\end{tabular}

\section{Reference:}

- (Fick, et al., 2011)

\begin{tabular}{|c|c|c|}
\hline & Propanolol (525-66-6) & \\
\hline \multirow[t]{3}{*}{ Matrix } & $\min$ & Nr.S \\
\hline & $\max$ & \\
\hline & $A D$ & \\
\hline Biota & nd & 3 \\
\hline Sediment & nd & 6 \\
\hline \multirow[t]{3}{*}{ STP sludge (ng/g dw) } & 12,3 & 4 \\
\hline & 30,3 & \\
\hline & 19,6 & \\
\hline \multirow[t]{3}{*}{ Effluent (ng/l) } & nd & 10 \\
\hline & 77,7 & \\
\hline & 39,9 & \\
\hline \multirow[t]{3}{*}{ Surface water (ng/l) } & nd & 12 \\
\hline & 3 & \\
\hline & 1,66 & \\
\hline
\end{tabular}

\section{Reference:}

- (Schlabach, et al., 2009) 



\section{Complexing compounds}

Complexing forms are defined as chelant compounds, which form complexes with calcium, magnesium, and iron, allowing better foaming and cleaning performance of cosmetics and personal care products. By binding with metal ions, these ingredients prevent the metals from deposition on hair, scalp and skin.

Chelante compounds are heterocyclic chemicals with a central metallic ion attached by covalent bonds to two or more non-metallic atoms in the same molecule.

Disodium EDTA and the related ingredients bind to metal ions which inactivates them. The binding of metal ions helps prevent the deterioration of cosmetics and personal care products. It also helps to maintain clarity, protect fragrance compounds, and prevent rancidity.

Ethylenediaminetetraacetic acid (EDTA) is the only complexing compound that has been monitored in the Nordic countries.

\subsubsection{Ethylenediaminetetraacetic acid (EDTA)}

CAS nr.: 60-00-4

Info: Ligand and chelating agent, i.e. its ability to "sequester" metal ions Toxicity data: LD50 (Lepomis macrochirus) $=24000000 \mathrm{ng} / \mathrm{L}$

(Schlabach, et al., 2009)

PNECaqua $=2,2$ mg/l (Schlabach, et al., 2009)

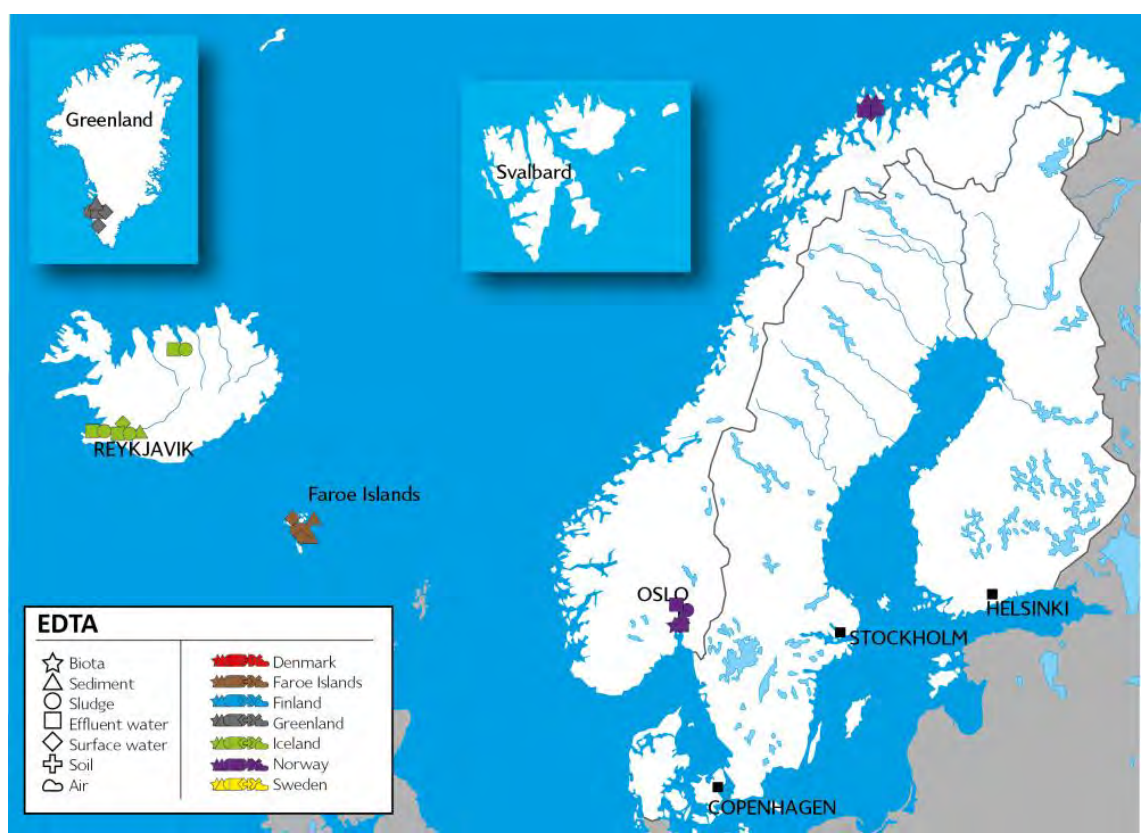



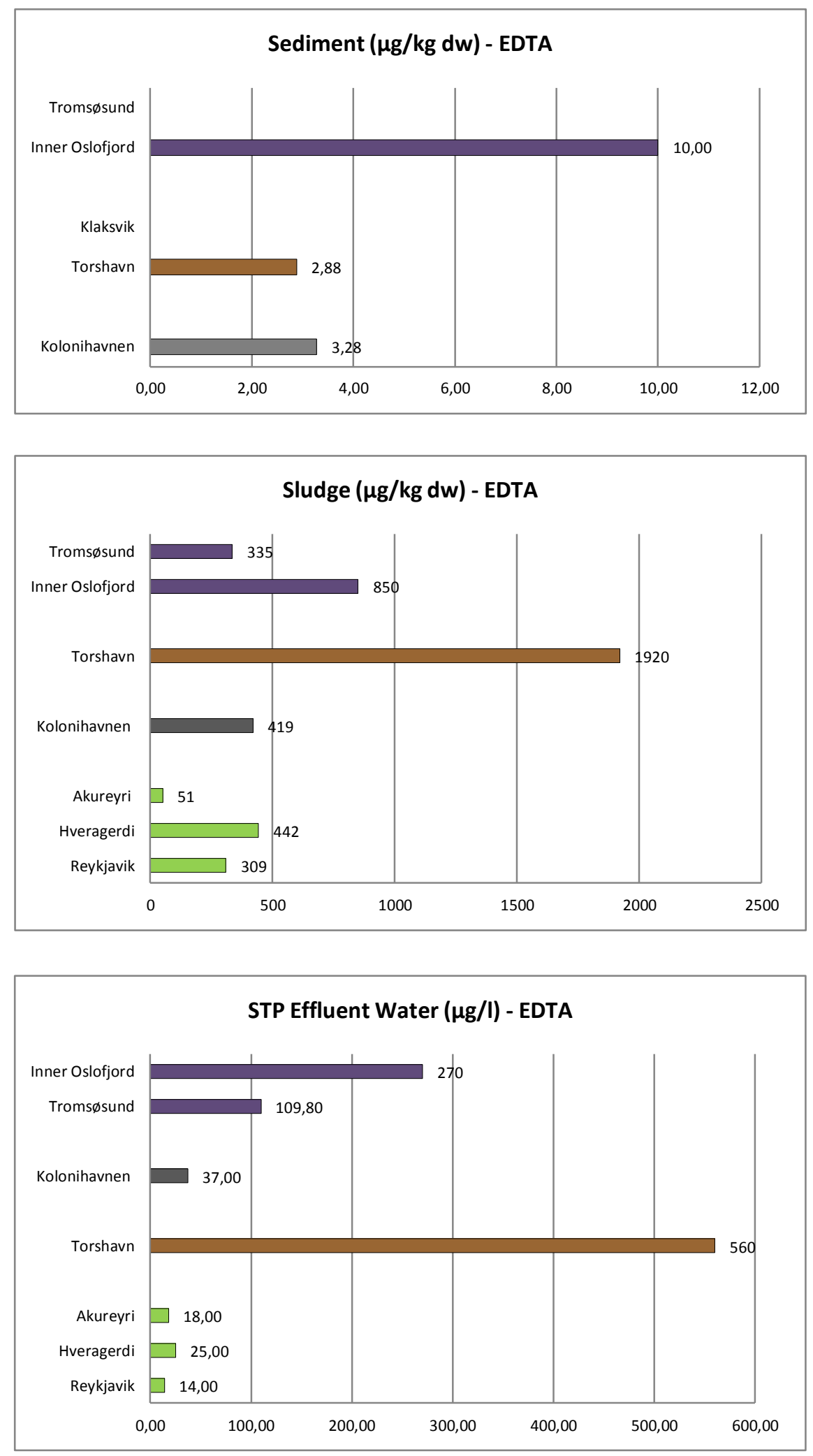


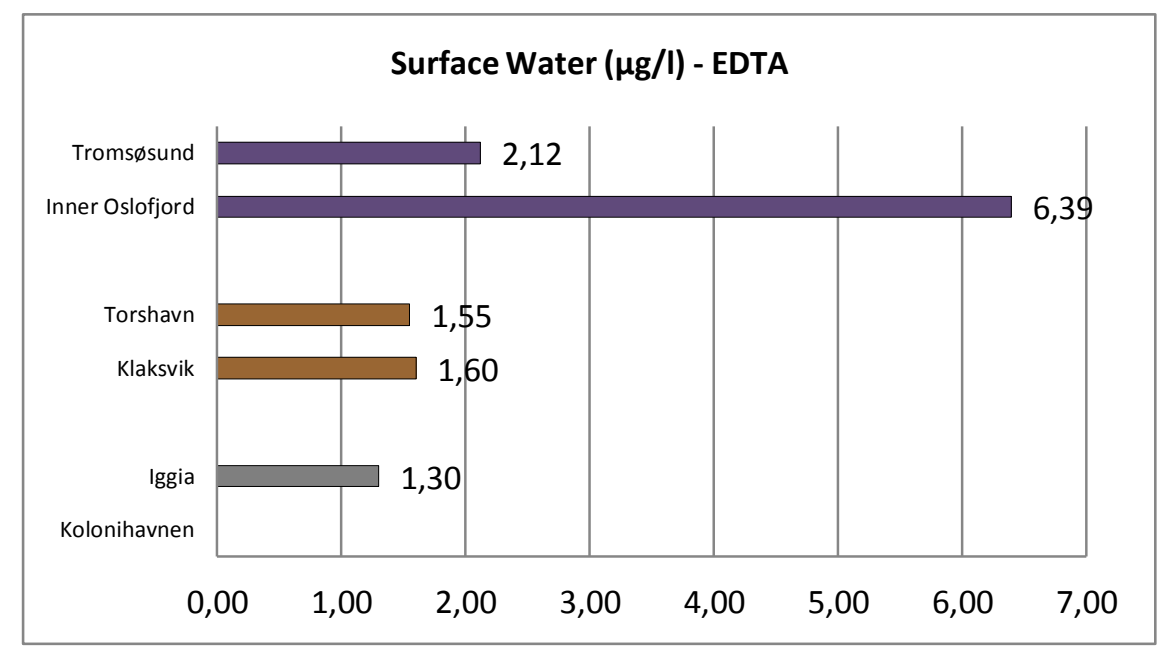

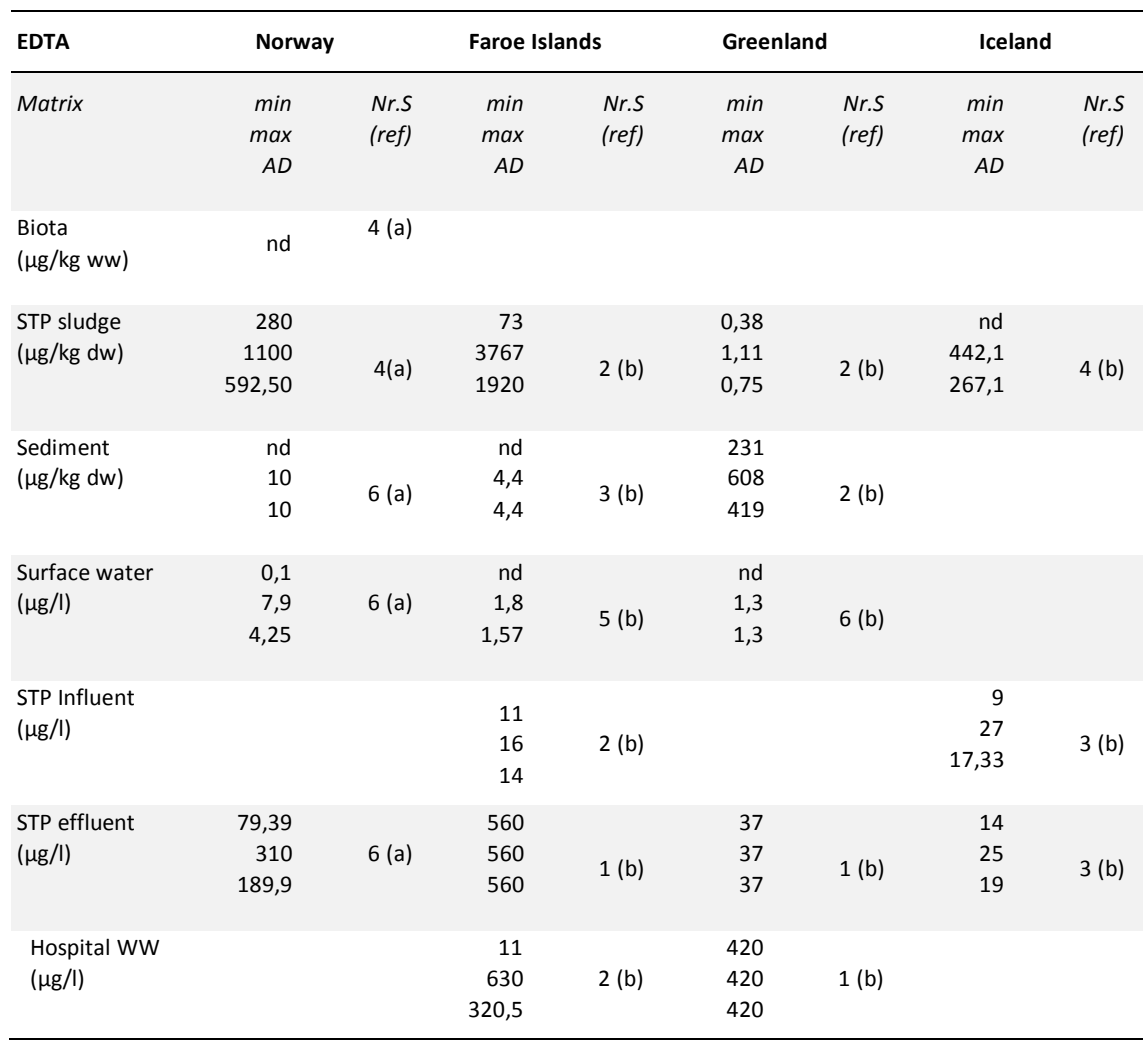

\section{References:}

a) (Schlabach, et al., 2007)

b) (Huber, et al., NA)

\section{Conclusion:}

The compound was monitored in Norway, Faroe Islands, Greenland and Iceland. It was detected in several matrices: 
- STP sludge in all countries and all samples analysed. The values detected were high for all countries. The highest average detected value is observed in the Faroe Islands

- Sediment in Faroe Islands and Greenland, not in Norway.

- Surface water in Faroe Islands, Greenland and Norway, in the large majority of samples monitored. Higher values were detected in Norway. All values detected were below the PNEC

- STP effluent in all countries. Values detected in Faroe Islands were higher. All values detected were below the PNEC

- Hospital waste water in all countries monitored, in values below the PNEC. Lower values were observed in Greenland

- STP influent in all countries monitored, values detected were below the PNEC 


\section{Fragrances}

\subsection{Scope and definition}

Fragrances are defined as compounds that participate in giving a "pleasant scent" to a product.

The compounds listed in Table 5 are fragrances that have been monitored in at least one Nordic country.

\begin{tabular}{ll} 
Table 5: Fragrances identified in monitoring studies in the Nordic countries & \\
\hline Compounds monitored & CAS nr \\
\hline d-limonene, & $5989-27-5$ \\
I-limonene, & $5989-54-8$ \\
Ethylenebrassylat & $105-95-3$ \\
Cashmeran, 6,7-Dihydro-1,1,2,3,3-pentamethyl-4(5H)indanone & $33704-61-9$ \\
Musk moskene, 4,6-dinitro-1,1,3,3,5-pentamethylindane & $116-66-5$ \\
Phantolide, 6-Acetyl-1,1,2,3,3,5-hexamethyldihydroindene & $15323-35-0$ \\
Musk xylene, 5-tert-butyl-2,4,5-trinitro-m-xylene & $81-15-2$ \\
Musk tibetene, 1-tert-butyl-2,6-dinitro-3,4,5-trimethylbenzene & $145-39-1$ \\
Traseolide, 5-Acetyl-1,1,2,6-tetramethyl-3-isopropyl-dihydroindene & $68140-48-7$ \\
Tonalide, 7-Acetyl-1,1,3,4,4,6-hexamethyltetrahydro-naphtlene & $1506-02-1$ \\
Galaxolide, 1,3,4,6,7,8-Hexahydro-4,6,6,7,8,8-hexamethylcyclopenta[g]-2-benzopyrane & $1222-05-5$ \\
Celestolide, 4-Acetyl-1,1-dimethyl-6-tertbutyldihydroindene & $13171-00-1$ \\
Musk ambrette, 1-tert-Butyl-2-methoxy-4-methyl-3,5-dinitrobenzene & $83-66-9$ \\
Musk ketone, 1-tert-Butyl-3,5-dimethyl-2,6-dinitrobenzene & $81-14-1$
\end{tabular}

\subsection{Compounds mapped}

\subsubsection{Musk ketone}

CAS nr.: 81-14-1

Info: Used as fragrances in soaps, perfumes, air fresheners, detergents, fabric softeners and other household cleaning products

Toxicity data:

Minimum NOEC and LOEC $=0,010 \mathrm{mg} / \mathrm{l}$

(Törneman, 2008)

PNEC =

PNEC aqua $=1 \mu \mathrm{g} / \mathrm{l}$

PNEC sediment $=220 \mu \mathrm{g} / \mathrm{kg}$

(Törneman, 2008) 

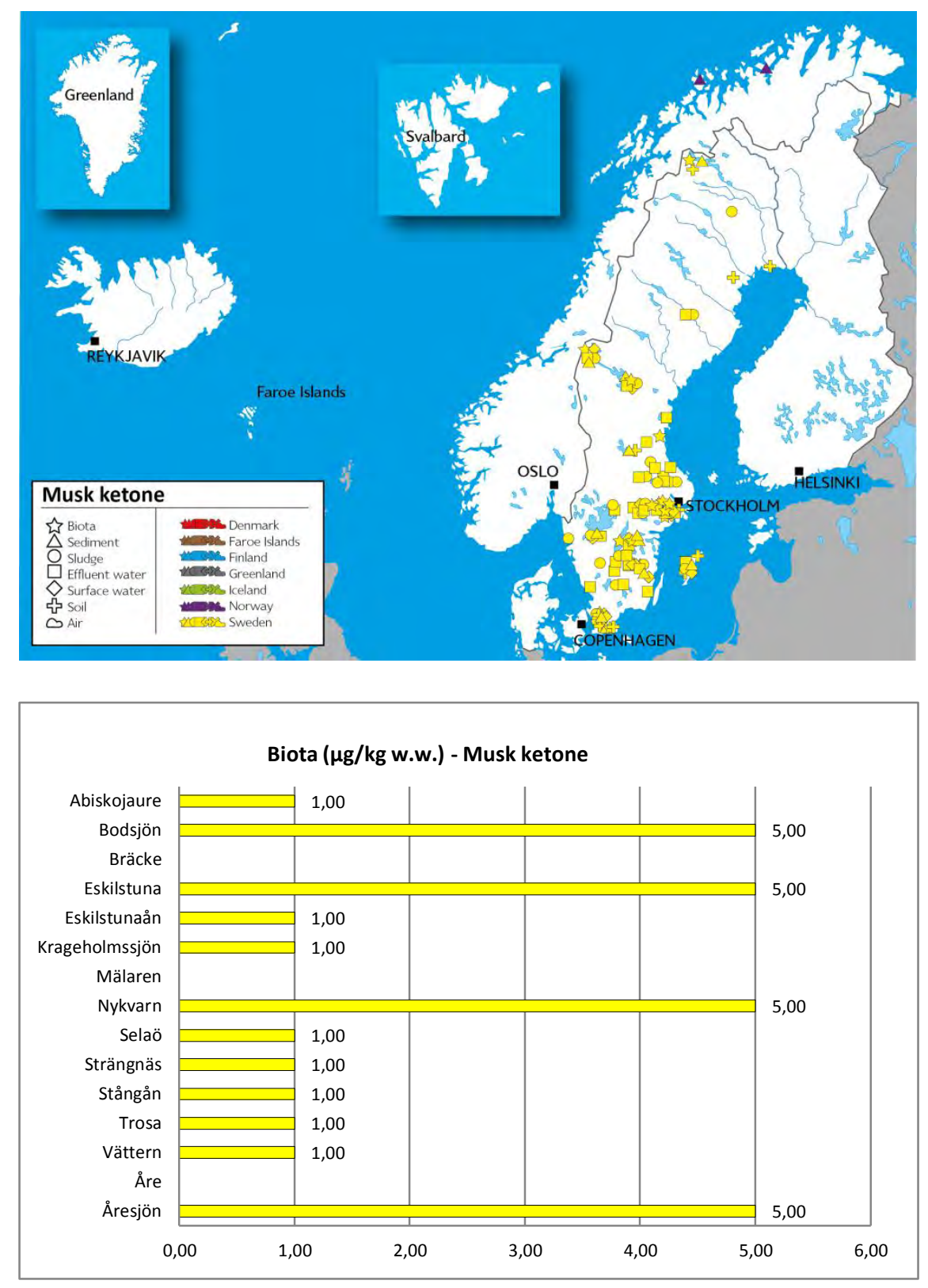


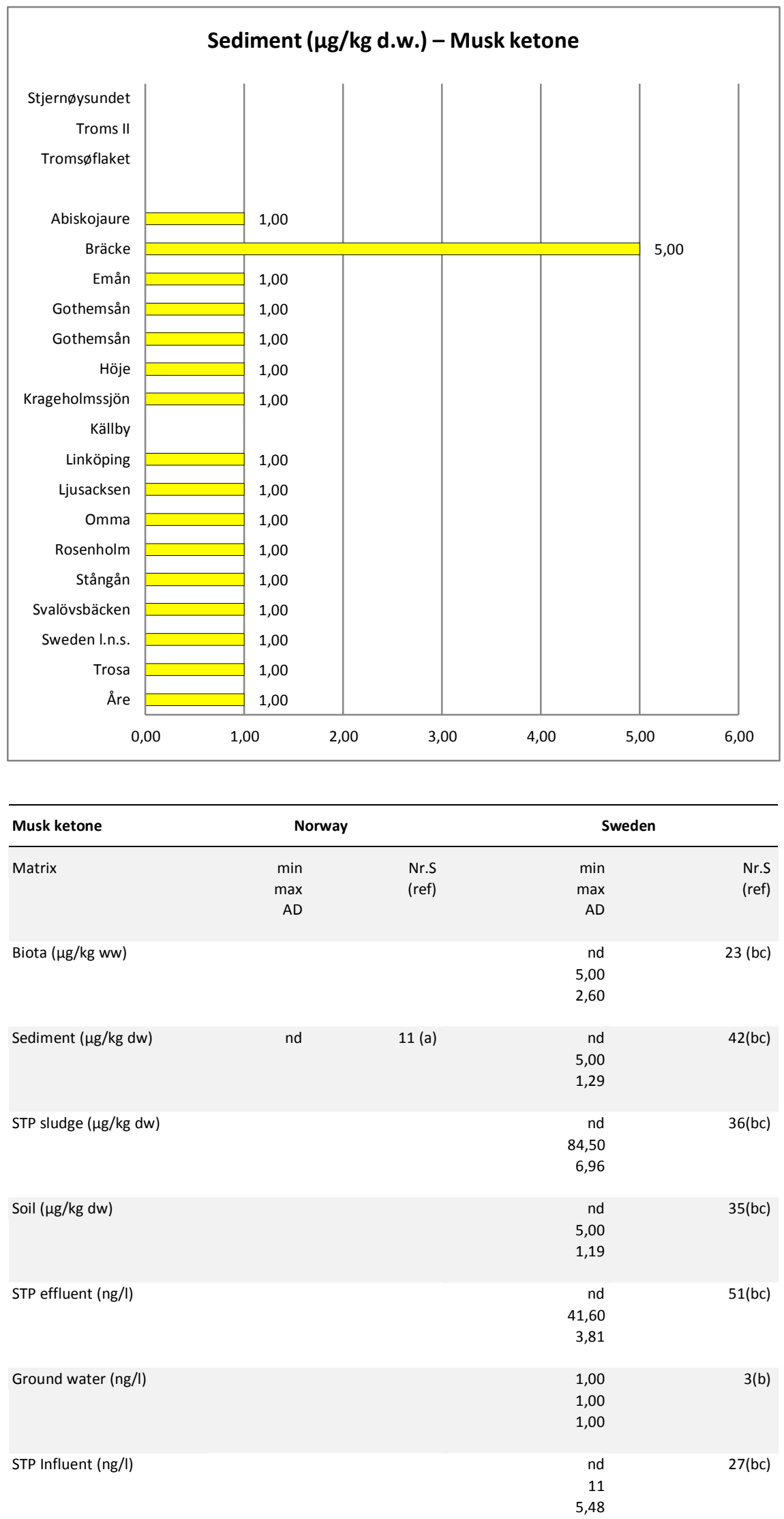




\begin{tabular}{|c|c|c|c|}
\hline Musk ketone & Norway & \multicolumn{2}{|c|}{ Sweden } \\
\hline \multirow[t]{3}{*}{ Leachate (ng/l) } & & 1,00 & $4(\mathrm{bc})$ \\
\hline & & 5,00 & \\
\hline & & 2,33 & \\
\hline \multirow[t]{3}{*}{ Storm water (ng/l) } & & 1,00 & $4(b c)$ \\
\hline & & 1,00 & \\
\hline & & 1,00 & \\
\hline \multirow[t]{3}{*}{ Surface water (ng/l) } & & 1,00 & $34(b c)$ \\
\hline & & 3,7 & \\
\hline & & 1,37 & \\
\hline
\end{tabular}

\section{References:}

a) (Bakke, et al., 2008)

b) (Törneman, 2008)

c) (Törneman, 2010)

\section{Conclusion:}

The compound was monitored in throughout Sweden and in Norway. It was not detected in Sediment in Norway, but was detected in all matrices monitored in Sweden:

- Biota, in almost all locations monitored, the results varied.

- Sediment, in almost all locations monitored,in values below the PNEC

- Soil in all locations monitored, one higher value was detected in one location

- Surface water, in almost all locations monitored, below the PNEC

- STP sludge, in all locations monitored

- STP effluent, in all locations monitored, below the PNEC

\subsubsection{Musk xylene}

CAS nr.: 81-15-2

Info: was the most widely used of the "nitro-musks", a synthetic musk fragrance, which mimics natural musk. It has been used as a perfume fixative in a wide variety of consumer products, and is still used in some cosmetics and fragrances

Toxicity data: NOEC and LOEC $\min 0,056 \mathrm{mg} / \mathrm{l}$

(Törneman, 2008)

$P N E C=$

- PNEC aqua $1,1 \mu \mathrm{g} / \mathrm{l}$

- PNEC sediment $300 \mu \mathrm{g} / \mathrm{kg}$ "

- PNEC soil $260 \mu \mathrm{g} / \mathrm{kg}$

(Törneman, 2008) 

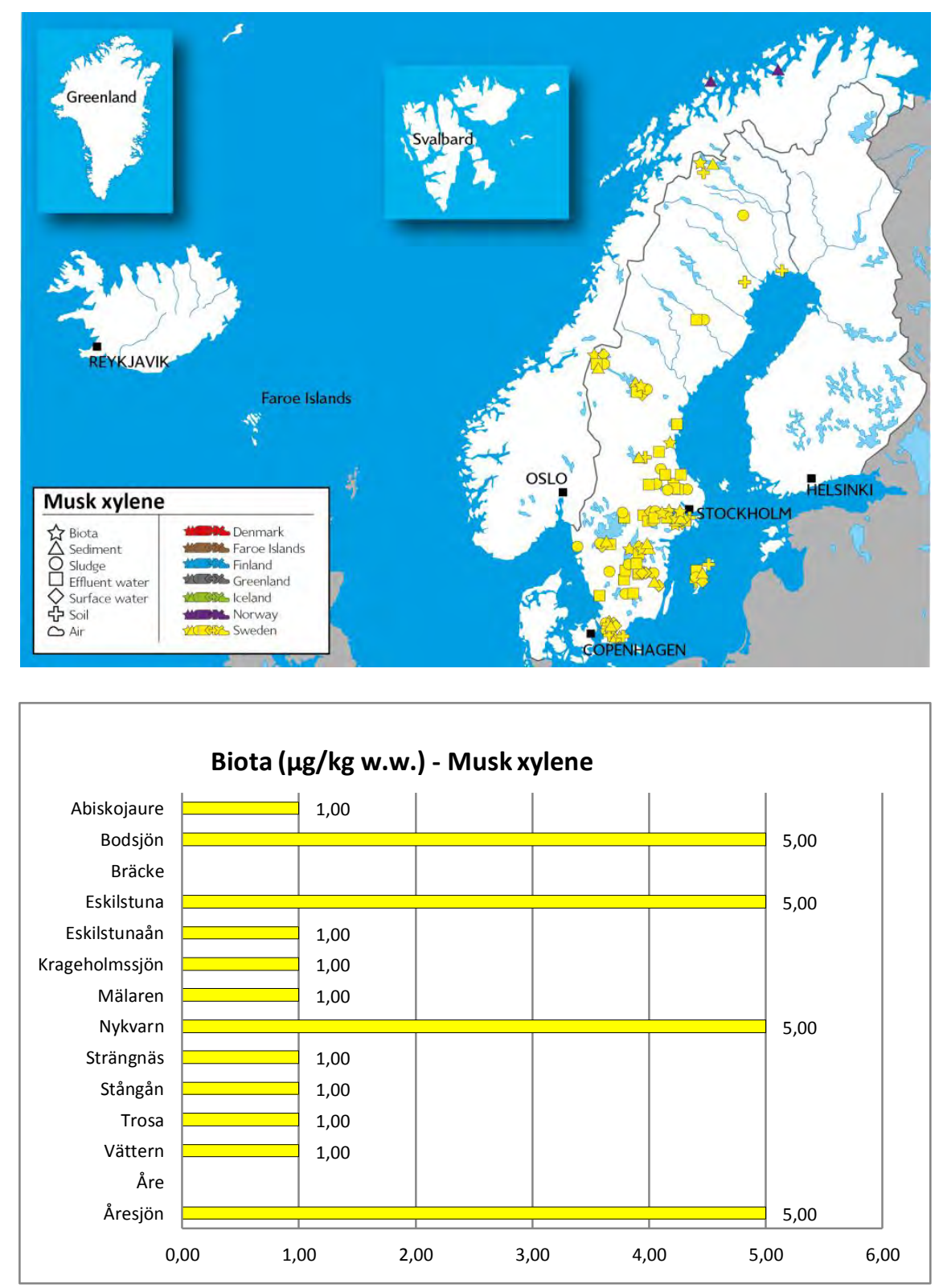


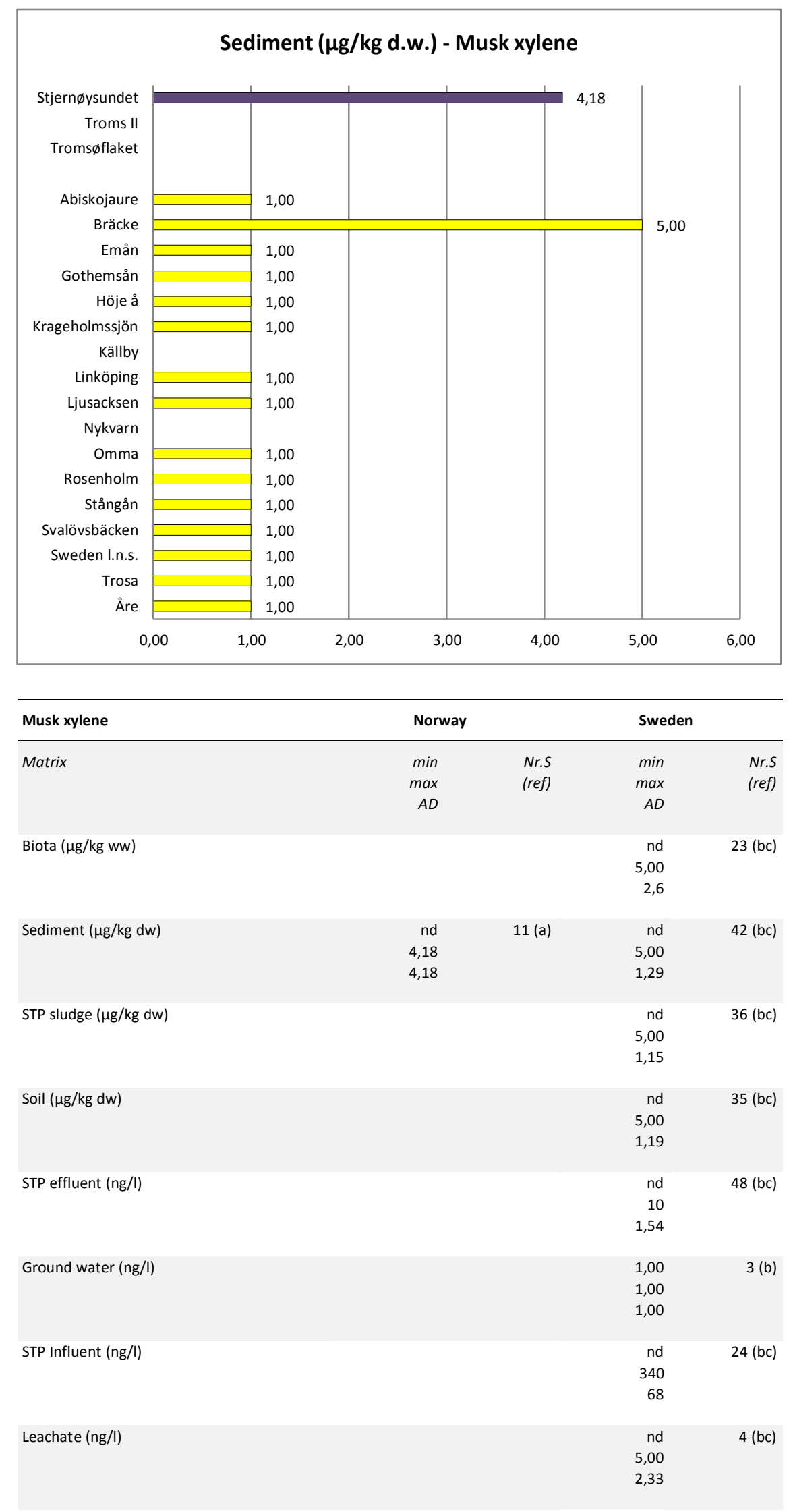




\begin{tabular}{|c|c|c|c|}
\hline \multirow{2}{*}{$\begin{array}{l}\text { Musk xylene } \\
\text { Storm water (ng/l) }\end{array}$} & \multirow[t]{2}{*}{ Norway } & \multicolumn{2}{|c|}{ Sweden } \\
\hline & & 1,00 & $4(b)$ \\
\hline & & 1,00 & \\
\hline & & 1,00 & \\
\hline \multirow[t]{3}{*}{ Surface water (ng/l) } & & nd & $34(b c)$ \\
\hline & & 8,70 & \\
\hline & & 2,29 & \\
\hline
\end{tabular}

\section{References:}

a) (Bakke, et al., 2008)

b) (Törneman, 2008)

c) (Törneman, 2010)

\section{Conclusion:}

The compound was monitored in Norway and Sweden. It was not detected in sediment in Norway, but was detected in all matrices monitored in Sweden:

- Biota, in almost all locations monitored

- Sediment, in almost all locations monitored, below the PNEC

- Soil, in all locations monitored, below the PNEC

- Surface water, in all locations monitored, below the PNEC

- STP sludge, in all locations monitored

- STP effluent, in all locations monitored, below the PNEC

\subsubsection{Cashmeran}

CAS nr.: 33704-61-9

Info: Synthetic fragrance in cosmetic, known to bioaccumulateand be a potential hormone disruptor.

Toxicity data: LD50 oral $=2900 \mathrm{mg} / \mathrm{kg}$

(Törneman, 2008)

$P N E C=\mathrm{NA}$ 

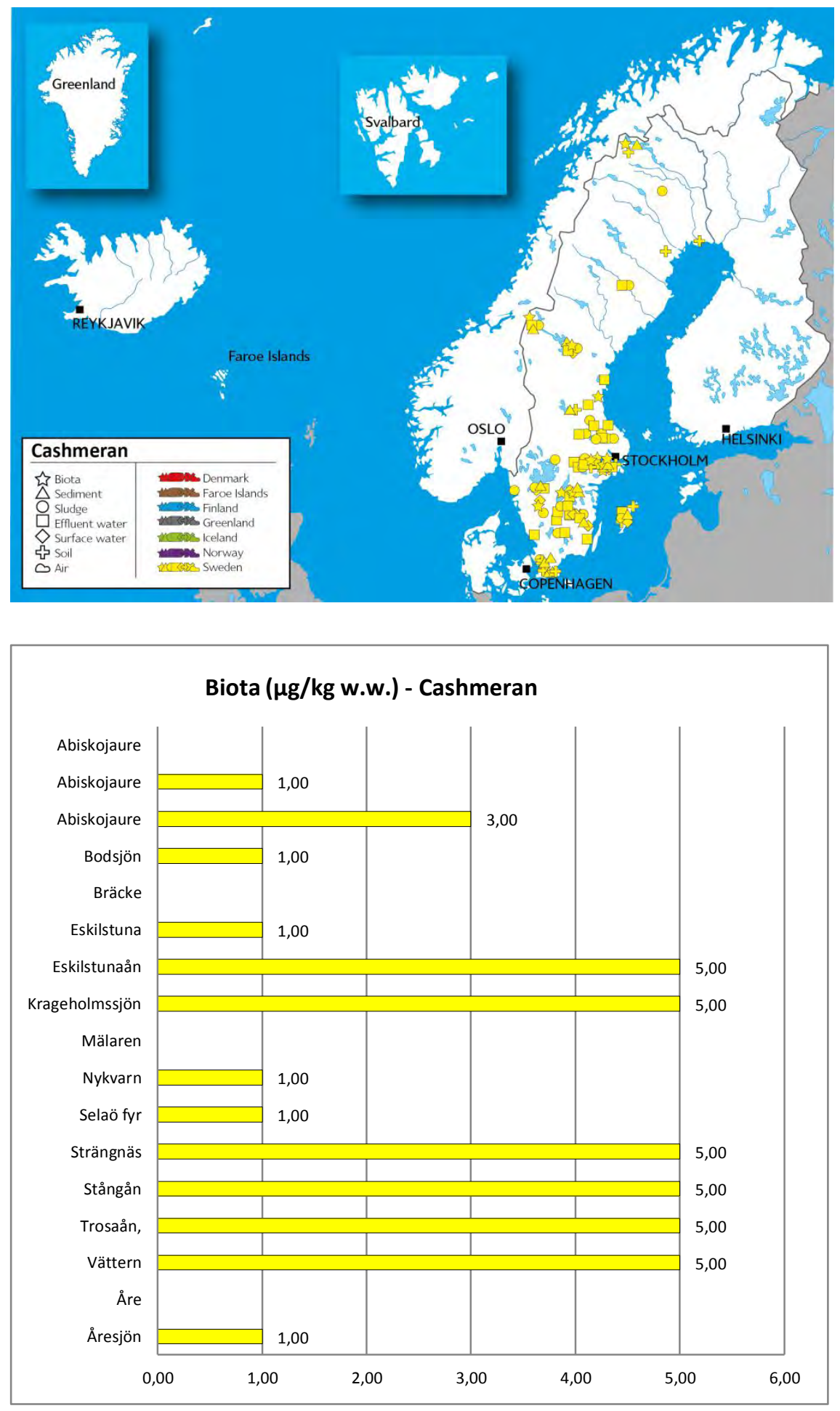


\begin{tabular}{|c|c|c|}
\hline Cashmeran & Sweden & \\
\hline \multirow[t]{3}{*}{ Matrix } & $\min$ & Nr.S \\
\hline & $\max$ & (ref) \\
\hline & $A D$ & \\
\hline \multirow[t]{3}{*}{ Biota $(\mu \mathrm{g} / \mathrm{kg} w w)$} & nd & $23(a b)$ \\
\hline & 5,00 & \\
\hline & 2,87 & \\
\hline \multirow[t]{3}{*}{ Sediment $(\mu \mathrm{g} / \mathrm{kg} \mathrm{dw})$} & 1,00 & $42(a b)$ \\
\hline & 12,20 & \\
\hline & 2,01 & \\
\hline \multirow[t]{3}{*}{ STP sludge $(\mu \mathrm{g} / \mathrm{kg} \mathrm{dw})$} & nd & $36(a b)$ \\
\hline & 2510 & \\
\hline & 119,94 & \\
\hline \multirow[t]{3}{*}{ Soil ( $\mu g / k g d w)$} & nd & $35(a b)$ \\
\hline & 20,00 & \\
\hline & 2,76 & \\
\hline \multirow[t]{3}{*}{ Influent (ng/l) } & 1,00 & $27(a b)$ \\
\hline & 150,00 & \\
\hline & 53,81 & \\
\hline \multirow[t]{3}{*}{ STP effluent (ng/l) } & 1,00 & $51(a b)$ \\
\hline & 308,00 & \\
\hline & 19,11 & \\
\hline \multirow[t]{3}{*}{ Groundwater (ng/l) } & 1,00 & $3(a)$ \\
\hline & 1,00 & \\
\hline & 1,00 & \\
\hline \multirow[t]{3}{*}{ Surface water (ng/l) } & 1,00 & $4(a b)$ \\
\hline & 1,00 & \\
\hline & 1,00 & \\
\hline \multirow[t]{3}{*}{ Surface water(ng/l) } & nd & $34(a b)$ \\
\hline & 26,00 & \\
\hline & 6,21 & \\
\hline \multirow[t]{3}{*}{ Leachate (ng/l) } & nd & $4(a b)$ \\
\hline & 5,00 & \\
\hline & 2,33 & \\
\hline \multirow[t]{3}{*}{ Storm water (ng/l) } & 1,00 & $4(a)$ \\
\hline & 1,00 & \\
\hline & 1,00 & \\
\hline
\end{tabular}

\section{References:}

a) (Törneman, 2008)

b) (Törneman, 2010)

\section{Conclusion:}

The compound was only monitored in Sweden and was detected in all locations monitored. 


\subsubsection{Celestolide}

CAS nr.: 13171-00-1

Info: Celestolide is a polycyclic musk commonly used as a fragrance ingredient in a wide variety of personal care and other everyday products, including cosmetics, cleaning agents, detergents, air fresheners, and perfumes

Toxicity data: LD50 oral $=2900 \mathrm{mg} / \mathrm{kg}$

(Törneman, 2008)

$P N E C=\mathrm{NA}$
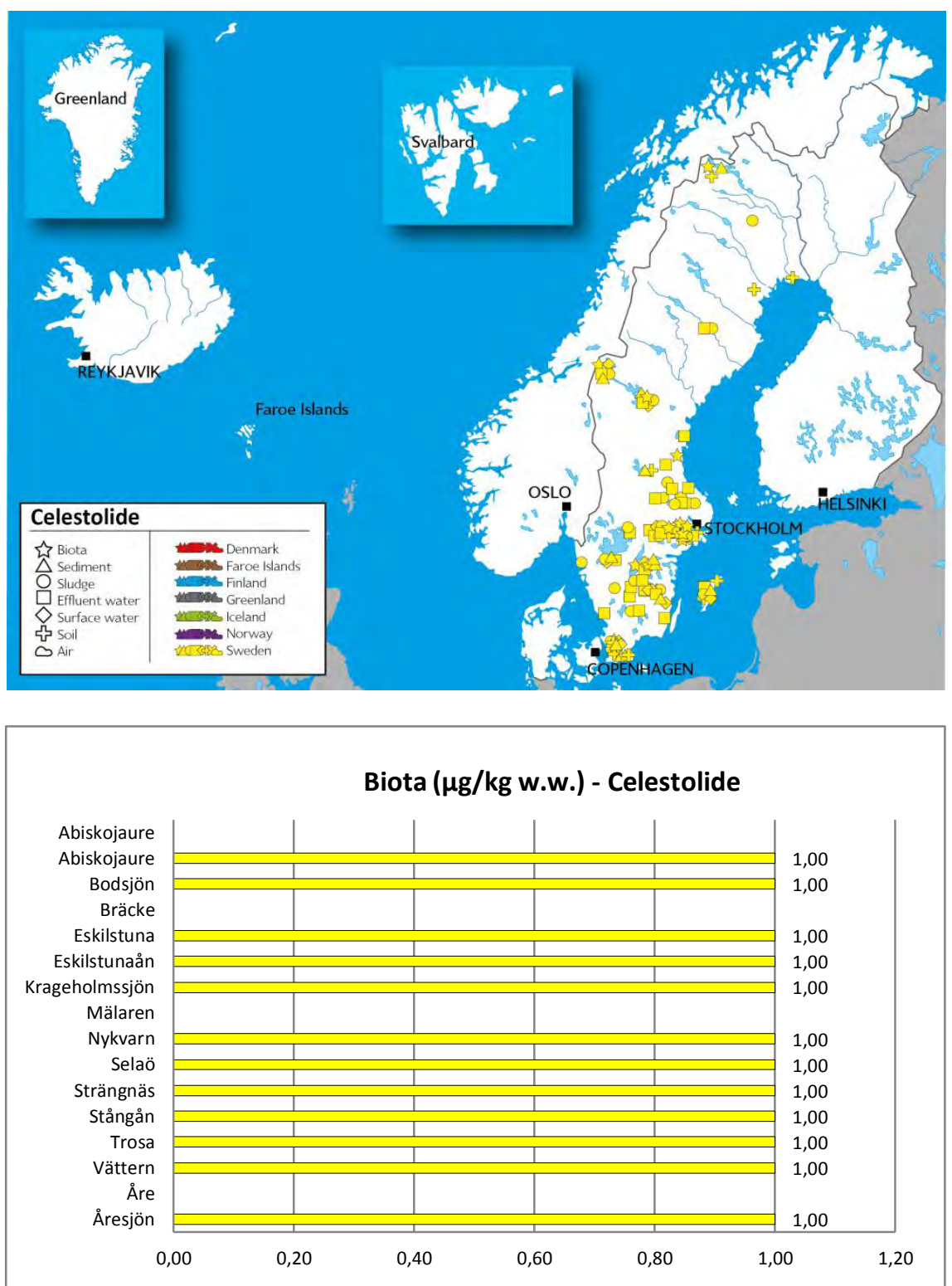


\begin{tabular}{|c|c|c|}
\hline \multirow{2}{*}{$\begin{array}{l}\text { Celestolide } \\
\text { Matrix }\end{array}$} & \multicolumn{2}{|c|}{ Sweden } \\
\hline & $\min$ & Nr.S \\
\hline & $\max$ & (ref) \\
\hline & $A D$ & \\
\hline \multirow[t]{3}{*}{ Biota $(\mu \mathrm{g} / \mathrm{kg} w w)$} & nd & $23(a b)$ \\
\hline & 1,00 & \\
\hline & 1,00 & \\
\hline \multirow[t]{3}{*}{ Sediment $(\mu \mathrm{g} / \mathrm{kg} \mathrm{dw})$} & nd & $42(a b)$ \\
\hline & 5,00 & \\
\hline & 1,28 & \\
\hline \multirow[t]{3}{*}{ STP sludge $(\mu \mathrm{g} / \mathrm{kg} \mathrm{dw})$} & nd & $36(a b)$ \\
\hline & 124,00 & \\
\hline & 23,22 & \\
\hline \multirow[t]{3}{*}{ Soil ( $\mu \mathrm{g} / \mathrm{kg} \mathrm{dw})$} & nd & 28 \\
\hline & 5,10 & \\
\hline & 1,55 & \\
\hline \multirow[t]{3}{*}{ Influent (ng/l) } & nd & $27(a b)$ \\
\hline & 17,00 & \\
\hline & 5,48 & \\
\hline \multirow[t]{3}{*}{ STP effluent (ng/l) } & nd & $52(a b)$ \\
\hline & 4,00 & \\
\hline & 1,47 & \\
\hline \multirow[t]{3}{*}{ Groundwater (ng/l) } & 1,00 & $3(a b)$ \\
\hline & 1,00 & \\
\hline & 1,00 & \\
\hline \multirow[t]{3}{*}{ Surface water (ng/l) } & nd & $33(a b)$ \\
\hline & 3,00 & \\
\hline & 1,15 & \\
\hline \multirow[t]{3}{*}{ Leachate (ng/l) } & nd & $4(a b)$ \\
\hline & 5,00 & \\
\hline & 2,33 & \\
\hline \multirow[t]{3}{*}{ Storm water (ng/l) } & 1,00 & 4 (a) \\
\hline & 1,00 & \\
\hline & 1,00 & \\
\hline
\end{tabular}

References:

a) (Törneman, 2008)

b) (Törneman, 2010)

\section{Conclusion:}

The compound was only monitored in Sweden, however it was monitored in two studies in biota and in this manner the compound was mapped and graphed for biota. The compound was detected in all matrices sampled and in all locations monitored. 


\subsubsection{Galaxolide}

CAS nr.: 80450-66-4

Info: polycyclic musk commonly used as a fragrance ingredient in a wide variety of personal care and other everyday products, including cosmetics, cleaning agents, detergents, air fresheners, and perfumes

Toxicity data: NOEC and LOEC $\max =0,14 \mathrm{mg} / \mathrm{l}$

(Törneman, 2008)

$P N E C=$

PNEC aqua $=6,8 \mu \mathrm{g} / \mathrm{l}$

PNEC sediment $=320 \mu \mathrm{g} / \mathrm{l}$

(Törneman, 2008)
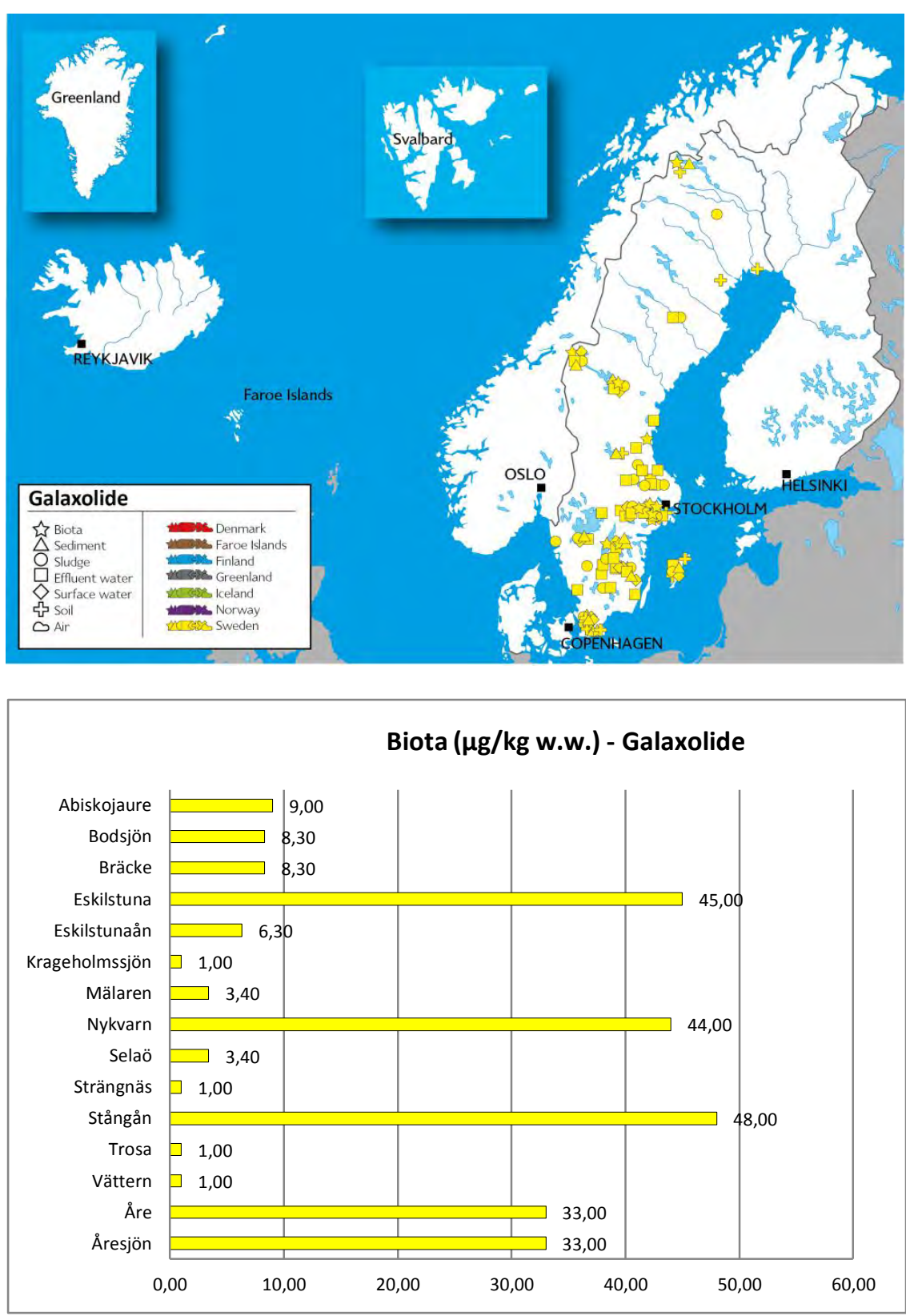


\begin{tabular}{|c|c|c|}
\hline \multirow{2}{*}{$\begin{array}{l}\text { Galaxolide } \\
\text { Matrix }\end{array}$} & \multicolumn{2}{|c|}{ Sweden } \\
\hline & $\min$ & Nr.S \\
\hline & $\max$ & (ref) \\
\hline & $A D$ & \\
\hline Biota & 1,00 & $23(a b)$ \\
\hline \multirow[t]{2}{*}{$(\mu \mathrm{g} / \mathrm{kg} w w)$} & 48,00 & \\
\hline & 18,09 & \\
\hline Sediment ( & nd & $42(a b)$ \\
\hline \multirow[t]{2}{*}{$\mu \mathrm{g} / \mathrm{kg} \mathrm{dw})$} & 100 & \\
\hline & 18,12 & \\
\hline \multirow[t]{3}{*}{ STP sludge $(\mu \mathrm{g} / \mathrm{kg} \mathrm{dw})$} & 8,70 & $36(a b)$ \\
\hline & 29400 & \\
\hline & 6116,91 & \\
\hline \multirow[t]{3}{*}{ Soil ( $\mu \mathrm{g} / \mathrm{kg} \mathrm{dw})$} & nd & $35(a b)$ \\
\hline & 870 & \\
\hline & 68,01 & \\
\hline \multirow[t]{3}{*}{ Influent (ng/l) } & 53,70 & $27(a b)$ \\
\hline & 3800 & \\
\hline & 1469,93 & \\
\hline \multirow[t]{3}{*}{ STP effluent (ng/l) } & 1,00 & $51(a b)$ \\
\hline & 20300 & \\
\hline & 891,71 & \\
\hline \multirow[t]{3}{*}{ Groundwater (ng/l) } & 1,00 & 3 (a) \\
\hline & 1,00 & \\
\hline & 1,00 & \\
\hline \multirow[t]{3}{*}{ Surface water (ng/l) } & nd & $34(a b)$ \\
\hline & 800 & \\
\hline & 109,76 & \\
\hline \multirow[t]{3}{*}{ Leachate (ng/l) } & 1,00 & $4(a b)$ \\
\hline & 16,00 & \\
\hline & 8,50 & \\
\hline \multirow[t]{3}{*}{ Storm water (ng/l) } & 1,00 & 4 (a) \\
\hline & 1,00 & \\
\hline & 1,00 & \\
\hline
\end{tabular}

References:

a) (Törneman, 2008)

b) (Törneman, 2010)

\section{Conclusion:}

The compound was only monitored in Sweden and was detected in all locations monitored. High values were detected in STP sludge and effluent. In sediment it was detected in values below the PNEC. High values were detected in STP sludge and effluent, the values detcetd in effluent were above the PNEC. The values detected in surface water were below the PNEC. 


\subsubsection{Musk ambrette}

CAS nr.: 83-66-9

Info: Musk ambrette is a nitro-musk compound widely used as a fixative in fragrance formulations and found to a lesser degree in flavor compositions

Toxicity data: NA

$P N E C=\mathrm{NA}$
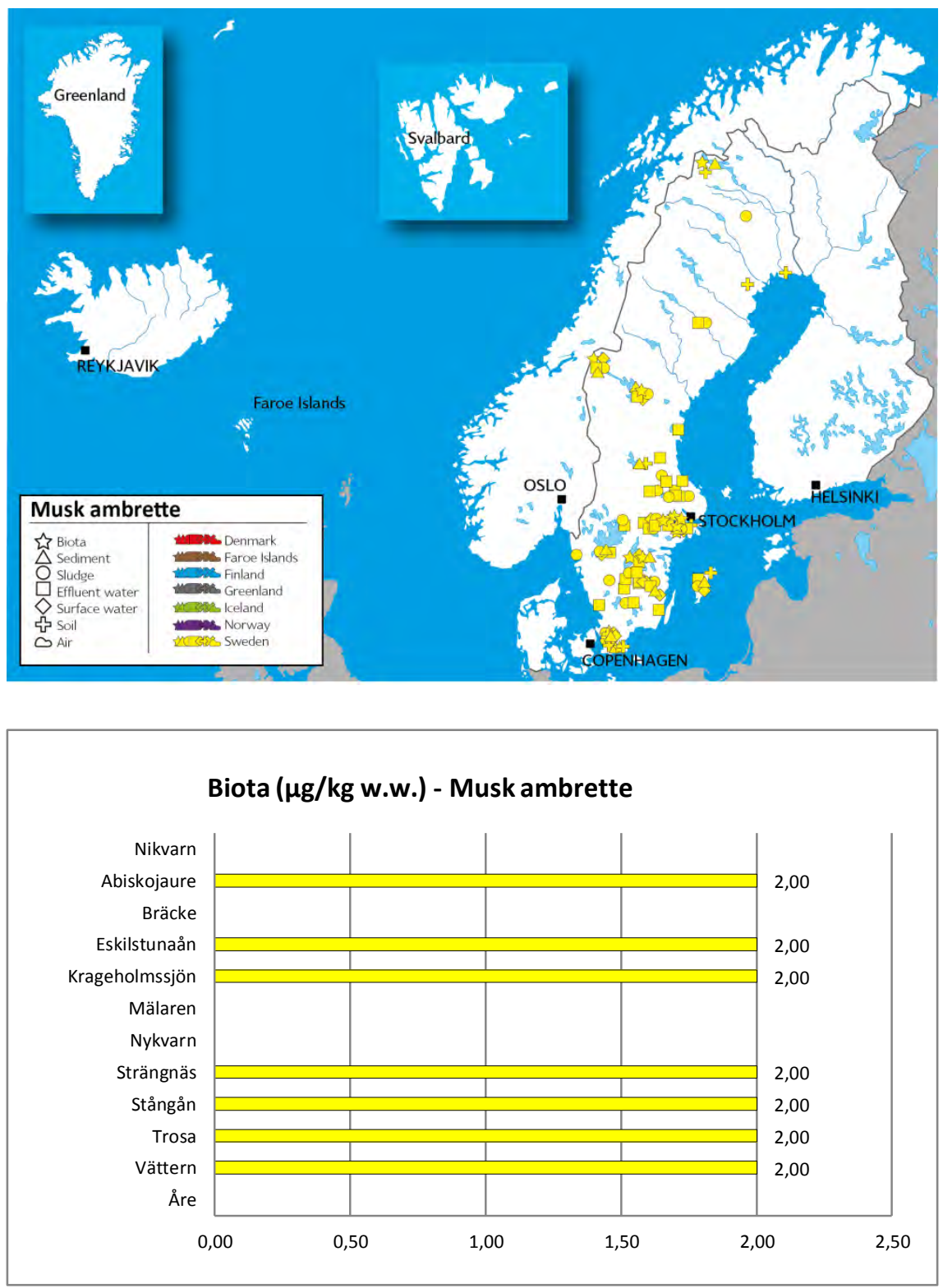


\begin{tabular}{|c|c|c|}
\hline Musk ambrette & & \\
\hline \multirow[t]{3}{*}{ Matrix } & $\min$ & Nr.S \\
\hline & $\max$ & (ref) \\
\hline & $A D$ & \\
\hline \multirow[t]{3}{*}{ Biota $(\mu \mathrm{g} / \mathrm{kg} w w)$} & nd & $23(a b)$ \\
\hline & 2,00 & \\
\hline & 2,00 & \\
\hline \multirow[t]{3}{*}{ Sediment $(\mu \mathrm{g} / \mathrm{kg} \mathrm{dw})$} & nd & $42(a b)$ \\
\hline & 49 & \\
\hline & 5,98 & \\
\hline \multirow[t]{3}{*}{ STP sludge $(\mu \mathrm{g} / \mathrm{kg} \mathrm{dw})$} & nd & $36(a b)$ \\
\hline & 110 & \\
\hline & 16,69 & \\
\hline \multirow[t]{3}{*}{ Soil ( $\mu \mathrm{g} / \mathrm{kg} \mathrm{dw})$} & nd & $35(a b)$ \\
\hline & 23 & \\
\hline & 5,89 & \\
\hline \multirow[t]{3}{*}{ Influent (ng/l) } & nd & $27(a b)$ \\
\hline & 61 & \\
\hline & 14,85 & \\
\hline \multirow[t]{3}{*}{ STP effluent (ng/l) } & nd & $51(a b)$ \\
\hline & 10 & \\
\hline & 1,25 & \\
\hline \multirow[t]{3}{*}{ Groundwater (ng/l) } & 1,00 & 3 (a) \\
\hline & 1,00 & \\
\hline & 1,00 & \\
\hline \multirow[t]{3}{*}{ Surface water (ng/l) } & nd & $34(a b)$ \\
\hline & 3 & \\
\hline & 1,10 & \\
\hline \multirow[t]{3}{*}{ Leachate (ng/l) } & nd & $4(a b)$ \\
\hline & 5 & \\
\hline & 2,33 & \\
\hline \multirow[t]{3}{*}{ Storm water (ng/l) } & 1,00 & $4(a)$ \\
\hline & 1,00 & \\
\hline & 1,00 & \\
\hline
\end{tabular}

References:

a) (Törneman, 2008)

b) (Törneman, 2010)

\section{Conclusion:}

The compound was only monitored in Sweden and was detected in all locations monitored. 


\subsubsection{Musk moskene}

CAS nr.: 116-66-5

Info: Synthetic fragrance in perfumes and cosmetics

Toxicity data: $\mathrm{NA}$

$P N E C=\mathrm{NA}$
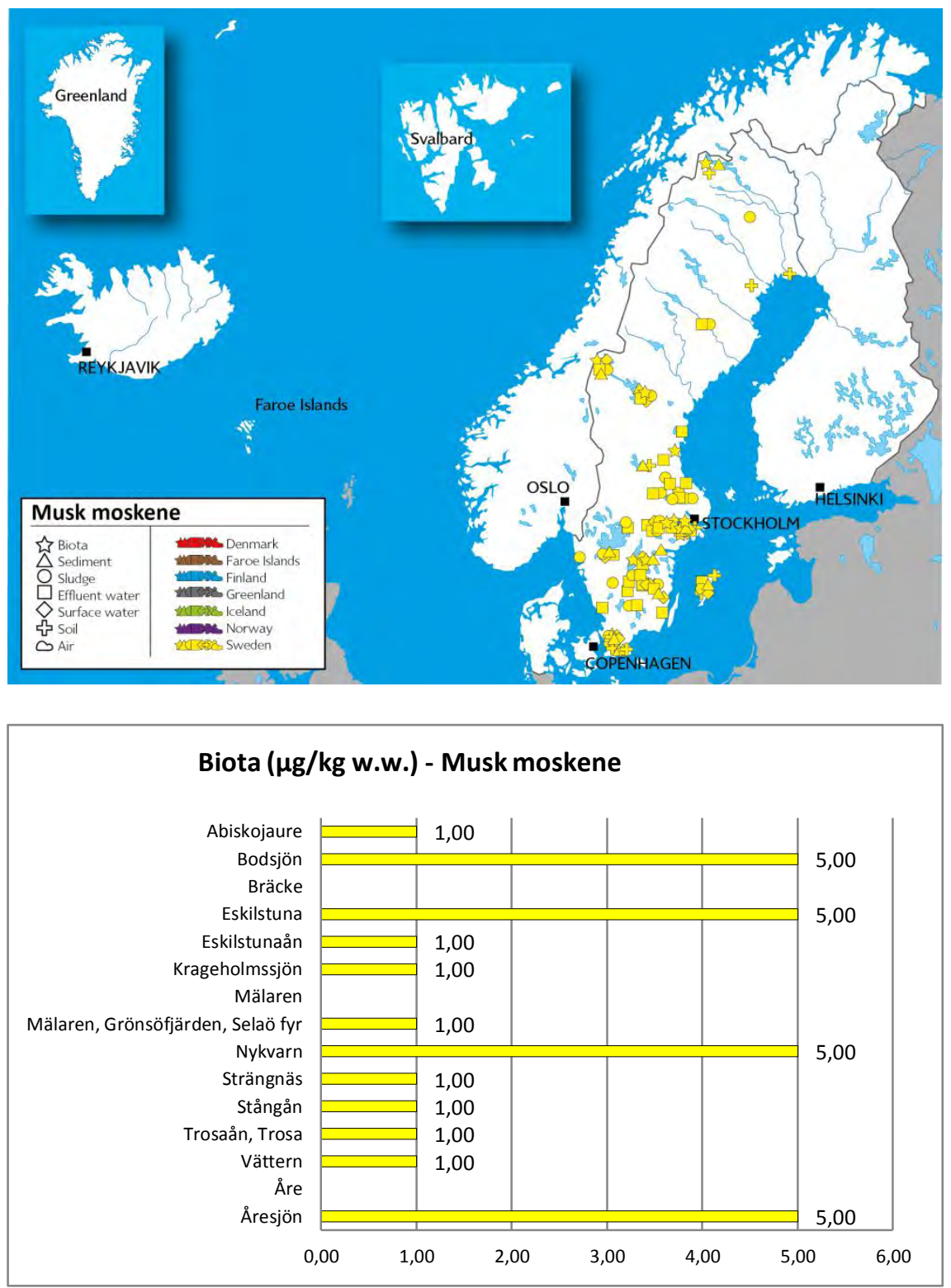


\begin{tabular}{|c|c|c|}
\hline \multirow{2}{*}{$\begin{array}{l}\text { Musk moskene } \\
\text { Matrix }\end{array}$} & \multicolumn{2}{|c|}{ Sweden } \\
\hline & $\min$ & Nr.S \\
\hline & $\max$ & (ref) \\
\hline & $A D$ & \\
\hline \multirow[t]{3}{*}{ Biota $(\mu \mathrm{g} / \mathrm{kg} w w)$} & nd & $23(a b)$ \\
\hline & 5,00 & \\
\hline & 2,60 & \\
\hline \multirow[t]{3}{*}{ Sediment $(\mu \mathrm{g} / \mathrm{kg} \mathrm{dw})$} & nd & $42(a b)$ \\
\hline & 5,00 & \\
\hline & 1,31 & \\
\hline \multirow[t]{3}{*}{ STP sludge $(\mu \mathrm{g} / \mathrm{kg} \mathrm{dw})$} & nd & $36(a b)$ \\
\hline & 5,00 & \\
\hline & 1,15 & \\
\hline \multirow[t]{3}{*}{ Soil ( $\mu \mathrm{g} / \mathrm{kg} \mathrm{dw)}$} & nd & $35(a b)$ \\
\hline & 16 & \\
\hline & 3,79 & \\
\hline \multirow[t]{3}{*}{ STP effluent (ng/l) } & nd & $51(a b)$ \\
\hline & 10 & \\
\hline & 1,25 & \\
\hline \multirow[t]{3}{*}{ Ground water (ng/l) } & 1,00 & $3(a b)$ \\
\hline & 1,00 & \\
\hline & 1,00 & \\
\hline \multirow[t]{3}{*}{ STP Influent (ng/l) } & nd & $27(a b)$ \\
\hline & 10 & \\
\hline & 3,88 & \\
\hline \multirow[t]{3}{*}{ Leachate (ng/l) } & nd & $4(a b)$ \\
\hline & 5,00 & \\
\hline & 2,33 & \\
\hline \multirow[t]{3}{*}{ Storm water (ng/l) } & 1,00 & $4(a)$ \\
\hline & 1,00 & \\
\hline & 1,00 & \\
\hline \multirow[t]{3}{*}{ Surface water (ng/l) } & nd & $34(a b)$ \\
\hline & 3,00 & \\
\hline & 1,11 & \\
\hline
\end{tabular}

References:

a) (Törneman, 2008)

b) (Törneman, 2010)

\section{Conclusion:}

The compound was only monitored in Sweden and was detected in all locations monitored. 


\subsubsection{Musk tibetene}

CAS nr.: 145-39-1

Info: Synthetic fragrance in perfumes and cosmetics

Toxicity data: NA

$P N E C=\mathrm{NA}$
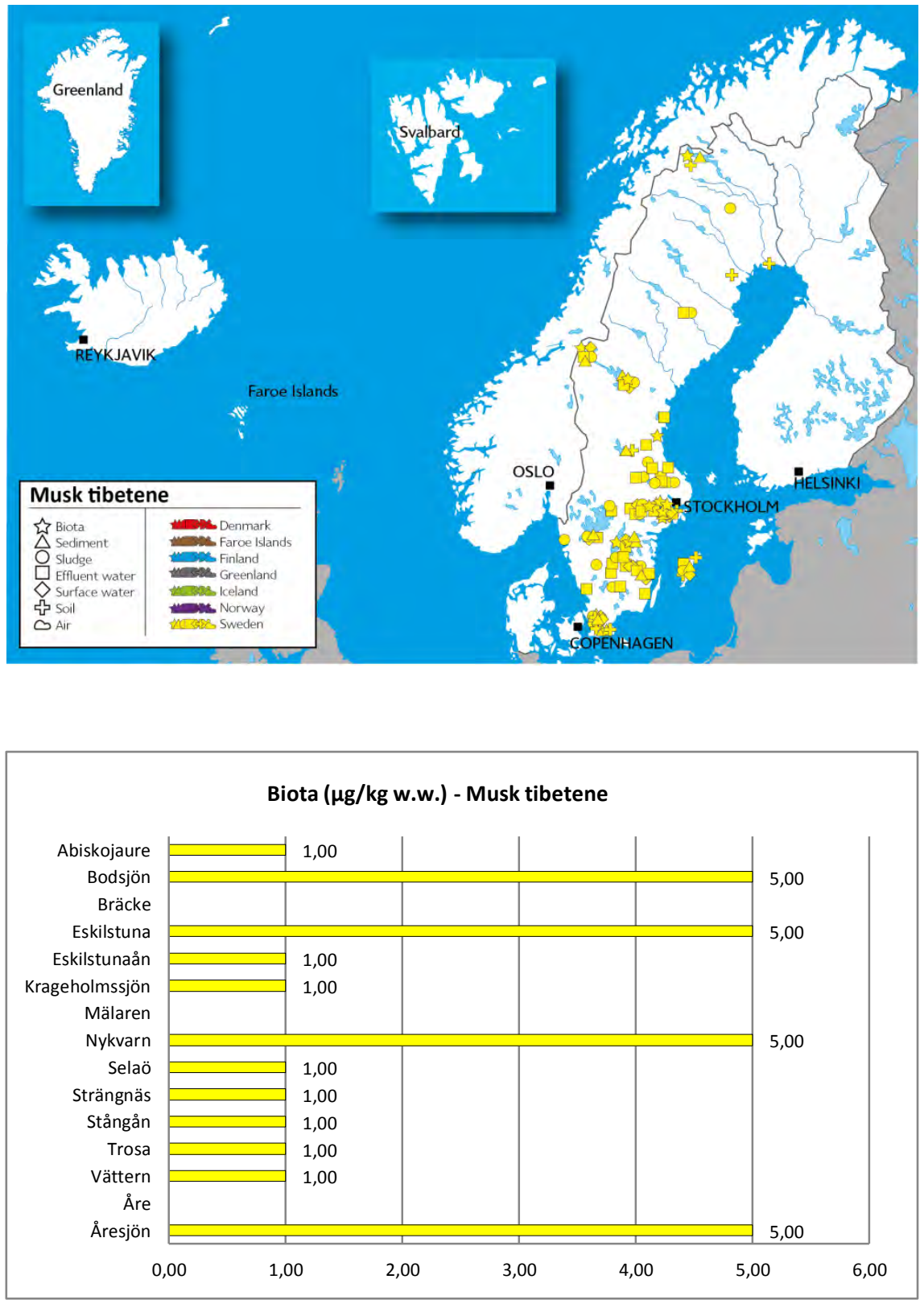


\begin{tabular}{|c|c|c|}
\hline \multirow{2}{*}{$\begin{array}{l}\text { Musk tibetene } \\
\text { Matrix }\end{array}$} & \multicolumn{2}{|c|}{ Sweden } \\
\hline & $\min$ & Nr.S \\
\hline & $\max$ & (ref) \\
\hline & $A D$ & \\
\hline \multirow[t]{3}{*}{ Biota ( $\mu \mathrm{g} / \mathrm{kg} w w)$} & nd & $23(a b)$ \\
\hline & 5,00 & \\
\hline & 2,60 & \\
\hline \multirow[t]{3}{*}{ Sediment $(\mu \mathrm{g} / \mathrm{kg} \mathrm{dw})$} & nd & $42(a b)$ \\
\hline & 5,00 & \\
\hline & 1,29 & \\
\hline \multirow[t]{3}{*}{ STP sludge $(\mu \mathrm{g} / \mathrm{kg} \mathrm{dw})$} & nd & $36(a b)$ \\
\hline & 5,00 & \\
\hline & 1,15 & \\
\hline \multirow[t]{3}{*}{ Soil $(\mu \mathrm{g} / \mathrm{kg} \mathrm{dw})$} & nd & $35(a b)$ \\
\hline & 5 & \\
\hline & 1,19 & \\
\hline \multirow[t]{3}{*}{ STP effluent (ng/l) } & nd & $51(a b)$ \\
\hline & 640 & \\
\hline & 17,25 & \\
\hline \multirow[t]{3}{*}{ Ground water (ng/l) } & 1,00 & $3(a)$ \\
\hline & 1,00 & \\
\hline & 1,00 & \\
\hline \multirow[t]{3}{*}{ STP Influent (ng/l) } & nd & $27(a b)$ \\
\hline & 1100 & \\
\hline & 125,61 & \\
\hline \multirow[t]{3}{*}{ Leachate (ng/l) } & nd & $4(a b)$ \\
\hline & 5,00 & \\
\hline & 2,33 & \\
\hline \multirow[t]{3}{*}{ Storm water (ng/l) } & 1,00 & 4 (a) \\
\hline & 1,00 & \\
\hline & 1,00 & \\
\hline \multirow[t]{3}{*}{ Surface water (ng/l) } & nd & $34(a b)$ \\
\hline & 640 & \\
\hline & 75,68 & \\
\hline
\end{tabular}

References:

a) (Törneman, 2008)

b) (Törneman, 2010)

\section{Conclusion:}

The compound was only monitored in Sweden and was detected in all locations monitored. 


\subsubsection{Phantolide}

CAS nr.: 15323-35-0

Info: Phantolide is a polycyclic musk, a group of synthetic fragrance chemicals typically used in cosmetics, perfumes, air fresheners, cleaning products, detergents, soap, and many other everyday products with artificial scents

Toxicity data: NOEC and LOEC $\min 0,044 \mathrm{mg} / \mathrm{l}$, $\max 0,90 \mathrm{mg} / \mathrm{l}$

(Törneman, 2010)

$P N E C=\mathrm{NA}$
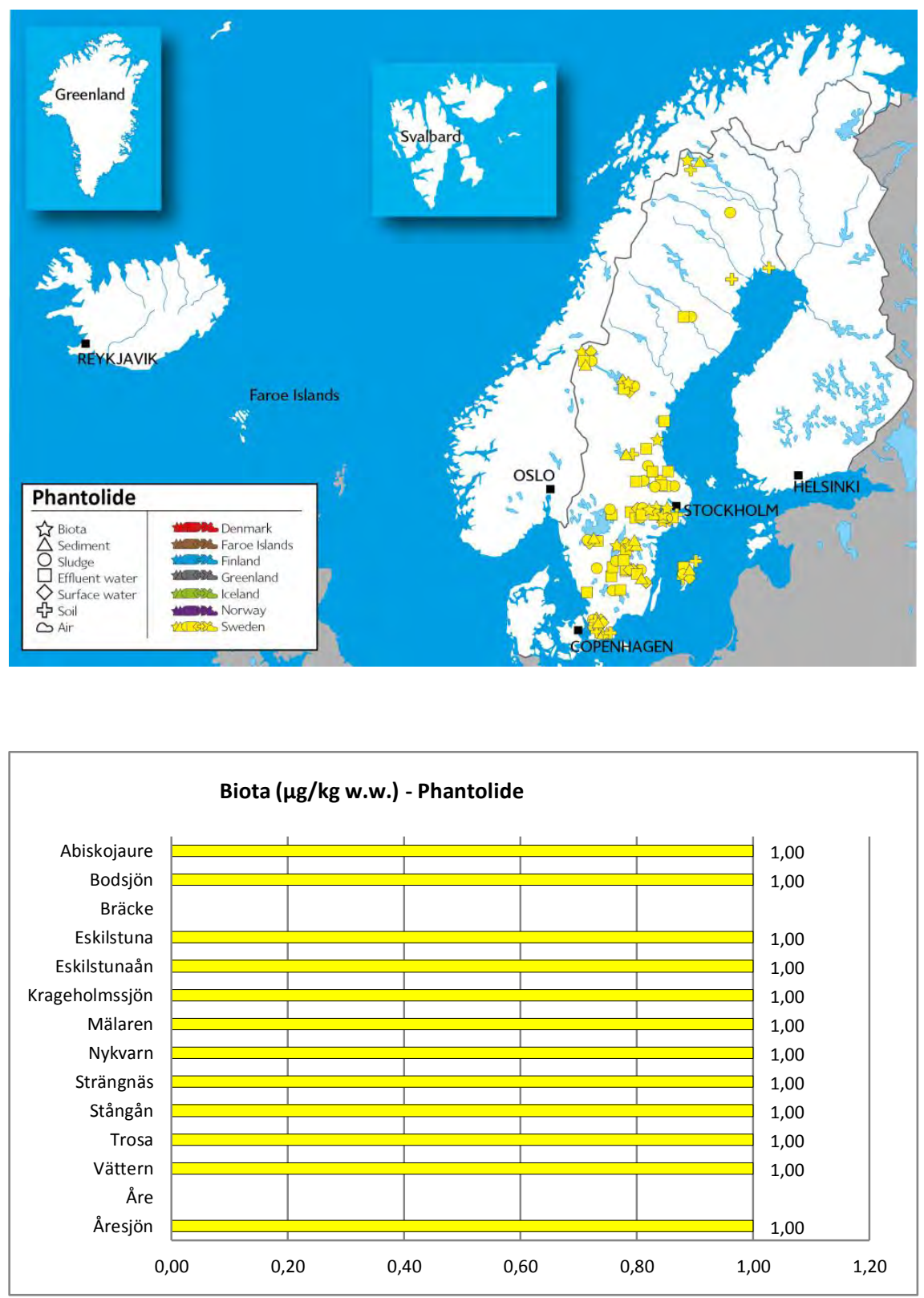


\begin{tabular}{|c|c|c|}
\hline & Sw & \\
\hline \multirow[t]{3}{*}{ Matrix } & $\min$ & Nr.S \\
\hline & $\max$ & (ref) \\
\hline & $A D$ & \\
\hline \multirow[t]{3}{*}{ Biota ( $\mu \mathrm{g} / \mathrm{kg} w w)$} & nd & $23(a b)$ \\
\hline & 1,00 & \\
\hline & 1,00 & \\
\hline \multirow[t]{3}{*}{ Sediment $(\mu \mathrm{g} / \mathrm{kg} \mathrm{dw})$} & nd & $42(a b)$ \\
\hline & 5,00 & \\
\hline & 1,29 & \\
\hline \multirow[t]{3}{*}{ STP sludge $(\mu \mathrm{g} / \mathrm{kg} \mathrm{dw})$} & nd & $36(a b)$ \\
\hline & 29,9 & \\
\hline & 4,81 & \\
\hline \multirow[t]{3}{*}{ Soil ( $\mu \mathrm{g} / \mathrm{kg} \mathrm{dw})$} & nd & $35(a b)$ \\
\hline & 5,00 & \\
\hline & 1,19 & \\
\hline \multirow[t]{3}{*}{ STP effluent (ng/l) } & nd & $51(a b)$ \\
\hline & 2,00 & \\
\hline & 1,04 & \\
\hline \multirow[t]{3}{*}{ Ground water (ng/l) } & 1,00 & 3 (a) \\
\hline & 1,00 & \\
\hline & 1,00 & \\
\hline \multirow[t]{3}{*}{ STP Influent (ng/l) } & nd & $27(a b)$ \\
\hline & 10 & \\
\hline & 3,36 & \\
\hline \multirow[t]{3}{*}{ Leachate (ng/l) } & nd & $4(a b)$ \\
\hline & 5,00 & \\
\hline & 2,33 & \\
\hline \multirow[t]{3}{*}{ Storm water (ng/l) } & 1,00 & $4(a)$ \\
\hline & 1,00 & \\
\hline & 1,00 & \\
\hline \multirow[t]{3}{*}{ Surface water (ng/l) } & nd & $34(a b)$ \\
\hline & 3,00 & \\
\hline & 1,10 & \\
\hline
\end{tabular}

References:

a) (Törneman, 2008)

b) (Törneman, 2010)

\section{Conclusion:}

The compound was only monitored in Sweden and was detected in all locations monitored. 


\subsubsection{Tonalide}

CAS nr.: 21145-77-7

Info: Synthetic fragrance in cosmetics, detergents, toiletries, cigarettes Toxicity data: NOEC and LOEC max 0,14 mg/l (Törneman, 2010)

$P N E C=$

PNEC aqua $0,35 \mu \mathrm{g} / \mathrm{l}$

PNEC sediment $200 \mu \mathrm{g} / \mathrm{l}$

(Törneman, 2010)
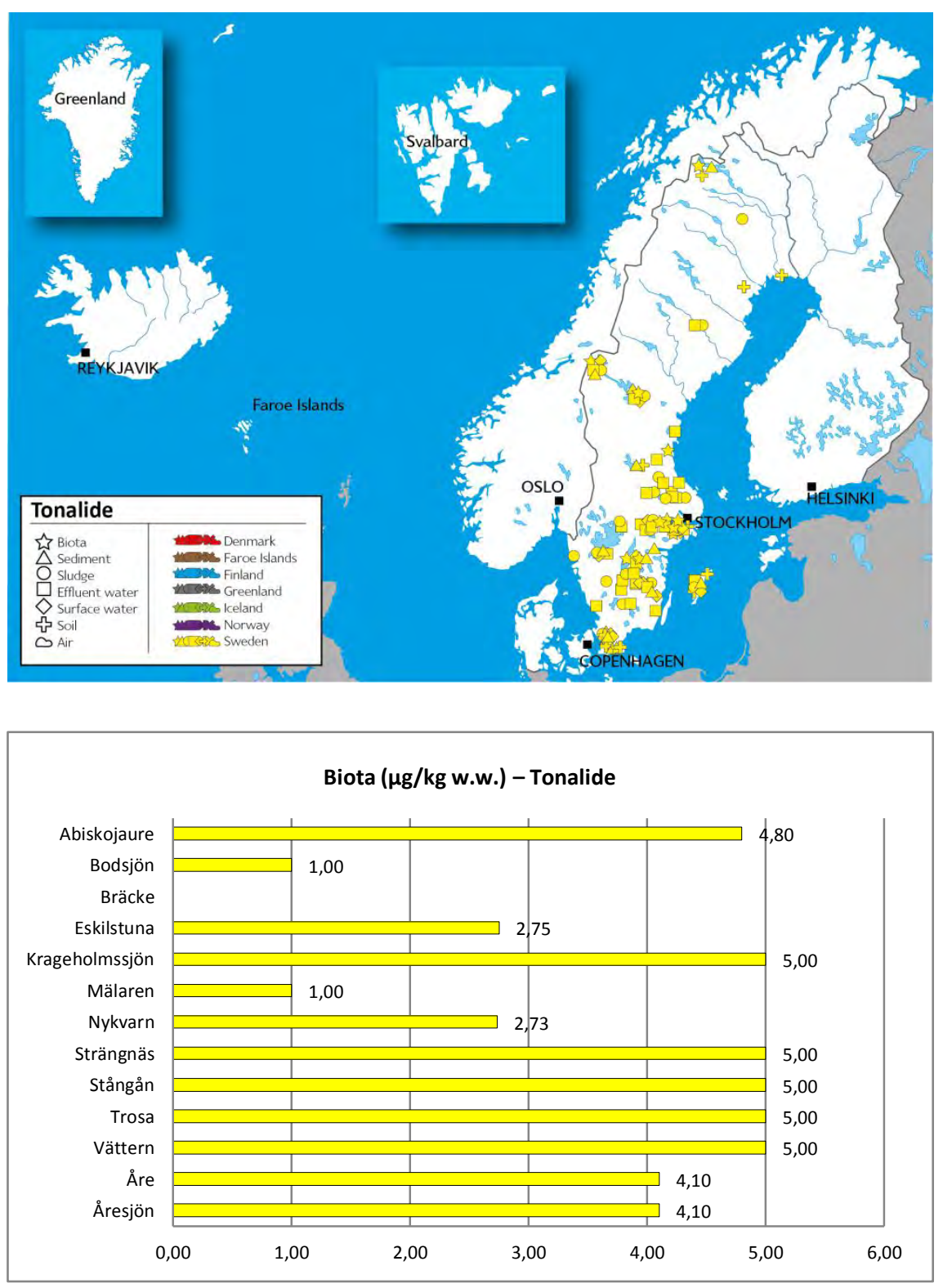


\begin{tabular}{|c|c|c|}
\hline Tonalide & \multicolumn{2}{|c|}{ Sweden } \\
\hline \multirow[t]{3}{*}{ Matrix } & $\min$ & Nr.S \\
\hline & $\max$ & (ref) \\
\hline & $A D$ & \\
\hline \multirow[t]{3}{*}{ Biota $(\mu \mathrm{g} / \mathrm{kg} w w)$} & nd & \multirow{3}{*}{$23(a b)$} \\
\hline & 5,00 & \\
\hline & 3,49 & \\
\hline Sediment ( & nd & \multirow{3}{*}{$42(a b)$} \\
\hline \multirow[t]{2}{*}{$\mu \mathrm{g} / \mathrm{kg} \mathrm{dw})$} & 28 & \\
\hline & 5,18 & \\
\hline \multirow[t]{3}{*}{ STP sludge $(\mu \mathrm{g} / \mathrm{kg} \mathrm{dw})$} & 1,00 & \multirow[b]{3}{*}{$36(a b)$} \\
\hline & 2560 & \\
\hline & 538,15 & \\
\hline \multirow[t]{3}{*}{ Soil ( $\mu \mathrm{g} / \mathrm{kg} \mathrm{dw})$} & nd & \multirow{3}{*}{$35(a b)$} \\
\hline & 97 & \\
\hline & 11,41 & \\
\hline \multirow[t]{3}{*}{ STP effluent (ng/l) } & 1,00 & \multirow{3}{*}{$51(a b)$} \\
\hline & 1570 & \\
\hline & 67,28 & \\
\hline \multirow[t]{3}{*}{ Ground water (ng/l) } & 1,00 & \multirow{3}{*}{$3(a)$} \\
\hline & 1,00 & \\
\hline & 1,00 & \\
\hline \multirow[t]{3}{*}{ STP Influent (ng/l) } & nd & \multirow{3}{*}{$27(a b)$} \\
\hline & 380 & \\
\hline & 146,46 & \\
\hline \multirow[t]{3}{*}{ Leachate (ng/l) } & nd & \multirow{3}{*}{$4(a b)$} \\
\hline & 5,00 & \\
\hline & 2,33 & \\
\hline \multirow[t]{3}{*}{ Storm water (ng/l) } & 1,00 & \multirow{3}{*}{4 (a) } \\
\hline & 1,00 & \\
\hline & 1,00 & \\
\hline \multirow[t]{3}{*}{ Surface water(ng/l) } & nd & \multirow{3}{*}{$34(a b)$} \\
\hline & 32 & \\
\hline & 5,94 & \\
\hline
\end{tabular}

References:

a) (Törneman, 2008)

b) (Törneman, 2010)

\section{Conclusion:}

The compound was only monitored in Sweden and was detected in all locations monitored. The values detected in STP effluent and surface water were above the PNEC. The value detected in sediment was below the PNEC. 


\subsubsection{Traseolide}

CAS nr.: 68140-48-7

Info: Synthetic fragrance in cosmetics, detergents, toiletries, and toys Toxicity data: NA

$P N E C=\mathrm{NA}$
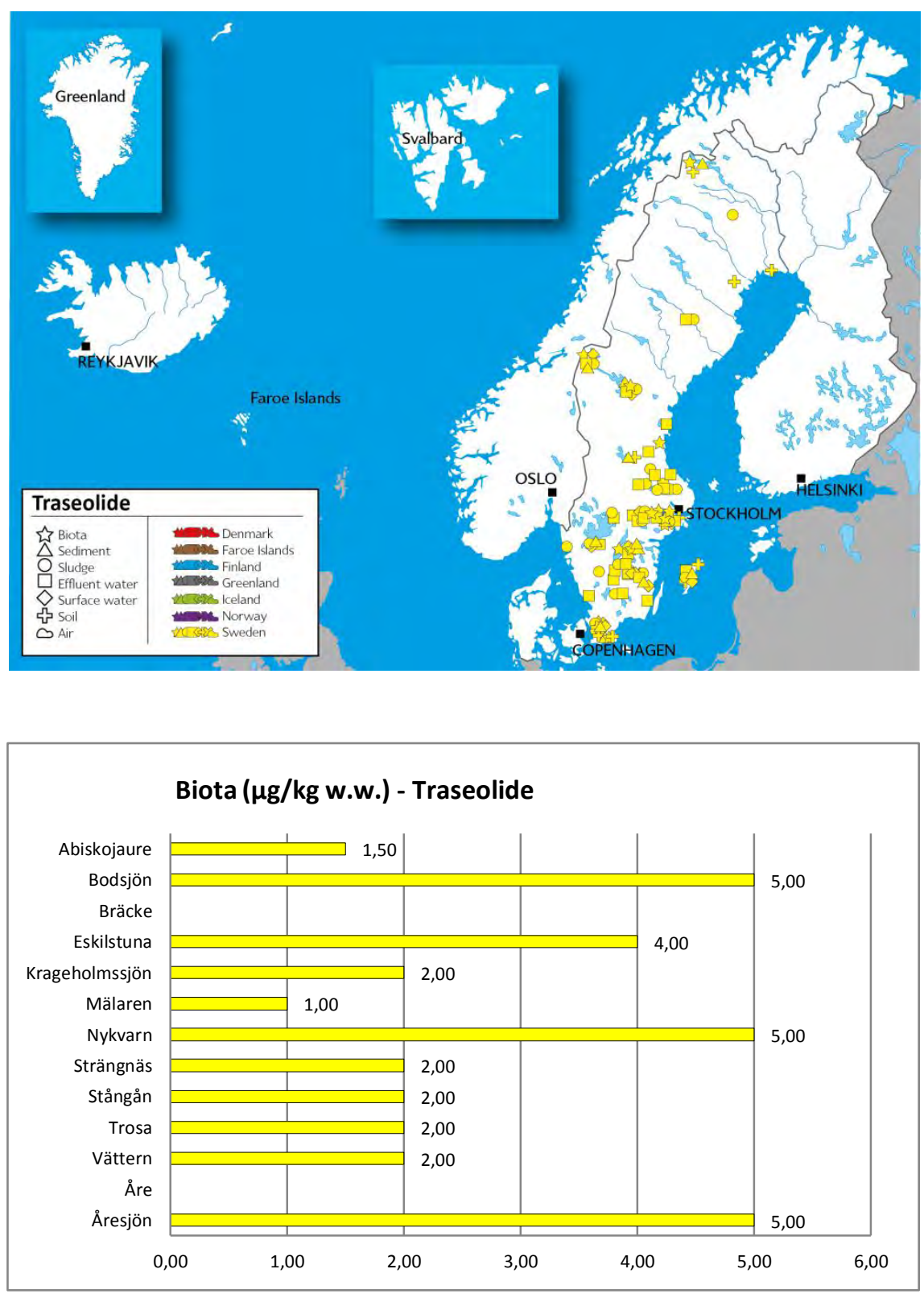


\begin{tabular}{|c|c|c|}
\hline Traseolide & \multicolumn{2}{|c|}{ Sweden } \\
\hline \multirow[t]{3}{*}{ Matrix } & $\min$ & Nr.S \\
\hline & $\max$ & (ref) \\
\hline & $A D$ & \\
\hline \multirow[t]{3}{*}{ Biota ( $\mu \mathrm{g} / \mathrm{kg} w w)$} & nd & \multirow{3}{*}{$23(a b)$} \\
\hline & 5,00 & \\
\hline & 3,07 & \\
\hline \multirow[t]{3}{*}{ Sediment $(\mu \mathrm{g} / \mathrm{kg} \mathrm{dw})$} & nd & \multirow{3}{*}{$42(a b)$} \\
\hline & 5,00 & \\
\hline & 1,29 & \\
\hline \multirow[t]{3}{*}{ STP sludge $(\mu \mathrm{g} / \mathrm{kg} \mathrm{dw})$} & nd & \multirow[b]{3}{*}{$36(a b)$} \\
\hline & 112 & \\
\hline & 23,73 & \\
\hline \multirow[t]{3}{*}{ Soil ( $\mu \mathrm{g} / \mathrm{kg} \mathrm{dw})$} & nd & \multirow{3}{*}{$35(a b)$} \\
\hline & 5,00 & \\
\hline & 1,19 & \\
\hline \multirow[t]{3}{*}{ STP effluent (ng/l) } & nd & \multirow{3}{*}{$51(a b)$} \\
\hline & 12 & \\
\hline & 1,93 & \\
\hline \multirow[t]{3}{*}{ Groundwater (ng/l) } & 1,00 & \multirow{3}{*}{3 (a) } \\
\hline & 1,00 & \\
\hline & 1,00 & \\
\hline \multirow[t]{3}{*}{ STP Influent (ng/l) } & nd & \multirow{3}{*}{$27(a b)$} \\
\hline & 10 & \\
\hline & 4,18 & \\
\hline \multirow[t]{3}{*}{ Leachate (ng/l) } & nd & \multirow{3}{*}{$4(a b)$} \\
\hline & 5,00 & \\
\hline & 2,33 & \\
\hline \multirow[t]{3}{*}{ Storm water (ng/l) } & 1,00 & \multirow{3}{*}{4 (a) } \\
\hline & 1,00 & \\
\hline & 1,00 & \\
\hline \multirow[t]{3}{*}{ Surface water (ng/l) } & nd & \multirow{3}{*}{$34(a b)$} \\
\hline & 5,5 & \\
\hline & 1,32 & \\
\hline
\end{tabular}

References:

a) (Törneman, 2008)

b) (Törneman, 2010)

\section{Conclusion:}

The compound was only monitored in Sweden and was detected in all locations monitored. 


\subsection{Compounds monitored in one study}

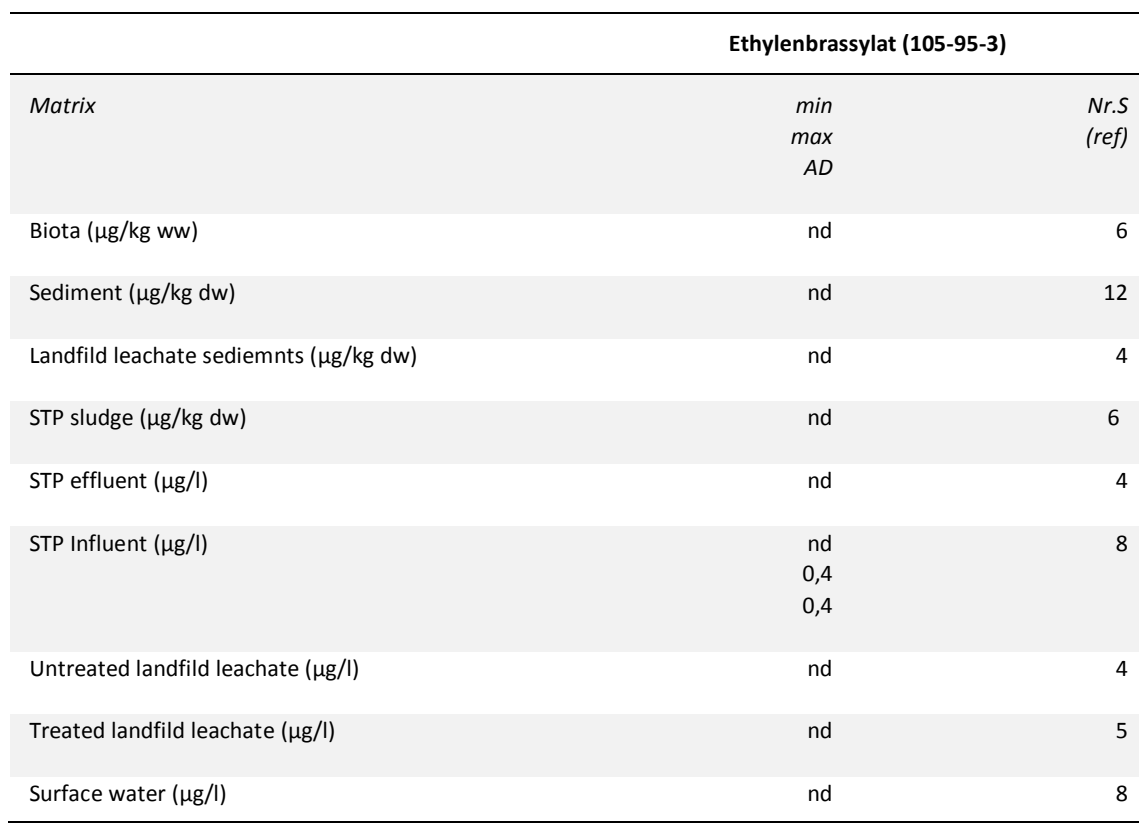

\section{Reference:}

- (Møskeland, et al., 2006)

\begin{tabular}{|c|c|c|c|c|}
\hline \multirow[b]{2}{*}{ Matrix } & \multicolumn{2}{|c|}{ D-limonene (5989-27-5) } & \multicolumn{2}{|c|}{ L-limonene (95327-98-3) } \\
\hline & $\min$ & Nr.S & $\min$ & Nr.S \\
\hline & $\max$ & (ref) & $\max$ & (ref) \\
\hline & $A D$ & & $A D$ & \\
\hline \multirow[t]{2}{*}{ Biota $(\mu \mathrm{g} / \mathrm{kg} w w)$} & nd & 2 & & \\
\hline & $\begin{array}{l}\text { nd } \\
\text { nd }\end{array}$ & & nd & 2 \\
\hline \multirow[t]{3}{*}{ Sediment (ng/kg dw) } & nd & 6 & nd & \\
\hline & 100 & & 260 & 6 \\
\hline & 100 & & 98 & \\
\hline \multirow[t]{3}{*}{ STP sludge (ng/kg dw) } & nd & & nd & \multirow{3}{*}{21} \\
\hline & 2600 & & 16000 & \\
\hline & 699,3 & & 1840,3 & \\
\hline STP effluent & nd & 2 & nd & 2 \\
\hline Leachate & nd & 1 & nd & 1 \\
\hline \multirow[t]{3}{*}{ Surface water $(\mu \mathrm{g} / \mathrm{l})$} & nd & 2 & nd & \multirow{3}{*}{2} \\
\hline & 0,18 & & 0,51 & \\
\hline & 0,18 & & 0,51 & \\
\hline \multirow[t]{3}{*}{ Air $(\mu \mathrm{g} / \mathrm{m} 3) \mathrm{d}$-limonene and I-limonene } & 2,10 & & & \multirow{3}{*}{3} \\
\hline & 2,80 & & & \\
\hline & 2,33 & & & \\
\hline
\end{tabular}

\section{Reference:}

- (Potter, et al., 2004) 


\section{UV-filter compounds}

\subsection{Scope and definition}

UV-filters are defined as compounds used to prevent UV light from getting through various materials. Within the PPCPs, UV-filters are used as sunscreen to protect the skin, by reducing the amount of UV light that reaches the skin, in order to prevent the negative health impacts of these rays.

UV-filters used in PPCPs are believed to emit to the environment primarily from losses due to washing and bathing.

\begin{tabular}{ll} 
Table 6: UV-filter compounds monitored in the Nordic countries \\
\hline Compound & CAS \\
\hline 3-Benzylidene camphor (3-BC) & $15087-24-8$ \\
2-Ethylhexyl 4-dimethylaminobenzoate (EPABA ,ODPABA) & $21245-02-3$ \\
Diethylaminohydroxybenzoyl hexyl benzoate (DBENZO,) & $302776-68-7$ \\
Ethylhexylmethoxycinnamate & $5466-77-3$ \\
Butylmethoxydibenzoylmethane (Avobenzone - Eusolex 9020 - BMDB) & $70356-09-1$ \\
Isoamyl p-methoxycinnamate (IMC-IAMC) & $71617-10-2$ \\
Homosalate (HMS - 3,3,5-trimehtylcyclohexylsalicylate) & $118-56-9$ \\
2-Ethylhexyl salicylate (EHS) & $118-60-5$ \\
4-Methyl benzylidene camphor (Eusolex 6300) & $36861-47-9 / 38102-62-4$ \\
Octocrylene (2-ethylhexyl 2-cyano-3,3-di(phenyl)prop-2-enoate) & $6197-30-4$ \\
2,4-Dihydroxybensophenone & $131-56-6$ \\
2,2'-Dihydroxy-4-metoxybensophenone (DHMB) & $131-53-3$ \\
Benzophenone-3, Eusolex 4360 & $131-57-7$
\end{tabular}




\subsection{Compounds monitored in at least two studies}

\subsubsection{Butylmethoxydibenzoylmethane, Avobenzone Eusolex 9020 (BMDB)}

CAS nr.: 70356-09-1

Info: Oil soluble ingredient used in sunscreen products to absorb the full spectrum of UVA rays

Toxicity data: NA

$P N E C=$ NA

\begin{tabular}{|c|c|c|c|c|}
\hline \multirow{2}{*}{$\begin{array}{l}\text { BMDB } \\
\text { Matrix }\end{array}$} & \multicolumn{2}{|c|}{ Norway } & \multicolumn{2}{|c|}{ Sweden } \\
\hline & $\min$ & Nr.S & $\min$ & Nr.S \\
\hline & $\max$ & (ref) & $\max$ & (ref) \\
\hline & $A D$ & & $A D$ & \\
\hline Biota (ng/g ww) & nd & $10(a b)$ & nd & 5 (c) \\
\hline Sediment (ng/g dw) & nd & $17(a b)$ & nd & 7 (c) \\
\hline Landfild leachate sediments ( $\mathrm{ng} / \mathrm{g} \mathrm{dw}$ ) & nd & 4 (a) & & \\
\hline STP sludge (ng/g dw) & nd & $10(a b)$ & nd & 8 (c) \\
\hline \multirow[t]{3}{*}{ STP effluent (ng/l) } & nd & 4 (b) & nd & 8 (c) \\
\hline & & & 14 & \\
\hline & & & 4,67 & \\
\hline STP influent & nd & 8 (b) & & \\
\hline Untreated landfill leachates (ng/l) & nd & 4 (b) & & \\
\hline Treated landfill leachates (ng/l) & nd & 5 (b) & & \\
\hline Urban run-off & & & nd & 6 (c) \\
\hline \multirow[t]{3}{*}{ Surface water (ng/l) } & nd & 8 (b) & nd & 18 (c) \\
\hline & & & 9,90 & \\
\hline & & & 6,14 & \\
\hline
\end{tabular}

\section{References:}

a) (Schlabach, et al., 2009)

b) (Møskeland, et al., 2006)

c) (Remberger, et al., 2011)

\section{Conclusion:}

The compound was monitored in Norway and Sweden. It was not detected in any matrix in Norway, but was detected in STP effluent water and surface water in Sweden.

\subsection{Compounds monitored in one study}




\begin{tabular}{|c|c|c|c|c|c|c|c|c|c|c|c|c|c|}
\hline & $\begin{array}{l}\text { Octocrylene } \\
(6197-30-4)\end{array}$ & $\begin{array}{r}\text { 3-Benzyl- } \\
\text { idene } \\
\text { camphor } \\
(15087-24-8)\end{array}$ & $\begin{array}{r}\text { 4-Methyl } \\
\text { benzylidene } \\
\text { camphor, } \\
(36861-47-9)\end{array}$ & $\begin{array}{r}\text { Benzo- } \\
\text { phenone-3, } \\
\text { Eusolex } 4360 \\
(131-57-7)\end{array}$ & $\begin{array}{l}\text { Ethylhexyl- } \\
\text { methoxy- } \\
\text { cinnamate } \\
(5466-77-3)\end{array}$ & $\begin{array}{r}\text { Diethylamino- } \\
\text { hydroxy- } \\
\text { benzoylhexyl } \\
\text { benzoate } \\
(302776-68-7)\end{array}$ & $\begin{array}{r}\text { Ethylhexyl } \\
\text { salicylate } \\
(118-60-5)\end{array}$ & $\begin{array}{r}\text { 2-Ethylhexyl } \\
\text { 4-(dimethyl- } \\
\text { amino) } \\
\text { benzoate } \\
(21245-02-3)\end{array}$ & $\begin{array}{r}\text { Isoamyl } \\
\text { pmethoxy- } \\
\text { cinnamate } \\
(71617-10-2)\end{array}$ & $\begin{array}{r}\text { 2,4-Di- } \\
\text { hydroxy- } \\
\text { benso- } \\
\text { phenone } \\
(131-56-6)\end{array}$ & $\begin{array}{r}\text { Homosalate } \\
(118-56-9)\end{array}$ & $\begin{array}{r}2,2^{\prime} \text {-Dihydroxy- } \\
\text { 4- metoxyben- } \\
\text { sophenone } \\
(131-53-3)\end{array}$ & \\
\hline \multirow[t]{3}{*}{ Matrix } & $\min$ & $\min$ & $\min$ & $\min$ & $\min$ & $\min$ & $\min$ & $\min$ & $\min$ & $\min$ & $\min$ & $\min$ & Nr.S \\
\hline & $\max$ & $\max$ & $\max$ & $\max$ & $\max$ & $\max$ & $\max$ & $\max$ & $\max$ & $\max$ & $\max$ & $\max$ & \\
\hline & $A D$ & $A D$ & $A D$ & $A D$ & $A D$ & $A D$ & $A D$ & $A D$ & $A D$ & $A D$ & $A D$ & $A D$ & \\
\hline \multirow[t]{3}{*}{ Biota (ng/g ww) } & $\mathrm{nd}$ & nd & nd & nd & nd & nd & nd & nd & nd & nd & nd & nd & 5 \\
\hline & 6,80 & & 1,20 & & 0,80 & & & & 0,12 & & & & \\
\hline & 4,33 & & 1,20 & & 0,60 & & & & 0,12 & & & & \\
\hline \multirow[t]{3}{*}{ Sediment (ng/g dw) } & nd & nd & nd & nd & nd & nd & nd & nd & nd & nd & nd & nd & 7 \\
\hline & 350 & 62 & 29 & 11 & 45 & & 23 & 1,20 & 1,80 & 4,3 & 32 & & \\
\hline & 125,25 & 35,50 & 17,90 & 8,75 & 27,33 & & 23 & 1,20 & 1,21 & 2,72 & 9,76 & & \\
\hline \multirow[t]{3}{*}{ STP sludge (ng/g dw) } & 2700 & nd & 320 & 14 & 31 & $n d$ & nd & nd & nd & nd & 140 & nd & 8 \\
\hline & 9100 & 64 & 1300 & 82 & 410 & 2500 & & 39 & 7,9 & 89 & 950 & 2,8 & \\
\hline & 6287,5 & 64 & 762,5 & 39,38 & 160,4 & 1635,7 & & 13,86 & 3,98 & 28 & 577,5 & 1,2 & \\
\hline \multirow[t]{3}{*}{ STP effluent (ng/l) } & nd & nd & nd & 14 & nd & nd & nd & nd & nd & nd & nd & nd & 8 \\
\hline & 600 & 19 & 230 & 360 & 49 & 270 & 12 & 160 & 2 & 68 & 25 & 3,3 & \\
\hline & 189,80 & 19 & 62 & 131,2 & 21,50 & 96,70 & 12 & 81,35 & 2 & 37,32 & 6,97 & 2,35 & \\
\hline \multirow[t]{3}{*}{ Urban run-off (ng/l) } & nd & nd & nd & 2,50 & nd & nd & nd & nd & nd & nd & nd & nd & 6 \\
\hline & 14 & & 430 & 13 & 1,6 & 11 & & 38 & & 3,50 & 25 & 0,54 & \\
\hline & 8,84 & & 129,1 & 4,77 & 1,60 & 8 & & 13,46 & & 3,40 & 6,97 & 0,52 & \\
\hline \multirow[t]{3}{*}{ Surface water (ng/l) } & nd & nd & nd & 0,60 & nd & nd & nd & nd & nd & nd & nd & nd & 18 \\
\hline & 1200 & & 35 & 34 & 15 & 960 & 89 & 46 & 0,84 & 11 & 46 & 2,3 & \\
\hline & 156,64 & & 14,49 & 7,21 & 6,44 & 136,5 & 32,15 & 9,81 & 0,82 & 4,27 & 7,84 & 0,8 & \\
\hline
\end{tabular}

\section{Reference:}

a) (Remberger, et al., 2011) 



\section{Mosquito repellents}

This category is defined as pesticides for human consumption (skin application), used specifically to repel mosquitos. No other pesticides are included.

$\mathrm{N}, \mathrm{N}$-dietyl-m-toluamid (DEET) is the only mosquito repellant monitored in the Nordic countries.

DEET has been found to inhibit the activity of a central nervous system enzyme, acetylcholinesterase, in both insects and mammals.

\subsubsection{N,N-diethyl-m-toluamid (DEET)}

CAS nr.: 134-62-3

Info: It is the most common active ingredient in insect repellents Toxicity data: NA

PNEC aqua $=43 \mu \mathrm{g} / \mathrm{l}$ (Törneman , 2011b)

Reference: (Törneman , 2011b)

\begin{tabular}{|c|c|c|}
\hline DEET & & \\
\hline \multirow[t]{3}{*}{ Matrix } & Min & Nr.S \\
\hline & $\max$ & (ref) \\
\hline & $A D$ & \\
\hline \multirow[t]{3}{*}{ Sediment (mg/kg dw) } & nd & 4 \\
\hline & 3,80 & \\
\hline & 3,80 & \\
\hline STP sludge (mg/kg dw) & nd & 4 \\
\hline \multirow[t]{3}{*}{ STP effluent $(\mu \mathrm{g} / \mathrm{l})$} & 0,01 & 14 \\
\hline & 0,70 & \\
\hline & 0,17 & \\
\hline \multirow[t]{3}{*}{ STP influent $(\mu \mathrm{g} / \mathrm{l})$} & nd & 6 \\
\hline & 0,58 & \\
\hline & 0,33 & \\
\hline \multirow[t]{3}{*}{ Groundwater $(\mu \mathrm{g} / \mathrm{l})$} & nd & 8 \\
\hline & 0,06 & \\
\hline & 0,03 & \\
\hline \multirow[t]{3}{*}{ Surface water $(\mu \mathrm{g} / \mathrm{l})$} & nd & 11 \\
\hline & 1,10 & \\
\hline & 0,25 & \\
\hline
\end{tabular}




\section{Conclusion:}

The compound has only been monitored in one study in Sweden. It has been detected in sediment, groundwater, surface water, STP effluent and STP influent. All water samples are below the PNEC value. 


\section{Observations}

This report has given an overview of monitoring data for certain PPCPs in the Nordic countries. The scope of the report has not been to draw any conclusions, but the authors would like to present some observations made during the research phase:

- The number of reports produced on this subject was small, compared to several other niches of environmental monitoring. However, the amount of data was very high

- The majority of all data is provided by studies carried out in Sweden. Data for Finland, the Faroe Islands, Greenland and Iceland are scarcer

- Most of the monitoring for these compounds is carried out in STPs and STP recipients. Results from biota and abiotic matrices from more distant location to large human settlements are scarce

Even if this report provides a unique overview of PPCPs in the Nordic environment - it also provides insight into obvious knowledge gaps. This project could therefore be extended in several ways:

- A second step to this report could be to prioritize these compounds by identifying those that have been detected in the environment in concentrations above PNEC values. This could be interesting to assess which compounds could require further monitoring and possibly more regulation relative to their use

- The monitoring reports reviewed include many other PPCPs, that belong to other categories than those considered in this report. It could be of interest to process the monitoring data as done in this report

- During the production of this report, the authors have observed numerous studies from outside of the Nordic countries covering PPCPs. It could be interesting to assess which compounds are monitored in other regions of the world and not in the Nordic region and correlate the latter with sales statistics for those products in the Nordic area

Having an overview such as this report on monitoring data in neighboring countries is of interest for environmental authorities and law makers. In this manner, it could be of interest for the Nordic council to create 
an online database, where each country could enter their monitoring data available for environmental pollutants of interest. Throughout time, this would allow countries to also follow trends, and observe the effect of contingency actions in each country. 


\section{Bibliography}

Adolfsson-Erici, M., Johansson, C. \& Petterson, M., 2003. Screening av triclosan $i$ reningsverk och recipienter, s.l.: Naturvårdsverket.

Andersson, J. et al., 2006. IVL report B1689: Results from the Swedish National Screening Programme 2005 Subreport 1: Antibiotics, Anti-inflammatory substances, and Hormones, s.l.: IVL.

Bakke, T. et al., 2008. TA 1021: Mapping selected organic contaminants in the Barents Sea 2007., s.l.: Klif (SFT).

Evenset, A. et al., 2009. TA-2510: Screening of new contaminants in samples from the Norwegian Arctic, s.l.: Klif.

faroe, NA. s.l.: s.n.

Fick, J., Lindberg, R., Kay, L. \& Brorström-Lundén, E., 2011. Preliminary report: Results from the Swedish National Screening Programme 2010 - Subreport 3. Pharmaceuticals, s.l.: s.n.

Fjeld, E. et al., 2006. TA 2077:Kartlegging av utvalgte nye organiske miljøgifter bromerte flammehemmere, klorerte parafiner, bisfenol A og triclosan, s.l.: SFT.

Green, N. et al., 2009. Screening of selected priority substances of the Water Framework Directive in marine samples 2004 - 2033, s.l.: s.n.

Gustavson, K. et al., 2009. Chemicals From Marine Fish Farms, s.l.: TemaNord.

Haglund, P. \& Olofsson, U., 2006. Miljöövervakning av slam, redovisning av resultat från 2004, 2005 och 2006 års provtagningar, s.l.: s.n.

Haglund, P. \& Olofsson, U., 2007. Miljöövervakning av slam, redovisning av resultat från 2007 års provtagning, s.l.: Naturvårdsverket.

Haglund, P. \& Olofsson, U., 2008. Miljöövervakning av slam, redovisning av resultat från 2008 års provtagning (inklusive en sammanfattning av åren 2004-2008), s.l.: Naturvårdsverket.

Hellström, A. \& Kreuger, J., 2005. Department of Environmental Assessment, Swedish University of Agricultural Sciences Rapport 2005:23, s.l.: Swedish University of Agricultural Sciences.

Huber, S. et al., NA. Pharmaceuticals og personal care products as environmental pollutants in Faroe Island, Iceland and Greenland. In prep., s.l.: s.n.

Ingerslev, F. et al., 2003. En præliminær kortlægning af steroidøstrogenudledningen fra danske rensningsanlæg, s.l.: s.n.

Johansson, M., Lindberg, R., Wennber, P. \& Tysklind, M., 2003. Redovisning från nationell miljöövervakning 2003: Screening av antibiotika i avloppsvatten, slam och fisk under 2002/2003, s.l.: Naturvårdsverket.

Kjølholt, J., Nielsen , P. \& Stuer-Lauridsen, F., 2003. Hormonforstyrrende stoffer og lægemidler i spildevand, s.l.: Miljøstyrelsen.

Lahti, M. \& Oikari, A., 2011. Pharmaceuticals in settleable particulate material in urban and non-urban waters. Chemosphere (2011), doi:10.1016/j.chemosphere.2011.06.183. 
Lee , S., Park, K., Hong, J. \& Choi, J., 2008. Ecotoxicological evaluation of octachlorostyrene in fourth instar larvae of Chironomus riparius (Diptera, Chironomidae).. Environ Toxicol Chem., 27(5), pp. 1118-27.

Lilja, K. et al., 2010. IVL B1897: Chemical and biological monitoring of sewage effluent water, s.l.: IVL.

Mogensen, B. et al., 2008. NOVANA-Screeningsundersøgelse af det akvatiske miljø, Faglig rapport fra DMU nr. 638, 2347: Lægemidler og triclosan i punktkilder og vandmiljøet., s.l.: DMU.

Møskeland, T. et al., 2006. TA-2156: Kartlegging av utvalgte forbindelser i legemidler og kosmetikk, s.l.: SFT (Klif).

Park, S. \& Choi, K., 2008. Hazard assessment of commonly used agricultural antibiotics. Ecotoxicology, 17(6), pp. 526-38.

Pedersen, B., Nielsen, U. \& Halling-Sørensen, B., 2007. Begrænsning af humane lægemiddelrester og antibiotikaresistens i spildevand med fokus på reduktion ved kilden, s.l.: Miljøministeriet.

Potter, A. et al., 2004. B1647: Results from the Swedish National Screening Programme 2004, Subreport 3: Limonene, s.l.: IVL.

Remberger, M. et al., 2011. B1971: Results from the Swedish National Screening Programme 2009 Subreport 3: UV-filters, s.l.: IVL.

Remberger, M., Sternbeck, J. \& Strömberg, K., 2002. IVL report B1477: Screening av triclosan och vissa bromerade fenoliska ämnen i Sverige, s.l.: IVL.

Remberger, M. et al., 2009. IVL B1810: Anti-inflammatory and analgesic drugs in WWTP influent and effluent streams and the occurrence in the aquatic environment, s.l.: IVL.

Remberger, M. et al., 2006. Results from the Swedish Screening 2005, subreport Biocide, s.l.: IVL.

Schlabach, M. et al., 2009. TA 1046: Environmental Screening of Selected Organic Compounds 2008 Human and hospital-use pharmaceuticals, aquaculture medicines and personal care products, s.l.: NILU report number: OR 13/2444.

Schlabach, M. et al., 2007. TA 2325: Human and veterinary pharmaceuticals, narcotics, and personal care products in the environment, s.l.: Klif.

SFT, 2006. TA-2216:Initial assessment of eleven pharmaceuticals using the EMEA guideline in Norway, s.l.: SFT (Klif).

Sternbeck, J., Österås, A. \& Josefsson, K., 2007 b. Triclosan, DEHP och klordan samlad utvärdering av svenska miljöövervakningsdata, s.l.: Naturvårdsverket.

Sternbeck, J. et al., 2007a. Screening of veterinary medicines in agricultural areas, s.l.: WSP Environmental.

Sternbeck, J. et al., 2007a. Screening of veterinary medicines in agricultural areas, s.l.: WSP Environmental.

Sternbeck, J. et al., 2007. Screening of veterinary medicines in agricultural areas, s.l.: WSP Environmental.

Stuer-Lauridsen, F., Halling-Sørensen, B. \& Overgaard, .., 2011. Miljøvurdering af speciallægemidler i spildevand fra sygehuse - APPENDIKSER, s.l.: Naturstyrelsen.

Stuer-Lauridsen, F., Kjølholt, J., Høibye, L. \& Hinge-Christensen, S., 2005. Survey of Estrogenic Activity in the Danish Aquatic Environment, s.l.: s.n.

Svenson, A. et al., 2009. IVL B1826: Results from the Swedish National Screening Programme 2007 Sub report 5: Silver, s.l.: IVL. 
Thomas, K. et al., 2007. TA-2246: Occurrence of selected pharmaceuticals in wastewater effluents from hospitals (Ullevål and Rikshospitalet) and VEAS wastewater treatment works, s.l.: SFT (Klif).

Törneman, N., 2011b. Screening of N,N-dietyl-m-toluamid (DEET), s.l.: SWECO.

Törneman, 2010. Result Screening 2009 - 2010: Screening of musk substances and metabolites, s.l.: Sweco.

Törneman, N., 2008. Screening report 2008:2: Screening of musk substances, s.l.: Sweco.

Törneman, N., 2011a. Environment Screening Report Chlorhexidine and pchloroaniline, s.l.: Sweco.

Vieno, N., 2008. Occurrence of Pharmaceuticals in Finnish Sewage Treatment Plants, Surface Waters, and Their Elimination in Drinking Water Treatment Processes, s.l.: Tampere University of Technology. Publication 667.

Vogelsang, C., 2008. Renseanlegg / sykehusavløp. [Online] Available at: http://www.tekna.no/ikbViewer/Content/534870/\%286\%29\%20Christian\%20V ogelsang.pdf [Accessed December 2011].

Woldegiorgis, A. et al., 2007b. IVL B1751: Results from the Swedish screening 2006 Sub report 4: Pharmaceuticals, s.l.: IVL.

Woldegiorgis, A. et al., 2007a. B1764: Results from the Swedish screening program 2006 - Subreport 3: Zinc pyrithione and Irgarol 1051, s.l.: IVL. 
Nordic Council of Ministers

Ved Stranden 18

DK-1061 Copenhagen K

www.norden.org

\section{PPCP monitoring in the Nordic Countries}

\section{- Status Report}

The Nordic Screening group is pleased to present a compilation of pharmaceuticals and personal care products (PPCPs) monitoring data from the Nordic countries. The report has been prepared by Bergfald miljørådgivere and founded by the Nordic Council of Ministers via the Aquatic Ecosystems Group and the Nordic Chemicals Group.

In recent years, there has been an increased awareness of environmental effects from our use of PPCPs. In order to examine the occurrence of PPCPs in the environment, the Nordic countries have conducted several regional and national environmental surveys. The Nordic screening group saw the need for a compilation of these studies. The objective of this report was therefore to present the existing Nordic environmental data on PPCPs, by identifying which compounds have been analysed, in which countries and if there are obvious variations of levels detected in each country.

The Nordic screening is run by a steering group with representatives from the Danish Centre for environment and Energy, Aarhus University, the Finnish Environment Institute, the Environment Agency of Iceland, the Environment Agency of the Faroe Islands, the Norwegian Climate and Pollution Agency and the Swedish Environmental Protection Agency. 